\title{
Avian influenza A virus adaptation to the equine host and identification of host-specific markers
}

\author{
V. MUCHA, J. HOLLÝ, E.VAREČKOVÁ, F. KOSTOLANSKÝ*
}

Institute of Virology, Biomedical Research Center Slovak Academy of Sciences, Dúbravská cesta 9, 84505 Bratislava, Slovak Republic

Received December 15, 2017; accepted July 6, 2018

\begin{abstract}
Summary. - Avian influenza A viruses (IAVs) are able to overcome the interspecies barrier and adapt to the new non-avian host. The process of adaptation requires the adaptive changes of IAV genome resulting in amino acid substitutions. The aim of this work was the description of amino acid substitutions in avian influenza A viruses (IAVs) occurring during their adaptation to equine host. Today, viruses of the equine influenza H3N8 subtype, first isolated in 1963, represent a single genetic lineage of IAV causing a respiratory disease in horses. We compared the amino acid sequences of the conserved proteins PB2, PB1, PA, NP, M1, M2, NS1 and NEP of equine influenza H3N8 subtype IAV with sequences of avian viruses, both available in the NCBI's Influenza Virus Resource Database. The amino acid substitutions persisting in equine IAV isolates and occurring in avian IAV at $<5 \%$ frequency were included into the list of equine host-specific markers. We documented amino acid substitutions in the examined IAV proteins of equine IAV isolates in the period 1963-2013 and observed their quasi-linear accumulation. The substitution rate for the virus ribonucleoprotein (RNP) complex (PB2, PB1, PA, and NP) was calculated as $\mathrm{R}=0.69 \times 10^{-3}$ per position per year. For other examined proteins (M1, M2, NS1, and NEP), calculated R-values ranged from $0.48 \times 10^{-3}$ to $1.30 \times 10^{-3}$ per position per year. We identified 127 equine host markers distributed among all internal virus proteins, 38 of which were present already in 1963 and other 89 accumulated during the period since 1963 until 2013. Ten equine host marker positions overlap with known human marker positions (Miotto et al., 2010) and five of them are occupied with identical amino acids in IAV of both hosts.
\end{abstract}

Keywords: equine influenza virus; equine H3N8 virus lineage; adaptation to host; amino acid substitution; host-specific marker

\section{Introduction}

Waterfowl is considered to be a reservoir of influenza A viruses (IAVs), which are potentially able to replicate and spread to new hosts after overcoming the interspecies barrier (Webster et al., 1992). The best-described examples are the influenza pandemic that spread in humans in the $20^{\text {th }}$ century, the repeated transmissions of highly pathogenic avian $\mathrm{H} 5 \mathrm{~N} 1$ viruses (Subbarao and Shaw, 2000), and the

"Corresponding author. E-mail: virufkos@savba.sk; phone: +4212-59302454.

Abbreviations: $\operatorname{IAV}(\mathrm{s})=$ influenza A virus(es); RNP = ribonucleoprotein recently described H7N9 influenza zoonotic infections of humans (Gao et al., 2013). As these zoonotic IAV infections are considered to be a major epidemic threat for humans, the study of the evolutionary process of IAVs and their adaptation to new mammalian hosts is of high importance. Relevant data on amino acid changes accumulated in viral proteins during the adaptation of avian IAVs to humans were collected using a large number of IAV nucleotide genome sequences and amino acid sequences of the corresponding IAV proteins. The analysis of IAV human host markers is of great interest because disclosing the possible adaptation changes occurring over time can help to defend humans against zoonoses with pandemic potential. Sixty-eight human host markers in the PB2, PB1, PA, NP, M1, M2, NS1, NS2 (NEP) proteins were described by Miotto (Miotto et al., 
2010). These markers distinguish human IAV strains from those circulating among birds.

IAVs cause respiratory diseases also in horses. The first equine isolate of IAV was obtained in 1956 in Czechoslovakia and was characterized as an H7N7 subtype (A/eq/Prague/56) (Sovinova et al., 1958). Equine H7N7 represented a distinct line, which disappeared after the year 1977, when the last equine H7N7 virus was isolated. During the outbreak of respiratory disease of horses in Florida in 1963, IAV of the H3N8 subtype (A/equine/Miami/1/1963[H3N8]) was isolated for the first time (Waddell et al., 1963). Since the late 1970s until present, the H3N8 subtype has been the dominant equine IAV and persisted in horses until now (Yoon et al., 2014).

Aquatic birds are considered to be a reservoir not only of human, but also of equine IAV. During bird-to-horse IAV transmission, and subsequent adaptation to equine host, viruses undergo changes in the genome and consequently in the encoded proteins, resulting in the ability to replicate and spread in equine hosts.

Utilizing the NCBI's Influenza Virus Resource database, we analysed IAVs of the H3N8 subtype isolated from equine hosts from the period 1963 up to the present, in order to describe the natural course of avian-to-equine IAV adaptation. We determined conserved equine IAV marker amino acids, which were characterized with respect to the time of their appearance, uniqueness for equine host, incidence in individual proteins, the type of amino acid substitution and the possible influence on the function of a particular protein. A comparison of equine host markers with those described in human IAV was also performed.

\section{Materials and Methods}

Equine IAV H3N8 viruses. Amino acid sequences of the conserved proteins (i.e., PB2, PB1, PA, NP, M1, M2, NS1, and NEP) of all equine influenza A H3N8 isolates (between 1963, when the first isolate was obtained, and 2013) found in the NCBI's Influenza Virus Resource database (Bao et al., 2008) were utilized to perform the alignments.

Alignment of influenza A H3N8 equine lineage proteins. Accession numbers and alignments of aa sequences of analysed proteins of virus isolates with identified equine host markers are given in Supplementary Tables.

The amino acid sequences of a working set of each protein of interest were sorted by the year of isolation. Then, sequence alignment of the individual proteins was executed online at http://www. ncbi.nlm.nih.gov/genomes/FLU/FLU.html.

Substitution rate determination. For each protein, a plot was constructed that showed the accumulation of substitutions in the lineages of individual proteins from 1963 to 2013. The alignment plots were assessed. Outliers, evidently not belonging to the lineage (e.g., the particular sequence was too distant from the H3N8 lineage), were removed. To calculate the intrinsic amino acid substitution rate of influenza A H3N8 equine lineage, a number of substitutions accumulated in a recent (A/equine/Xuzhou/01/2013(H3N8)) in relation to an early A/equine/Miami/1/1963(H3N8)) member of the lineage were counted. This approach was employed due to a quasi-linearity of substitutions that accumulated over time. The substitution rate, i.e., the number of amino acid substitutions per position per year was determined using the formula $R=S /(L \times Y)$, where $S$ is the number of substitutions between two aligned sequences, $\mathrm{L}$ is the length of the protein (number of amino acids), and $\mathrm{Y}$ is time between isolations of the two aligned sequences, measured in years.

For the purpose of calculation of the number of substitutions in NS1 protein, a deleted part of C-terminal amino acids of NS1 protein was taken as a single substitution.

Equine markers identification. To delineate amino acid positions characterizing equine lineages in each internal protein, we compared the sequences of equine lineages with sequences from all avian hosts isolated until 2013, found in the database. This limitation was chosen to reduce the number of isolates, as no more than 10,000 sequences are allowed as input to the Analyze Sequence Variation (SNP) tool at http://www.fludb.org. First, we delineated the accumulation of amino acid substitutions in all internal proteins studied from equine isolates during the period 1963-2013. The year of appearance of any amino acid change that persisted at a given position was determined.

For each amino acid position, the consensus amino acid of the influenza A H3N8 equine lineage together with accumulated substitutions and their frequencies among avian isolates were determined. This data was obtained from the NIAID IRD (Squires $e t$ al., 2012) at http://www.fludb.org. Within the influenza A H3N8 equine lineage we have been looking for amino acid sequence positions occupied with residues considerably distinct from those prevailing in avian isolates that fulfilled the following criteria. We identified amino acid positions that were conserved throughout the whole monitored time-span (1963-2013) and, at the same time, amino acids at these positions differed from those in the avian "consensus" sequence. Several of these amino acid substitutions are fully conserved in equine IAVs, i.e., they have no (or only a few) alternatives since 1963, and they occur among avian sequences at $<5 \%$ frequency. These we refer to as "early" equine-specific markers. Moreover, we identified additional long-lasting conserved substitutions that emerged in years after 1963 and occurred among the avian sequences at $<5 \%$ frequency, which we refer to as "recent" equine-specific markers.

\section{Results}

The sequences of over 100 equine IAVs isolated since 1963 are available in the NCBI's Influenza Virus Resource database. All sequences in the database of equine $\mathrm{H} 3 \mathrm{~N} 8$ isolated between 1963 and 2013 belonged to the genotype 

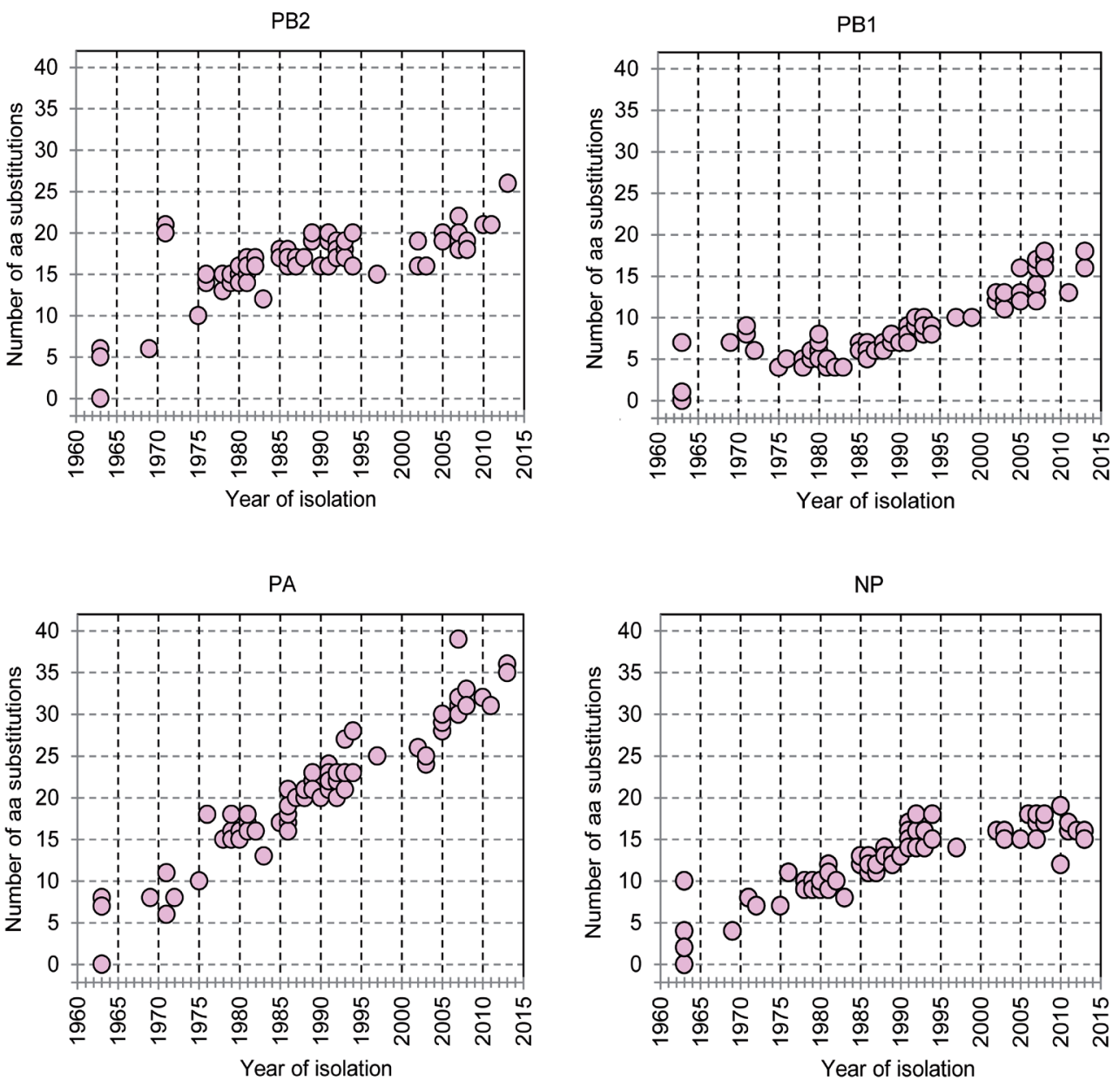

Fig. 1

Accumulation of amino acid substitutions in proteins of the RNP complex of the influenza A H3N8 equine lineage

Each point represents a particular sequence; some sequences are condensed to a single point due to identical coordinates. The number of amino acid substitutions in each isolate was counted with reference to the A/equine/Miami/1/1963 (H3N8) isolate.

comprising individual lineages of virus RNA segments $(\mathrm{C} /$ seg1, I/seg2, G/seg3, 3F/seg4, C/seg5, 8B/seg6, E/seg7, 1D/ seg8) (Lu et al. 2007). Therefore, here we have used the term influenza A H3N8 equine lineage for sequences of this genotype.

Accumulation of substitutions in conserved proteins of the influenza a H3N8 equine lineage

We analysed amino acid sequences of conserved IAV proteins, i.e., proteins of the polymerase complex: $\mathrm{PB} 2, \mathrm{~PB} 1$, $\mathrm{PA}$, and NP; proteins encoded by segment 7 (M1 and M2); as well as by segment 8 (NS1, NEP) of H3N8 equine isolates available in the NCBI's Influenza Virus Resource database (Bao et al., 2008). The amino acid sequences of particular proteins were sorted by the year of isolation. Their alignments showed gradual accumulation of substitutions in proteins over a period of 50 years. Throughout the monitored time-span (1963-2013), the principal influenza A H3N8 equine lineage underwent mutations at several sequence locations, which subsequently became fixed. The plots in Fig. 1 and Fig. 2 demonstrate the number of substitutions within each isolate in comparison with an early virus isolate, A/equine/Miami/1/1963 (H3N8).

The number of substitutions occurring between the first sequenced isolate, A/equine/Miami/1/1963 (H3N8), and the latest isolate A/equine/Xuzhou/01/2013 (H3N8) was determined to calculate the amino acid substitution rate, i.e., the number of substitutions per position per year, for each protein. Among proteins in the polymerase complex, the highest substitution rate was found for PA $(\mathrm{R}=0.98 \times$ $10^{-3}$ amino acid substitutions per position per year), while the lowest rate was found in the PB1 protein with an amino acid substitution rate $\mathrm{R}=0.47 \times 10^{-3}$ (Table 1 ). Substitu- 

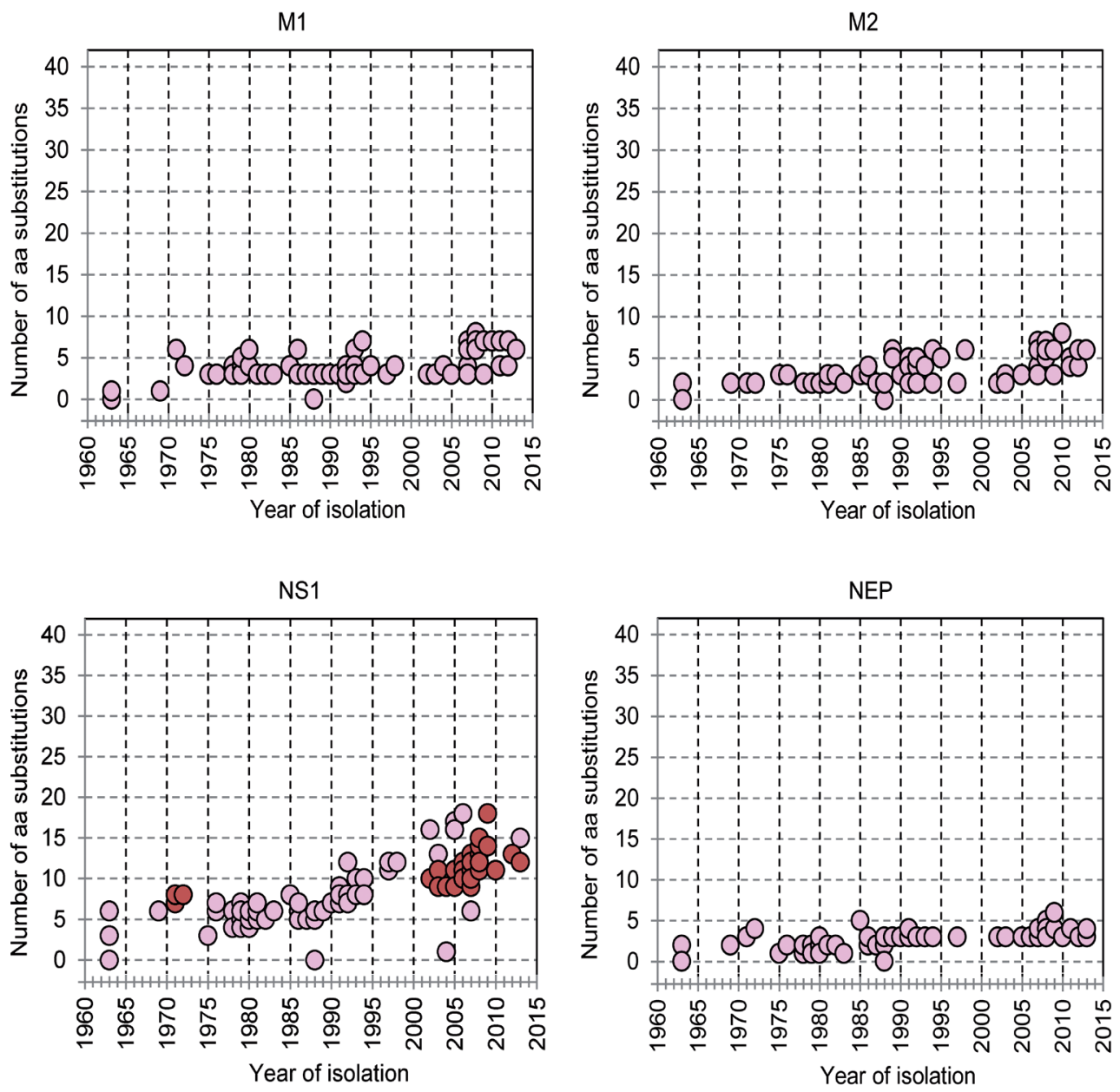

Fig. 2

Accumulation of amino acid substitutions in the M1, M2, NS1, and NEP proteins of the influenza a H3N8 equine lineage Each point represents a particular sequence; some sequences are condensed to a single point due to identical coordinates. The number of amino acid substitutions in each isolate was counted with reference to the A/equine/Miami/1/1963 (H3N8) isolate. Dark-coloured circles (in NS1) stand for sequences in which C-terminal deletions were counted as a single substitution.

tion rates of NP $\left(0.64 \times 10^{-3}\right)$ and PB2 $\left(0.69 \times 10^{-3}\right)$ and the substitution rate for the entire polymerase complex (PB1, $\mathrm{PB} 2, \mathrm{PA}$, and NP), $\mathrm{R}=0.69 \times 10^{-3}$ per position per year, were calculated. The following substitution rates were calculated for proteins encoded by segments 7 and $8: \mathrm{R}=0.48 \times 10^{-3}$ for $\mathrm{M} 1, \mathrm{R}=1.24 \times 10^{-3}$ for $\mathrm{M} 2, \mathrm{R}=1.30 \times 10^{-3}$ for $\mathrm{NS} 1$, and $\mathrm{R}=0.66 \times 10^{-3}$ for NEP (Table 1 ). However, the linearity of amino acid substitution accumulation in proteins coded by segments 7 and 8 was not as apparent as it was in proteins of the RNP complex (compare Fig. 1 and Fig. 2).

\section{Equine host markers}

The amino acid sequences of PB2, PB1, PA, NP, M1, M2, NS1, and NEP of influenza a H3N8 equine lineage were aligned with influenza a sequences isolated from avian hosts, which have been deposited in the NIAID Influenza Research database (IRD) at http://www.fludb.org (Squires et al., 2012). Protein sequence variance analysis provided the "consensus" sequences of IAVs (all subtypes) isolated from avian hosts and a survey of alternative amino acids occurring at individual positions.

Amino acids defined as equine markers, which distinguish equine viruses from avian strains, are summarized in Table 2,3 , and 4 . The frequency of an equine-specific amino acid at a given position among avian IAVs $(<5 \%)$ reflects the equine host marker potency: the lower is the occurrence of an equine-specific amino acid among avian sequences, the higher is its marker potency. In proteins $\mathrm{PB} 2, \mathrm{~PB} 1, \mathrm{PA}$, NP, NS1, and NEP, we found both early and recent equine- 
Table 1. Amino acid substitutions accumulated in proteins of the influenza A H3N8 equine lineage (period 1963-2013)

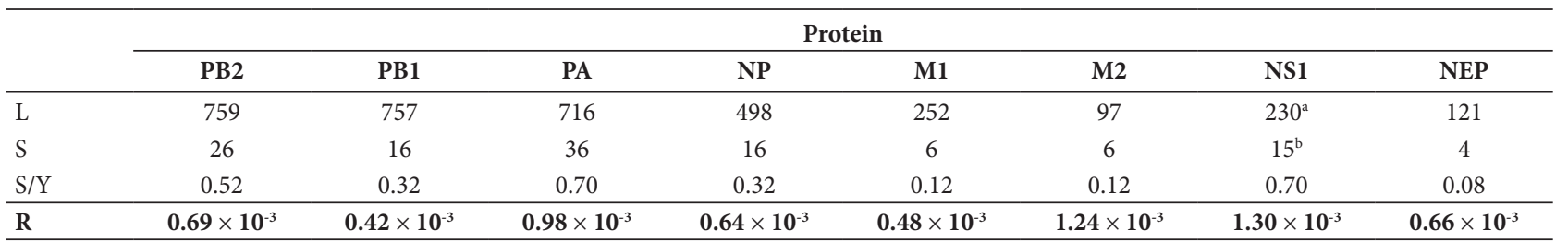

$\mathrm{L}=$ length of the protein (number of amino acids); $\mathrm{S}=$ number of substitutions between 2 aligned sequences, the early isolate A/equine/Miami/1/1963 (H3N8) and the late isolate A/equine/Xuzhou/01/2013 (H3N8); Y = time between the isolations of the 2 aligned sequences (50 years); $\mathrm{R}=$ substitution

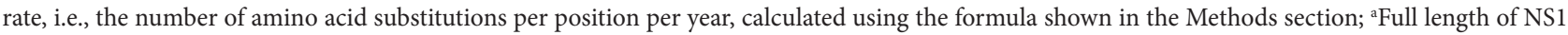
(without deletions in the $\mathrm{C}$-terminus); ${ }^{\mathrm{b}} \mathrm{C}$-terminal deletions occurring in some isolates were counted up as a single substitution.

Table 2. Equine-specific markers identified in the equine influenza H3N8 virus proteins PB2 and PB1

\begin{tabular}{|c|c|c|c|c|}
\hline & \multicolumn{3}{|c|}{ Equine-specific markers in PB2 } & \multirow{2}{*}{$\begin{array}{c}\text { Avian } \\
\text { Consensus }\end{array}$} \\
\hline Pos. & $\begin{array}{c}\text { Marker } \\
(\%)^{\mathrm{a}}\end{array}$ & $\begin{array}{c}\text { Year of isola- } \\
\text { tion }\end{array}$ & $\begin{array}{l}\text { Marker } \\
\text { potency }\end{array}$ & \\
\hline \multicolumn{5}{|c|}{ Early equine markers } \\
\hline 530 & I (100) & 1963 & 0.0005 & $\mathrm{~T}$ \\
\hline 559 & $\begin{array}{l}\text { I (89) } \\
\text { F (55) }\end{array}$ & $\begin{array}{l}1963-2013 \\
1989-1994\end{array}$ & $\begin{array}{l}0.0307 \\
0.0001\end{array}$ & $\mathrm{~T}$ \\
\hline 586 & $\mathbf{R}(100)$ & 1963 & 0.0113 & K \\
\hline 701 & $\mathbf{N}(100)$ & 1963 & 0.0009 & $\mathrm{D}$ \\
\hline \multicolumn{5}{|c|}{ Recent equine markers } \\
\hline 12 & $\mathbf{L}(100)$ & 2002 & 0.0080 & $S$ \\
\hline 105 & $\mathbf{A}(72)$ & 2007 & 0.0334 & $\mathrm{~T}$ \\
\hline 109 & I (98) & 1975 & 0.0463 & $\mathrm{~V}$ \\
\hline 147 & $\mathbf{V}(100)$ & 1976 & 0.0285 & I \\
\hline 251 & $\mathbf{K}(72)$ & 2007 & 0.0150 & $\mathrm{R}$ \\
\hline 299 & K (99) & 1975 & 0.0191 & $\mathrm{R}$ \\
\hline 344 & $\mathbf{M}(98)$ & 1985 & 0.0173 & $\mathrm{~V}$ \\
\hline 511 & I (100) & 1985 & 0.0496 & $\mathrm{~V}$ \\
\hline 575 & I (99) & 1976 & 0.0070 & $\mathrm{M}$ \\
\hline 588 & T (99) & 1975 & 0.0282 & A \\
\hline 613 & $\mathbf{A}(100)$ & 1975 & 0.0109 & $\mathrm{~V}$ \\
\hline 715 & $\mathbf{K}(100)$ & 1975 & 0.0005 & $\mathrm{~N}$ \\
\hline \multirow[t]{2}{*}{717} & $\mathrm{~T}(60)$ & 1971 & 0.0213 & A \\
\hline & $\mathrm{N}=98$ & & & $\begin{array}{c}\mathrm{N}=9580 \\
(2011)\end{array}$ \\
\hline
\end{tabular}

\begin{tabular}{|c|c|c|c|c|}
\hline \multirow[b]{2}{*}{ Pos. } & \multicolumn{3}{|c|}{ Equine-specific markers in PB1 } & \multirow{2}{*}{$\begin{array}{c}\text { Avian } \\
\text { Consensus }\end{array}$} \\
\hline & $\begin{array}{c}\text { Marker } \\
(\%)^{\mathrm{a}}\end{array}$ & $\begin{array}{c}\text { Year of } \\
\text { isolation }\end{array}$ & $\begin{array}{l}\text { Marker } \\
\text { potency }\end{array}$ & \\
\hline \multicolumn{5}{|c|}{ Early equine markers } \\
\hline 157 & $\mathbf{S}(100)$ & 1963 & 0.0020 & A \\
\hline 214 & $\mathbf{R}(97)$ & 1963 & 0.0355 & $\mathrm{~K}$ \\
\hline 261 & $\mathbf{R}(100)$ & 1963 & 0.0000 & S \\
\hline 383 & $\mathbf{D}(100)$ & 1963 & 0.0216 & $\mathrm{E}$ \\
\hline 429 & $\mathbf{R}(100)$ & 1963 & 0.0062 & $\mathrm{~K}$ \\
\hline 642 & $\mathbf{S}(98)$ & 1963 & 0.0355 & $\mathrm{~N}$ \\
\hline \multicolumn{5}{|c|}{ Recent equine markers } \\
\hline 61 & I $(100)$ & 1975 & 0.0006 & $\mathrm{~T}$ \\
\hline 114 & $\mathbf{I}(100)$ & 1997 & 0.0133 & $\mathrm{~V}$ \\
\hline 119 & $\mathbf{M}(76)$ & 2007 & 0.0036 & $\mathrm{~V}$ \\
\hline 164 & $\mathbf{M}(100)$ & 1975 & 0.0025 & I \\
\hline 198 & $\mathbf{R}(100)$ & 1988 & 0.0048 & K \\
\hline 221 & $\mathbf{T}(100)$ & 1997 & 0.0004 & A \\
\hline 317 & $\mathbf{I}(100)$ & 1997 & 0.0254 & M \\
\hline 329 & $\mathbf{R}(76)$ & 2007 & 0.0000 & Q \\
\hline 377 & $\mathbf{E}(76)$ & 2007 & 0.0017 & $\mathrm{D}$ \\
\hline 397 & $\mathbf{V}(100)$ & 1986 & 0.0022 & I \\
\hline 457 & G (71) & $2007-2008$ & 0.0023 & $\mathrm{E}$ \\
\hline 587 & $\mathbf{T}(100)$ & 1975 & 0.0070 & A \\
\hline 618 & $\mathbf{D}(76)$ & 2007 & 0.0102 & $\mathrm{E}$ \\
\hline 621 & $\mathbf{K}(81)$ & $1975-2013$ & 0.0152 & \\
\hline \multirow{3}{*}{738} & $\mathbf{R}(46)$ & $2005-2013$ & 0.0214 & Q \\
\hline & $\mathbf{D}(100)$ & 1997 & 0.0017 & $\mathrm{E}$ \\
\hline & $\mathrm{N}=102$ & & & $\begin{array}{c}\mathrm{N}=9609 \\
(2011)\end{array}$ \\
\hline
\end{tabular}

aPer cent of occurence of a marker amino acid in a group of equine isolates since indicated year up to 2013 or as indicated. Marker potency is printed in bold if an equine marker amino acid occurs among avian influenza sequences at less than $1 \%$ frequency. Consensus sequence of avian IAV refers to the most frequent amino acid at a given position. $\mathrm{N}$ designates the number of analysed sequences, and (yyyy) means that all utilized avian strains were isolated until the indicated year.

specific markers. In contrast, no early markers were identified in the M1 and M2 protein sequences.

We did not include into the list of equine markers (Table 2,3 , and 4) those substitutions, which occurred during the monitored time-span, and reverted to the avian consensus sequence, nor substitutions that occurred after the year 2008, the stability of which is hard to predict.

The number of marker positions per unit length of the protein, i.e. the density of equine marker positions for individual proteins was determined as follows: NS1 (7.4\%), NP (5.0\%), PA (4.8\%), NEP (4.1\%), M2 (4.1\%) PB1 (2.7\%), PB2 (2.2\%), M1 (1.6\%).

Comparison of the influenza A H3N8 equine lineage to avian influenza sequences revealed 127 equine-specific 
Table 3. Equine-specific markers identified in the equine influenza H3N8 virus proteins PA and NP

\begin{tabular}{|c|c|c|c|c|}
\hline \multirow[b]{2}{*}{ Pos. } & \multicolumn{3}{|c|}{ Equine-specific markers in PA } & \multirow{2}{*}{$\begin{array}{c}\text { Avian } \\
\text { Consensus }\end{array}$} \\
\hline & $\begin{array}{c}\text { Marker } \\
(\%)^{\mathrm{a}}\end{array}$ & $\begin{array}{c}\text { Year of } \\
\text { isolation }\end{array}$ & $\begin{array}{l}\text { Marker } \\
\text { potency }\end{array}$ & \\
\hline \multicolumn{5}{|c|}{ Early equine markers } \\
\hline 61 & $\mathbf{V}(98)$ & 1963 & 0.0342 & I \\
\hline 63 & I (99) & 1963 & 0.0338 & $\mathrm{~V}$ \\
\hline 99 & $\mathbf{R}(95)$ & 1963 & 0.0104 & G \\
\hline \multirow{2}{*}{217} & $\mathbf{H}(100)$ & $1963-1972$ & 0.0000 & \multirow{2}{*}{ Q } \\
\hline & $\mathbf{Y}(100)$ & $1975-2013$ & 0.0000 & \\
\hline \multirow{3}{*}{270} & $\mathbf{I}(82)$ & 1963-1994 & 0.0007 & \multirow{3}{*}{$\mathrm{L}$} \\
\hline & $\mathbf{M}(100)$ & $1997-2013$ & 0.0003 & \\
\hline & IM (100) & $1963-2013$ & 0.0010 & \\
\hline 272 & $\mathbf{G}(100)$ & 1963 & 0.0164 & $\mathrm{D}$ \\
\hline 336 & $\mathbf{Q}(100)$ & 1963 & 0.0002 & $\mathrm{~L}$ \\
\hline \multirow[t]{2}{*}{689} & $\mathbf{S}(100)$ & 1963 & 0.0010 & A \\
\hline & \multicolumn{3}{|c|}{ Recent equine markers } & \\
\hline 55 & $\mathbf{N}(100)$ & 1975 & 0.0116 & $\mathrm{D}$ \\
\hline 57 & $\mathbf{L}(100)$ & 1972 & 0.0003 & $\mathrm{R}$ \\
\hline 62 & $\mathbf{V}(100)$ & 1997 & 0.0282 & I \\
\hline 64 & $\mathbf{D}(75)$ & 2007 & 0.0012 & $\mathrm{E}$ \\
\hline 86 & $\mathbf{I}(75)$ & 2007 & 0.0112 & M \\
\hline 100 & A (96) & 1975 & 0.0187 & $\mathrm{~V}$ \\
\hline 114 & $\mathbf{K}(78)$ & 2008 & 0.0035 & $\mathrm{E}$ \\
\hline 118 & V (99) & 1975 & 0.0229 & I \\
\hline 158 & $\mathbf{R}(60)$ & 2007 & 0.0147 & $\mathrm{~K}$ \\
\hline 213 & K (97) & 2002 & 0.0128 & $\mathrm{R}$ \\
\hline 216 & $\mathbf{N}$ (99) & 1976 & 0.0206 & $\mathrm{D}$ \\
\hline 231 & $\mathbf{V}(100)$ & 1987 & 0.0005 & A \\
\hline 237 & $\mathbf{K}(75)$ & 2007 & 0.0206 & $\mathrm{E}$ \\
\hline 244 & $\mathbf{S}(100)$ & 1975 & 0.0002 & G \\
\hline 261 & $\mathbf{S}(100)$ & 1975 & 0.0013 & $\mathrm{~L}$ \\
\hline 277 & H (99) & 1976 & 0.0006 & S \\
\hline 343 & $\mathbf{E}(97)$ & 1997 & 0.0002 & A \\
\hline 345 & I (97) & 1997 & 0.0053 & $\mathrm{~L}$ \\
\hline 353 & $\mathbf{R}(94)$ & 2002 & 0.0175 & $\mathrm{~K}$ \\
\hline 400 & T (99) & 1975 & 0.0092 & PSQ \\
\hline 437 & $\mathbf{Y}(100)$ & 1975 & 0.0180 & $\mathrm{H}$ \\
\hline 450 & I (97) & 1994 & 0.0054 & $\mathrm{~V}$ \\
\hline 476 & $\mathbf{T}(75)$ & 2007 & 0.0008 & A \\
\hline 479 & $\mathbf{E}(100)$ & 1985 & 0.0022 & $\mathrm{D}$ \\
\hline 532 & F (98) & 1987 & 0.0000 & $\mathrm{~L}$ \\
\hline 683 & I (100) & 1975 & 0.0123 & $\mathrm{~L}$ \\
\hline & $\mathrm{N}=100$ & & & $\begin{array}{c}\mathrm{N}=9580 \\
(2011)\end{array}$ \\
\hline
\end{tabular}

\begin{tabular}{|c|c|c|c|c|}
\hline \multirow[b]{2}{*}{ Pos. } & \multicolumn{3}{|c|}{ Equine-specific markers in NP } & \multirow{2}{*}{$\begin{array}{c}\text { Avian } \\
\text { Consensus }\end{array}$} \\
\hline & $\begin{array}{c}\text { Marker } \\
(\%)^{\mathrm{a}}\end{array}$ & $\begin{array}{c}\text { Year of } \\
\text { isolation }\end{array}$ & $\begin{array}{l}\text { Marker } \\
\text { potency }\end{array}$ & \\
\hline \multicolumn{5}{|c|}{ Early equine markers } \\
\hline 41 & $\mathbf{V}(100)$ & 1963 & 0.0250 & I \\
\hline 117 & $\mathbf{M}(99)$ & 1963 & 0.0003 & $\mathrm{R}$ \\
\hline 146 & $\mathbf{T}(100)$ & 1963 & 0.0062 & A \\
\hline 245 & G (98) & 1963 & 0.0021 & $\mathrm{~S}$ \\
\hline 305 & $\mathbf{K}(100)$ & 1963 & 0.0065 & $\mathrm{R}$ \\
\hline 345 & $\mathbf{N}(100)$ & 1963 & 0.0003 & S \\
\hline 351 & $\mathbf{K}(100)$ & 1963 & 0.0451 & $\mathrm{R}$ \\
\hline & $\mathbf{A}(83)$ & $1963-2013$ & 0.0049 & \\
\hline 359 & $\mathrm{~T}^{\mathrm{b}}(54)$ & 2002-2012 & 0.0001 & $\mathrm{~S}$ \\
\hline & $\operatorname{AT}(100)$ & $1963-2013$ & 0.0050 & \\
\hline 374 & I (99) & 1963 & 0.0216 & M \\
\hline 453 & $\mathbf{S}(97)$ & 1963 & 0.0041 & $\mathrm{P}$ \\
\hline 496 & $\mathbf{F}(99)$ & 1963 & 0.0146 & $\mathrm{Y}$ \\
\hline \multicolumn{5}{|c|}{ Recent equine markers } \\
\hline 16 & D (98) & 1988 & 0.0005 & G \\
\hline 50 & $\mathbf{N}(98)$ & 1976 & 0.0192 & S \\
\hline 100 & $\mathbf{K}(98)$ & 1985 & 0.0030 & $\mathrm{R}$ \\
\hline 111 & $\mathbf{H}(99)$ & $1976-2012$ & 0.0018 & $\mathrm{Y}$ \\
\hline 111 & $\mathbf{Q}(100)$ & 2013-2013 & 0.0000 & $Y$ \\
\hline 136 & I (67) & 2007 & 0.0010 & $\mathrm{~L}$ \\
\hline 257 & $\mathbf{T}(67)$ & 2007 & 0.0081 & I \\
\hline 286 & $\mathbf{T}(98)$ & 1988 & 0.0001 & A \\
\hline 293 & $\mathbf{K}(100)$ & 1975 & 0.0113 & $\mathrm{R}$ \\
\hline 312 & I (100) & 1975 & 0.0032 & $\mathrm{~V}$ \\
\hline 319 & $\mathbf{K}(100)$ & 1975 & 0.0038 & $\mathrm{~N}$ \\
\hline 344 & $\mathbf{L}(100)$ & 1985 & 0.0103 & $S$ \\
\hline 384 & K (95) & 1997 & 0.0181 & $\mathrm{R}$ \\
\hline 397 & S (97) & 1975 & 0.0262 & $\mathrm{~N}$ \\
\hline \multirow[t]{2}{*}{498} & $\mathbf{S}(99)$ & 1975 & 0.0157 & $\mathrm{~N}$ \\
\hline & $\mathrm{N}=110$ & & & $\begin{array}{c}\mathrm{N}=9580 \\
(2011)\end{array}$ \\
\hline
\end{tabular}

aPer cent of occurence of a marker amino acid in a group of equine isolates since indicated year up to 2013 or as indicated. ${ }^{b}$ Amino acid T (NP protein) has co-circulated with a since 2002. Marker potency is printed in bold if an equine marker amino acid occurs among avian influenza sequences at less than $1 \%$ frequency. Consensus sequence of avian IAV refers to the most frequent amino acid at a given position. $\mathrm{N}$ designates the number of analysed sequences, and (уyyy) means that all utilized avian strains were isolated until the indicated year.

marker amino acids in the analysed proteins. Of these, 38 early markers persisted since 1963 and additional 89 recent markers continued to emerge after 1963. Among all markers, 69 are of high potency ( 21 early and 48 recent), having $<1 \%$ occurrence among avian influenza amino acid sequences (Table 2, 3, and 4).

\section{Relationship of equine and human host markers}

We compared equine host marker positions described above with those located at 'characteristic sites' identified in human influenza viruses by other authors (Finkelstein et al., 2007; Miotto et al., 2010) (Table 5). While Miotto found 68 human characteristic sites in PB2, PB1, PA, NP, M1, M2, 
Table 4. Equine-specific markers identified in the equine influenza H3N8 virus proteins M1, M2, NS1, and NEP

\begin{tabular}{|c|c|c|c|c|}
\hline & \multicolumn{3}{|c|}{ Equine-specific markers in M1 } & Avian \\
\hline Pos. & $\begin{array}{c}\text { Marker } \\
(\%)^{\mathrm{a}}\end{array}$ & $\begin{array}{c}\text { Year of } \\
\text { isolation }\end{array}$ & $\begin{array}{l}\text { Marker } \\
\text { potency }\end{array}$ & Consensus \\
\hline \multicolumn{5}{|c|}{ Recent equine markers } \\
\hline 80 & I (79) & 2007 & 0.0015 & V \\
\hline 85 & $\mathbf{S}(99)$ & 1975 & 0.0027 & $\mathrm{~N}$ \\
\hline \multirow[t]{2}{*}{95} & $\mathbf{K}(79)$ & 2007 & 0.0243 & $\mathrm{R}$ \\
\hline & $\mathbf{K}(86)$ & $1976-2013$ & 0.0000 & \\
\hline \multirow[t]{3}{*}{208} & R (99) & $2003-2013$ & 0.0003 & Q \\
\hline & KR (99) & $1976-2013$ & 0.0003 & \\
\hline & No. $=146$ & & & $\begin{array}{c}N=9938 \\
(2010)\end{array}$ \\
\hline
\end{tabular}

\begin{tabular}{|c|c|c|c|c|}
\hline \multirow[b]{2}{*}{ Pos. } & \multicolumn{3}{|c|}{ Equine-specific markers in M2 } & \multirow{2}{*}{$\begin{array}{c}\text { Avian } \\
\text { Consensus }\end{array}$} \\
\hline & $\begin{array}{c}\text { Marker } \\
(\%)^{\mathrm{a}}\end{array}$ & $\begin{array}{c}\text { Year of } \\
\text { isolation }\end{array}$ & $\begin{array}{l}\text { Marker } \\
\text { potency }\end{array}$ & \\
\hline \multicolumn{5}{|c|}{ Recent equine markers } \\
\hline 48 & $\mathbf{S}(72)$ & 2007 & 0.0017 & $\mathrm{~F}$ \\
\hline 50 & F (99) & 1975 & 0.0057 & $\mathrm{C}$ \\
\hline 85 & $\mathbf{S}(78)$ & 2007 & 0.0000 & $\mathrm{D}$ \\
\hline 89 & $\mathbf{S}(76)$ & 2007 & 0.0217 & G \\
\hline & $\mathrm{N}=144$ & & & $\begin{array}{c}\mathrm{N}=9571 \\
(2010)\end{array}$ \\
\hline
\end{tabular}

\begin{tabular}{|c|c|c|c|c|}
\hline & \multicolumn{3}{|c|}{ Equine-specific markers in NS1 } & \multirow{2}{*}{$\begin{array}{c}\text { Avian } \\
\text { Consensus }\end{array}$} \\
\hline Pos. & $\begin{array}{c}\text { Marker } \\
(\%)^{\mathrm{a}}\end{array}$ & $\begin{array}{c}\text { Year of } \\
\text { isolation }\end{array}$ & $\begin{array}{l}\text { Marker } \\
\text { potency }\end{array}$ & \\
\hline \multicolumn{5}{|c|}{ Early equine markers } \\
\hline 96 & D (96) & 1963 & 0.0007 & $\mathrm{E}$ \\
\hline 139 & E (99) & 1963 & 0.0044 & $\mathrm{D}$ \\
\hline \multirow[t]{2}{*}{156} & V (98) & 1963 & 0.0032 & I \\
\hline & $\mathbf{H}(90)$ & $1963-2013$ & 0.0006 & \\
\hline \multirow[t]{2}{*}{207} & $\mathbf{Y}(29)$ & 2006-2007 & 0.0000 & $\mathrm{~N}$ \\
\hline & HY (96) & $1963-2013$ & 0.0006 & \\
\hline 209 & $\mathbf{N}(98)$ & 1963 & 0.0326 & $\mathrm{D}$ \\
\hline 213 & $S(96)$ & 1963 & 0.0345 & $\mathrm{P}$ \\
\hline 214 & $\mathbf{F}(100)$ & 1963 & 0.0090 & $\mathrm{~L}$ \\
\hline \multicolumn{5}{|c|}{ Recent equine markers } \\
\hline 45 & $\mathbf{R}(70)$ & $2008-2009$ & 0.0005 & G \\
\hline 48 & I (87) & 2007 & 0.0068 & S \\
\hline 59 & $\mathbf{H}(88)$ & 1997 & 0.0217 & $\mathrm{R}$ \\
\hline 67 & $\mathbf{Q}(97)$ & 1981 & 0.0087 & $\mathrm{R}$ \\
\hline 71 & $\mathbf{K}(90)$ & 1997 & 0.0116 & $\mathrm{E}$ \\
\hline 84 & I (84) & 2006 & 0.0120 & $\mathrm{~V}$ \\
\hline 86 & $\mathbf{T}(88)$ & 1997 & 0.0470 & A \\
\hline 186 & K (98) & 1975 & 0.0059 & $\mathrm{E}$ \\
\hline 194 & I (82) & 1990 & 0.0150 & $\mathrm{~V}$ \\
\hline \multirow[t]{2}{*}{216} & $\mathbf{S}(88)$ & 1997 & 0.0123 & $\mathrm{P}$ \\
\hline & $\mathrm{N}=169$ & & & $\begin{array}{c}\mathrm{N}=9621 \\
(2009)\end{array}$ \\
\hline
\end{tabular}

\begin{tabular}{|c|c|c|c|c|}
\hline \multirow[b]{2}{*}{ Pos. } & \multicolumn{3}{|c|}{ Equine-specific markers in NEP } & \multirow{2}{*}{$\begin{array}{c}\text { Avian } \\
\text { Consensus }\end{array}$} \\
\hline & $\begin{array}{c}\text { Marker } \\
(\%)^{\mathrm{a}}\end{array}$ & $\begin{array}{c}\text { Year of } \\
\text { isolation }\end{array}$ & $\begin{array}{l}\text { Marker } \\
\text { potency }\end{array}$ & \\
\hline \multicolumn{5}{|c|}{ Early equine markers } \\
\hline 35 & $\mathbf{L}(100)$ & 1963 & 0.0160 & $\mathrm{~F}$ \\
\hline 63 & E (99) & 1963 & 0.0102 & G \\
\hline \multicolumn{5}{|c|}{ Recent equine markers } \\
\hline 33 & I (95) & 1976 & 0.0071 & $\mathrm{~T}$ \\
\hline 34 & $\mathbf{R}(96)$ & 1988 & 0.0283 & Q \\
\hline \multirow[t]{2}{*}{89} & $\mathbf{N}(99)$ & 1975 & 0.0083 & I \\
\hline & $\mathrm{N}=130$ & & & $\begin{array}{c}\mathrm{N}=9010 \\
(2009)\end{array}$ \\
\hline
\end{tabular}

aPer cent of occurence of a marker amino acid in a group of equine isolates since indicated year up to 2013 or as indicated. Marker potency is printed in bold if an equine marker amino acid occurs among avian influenza sequences at less than $1 \%$ frequency. Consensus sequence of avian IAV refers to the most frequent amino acid at a given position. $\mathrm{N}$ designates the number of analysed sequences, and (yyyy) means that all utilized avian strains were isolated until the indicated year.

Table 5. Number of marker positions of equine and human influenza viruses

\begin{tabular}{lccc}
\hline \multirow{2}{*}{ Protein } & \multicolumn{2}{c}{ Number of equine markers } & Number of \\
\cline { 2 - 3 } & $\begin{array}{c}\text { Early, } \\
\text { already since } \mathbf{1 9 6 3}\end{array}$ & $\begin{array}{c}\text { All, } \\
\text { fixed until 2013 }\end{array}$ & \\
\hline PB2 & 4 & 17 & 17 \\
PB1 & 6 & 21 & 1 \\
PA & 8 & 34 & 17 \\
NP & 11 & 25 & 12 \\
M1 & 0 & 4 & 3 \\
M2 & 0 & 4 & 9 \\
NS1 & 7 & 17 & 6 \\
NEP & 2 & 5 & 3 \\
\hline All & $\mathbf{3 8}$ & $\mathbf{1 2 7}$ & $\mathbf{6 8}$ \\
\hline
\end{tabular}

${ }^{a}$ Human markers refer to sites characteristic of human-to-human transmission, as reported by Miotto et al. (2010). 
Table 6. Marker positions common for equine and human viruses

\begin{tabular}{lccc}
\hline $\begin{array}{l}\text { Protein/ } \\
\text { position }\end{array}$ & Equine marker & a Human marker & $\begin{array}{c}\text { Prevailing amino } \\
\text { acid in avian host }\end{array}$ \\
\hline $\mathrm{PB} 2 / 588$ & $\mathrm{~T}(1975)$ & $\mathrm{I}$ & $\mathrm{A}$ \\
$\mathrm{PA} / 55^{\mathrm{d}}$ & $\mathrm{N}(1975)$ & $\mathrm{N}$ & $\mathrm{D}$ \\
$\mathrm{PA} / 57$ & $\mathrm{~L}(1972)^{\mathrm{b}}$ & $\mathrm{Q}$ & $\mathrm{R}$ \\
$\mathrm{PA} / 100^{\mathrm{d}}$ & $\mathrm{A}(1975)$ & $\mathrm{A}$ & $\mathrm{V}$ \\
$\mathrm{PA} / 400$ & $\mathrm{~T}(1975)^{\mathrm{b}}$ & $\mathrm{L}$ & $\mathrm{PSQ}$ \\
$\mathrm{NP} / 16^{\mathrm{d}}$ & $\mathrm{D}(1988)^{\mathrm{b}}$ & $\mathrm{D}$ & $\mathrm{G}$ \\
$\mathrm{NP} / 100$ & $\mathrm{~K}(1985)^{\mathrm{b}}$ & $\mathrm{VI}$ & $\mathrm{R}$ \\
$\mathrm{NP} / 136^{\mathrm{d}}$ & $\mathrm{IM}^{\mathrm{c}}$ & $\mathrm{MI}$ & $\mathrm{L}$ \\
$\mathrm{NP} / 305^{\mathrm{d}}$ & $\mathrm{K}(1963)^{\mathrm{b}}$ & $\mathrm{K}$ & $\mathrm{R}$ \\
$\mathrm{NS} 1 / 84$ & $\mathrm{I}(2006)$ & $\mathrm{TA}$ & $\mathrm{VS}$ \\
\hline
\end{tabular}

a Sites characteristic of human-to-human transmission, as reported by Miotto et al. (2010). ${ }^{\mathrm{b}}$ Amino acids occurring in the avian IAV sequences at $\mathrm{a}<1 \%$ frequency, i.e., markers of high potency. ${ }^{\mathrm{c}} \mathrm{Amino}$ acid $\mathrm{M}$ at position $\mathrm{NP} / 136$ occurrs in the avian IAV sequences at a $8.4 \%$ frequency. ${ }^{\mathrm{d}}$ Marker position where the same amino acid both in human and equine virus strains are present.

\section{Discussion}

Aquatic birds are considered to be a reservoir of IAVs, which can overcome interspecies barriers under suitable conditions and infect mammals, including humans. Interspecies transmission of avian viruses requires adaptation changes in the genome, characteristic for a particular new host, to enable optimal replication. The accumulation of adaptive changes in the genome of IAV establishes a new virus lineage associated with the new host. Currently, the best studied human influenza virus lineages are the H1N1 (1918), H2N2 (1957), H3N2 (1968), and H1N1 (2009) subtypes. Less studied are the non-avian IAV lineages of other animal hosts.

During the last 50 years, the sequences of over 100 equine IAV isolates have been determined and are available in public databases. In this study, we analysed sequences of the influenza a H3N8 equine lineage, the only equine lineage currently in circulation. We focused on the conserved proteins, i.e. proteins of the RNA polymerase complex (PB2, PB1, PA, and NP), as well as the M1, M2, NS1, and NEP proteins. The aim was to describe the evolutionary dynamics of these proteins and to identify equine host markers, i.e. their positions and the amino acids characteristic for the equine host, absent or occurring very rarely $(<5 \%)$ among avian influenza sequences. Such "persistent host markers" were described for avian-to-human IAV adaptation (Finkelstein et al., 2007; Miotto et al., 2010).

Our analysis showed that some of equine host markers were already present in 1963, when influenza A H3N8 virus was isolated from horses for the first time. IAV of subtype H7N7 circulated in horses before 1963 and was first isolated in 1956. This lineage was of a different genotype and was competitively eliminated in 1970 's by the onset of the H3N8 lineage (Murcia et al. 2011). The presence of several equine host markers in equine $\mathrm{H} 3 \mathrm{~N} 8$ viruses identified in isolates from 1963 indicates that equine IAVs branched off earlier.

According to our findings, the substitution rate $\mathrm{R}$ for all proteins of the polymerase complex (PB2, PB1, PA, and NP) of equine influenza virus equals $0.69 \times 10^{-3}$ substitutions per year per amino acid position. Our observations did not show any reassortment event in the equine $\mathrm{H} 3 \mathrm{~N} 8$ lineage, as continuity of accumulation of amino acid substitutions was not interrupted since 1963 in any of examined internal genes. We showed that the accumulation of amino acid substitutions over time was linear, as was also shown for the human H1N1 lineage (Carter and Sanford, 2012). Comparison of markers defined here for equine $\mathrm{H} 3 \mathrm{~N} 8$ isolates with those of human $\mathrm{H}(1,2,3) \mathrm{N}(1,2)$ isolates showed that the equine and human lineages are evolutionarily distinct. In the equine H3N8 lineage, similar to human viruses, short-lived side branches were present for a certain period of time and then disappeared, and only one main lineage continues. Our results based on the sequence alignment show that differences between human and equine lineages have continued to grow over time. Accumulation of new equine markers has taken place mainly at positions where human markers are not present, namely at 117 positions out of a total of 127 positions.

Some amino acid substitutions described here as equine host markers can have an impact on the RNA-polymerase activity of the virus, progressively reducing its restrictions in the new host.

In the H3N8 equine lineage, we found a few amino acid substitutions occurring in the region of the PB1-PA interface. An amino acid substitution in PB1 (T61I) emerged in the Influenza A H3N8 equine lineage in 1975. This position is part of the region involved in the PB1 interaction with the PA-linker (aa 196-257) (Pflug et al., 2014). In particular, at PA position 217, histidine was present already in 1963 but was substituted with tyrosine (H217Y) in 1975, and both amino acids have remained exclusively characteristic of equine viruses to date. Position 217 is localized inside a cluster of equine-specific markers $(213,216,217,231,237$, and 244) in the nuclear localisation signal 2 region of PA (aa 186-247) (Nieto et al., 1994; Hu and Liu, 2015).

The E627K substitution in the PB2 protein is a wellknown adaptive change of IAV in mammalian hosts. Ninety-seven per cent of avian isolates have a glutamic acid (E) residue at this position. This substitution overcomes mammalian antiviral RIG-I impairment of vRNPs (Weber et al., 2015). Surprisingly, all equine isolates also exclusively show an E (identically with avian IAVs) at this position. In contrast to avian IAVs, equine isolates possess an additional substitution - D701N. This mutation plays an important role in viral adaptation to the nuclear import machinery 
and enhances the nuclear import of newly synthesized PB2 (Gabriel et al., 2008). The N701 equine marker in PB2 was present already in 1963. Residue N701 is involved in binding mouse host protein importin a5 (Naffakh et al., 2008) and was also described in human $\mathrm{H} 5$ and $\mathrm{H} 7$ isolates (Gabriel and Fodor, 2014). However, it was not found in any human $\mathrm{H} 1 \mathrm{~N} 1$ or $\mathrm{H} 3 \mathrm{~N} 2$ lineages. Other equine-characteristic substitutions N715K and A717T had occurred already in 1975 and 1971, respectively, and these positions, similar to position 701, are localized in the binding region for the host protein importin alpha 5 (Naffakh et al., 2008). The equine-characteristic amino acids at positions 701 and 715 mentioned above are present only in $<0.1 \%$ of avian isolates; therefore, we consider them to be of high importance for IAV replication in equine host. $\mathrm{PB} 2$ protein plays also an important role in recognizing host mRNA caps, essential during IAV replication. Changes in the cap-binding domain of PB2 can influence host specificity. In equine IAV isolates, we found a V344M substitution, localized in the cap-binding domain of PB2 (residues 318-483) described by Guilligay et al. (2008) and by Pflug et al. (2014).

The region restricted by amino acid positions 107-134, the putative active endonuclease site of PA (Yuan et al. 2009), has involved equine host markers K114 and V118, which have been present in equine isolates since 2008 and 1975, respectively.

The NP N319K substitution described here as an equine host marker was shown to be associated with enhanced binding of the NP protein to importin alpha 1 in mammalian cells (Gabriel et al., 2008). We identified an early equine marker V41 on NP. This position was described in the context of enhanced H7N9 IAV replication at lower temperature as potentially contributing to viral transmissibility (Zhu et al., 2015).

NP of IAV plays a crucial role in the resistance to interferon-inducible Mx protein, an antiviral host factor. Similar to human $\mathrm{MxA}$, equine $\mathrm{Mx} 1$ is phylogenetically distant from the avian Mx protein (Haller et al., 2015). Therefore, equine IAVs are also under constant evolutionary pressure by $\mathrm{Mx} 1$ restriction. Amino acid substitutions in NP associated with MxA resistance among human IAVs can be only partially found in the equine NP. The human MxA-resistance NP amino acid cluster is thought to be determined mainly by $\mathrm{V} /$ I100, P283, and Y313 with additional amino acids associated with MxA resistance: N52, D53, H289, Y/V313, K305, M316, K350, K351, I353, K357 (Mänz et al., 2013; Riegger et al. 2015; Götz et al., 2016). Importantly, it is believed that MxAresistance amino acid substitutions must be accompanied by compensatory stabilizing mutations to maintain viral fitness. Such compensatory mutations were determined to occur at amino acids D16, C385, and Y101 (Götz et al., 2016).

We found only three equine-specific amino acids at positions associated with MxA resistance matching the equine NP sequence: K305, K351, and I353. We identified two of them (K305 and K351) as early equine-adaptation markers. The third substitution, V353I, occurs at $>5 \%$ frequency among avian isolates, therefore, we did not include it into the list of equine-specific substitutions. The R100K substitution is a recent marker that may also play a role in overcoming $\mathrm{Mx} 1$ restriction in further virus-adaptation processes. Moreover, we identified a recent equine-adaptation marker (G16D substitution), which is still the only documented compensatory stabilizing substitution that does not abrogate Mx1 resistance acquired by substitutions mentioned above (Götz et al., 2016). It is possible that some early equine markers on NP could represent further stabilizing mutations, like G16D, contributing to viral fitness. There is also an indication that the Mx1 polymorphism may be associated with equine resistance to IAVs (Manuja et al., 2014).

Until the 1970's, influenza M proteins were not involved in the adaptation process to a new equine host, in contrast to other virus proteins. All equine host markers in the M1 and M2 proteins that can influence the functional properties of M1/M2 were introduced in the 1970s or later, which means that in these proteins are present only recent markers. The first adaptation changes in the M1 protein within the influenza a equine H3N8 lineage occurred in 1975 (N85S) and 1976 (Q208K). Recent equine markers identified in the M1 protein (80I, 85S, and 95K) are localized in the amino acid region 76-115, which is essential for NEP binding (Shimizu et al., 2011). In the M2 protein the substitutions C50F and G89S may influence phosphorylation (Holsinger et al., 1995; Schnell et al., 2008), but do not influence M2 ion channel activity. Therefore, changes at these positions are probably not essential for avian IAV adaptation to equine host.

The NS1 protein is composed of two main parts - the effector domain and the RNA-binding domain. These domains comprise numerous sites mediating the various activities of the NS1 protein (Marc, 2014). The equine marker positions in NS1 are almost all localized outside of the known active sites. The only exception is the substitution E96D, which is positioned in the a 4 helix of the effector domain, the binding site for phosphatidylinositol 3-kinase. Amino acid E96 is highly conserved in birds, bats, and human isolates, but the equine H3N8 lineage possesses aspartic acid (D) in this position, which was already present in 1963 isolates. Another amino acid substitution (E186K occurring in 1975) is localized in NS1 contact area with a cellular $30-\mathrm{kDa}$ subunit of cleavage and polyadenylation specificity factor CPSF30 (Das et al., 2008). Both equine markers in NS1 - 96D and $186 \mathrm{~K}$ - are of high potency. The mutation G45R in NS1 was described to induce increased virulence and an earlier robust pro-inflammatory response in mice (Kaewborisuth et al., 2017). The same mutation appeared as a high-potency marker in equine strains in 2008-2009.

Shortened form of NS1 protein, due to the deletion of eleven amino acids at C-terminus, appeared in 2002 in the 
majority of equine lineage isolates. Such shortened form of this protein occurs in avian isolates permanently with a $10 \%$ frequency.

In NEP, the equine marker I89N (emerged in 1975) forms part of the interaction site with M1 and functions in the nuclear export of progeny vRNPs (Shimizu et al., 2011). The nuclear export signals, NES1 and NES2, in NEP are recognized by chromosome region maintenance protein 1 , which mediates export of the viral RNP complex out of the nucleus. The T33I, Q34R, and F35L substitutions, determined as equine-adaptation changes, reside inside NES2 (31-IITQFESLKI-40) and can affect the export of RNP proteins and, consequently, the budding of progeny virions (Li et al., 2015).

\section{Conclusion}

In conclusion, we identified 127 equine host markers that distinguish present-day equine H3N8 viruses from avian IAVs. Thirty-eight of them were already present in 1963 and another 89 accumulated during the period of 1963-2013. Among all 127 equine markers, 69 (21 early and 48 recent) are of high-potency, i.e., the particular amino acid at the given position occurs among avian strains at $<1 \%$ frequency, or they do not occur at all.

We identified 10 overlapping human and equine marker positions in proteins $\mathrm{PB} 2, \mathrm{PA}, \mathrm{NP}$, and NS1, 5 of which represent identical amino acid residues in corresponding positions. This indicates that some common (mammalian?) features arose during the process of adaptation of avian IAV to human or equine host. However, as follows from the comparative analysis of human and equine markers, the great majority of them localize to distinct positions, in a strictly host-specific manner. Such a divergence of markers indicates the breadth of the evolutionary diversity of equine and human IAVs.

Acknowledgments. This work was supported by grants VEGA 2/0146/15 and VEGA 2/106/17 from the Scientific Grant Agency of the Ministry of Education of Slovak Republic and the Slovak Academy of Sciences and by grant APVV-0250-10 from the Slovak Research and Development Agency.

Supplementary information is available in the online version of the paper and in the EXCELL format from corresponding author.

\section{References}

Bao Y, Bolotov P, Dernovoy D, Kiryutin B, Zaslavsky L, Tatusova T, Ostell J, Lipman D (2008): The influenza virus resource at the National Center for Biotechnology Information. J. Virol. 82, 596-601. https://doi.org/10.1128/JVI.02005-07
Carter RW, Sanford JC (2012): A new look at an old virus: patterns of mutation accumulation in the human H1N1 influenza virus since 1918. Theor. Biol. Med. Model. 9, 42. https:// doi.org/10.1186/1742-4682-9-42

Das K, Ma LC, Xiao R, Radvansky B, Aramini J, Zhao L, Marklund J, Kuo RL, Twu KY et al. (2008): Structural basis for suppression of a host antiviral response by influenza a virus. PNAS 105, 13093-13098. https://doi.org/10.1073/ pnas.0805213105

Finkelstein DB, Mukatira S, Mehta PK, Obenauer JC, Su X, Webster RG, Naeve CW (2007): Persistent host markers in pandemic and H5N1 influenza viruses. J. Virol. 81, 10292-10299. https://doi.org/10.1128/JVI.00921-07

Gabriel G, Fodor E (2014): Molecular determinants of pathogenicity in the polymerase complex. Curr. Top. Microbiol. Immunol. 385, 35-60. https://doi.org/10.1007/82 2014 386

Gabriel G, Herwig A, Klenk H-D (2008): Interaction of polymerase subunit PB2 and NP with importin a1 is a determinant of host range of influenza a virus. PLoS Pathog. 4, e1-e11. https://doi.org/10.1371/journal.ppat.0040011

Gao R, Cao B, Hu Y, Feng Z, Wang D et al. (2013): Human infection with a novel avian-origin influenza A (H7N9) virus. N. Engl. J. Med. 368, 1888-1897. https://doi.org/10.1056/ NEJMoa1304459

Götz V, Magar L, Dornfeld D, Giese S, Pohlmann A, Höper D, Kong BW, Jans DA, Beer M et al. (2016): Influenza A viruses escape from MxA restriction at the expense of efficient nuclear vRNP import. Sci. Rep. 6, 23138. https://doi. org/10.1038/srep23138

Guilligay D, Tarendeau F, Resa-Infante P, Coloma R, Crepin T, Sehr P, Lewis J, Ruigrok RWH, Ortin J, Hart DJ, Cusack S (2008): The structural basis for cap binding by influenza virus polymerase subunit PB2. Nat. Struct. Mol. Biol. 15, 500-506. https://doi.org/10.1038/nsmb.1421

Haller O, Staeheli P, Schwemmle M, Kochs G (2015): Mx GTPases: dynamin-like antiviral machines of innate immunity. Trends Microbiol. 23, 154-163. https://doi.org/10.1016/j. tim.2014.12.003

Holsinger LJ, Shaughnessy MA, Micko A, Pinto LH, Lamb RA (1995): Analysis of the posttranslational modifications of the Influenza virus M2 protein. J. Virol. 69, 1219-1225.

Hu J, Liu X (2015): Crucial role of PA in virus life cycle and host adaptation of influenza A virus. Med. Microbiol. Immunol. 204, 137-149. https://doi.org/10.1007/s00430014-0349-y

Kaewborisuth C, Kaplan B, Zanin M, Finkelstein D, Webby RJ, Lekcharoensuk P (2017): G45R on nonstructural protein 1 of influenza A virus contributes to virulence by increasing the expression of proinflammatory cytokines in mice. Arch. Virol. 162, 45-55. https://doi.org/10.1007/ s00705-016-3072-8

Li J, Yu M, Zheng W, Liu W (2015): Nucleocytoplasmic Shuttling of Influenza A virus Proteins. Viruses 7, 2668-2682. https:// doi.org/10.3390/v7052668

Lu G, Rowley T, Garten R, Donis RO (2007): FluGenome: a web tool for genotyping influenza A virus. Nucleic Acids Res. 35, Web Server issue W275-W279. https://doi.org/10.1093/ nar/gkm365 
Manuja BK, Manuja A, Dahiya R, Singh S, Sharma RC, Gahlot SK (2014): Diversity of interferon inducible Mx gene in horses and association of variations with susceptibility vis-à-vis resistance against equine influenza infection. Infect. Genet. Evol. 27, 142-148. https://doi.org/10.1016/j. meegid.2014.07.018

Mänz B, Dornfeld D, Götz V, Zell R, Zimmermann P, Haller O, Kochs G, Schwemmle M (2013): Pandemic Influenza A Viruses Escape from Restriction by Human MxA through Adaptive Mutations in the Nucleoprotein. PLoS Pathog. 9, e1003279. https://doi.org/10.1371/journal.ppat.1003279

Marc D (2014): Influenza virus non-structural protein NS1: interferon antagonism and beyond. J. Gen. Virol. 95, 2594-2611. https://doi.org/10.1099/vir.0.069542-0

Miotto O, Heiny AT, Albrecht R, García-Sastre A, Tan TW, August JT, Brusic V (2010): Complete-proteome mapping of human influenza A adaptive mutations: implications for human transmissibility of zoonotic strains. PLoS ONE 5, e9025. https://doi.org/10.1371/journal.pone.0009025

Murcia PR, Wood JL, Holmes EC (2011): Genome-scale evolution and phylodynamics of equine $\mathrm{H} 3 \mathrm{~N} 8$ influenza A virus. J. Virol. 85, 5312-5322. https://doi.org/10.1128/ JVI.02619-10

Naffakh N, Tomoiu A, Rameix-Welti MA, van der Werf S (2008): Host restriction of avian influenza viruses at the level of the ribonucleoproteins. Annu. Rev. Microbiol. 62, 403-424. https://doi.org/10.1146/annurev. micro.62.081307.162746

Nieto A, de la Luna S, Bfircena J, Portela A, Ortin J (1994): Complex structure of the nuclear translocation signal of influenza virus polymerase PA subunit. J. Gen. Virol. 75, 29-36. https://doi.org/10.1099/0022-1317-75-1-29

Pflug A, Guilligay D, Reich S, Cusack S (2014): Structure of influenza A polymerase bound to the viral RNA promoter. Nature 516, 355-360. https://doi.org/10.1038/nature14008

Riegger D, Hai R, Dornfeld D, Mänz B, Leyva-Grado V, SánchezAparicio MT, Albrecht RA, Palese P, Haller O et al. (2015): The nucleoprotein of newly emerged H7N9 influenza A virus harbors a unique motif conferring resistance to antiviral human MxA. J. Virol. 89, 2241-2252. https:// doi.org/10.1128/JVI.02406-14

Schnell JR, Chou JJ (2008): Structure and mechanism of the M2 proton channel of influenza A virus. Nature 451, 591-595. https://doi.org/10.1038/nature06531
Shimizu T, Takizawa N, Watanabe K, Nagata K, Kobayashi N (2011): Crucial role of the influenza virus NS2 (NEP) $\mathrm{C}$-terminal domain in $\mathrm{M} 1$ binding and nuclear export of vRNP. FEBS Lett. 585, 41-46. https://doi.org/10.1016/j. febslet.2010.11.017

Sovinova O, Tumova B, Pouska F, Nemec J (1958): Isolation of a virus causing respiratory disease in horses. Acta Virol. $2,52-61$.

Squires RB, Noronha J, Hunt V, García-Sastre A, Macken C, Baumgarth N, Suarez D, Pickett BE, Zhang Y et al. (2012): Influenza Research Database: an integrated bioinformatics resource for influenza research and surveillance. Influenza Other Respir. Viruses 6, 404-416. https://doi. org/10.1111/j.1750-2659.2011.00331.x

Subbarao K, Shaw MN (2000): Molecular aspects of avian influenza (H5N1) viruses isolated from humans. Rev. Med. Virol. 10, 337-348. https://doi.org/10.1002/1099-1654(200009/10)10:5<337::AID-RMV292>3.0.CO;2-V

Waddell GH, Teigland MB, Sigel MM (1963): a new influenza virus associated with equine respiratory disease. J. Am. Vet. Med. Assoc. 143, 587-590.

Weber M, Sediri H, Felgenhauer U, Binzen I, Bänfer S, Jacob R, Brunotte L, García-Sastre A, Schmid-Burgk JL et al. (2015): Influenza virus adaptation PB2-627K modulates nucleocapsid inhibition by the pathogen sensor RIG-I. Cell Host Microbe 17, 309-319. https://doi.org/10.1016/j. chom.2015.01.005

Webster RG, Bean WJ, Gorman OT, Chambers TM, Kawaoka Y (1992): Evolution and ecology of influenza A viruses. Microbiol. Rev. 56, 152, 179.

Yoon SW, Webby RJ, Webster RG (2014): Evolution and Ecology of Influenza A Viruses. In Compans RW, Oldstone MBA (Eds): Influenza Pathogenesis and Control - Volume I, Springer International Publishing Switzerland, pp. 359-375. https://doi.org/10.1007/82 2014 396

Yuan P, Bartlam M, Lou Z, Chen S, Zhou J, He X, Lv Z, Ge R, Li $X$ et al. (2009): Crystal structure of an avian influenza polymerase $\mathrm{PA}(\mathrm{N})$ reveals an endonuclease active site. $\mathrm{Na}$ ture 458, 909-913. https://doi.org/10.1038/nature07720

Zhu W, Zou X, Zhou J, Tang J, Shu Y (2015): Residues 41V and/ or 210D in the NP protein enhance polymerase activities and potential replication of novel influenza (H7N9) viruses at low temperature. Virol. J. 12, 71-75. https://doi. org/10.1186/s12985-015-0304-6 


\title{
Supplementary information
}

\section{Avian influenza A virus adaptation to the equine host and identification of host-specific markers}

\author{
V. MUCHA, J. HOLLÝ, E.VAREČKOVÁ, F. KOSTOLANSKÝ
}

Institute of Virology, Biomedical Research Center Slovak Academy of Sciences, Dúbravská cesta 9, 84505 Bratislava, Slovak Republic

Received December 15, 2017; accepted July 6, 2018

Supplementary Tables. Acc. Nos. alignments of aa sequences of analysed proteins of virus isolates with identified equine host markers (these supplementary tables are also available in Excell format from the corresponding author F. Kostolanský, E-mail: virufkos@savba.sk) 




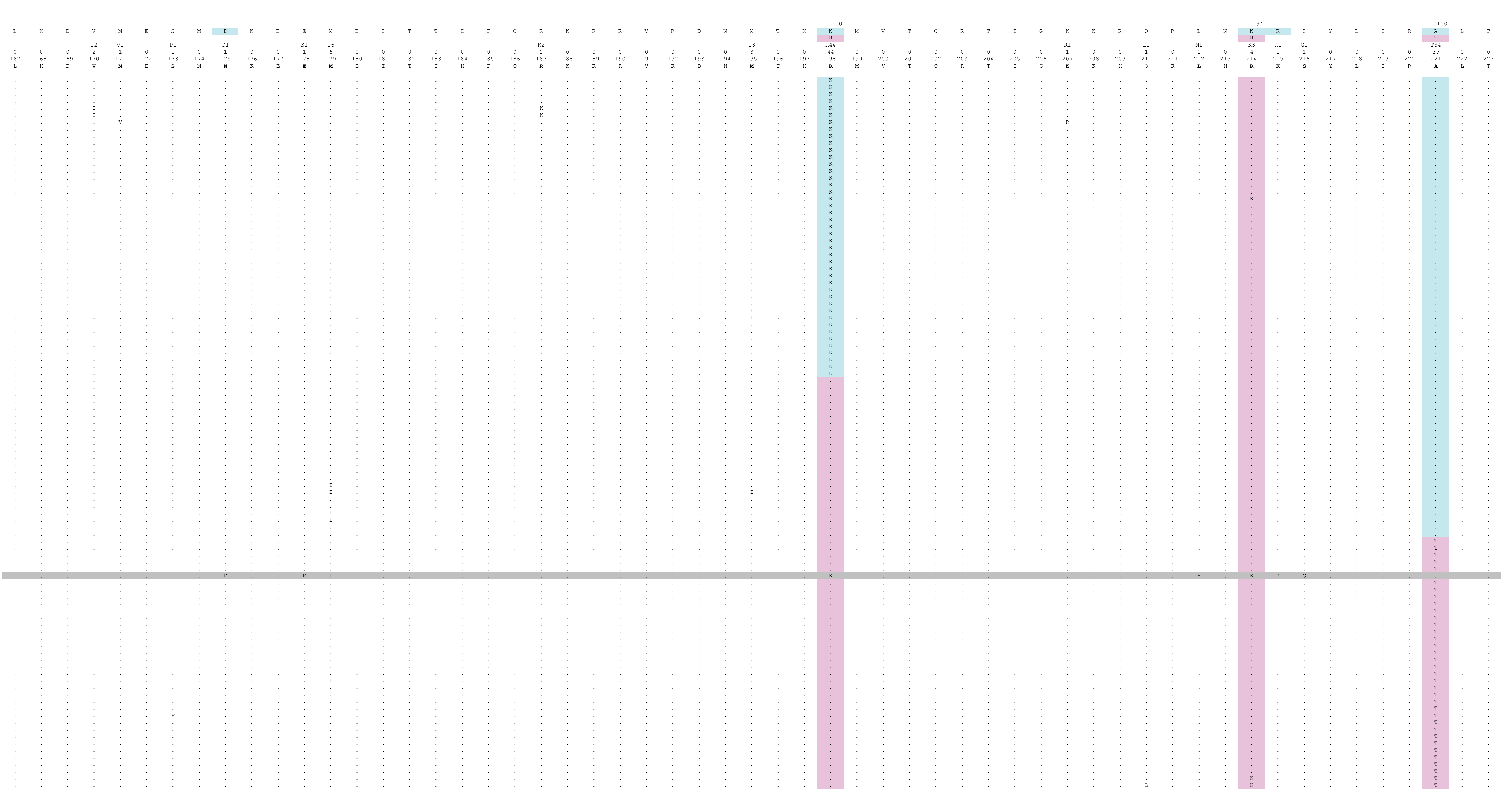




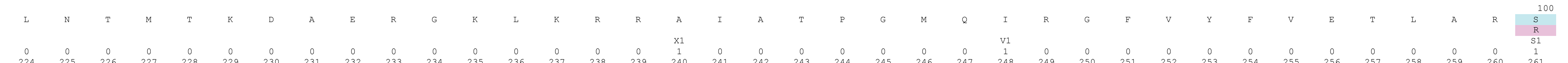

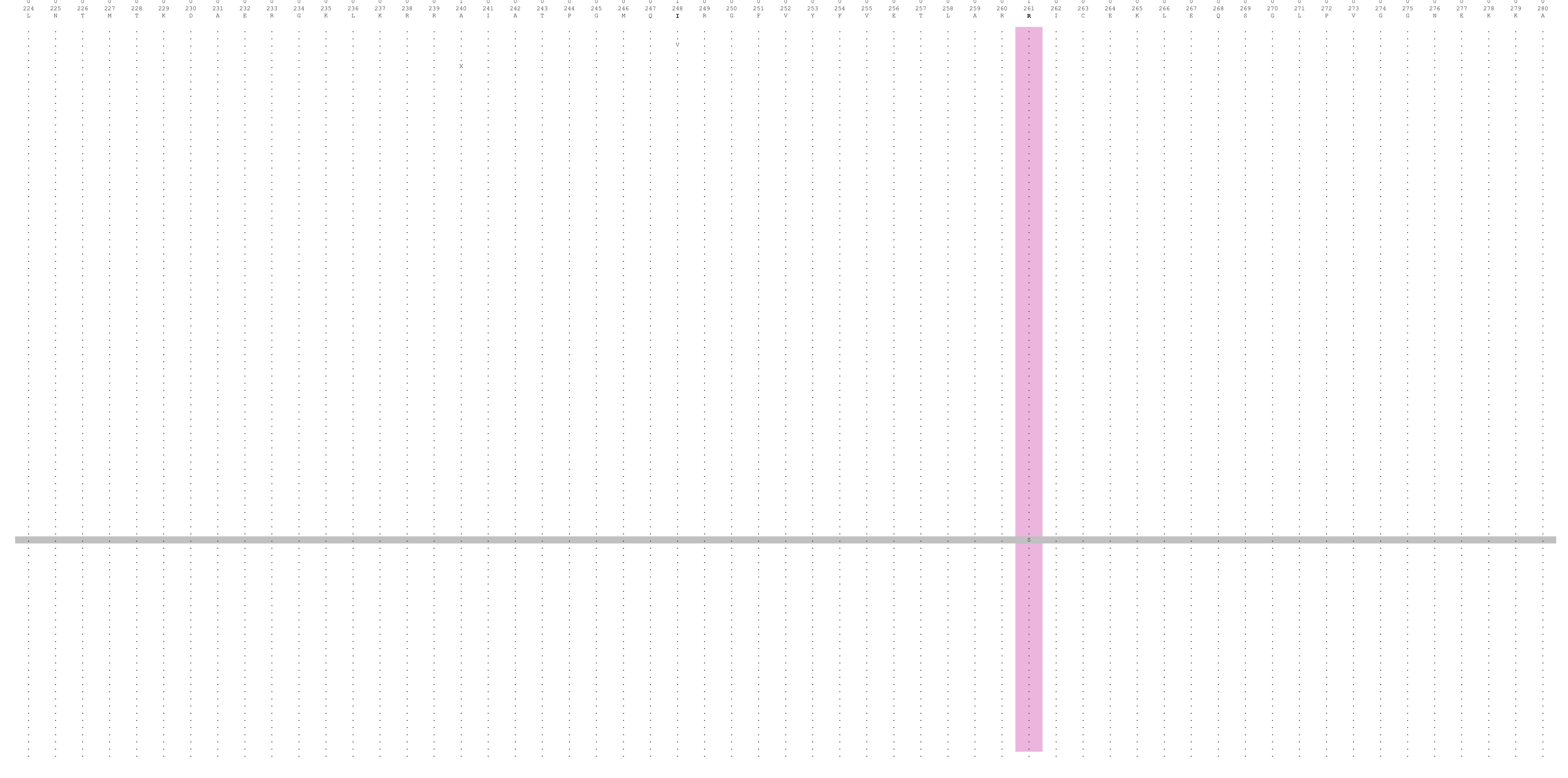




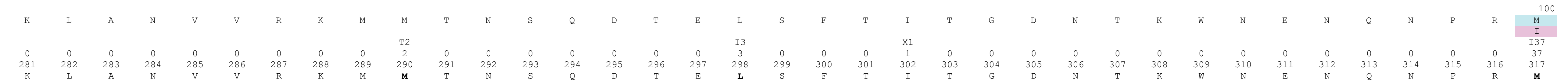

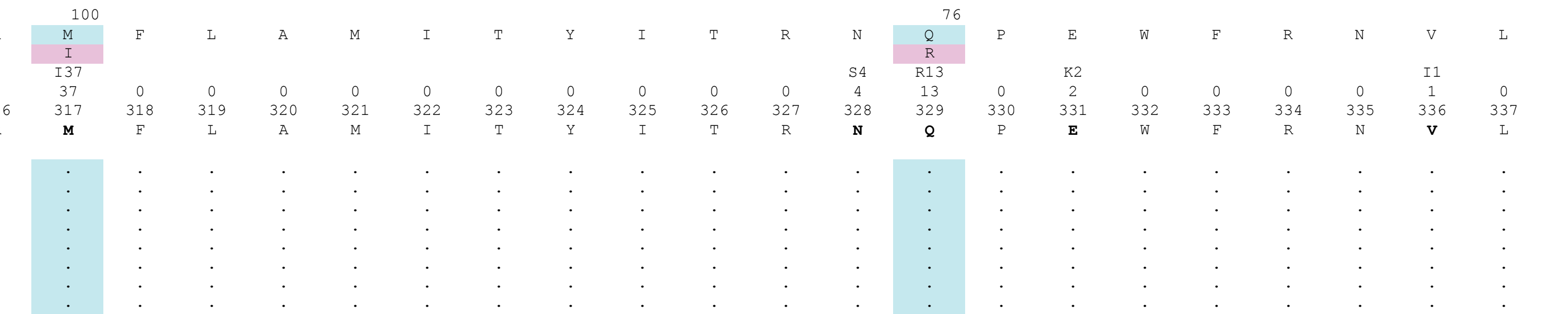




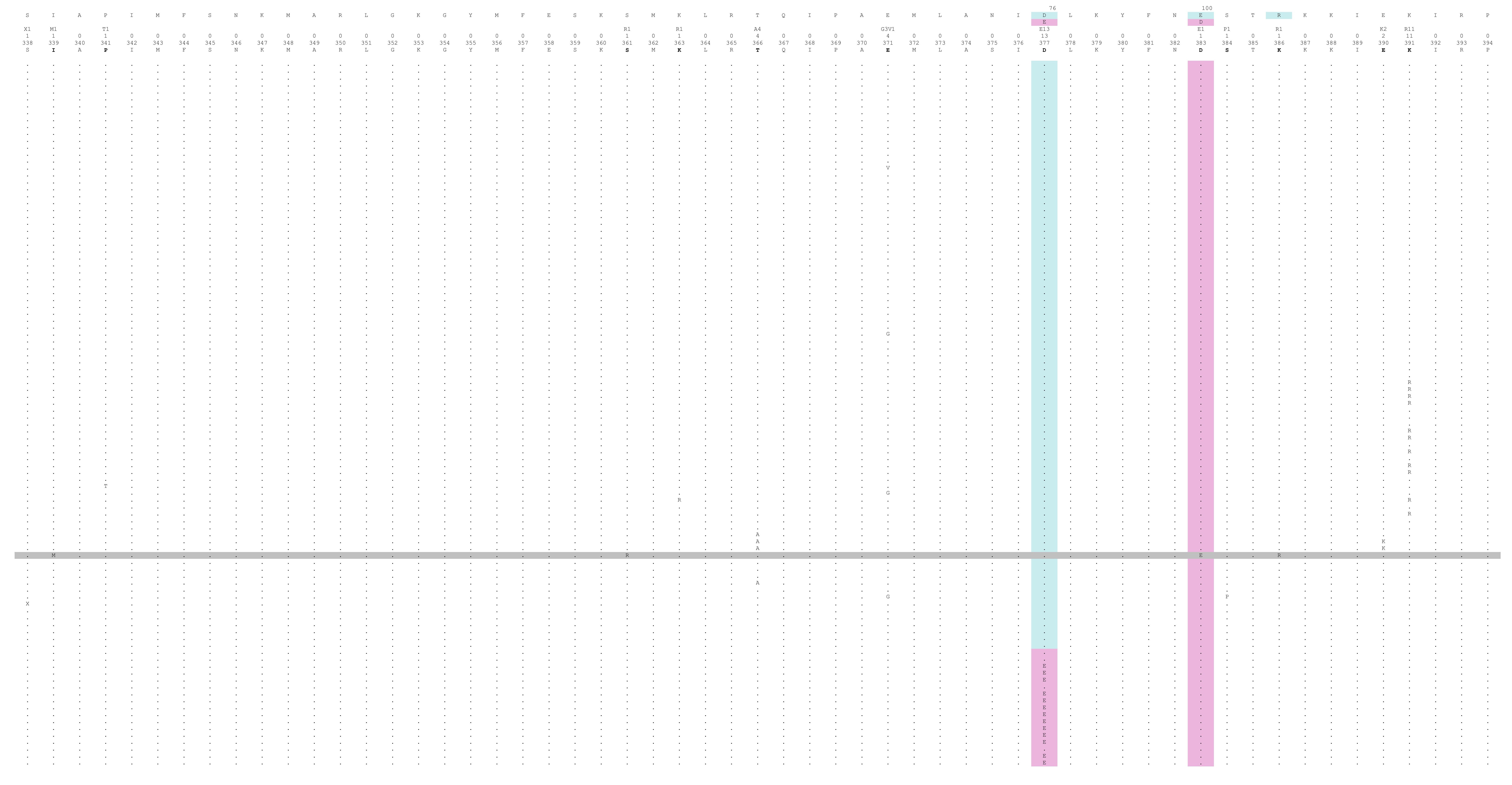




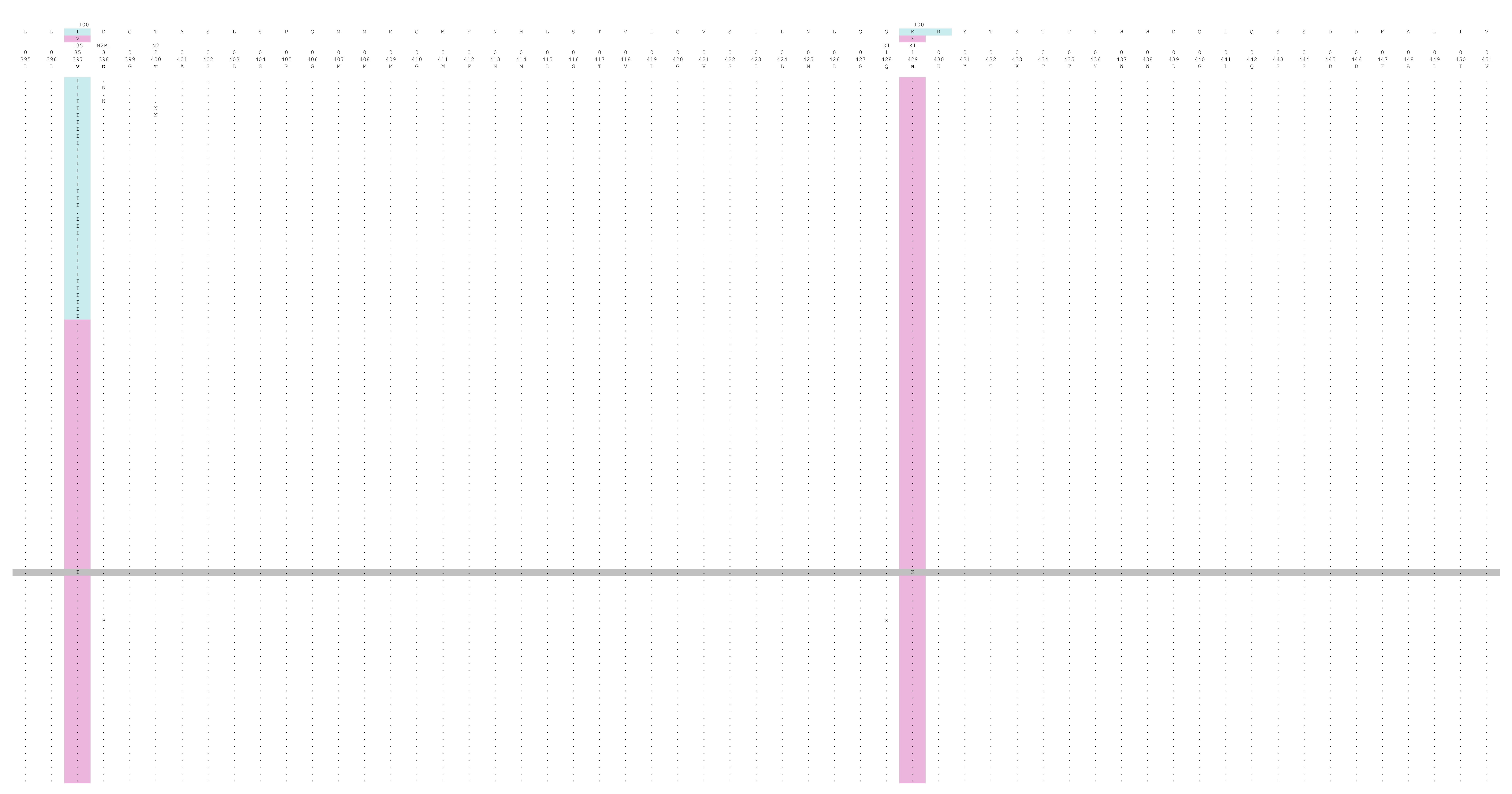




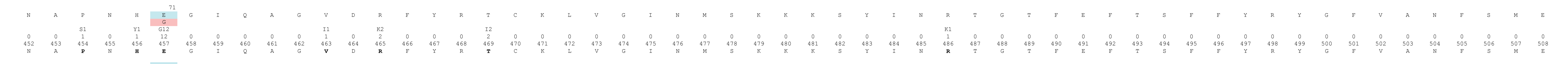

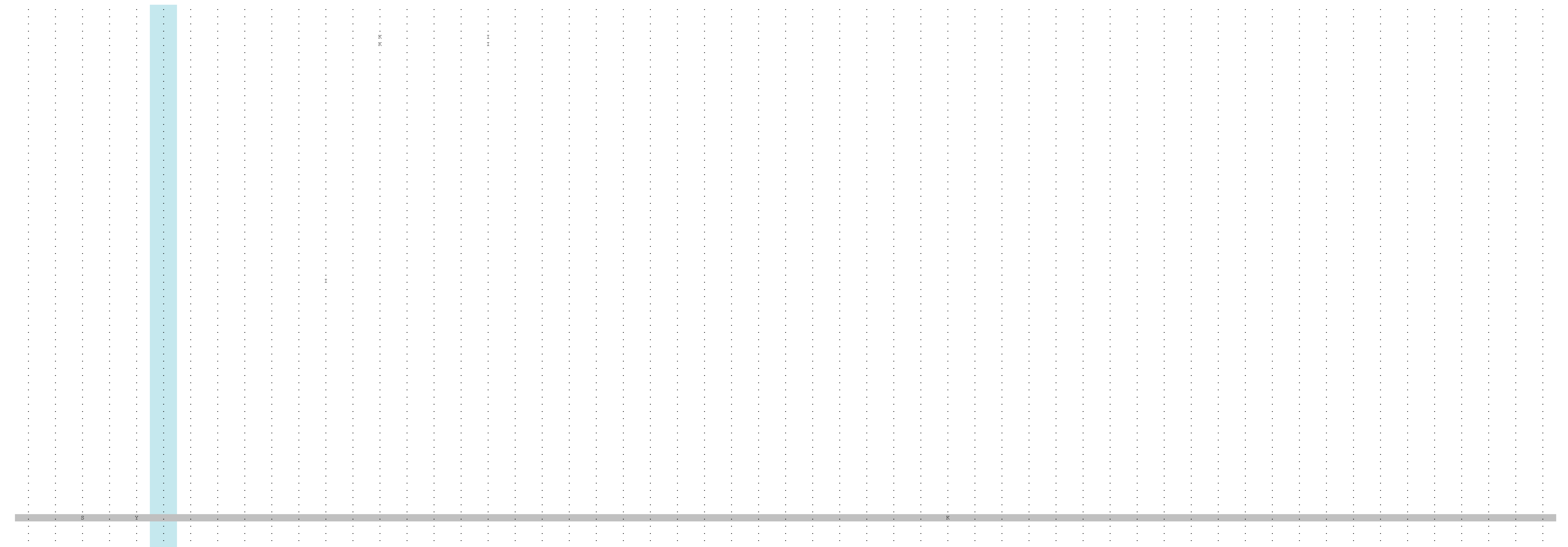




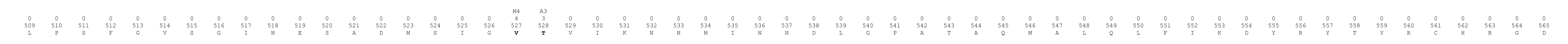




$$
\mid
$$




$$
1
$$




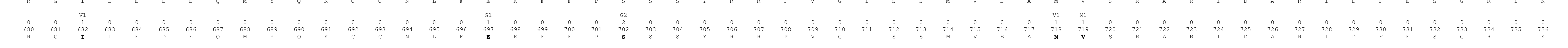




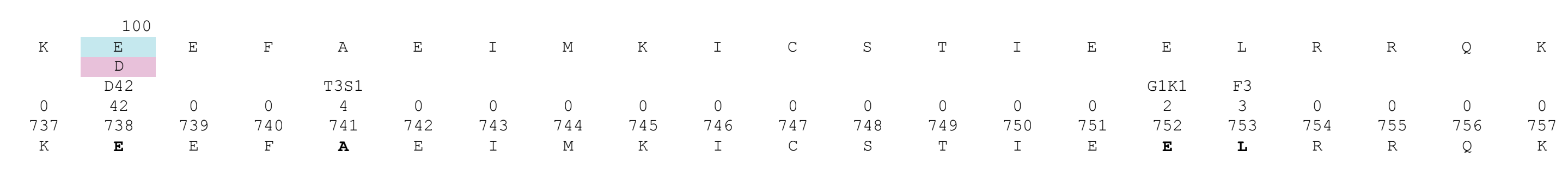


. Alternatives: aa different from consensus, e.g., Q1 means that Q occurred in one isolate Alignment Details: number of occurrences of various are of variability.

4. High-potency and Low-potency markers are colored green and yellow, respectively.

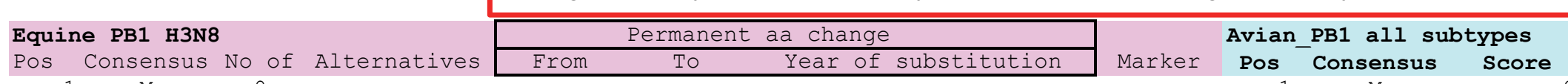


$1 \mathrm{R}=5, \mathrm{Q}=9601, \mathrm{xaa}=3$

$1 \mathrm{G}=9604, \mathrm{~S}=2, \mathrm{xaa}=3$

$1 \mathrm{R}=9604, \mathrm{C}=1, \mathrm{H}=1, \mathrm{Xaa}=3$

$1 \mathrm{R}=2, \mathrm{Q}=9604, \mathrm{Xaa}=3$

$1 \mathrm{I}=1, \mathrm{~T}=9605, \mathrm{Xaa}=3$

$1 \mathrm{~A}=1, \mathrm{D}=9602, \mathrm{G}=1, \mathrm{Xaa}=5$

$1 \mathrm{R}=1, \mathrm{~W}=9603, \mathrm{Xaa}=5$

$1 \mathrm{~A}=3, \mathrm{I}=1, \mathrm{~T}=9600, \mathrm{Xaa}=5$

$2 \mathrm{I}=4, \mathrm{~L}=9593, \mathrm{M}=3, \mathrm{~S}=2, \mathrm{~V}=2, \mathrm{Xaa}=5$

$3 \mathrm{~N}=9581, \mathrm{D}=17, \mathrm{I}=2, \mathrm{~K}=2, \mathrm{~S}=1, \mathrm{Xaa}=6$

$2 \mathrm{R}=9596, \mathrm{E}=1, \mathrm{~K}=5, \mathrm{~S}=1, \mathrm{Xaa}=6$

$1 \mathrm{~N}=9601, \mathrm{~S}=2, \mathrm{Xaa}=6$

$\mathrm{A}=1, \mathrm{R}=1, \mathrm{I}=1, \mathrm{P}=9598, \mathrm{~T}=1, \mathrm{Xaa}=7$

$1 \mathrm{~A}=9598, \mathrm{G}=2, \mathrm{P}=2, \mathrm{Xaa}=7$

$1 \mathrm{~A}=9598, \mathrm{~S}=3, \mathrm{~T}=1, \mathrm{Xaa}=7$

$1 \mathrm{~A}=2, \mathrm{~S}=1, \mathrm{~T}=9598, \mathrm{Xaa}=8$

$1 \mathrm{~A}=9600, \mathrm{D}=1, \mathrm{~V}=1, \mathrm{xaa}=7$

$1 \mathrm{~L}=9601, \mathrm{~F}=1, \mathrm{Xaa}=7$

$2 \mathrm{~N}=9592, \mathrm{D}=5, \mathrm{~S}=5, \mathrm{Xaa}=7$

$2 \mathrm{~A}=3, \mathrm{I}=1, \mathrm{P}=1, \mathrm{~S}=1, \mathrm{~T}=9595, \mathrm{Xaa}=8$

$2 \mathrm{I}=9597, \mathrm{M}=2, \mathrm{~T}=1, \mathrm{~V}=1, \mathrm{Xa} a=8$.

$2 \mathrm{D}=1, \mathrm{Q}=1, \mathrm{E}=9595, \mathrm{G}=3, \mathrm{~K}=1, \mathrm{Xaa}=8$

$57 \mathrm{I}=1235, \mathrm{~T}=3, \mathrm{~V}=8363, \mathrm{Xaa}=8$

$2 \mathrm{~L}=3, \mathrm{~F}=9596, \mathrm{~S}=1, \mathrm{Y}=1, \mathrm{Xaa}=8$

$17 \mathrm{~A}=15, \mathrm{~L}=166, \mathrm{M}=1, \mathrm{P}=1, \mathrm{~S}=9401, \mathrm{~T}=17, \mathrm{Xaa}=8$

$1 \mathrm{~N}=9599, \mathrm{D}=1, \mathrm{~S}=1, \mathrm{Xaa}=8$

$27 \mathrm{~N}=7, \mathrm{D}=150, \mathrm{C}=5, \mathrm{G}=9271, \mathrm{~S}=168$, Xaa $=8$

$3 \mathrm{I}=9, \mathrm{~L}=9580, \mathrm{M}=11, \mathrm{P}=1, \mathrm{xaa}=8$

$20 \mathrm{~A}=117, \mathrm{I}=17, \mathrm{~K}=24, \mathrm{M}=32, \mathrm{~S}=3, \mathrm{~T}=9398, \mathrm{~V}=10, \mathrm{Xaa}=8$

$22 \mathrm{~A}=9354, \mathrm{~N}=13, \mathrm{D}=2, \mathrm{I}=17, \mathrm{~S}=19, \mathrm{~T}=176, \mathrm{~V}=20, \mathrm{Xa} a=8$

$19 \mathrm{~N}=9343, \mathrm{~S}=258, \mathrm{Xaa}=8$

$9 \mathrm{D}=56, \mathrm{E}=9518, \mathrm{G}=19, \mathrm{~K}=4, \mathrm{~V}=3, \mathrm{Xaa}=9$

$1 \mathrm{~K}=1, \mathrm{~S}=9599, \mathrm{~T}=1, \mathrm{Xaa}=8$

$1 \mathrm{G}=9601, \mathrm{Xaa}=8$

$2 \mathrm{R}=9596, \mathrm{P}=1, \mathrm{~S}=2, \mathrm{~T}=2, \mathrm{Xaa}=8$

$1 \mathrm{~L}=9599, \mathrm{M}=2, \mathrm{Xaa}=8$

$6 \mathrm{I}=9556, \mathrm{~L}=3, \mathrm{M}=24, \mathrm{~V}=18, \mathrm{xaa}=8$

$2 \mathrm{~A}=1, \mathrm{~N}=2, \mathrm{D}=9596, \mathrm{E}=1, \mathrm{Y}=1, \mathrm{Xaa}=8$

$4 \mathrm{I}=1, \mathrm{~L}=16, \mathrm{~F}=9578, \mathrm{~S}=4, \mathrm{Y}=1$, $\mathrm{Xa} a=9$

$1 \mathrm{~L}=9599, \mathrm{~V}=2, \mathrm{Xaa}=8$

$3 \mathrm{R}=24, \mathrm{~K}=9577, \mathrm{Xaa}=8$

$2 \mathrm{R}=1, \mathrm{~N}=2, \mathrm{D}=9596, \mathrm{E}=1, \mathrm{G}=1, \mathrm{Xaa}=8$

$2 \mathrm{~A}=2, \mathrm{G}=1, \mathrm{I}=4, \mathrm{M}=1, \mathrm{~V}=9593, \mathrm{Xaa}=8$

$29 \mathrm{~A}=2, \mathrm{Del}=1, \mathrm{I}=115, \mathrm{~L}=15, \mathrm{M}=9268, \mathrm{~T}=41, \mathrm{~V}=159, \mathrm{xaa}=8$.

$32 \mathrm{~N}=1, \mathrm{D}=318$, Del=1, $\mathrm{Q}=1, \mathrm{E}=9182, \mathrm{G}=68, \mathrm{~K}=28, \mathrm{~V}=2, \mathrm{Xaa}=8$

$1 \mathrm{Del}=1, \mathrm{~L}=1, \mathrm{~S}=9600, \mathrm{Xaa}=7$

10 Del=1, $I=36, \mathrm{~L}=2, \mathrm{M}=9517, \mathrm{~T}=9, \mathrm{~V}=37, \mathrm{Xaa}=7$

$33 \mathrm{~N}=484, \mathrm{D}=9086, \mathrm{E}=10, \mathrm{G}=20, \mathrm{Y}=1, \mathrm{Xaa}=8$

$8 \mathrm{R}=50, \mathrm{~N}=7, \mathrm{Q}=4, \mathrm{E}=4, \mathrm{~K}=9536, \mathrm{Xa}=8$

$21 \mathrm{~A}=2, \mathrm{R}=1, \mathrm{~N}=10, \mathrm{D}=62, \mathrm{Q}=1, \mathrm{E}=9387, \mathrm{G}=82, \mathrm{~K}=57, \mathrm{Xaa}=7$

$22 \mathrm{~A}=3, \mathrm{D}=10, \mathrm{E}=9339, \mathrm{G}=147, \mathrm{~K}=102, \mathrm{~V}=1, \mathrm{Xaa}=7$

$39 \mathrm{I}=314, \mathrm{~L}=25, \mathrm{~K}=1, \mathrm{M}=9058, \mathrm{~T}=5, \mathrm{~V}=199, \mathrm{Xaa}=7$

$6 \mathrm{D}=11, \mathrm{E}=9555, \mathrm{G}=31, \mathrm{~K}=4, \mathrm{~V}=1$, $\mathrm{Xaa}=7$

$6 \mathrm{I}=9555, \mathrm{~L}=1, \mathrm{M}=4, \mathrm{~T}=1, \mathrm{~V}=40, \mathrm{Xaa}=8$

$24 \mathrm{~A}=3, \mathrm{R}=1, \mathrm{I}=330, \mathrm{~T}=9258, \mathrm{~V}=10$, $\mathrm{Xaa}=7$

$4 \mathrm{~A}=21, \mathrm{I}=3, \mathrm{M}=2, \mathrm{~T}=9576, \mathrm{Xaa}=7$

$2 \mathrm{R}=1, \mathrm{Q}=4, \mathrm{H}=9595, \mathrm{P}=1, \mathrm{Y}=1, \mathrm{X} a \mathrm{a}=7$

$1 \mathrm{~L}=1, \mathrm{~F}=9601, \mathrm{Xaa}=7$

$3 \mathrm{Q}=9581, \mathrm{H}=20, \mathrm{P}=1, \mathrm{Xaa}=$

$1 \mathrm{R}=9597, \mathrm{~K}=4, \mathrm{Xaa}=8$

$2 \mathrm{R}=3, \mathrm{~N}=1, \mathrm{~K}=9596, \mathrm{M}=1, \mathrm{Xaa}=8$

$3 \mathrm{R}=9587, \mathrm{G}=1, \mathrm{I}=1, \mathrm{~K}=11, \mathrm{~S}=1, \mathrm{Xaa}=8$

$2 \mathrm{R}=9595, \mathrm{I}=1, \mathrm{~K}=5, \mathrm{Xaa}=8$

$15 \mathrm{I}=166, \mathrm{~L}=4, \mathrm{M}=11, \mathrm{~V}=9420, \mathrm{xaa}=8$

$2 \mathrm{R}=9593, \mathrm{G}=1, \mathrm{~K}=5, \mathrm{Xaa}=10$

$2 \mathrm{~A}=1, \mathrm{D}=9590, \mathrm{E}=2, \mathrm{G}=3, \mathrm{H}=2, \mathrm{Y}=1, \mathrm{Xaa}=10$

$5 \mathrm{~N}=9567, \mathrm{~S}=28, \mathrm{~T}=2, \mathrm{Y}=2, \mathrm{Xaa}=10$

$9 \mathrm{I}=48, \mathrm{I}=2, \mathrm{M}=9526, \mathrm{~T}=7, \mathrm{~V}=16, \mathrm{Xaa}=10$

$2 \mathrm{~A}=1, \mathrm{I}=1, \mathrm{P}=2, \mathrm{~S}=4, \mathrm{~T}=9591, \mathrm{Xaa}=10$

$8 \mathrm{R}=67, \mathrm{~N}=2, \mathrm{Q}=2, \mathrm{I}=1, \mathrm{~K}=9524, \mathrm{~T}=1$, $\mathrm{Xaa}=12$

$6 \mathrm{R}=46, \mathrm{~N}=1, \mathrm{Q}=1, \mathrm{E}=1, \mathrm{~K}=9549$, Xaa $=1$

$2 \mathrm{I}=1, \mathrm{~L}=4, \mathrm{M}=9589, \mathrm{~V}=4, \mathrm{Xaa}=11$

$32 \mathrm{~A}=3, \mathrm{I}=509, \mathrm{~F}=2, \mathrm{~T}=1, \mathrm{~V}=9083, \mathrm{Xaa}=11$

$2 \mathrm{~A}=3, \mathrm{R}=1, \mathrm{I}=1, \mathrm{P}=2, \mathrm{~S}=1, \mathrm{~T}=9590, \mathrm{Xaa}=11$

$2 \mathrm{R}=5, \mathrm{Q}=9590, \mathrm{H}=1, \mathrm{P}=1, \mathrm{Xaa}=12$

$3 \mathrm{R}=9588, \mathrm{G}=3, \mathrm{~K}=4, \mathrm{~S}=2, \mathrm{Xaa}=12$

$3 \mathrm{~A}=2, \mathrm{I}=8, \mathrm{~K}=1, \mathrm{P}=3, \mathrm{~S}=2, \mathrm{~T}=9581$, $\mathrm{Xaa}=12$

$8 \mathrm{R}=1, \mathrm{I}=9527, \mathrm{~L}=10, \mathrm{M}=6, \mathrm{~V}=53, \mathrm{Xaa}=12$

$2 \mathrm{~A}=1, \mathrm{R}=2, \mathrm{E}=1, \mathrm{G}=9592, \mathrm{~V}=1, \mathrm{xaa}=12$

$6 \mathrm{R}=39, \mathrm{~N}=1, \mathrm{E}=1, \mathrm{~K}=9555, \mathrm{~T}=1, \mathrm{Xaa}=12$

$3 \mathrm{R}=9, \mathrm{E}=4, \mathrm{~K}=9584, \mathrm{Xaa}=12$

$7 \mathrm{R}=53, \mathrm{~N}=4, \mathrm{E}=3, \mathrm{I}=1, \mathrm{~K}=9536, \mathrm{Xaa}=12$

$4 \mathrm{Q}=9577, \mathrm{H}=12, \mathrm{~L}=4, \mathrm{~K}=4, \mathrm{Xaa}=12$

$27 \mathrm{~A}=3, \mathrm{R}=9270, \mathrm{E}=3, \mathrm{G}=93, \mathrm{X}=224, \mathrm{M}=4, \mathrm{Xa} \mathrm{a}=12$

$6 \mathrm{I}=14, \mathrm{~L}=9555, \mathrm{M}=14, \mathrm{~V}=14, \mathrm{Xa} a=12$

$29 \mathrm{~A}=2, \mathrm{~N}=9277, \mathrm{D}=12, \mathrm{G}=2, \mathrm{H}=3, \mathrm{I}=36, \mathrm{~K}=41, \mathrm{~S}=153, \mathrm{~T}=68, \mathrm{Y}=3, \mathrm{Xaa}=12$

$26 \mathrm{R}=341, \mathrm{~N}=19, \mathrm{Q}=4, \mathrm{I}=1, \mathrm{~K}=9231$, $\mathrm{Xa} a=13$

$35 \mathrm{~A}=1, \mathrm{R}=2, \mathrm{~N}=228, \mathrm{C}=3, \mathrm{E}=1, \mathrm{G}=206, \mathrm{I}=8, \mathrm{~S}=9147, \mathrm{~T}=1, \mathrm{Xaa}=12$

$2 \mathrm{C}=3, \mathrm{H}=4, \mathrm{~L}=1, \mathrm{Y}=9589, \mathrm{Xaa}=12$

$3 \mathrm{I}=8, \mathrm{~L}=9586, \mathrm{M}=1, \mathrm{~F}=1, \mathrm{~V}=1, \mathrm{Xa} a=12$

$3 \mathrm{G}=1, \mathrm{I}=9581, \mathrm{M}=1, \mathrm{~T}=4, \mathrm{~V}=9, \mathrm{Xaa}=13$

$2 \mathrm{R}=9595, \mathrm{~K}=2, \mathrm{Xaa}=12$

$3 \mathrm{~A}=9589, \mathrm{G}=2, \mathrm{~S}=2, \mathrm{~T}=4, \mathrm{~V}=1, \mathrm{Xaa}=11$

$3 \mathrm{~L}=9588, \mathrm{M}=8, \mathrm{~S}=1, \mathrm{~V}=1, \mathrm{X} a \mathrm{a}=11$

$4 \mathrm{~A}=31, \mathrm{~T}=9567, \mathrm{Xaa}=1$

$1 \mathrm{~L}=9598, \mathrm{Xaa}=11$

$1 \mathrm{~N}=9597, \mathrm{~S}=1, \mathrm{Xaa}=11$

$2 \mathrm{~A}=1, \mathrm{~K}=1, \mathrm{P}=1, \mathrm{~T}=9595, \mathrm{Xaa}=11$

$2 \mathrm{I}=1, \mathrm{~K}=1, \mathrm{M}=9595, \mathrm{~T}=1, \mathrm{Xaa}=1$

$2 \mathrm{~A}=4, \mathrm{I}=1, \mathrm{~S}=1, \mathrm{~T}=9592, \mathrm{Xaa}=1$

$2 \mathrm{R}=1, \mathrm{E}=1, \mathrm{~K}=9596, \mathrm{Xaa}=11$

$2 \mathrm{~N}=5, \mathrm{D}=9592, \mathrm{G}=1, \mathrm{X}$

$1 \mathrm{~A}=9598, \mathrm{Xaa}=1$

$2 \mathrm{Q}=1, \mathrm{E}=9594, \mathrm{~K}=2, \mathrm{Xaa}=12$

$2 \mathrm{R}=9595, \mathrm{G}=2, \mathrm{~T}=1, \mathrm{Xaa}=11$

$2 \mathrm{R}=1, \mathrm{G}=9594, \mathrm{~S}=2, \mathrm{xaa}=12$

$2 \mathrm{~N}=1, \mathrm{E}=1, \mathrm{~K}=9595, \mathrm{Xaa}=12$

$\mathrm{R}=80, \mathrm{~N}=1, \mathrm{E}=3, \mathrm{G}=1, \mathrm{~K}=9510, \mathrm{M}=2, \mathrm{Xaa}=12$

$2 \mathrm{R}=9591, \mathrm{~K}=6, \mathrm{Xaa}=12$

$1 \mathrm{R}=9597, \mathrm{Xaa}=12$

$2 \mathrm{~A}=9595, \mathrm{De} 1=1, \mathrm{E}=1, \mathrm{~T}=1, \mathrm{Xaa}=11$ 
$2 \mathrm{P}=9595, \mathrm{~S}=1, \mathrm{Xaa}=13$
$2 \mathrm{G}=9594, \mathrm{P}=1, \mathrm{Xa}=14$

$4 \mathrm{R}=1, \mathrm{I}=19, \mathrm{M}=9574, \mathrm{~V}=1, \mathrm{Xaa}=14$

$2 \mathrm{R}=1, \mathrm{Q}=9593, \mathrm{~T}=1, \mathrm{Xaa}=14$

$3 \mathrm{~N}=1, \mathrm{I}=9585, \mathrm{~L}=1, \mathrm{~F}=2, \mathrm{~T}=6, \mathrm{Xaa}=14$

$3 \mathrm{R}=9586, \mathrm{~K}=3, \mathrm{~T}=1, \mathrm{Xaa}=19$

$2 \mathrm{G}=9595, \mathrm{Xaa}=14$

$2 \mathrm{I}=1, \mathrm{~F}=9589, \mathrm{~S}=3, \mathrm{Xaa}=16$

$10 \mathrm{C}=1, \mathrm{H}=70, \mathrm{~F}=16, \mathrm{Y}=9507, \mathrm{Xaa}=15$

$4 \mathrm{~L}=2, \mathrm{~F}=9579, \mathrm{~S}=4, \mathrm{~V}=9, \mathrm{Xaa}=15$

$3 \mathrm{~A}=1, \mathrm{I}=7, \mathrm{~V}=9586, \mathrm{Xaa}=15$

$23 \mathrm{~A}=263, \mathrm{I}=13, \mathrm{M}=6, \mathrm{~S}=4, \mathrm{~T}=9308, \mathrm{Xaa}=15$

$2 \mathrm{I}=1, \mathrm{~L}=9590, \mathrm{M}=3, \mathrm{Xaa}=15$

$2 \mathrm{~A}=9591, \mathrm{P}=1, \mathrm{~T}=1, \mathrm{~V}=1, \mathrm{Xaa}=15$
$\mathrm{~A}=9574, G=2, \mathrm{~K}=17, \mathrm{~W}=1, \mathrm{Xa}=15$

$20 \mathrm{~A}=13, \mathrm{~N}=29, \mathrm{C}=91, \mathrm{G}=31, \mathrm{~S}=9404, \mathrm{~T}=25, \mathrm{Xaa}=16$

$2 \mathrm{I}=9588, \mathrm{M}=1, \mathrm{~T}=1, \mathrm{~V}=3, \mathrm{Xaa}=16$

$2 \mathrm{C}=9593, \mathrm{Xaa}=16$

4. $\mathrm{D}=18, \mathrm{E}=9574, \mathrm{Xaa}=17$

$2 \mathrm{I}=1, \mathrm{~L}=9590, \mathrm{~V}=1, \mathrm{Xaa}=17$

$2 \mathrm{D}=1, \mathrm{Q}=1, \mathrm{E}=9588, \mathrm{G}=2, \mathrm{Xaa}=17$

$2 \mathrm{R}=1, \mathrm{Q}=9590, \mathrm{P}=1, \mathrm{Xaa}=17$

$3 \mathrm{~A}=4, \mathrm{~F}=2, \mathrm{~S}=9584, \mathrm{~T}=1, \mathrm{Xaa}=18$

$2 \mathrm{~L}=9588, \mathrm{~F}=2, \mathrm{Xaa}=19$

$4 \mathrm{G}=1, \mathrm{I}=10, \mathrm{~F}=1, \mathrm{~V}=9578, \mathrm{Xaa}=19$

$2 \mathrm{R}=1, \mathrm{G}=9589, \mathrm{Xaa}=19$

$2 \mathrm{R}=1, \mathrm{G}=9589, \mathrm{Xaa}=19$
$2 \mathrm{~N}=9588, \mathrm{~K}=1, \mathrm{M}=1, \mathrm{Xaa}=19$

$2 \mathrm{D}=1, \mathrm{E}=9588, \mathrm{~K}=1, \mathrm{Xaa}=19$

2 $\mathrm{R}=1, \mathrm{~K}=9589, \mathrm{Xaa}=19$
$3 \mathrm{~N}=2, \mathrm{~K}=9586, \mathrm{M}=1, \mathrm{xaa}=20$

$2 \mathrm{~A}=9587, \mathrm{G}=3, \mathrm{Xaa}=19$

$\mathrm{A}=9587, \mathrm{~N}=1, \mathrm{~K}=9588, \mathrm{Xaa}=20$
$\mathrm{I}=9588, \mathrm{Xa}=21$

$2 \mathrm{~L}=9588, \mathrm{Xaa}=21$
$3 \mathrm{~A}=9584, \mathrm{G}=4, \mathrm{~S}=1, \mathrm{Xaa}=20$

$2 \mathrm{~N}=9587, \mathrm{~S}=1, \mathrm{Xaa}=21$
$100 \mathrm{Del}=3943, \mathrm{~V}=5645, \mathrm{Xaa}$

$5 \mathrm{~A}=18, \mathrm{G}=1, \mathrm{I}=4, \mathrm{~V}=9564, \mathrm{Xaa}=22$

$5 \mathrm{~A}=18, \mathrm{G}=1, \mathrm{I}=4, \mathrm{~V}=9564, \mathrm{X} a \mathrm{a}=22$
$3 \mathrm{R}=9583, \mathrm{I}=1, \mathrm{~K}=3, \mathrm{Xa} a=22$

$2 \mathrm{~K}=9587, \mathrm{Xaa}=22$
$2 \mathrm{M}=9587, \mathrm{Xaa}=22$

$2 \mathrm{M}=9587, \mathrm{Xaa}=22$
$3 \mathrm{I}=1, \mathrm{M}=9585, \mathrm{Xaa}=23$

$\mathrm{A}=9, \mathrm{I}=3, \mathrm{~T}=9573, \mathrm{~V}=1, \mathrm{Xaa}=23$

$6 \mathrm{~A}=1, \mathrm{~N}=9550, \mathrm{D}=10, \mathrm{~K}=1, \mathrm{~S}=25, \mathrm{Xaa}=22$

$3 \mathrm{~A}=1, \mathrm{~L}=1, \mathrm{P}=1, \mathrm{~S}=9584, \mathrm{Xaa}=22$
$\mathrm{R}=22, \mathrm{Q}=9532, \mathrm{H}=19, \mathrm{~L}=6, \mathrm{~K}=8, \mathrm{Xaa}=22$

$3 \mathrm{D}=9584, \mathrm{E}=2, \mathrm{Y}=1, \mathrm{~V}=1, \mathrm{Xaa}=21$

$4 \mathrm{~A}=5, \mathrm{~N}=1, \mathrm{I}=8, \mathrm{~S}=2, \mathrm{~T}=9572, \mathrm{Xaa}=21$

$=1, v=25, x a a=21$

$\mathrm{S}=9585, \mathrm{~T}=1, \mathrm{Xaa}=23$

A $\mathrm{A}=1, \mathrm{~S}=1, \mathrm{~T}=9586$, Хaa $=2$

$\mathrm{I}=9537, \mathrm{~T}=2, \mathrm{~V}=48, \mathrm{Xaa}=22$

$\mathrm{I}=2, \mathrm{~T}=9585, \mathrm{Xaa}=22$
$\mathrm{C}=9587, \mathrm{Xa}=22$

$\mathrm{D}=9585, \mathrm{G}=1, \mathrm{~K}=1, \mathrm{Xaa}=22$

$\mathrm{N}=9583, \mathrm{H}=1, \mathrm{~K}=2, \mathrm{~S}=1, \mathrm{Xaa}=22$

$2 \mathrm{~A}=1, \mathrm{~T}=9586, \mathrm{Xaa}=22$
$3 \mathrm{~K}=9585, \mathrm{~T}=1, \mathrm{Xaa}=23$

$3 \mathrm{~K}=9585, \mathrm{~T}=1, \mathrm{Xaa}=23$
$3 \mathrm{R}=2, \mathrm{~W}=9583, \mathrm{Xaa}=24$

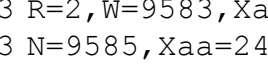

$\mathrm{D}=1, \mathrm{E}=9584, \mathrm{xaa}=24$

$3 \mathrm{~N}=9583, \mathrm{Xaa}=26$
$\mathrm{Q}=9581, \mathrm{H}=1, \mathrm{P}=1, \mathrm{Xaa}=26$

$13 \mathrm{~N}=9461, \mathrm{~K}=1, \mathrm{~S}=122, \mathrm{Xaa}=25$

3 $\mathrm{P}=9583, \mathrm{~S}=1, \mathrm{Xaa}=25$

$47 \mathrm{~A}=1, \mathrm{R}=1, \mathrm{I}=244, \mathrm{M}=8919, \mathrm{~T}=34, \mathrm{~V}=384, \mathrm{Xa}=26$

F $F=9583, \mathrm{Xaa}=26$

$5 \mathrm{~L}=9563, \mathrm{M}=20$, Xaa $=26$

$22 \mathrm{~A}=9304, \mathrm{~S}=4, \mathrm{~T}=272, \mathrm{~V}=2, \mathrm{xaa}=27$

R $\mathrm{R}=1, \mathrm{I}=4, \mathrm{M}=9576, \mathrm{Xaa}=28$

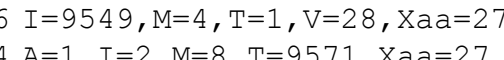

$4 \mathrm{~F}=11, \mathrm{Y}=9571$, $\mathrm{Xa} a=27$

$4 \mathrm{I}=9572, \mathrm{M}=7, \mathrm{~T}=2, \mathrm{x}$
$3 \mathrm{~T}=9580, \mathrm{Xaa}=29$

$\mathrm{R}=9508, \mathrm{~K}=71, \mathrm{~W}=1, \mathrm{Xaa}=29$

, $=7, G=1, H=10, K=64, S=77, T=9, Y=2, X a a=29$

01 Del $=3943, Q=5635, \mathrm{~K}=1, \mathrm{Xaa}=30$

$22 \mathrm{H}=1, \mathrm{P}=9578, \mathrm{Xaa}=30 \mathrm{X}=1, \mathrm{E}=9371, \mathrm{G}=76, \mathrm{~K}=89, \mathrm{~V}=4, \mathrm{Xaa}=31$

$3 \mathrm{~W}=9577, \mathrm{Xaa}=32$

$4 \mathrm{~L}=9, \mathrm{~F}=9568, \mathrm{Xaa}=32$

$4 \mathrm{~N}=9573, \mathrm{D}=3, \mathrm{~T}=1, \mathrm{Xa} a=32$

$13 \mathrm{~A}=40, \mathrm{I}=61, \mathrm{~V}=9476, \mathrm{Xaa}=32$

$R=1, N=5, C=25, I=3, S=9542, T=2, x a a=31$

$11 \mathrm{I}=9500, \mathrm{M}=16, \mathrm{~T}=1$
$3 \mathrm{~A}=9578, \mathrm{Xaa}=31$

$3 \mathrm{~A}=9578, \mathrm{Xaa}=31$
$3 \mathrm{P}=9576, \mathrm{~S}=1, \mathrm{Xaa}=32$

$5 \mathrm{I}=9558, \mathrm{~L}=4, \mathrm{~V}=14, \mathrm{Xaa}=33$

$4 \mathrm{I}=3, \mathrm{M}=9575, \mathrm{Xa}=31$

$3 \mathrm{~F}=9578, \mathrm{~S}=1, \mathrm{Xaa}=30$

$3 \mathrm{~A}=1, \mathrm{~S}=9578, \mathrm{Xaa}=30$
$3 \mathrm{~N}=9577, \mathrm{D}=1, \mathrm{~K}=1, \mathrm{Xaa}=30$

$3 \mathrm{~K}=9579, \mathrm{Xaa}=30$

$6 \mathrm{I}=3, \mathrm{~L}=5, \mathrm{~K}=3, \mathrm{M}=9558, \mathrm{~V}=9, \mathrm{xaa}=31$

$4 \mathrm{~A}=9571, \mathrm{Del}=1, \mathrm{G}=3, \mathrm{~T}=3, \mathrm{~V}=2, \mathrm{Xaa}=29$

$6 \mathrm{R}=9550$, Del $=1, \mathrm{I}=3, \mathrm{~K}=26, \mathrm{Xaa}=29$

$4 \mathrm{Del}=1, \mathrm{I}=4, \mathrm{~L}=9575, \mathrm{Xaa}=29$

$28 \mathrm{R}=379, \mathrm{~N}=1, \mathrm{E}=2, \mathrm{~K}=9196, \mathrm{Xaa}=3$

$3 \mathrm{R}=1, \mathrm{G}=9579$, Xaa $=29$

$3 \mathrm{R}=1, \mathrm{G}=9579, \mathrm{Xa}=29$
$3 \mathrm{~S}=1, \mathrm{Y}=9579, \mathrm{Xa}=29$

$\mathrm{M}=9579, \mathrm{~T}=1, \mathrm{Xaa}=29$

I $\mathrm{I}=1, \mathrm{~L}=4, \mathrm{~F}=9575, \mathrm{Xaa}=29$

$5 \mathrm{~A}=1, \mathrm{D}=1, \mathrm{Q}=1, \mathrm{E}=9566, \mathrm{G}=2, \mathrm{~K}-\mathrm{X}$
$3 \mathrm{~N}=1, \mathrm{G}=1, \mathrm{~S}=9578, \mathrm{Xaa}=29$

$10 \mathrm{R}=58, \mathrm{~N}=10, \mathrm{E}=1, \mathrm{~K}=9511, \mathrm{Xaa}=29$

$16 \mathrm{R}=14, \mathrm{~N}=33, \mathrm{G}=28, \mathrm{I}=59, \mathrm{~S}=9444, \mathrm{~V}=1, \mathrm{Xaa}=30$

$\begin{array}{rl}4 & \mathrm{I}=1, \mathrm{~L}=6, \mathrm{M}=9573, \mathrm{Xaa}=29 \\ 13 \mathrm{R}=120, \mathrm{~K}=9458, \mathrm{M}=1, \mathrm{Xaa}=30\end{array}$

$7 \mathrm{H}=1, \mathrm{I}=33, \mathrm{~L}=9539, \mathrm{~F}=2, \mathrm{P}=1, \mathrm{~V}=2$, $\mathrm{Xaa}=3$

R $\mathrm{A}=26, \mathrm{I}=1, \mathrm{P}=1, \mathrm{~T}=95 \mathrm{a}, \mathrm{X}$

$4 \mathrm{Q}=9574, \mathrm{H}=1, \mathrm{~L}=1, \mathrm{Xaa}=33$

$28 \mathrm{I}=9206, \mathrm{~L}=1, \mathrm{M}=13, \mathrm{~T}=1, \mathrm{~V}=355, \mathrm{Xaa}=33$

$11 \mathrm{Q}=10, \mathrm{~L}=1, \mathrm{P}=9499, \mathrm{~S}=65, \mathrm{~T}=1, \mathrm{Xaa}=33$
$5 \mathrm{~A}=9559, \mathrm{~K}=1, \mathrm{~S}=2, \mathrm{~T}=12, \mathrm{~V}=2, \mathrm{X} a \mathrm{a}=33$

$5 \mathrm{~A}=9559, \mathrm{~K}=1, \mathrm{~S}=2, \mathrm{~T}=12, \mathrm{~V}=2, \mathrm{D}$
$4 \mathrm{D}=4, \mathrm{E}=9570, \mathrm{~K}=1$, хaа $=34$

$4 \mathrm{D}=4, \mathrm{~B}=9570, \mathrm{~K}=1, \mathrm{Xaa}=34 \mathrm{~V}, \mathrm{X}$

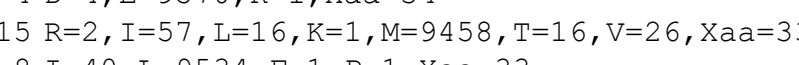

123 $\mathrm{N}=5843, \mathrm{D}=128, \mathrm{Del}=1, \mathrm{G}=10, \mathrm{H}=5, \mathrm{~K}=3, \mathrm{~S}=3340, \mathrm{~T}=244, \mathrm{Xaa}=35$

$\mathrm{A}=1, \mathrm{~N}=20, \mathrm{D}=9534, \mathrm{Del}=1, \mathrm{E}=16, \mathrm{G}=2, \mathrm{~T}=1, \mathrm{Xaa}=34$

$8 \mathrm{R}=35, \mathrm{~N}=3, \mathrm{Del}=1, \mathrm{Q}=4, \mathrm{I}=1, \mathrm{~K}=9528, \mathrm{~T}=1, \mathrm{Xaa}=36$

$101 \mathrm{Del}=3944, \mathrm{Y}=5630, \mathrm{Xaa}=35$

$4 \mathrm{Del}=1, \mathrm{~F}=9572, \mathrm{~S}=1, \mathrm{Xaa}=35$ 


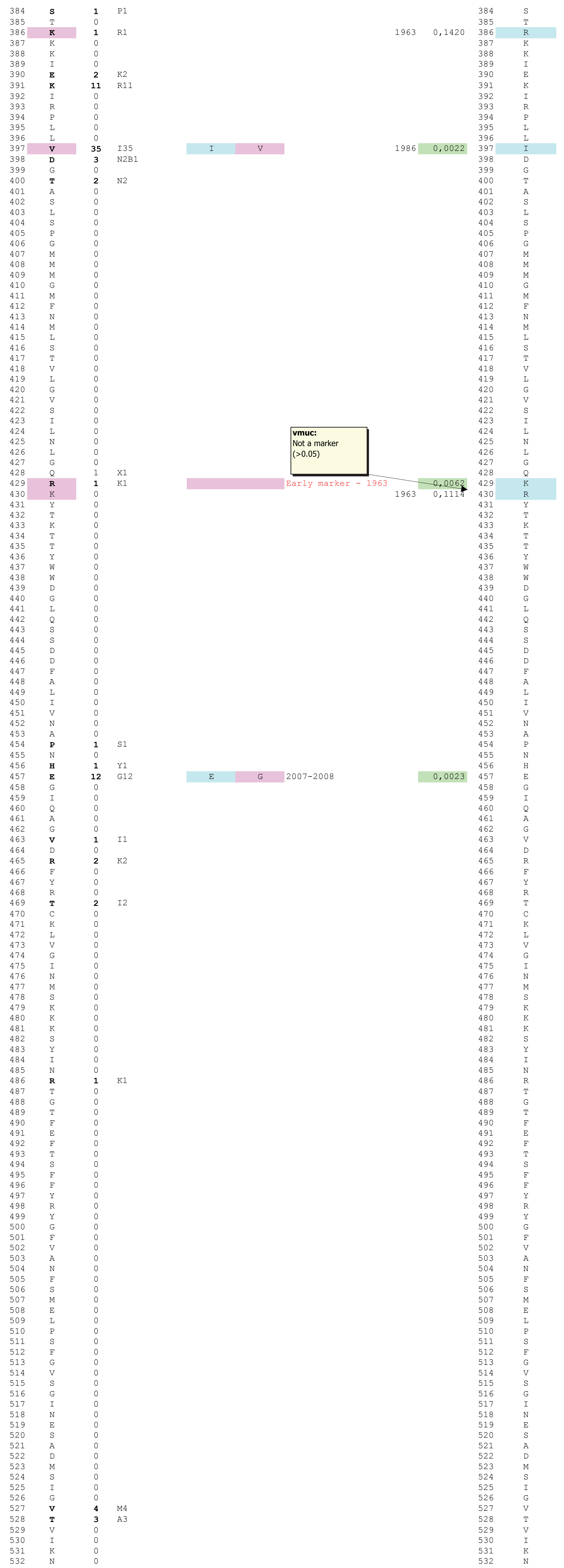

$48 \mathrm{R}=4260, \mathrm{~N}=1, \mathrm{Del}=3943, \mathrm{Q}=1, \mathrm{G}=2, \mathrm{~K}=1364, \mathrm{~T}=1, \mathrm{Xaa}=37$
$37 \mathrm{R}=85, \mathrm{~N}=8, \mathrm{Q}=113, \mathrm{E}=223, \mathrm{~K}=9140, \mathrm{M}=2, \mathrm{~T}=1, \mathrm{Xaa}=37$

$5 \mathrm{R}=10, \mathrm{~K}=9560, \mathrm{~T}=1, \mathrm{Xaa}=38$

$16 \mathrm{D}=2, \mathrm{Q}=1, \mathrm{E}=9448, \mathrm{G}=4, \mathrm{I}=10, \mathrm{~L}=1, \mathrm{~K}=23, \mathrm{M}=80, \mathrm{~V}=2, \mathrm{Xaa}=38$

$12 \mathrm{R}=201, \mathrm{~N}=25, \mathrm{E}=2, \mathrm{~K}=9341, \mathrm{~T}=2, \mathrm{Xaa}=38$

$5 \mathrm{R}=9563, \mathrm{I}=1, \mathrm{~K}=5, \mathrm{Xaa}=40$

列

$5 \mathrm{I}=5, \mathrm{~L}=9562, \mathrm{X}=2, \mathrm{~N}=1$

$29 \mathrm{R}=2, \mathrm{~N}=5, \mathrm{I}=9281, \mathrm{~L}=134, \mathrm{M}=77, \mathrm{~T}=47, \mathrm{~V}=21, \mathrm{Xaa}=42$

$39 \mathrm{~A}=1, \mathrm{~N}=28, \mathrm{D}=8996, \mathrm{E}=535, \mathrm{G}=4, \mathrm{Y}=4, \mathrm{Xaa}=41$

$10 \mathrm{D}=8, \mathrm{G}=9507, \mathrm{P}=1, \mathrm{~S}=51, \mathrm{Xaa}=42$
$11 \mathrm{~A}=79, \mathrm{~K}=1, \mathrm{P}=1, \mathrm{~T}=9487, \mathrm{Xaa}=41$

$11 \mathrm{~A}=19, \mathrm{~K}=1, \mathrm{P}=1, \mathrm{~T}=9487, \mathrm{Xaa}=41$
$\mathrm{~A}=9553, \mathrm{P}=1, \mathrm{~S}=1, \mathrm{~T}=1, \mathrm{~V}=13, \mathrm{Xaa}=40$

$6 \mathrm{~A}=9553, \mathrm{P}=1, \mathrm{~S}=1, \mathrm{~T}=1, \mathrm{~V}=13, \mathrm{x}$
$\mathrm{A}=26, \mathrm{P}=1, \mathrm{~S}=9541, \mathrm{Xaa}=41$

(2)

$\mathrm{S}=9562, \mathrm{~T}=2, \mathrm{Xaa}=42$

$4 \mathrm{De}=1, \mathrm{~L}=1, \mathrm{P}=9566, \mathrm{Xaa}=41$

$5 \mathrm{R}=1, \mathrm{Del}=1, \mathrm{~L}=1, \mathrm{M}=9562, \mathrm{~T}=1, \mathrm{~V}=2, \mathrm{Xaa}=41$

$5 \mathrm{R}=1, \mathrm{I}=3, \mathrm{~L}=2, \mathrm{M}=9561, \mathrm{Xaa}=42$

$4 \mathrm{G}=9566, \mathrm{~V}=1, \mathrm{Xaa}=42$

$4 \mathrm{I}=1, \mathrm{M}=9564, \mathrm{~T}=2, \mathrm{Xaa}=42$
$4 \mathrm{I}=1, \mathrm{~L}=1, \mathrm{~F}=9565, \mathrm{Xaa}=42$

$5 \mathrm{~N}=9559, \mathrm{D}=2, \mathrm{H}=1, \mathrm{~S}=2, \mathrm{~T}=3, \mathrm{Xaa}=42$

$\mathrm{I}=1, \mathrm{~L}=1, \mathrm{M}=1564, \mathrm{I}=1, \mathrm{~V}=1, \mathrm{Xaa}=4$

$4 \mathrm{R}=1, \mathrm{~S}=9568$, $\mathrm{xaa}=40$

$4 \mathrm{~S}=1, \mathrm{~T}=9568, \mathrm{Xaa}=40$
$4 \mathrm{I}=2, \mathrm{Y}=1, \mathrm{~V}=9567, \mathrm{Xaa}=39$

$4 \mathrm{C}=1, \mathrm{~L}=9566, \mathrm{~F}=1, \mathrm{~V}=1, \mathrm{Xaa}=40$

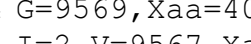

$11 \mathrm{~A}=42, \mathrm{Q}=1, \mathrm{~L}=2, \mathrm{~F}=1, \mathrm{P}=1, \mathrm{~S}=9503, \mathrm{~T}=19, \mathrm{Xaa}=40$

$14 \mathrm{I}=9440, \mathrm{~L}=1, \mathrm{~T}=1, \mathrm{~V}=127, \mathrm{Xaa}=40$

$4 \mathrm{~L}=9567, \mathrm{P}=1, \mathrm{~V}=1, \mathrm{Xaa}=40$

$\mathrm{N}=9565, \mathrm{I}=1, \mathrm{~K}=1, \mathrm{~S}=2, \mathrm{Xaa}=4$

$4 \mathrm{~A}=1, \mathrm{G}=9567, \mathrm{~W}=1, \times \mathrm{x} a=40$

$5 \mathrm{R}=1, \mathrm{Q}=9564, \mathrm{E}=1, \mathrm{H}=1, \mathrm{~L}=1, \mathrm{~K}=1, \mathrm{Xaa}=40$

$10 \mathrm{R}=60, \mathrm{~N}=3, \mathrm{Q}=6, \mathrm{~K}=9502, \mathrm{Xaa}=38$

$56 \mathrm{R}=8488, \mathrm{E}=8, \mathrm{G}=3, \mathrm{~K}=1070, \mathrm{~S}=1, \mathrm{~T}=1, \mathrm{Xa} a=38$

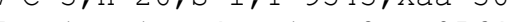

$13 \mathrm{R}=92, Q=14, \mathrm{~B}=1, \mathrm{~K}=9466, \mathrm{X} a \mathrm{a}=36, \mathrm{Xaa}=36$

$4 \mathrm{~A}=1, \mathrm{~N}=3, \mathrm{~K}=1, \mathrm{~T}=9568, \mathrm{Xaa}=36$

$8 \mathrm{~A}=5, \mathrm{I}=28, \mathrm{M}=3, \mathrm{P}=1, \mathrm{~S}=5, \mathrm{~T}=9530, \mathrm{Xaa}=37$
$5 \mathrm{C}=1, \mathrm{H}=2, \mathrm{~F}=8, \mathrm{Y}=9563, \mathrm{Xaa}=35$

L $=2, \mathrm{~W}=9572, \mathrm{Xaa}=35$

$\mathrm{R}=3, \mathrm{G}=1, \mathrm{~W}=9570, \mathrm{Xaa}=35$

$3 \mathrm{E}=1, \mathrm{G}=9575, \mathrm{Xaa}=33$

$3 \mathrm{~L}=9578$, $\mathrm{Xa} a=31$

$4 \mathrm{R}=1, \mathrm{Q}=9573, \mathrm{~L}=1, \mathrm{P}=2, \mathrm{xaa}=32$

$3 \mathrm{P}=1, \mathrm{~S}=9576, \mathrm{xa}=32$
$3 \mathrm{~F}=1, \mathrm{~S}=9575, \mathrm{xa}=33$

$4 \mathrm{~N}=2, \mathrm{D}=9573, \mathrm{E}=1, \mathrm{G}=2, \mathrm{Xaa}=31$

$4 \mathrm{~N}=3, \mathrm{D}=9575, \mathrm{xa} a=31$

$4 \mathrm{~A}=9570, \mathrm{G}=1, \mathrm{~T}=6, \mathrm{~V}=2, \mathrm{Xaa}=30$

$\mathrm{I}=1, \mathrm{~L}=9576, \mathrm{~F}=1, \mathrm{P}=1, \mathrm{Xaa}=30$

$13 \mathrm{G}=1, \mathrm{I}=14, \mathrm{~L}=74, \mathrm{M}=12, \mathrm{~V}=9479, \mathrm{Xaa}=29$

$\mathrm{N}=9577, \mathrm{D}=1, \mathrm{Y}=2, \mathrm{Xa} a=2$

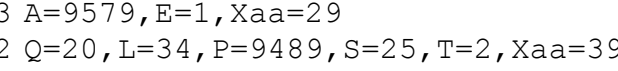

$16 \mathrm{R}=11, \mathrm{~N}=9, \mathrm{Q}=6, \mathrm{G}=9440, \mathrm{~L}=1, \mathrm{P}=1, \mathrm{Y}=103, \mathrm{Xa} a=38$

$9 \mathrm{~A}=1, \mathrm{D}=17, \mathrm{E}=9527, \mathrm{G}=22, \mathrm{~K}=4, \mathrm{Xaa}=38$

$5 \mathrm{E}=9, \mathrm{G}=9558, \mathrm{~S}=1, \mathrm{~V}=4, \mathrm{Xaa}=37$

$6 \mathrm{I}=9549, \mathrm{M}=1, \mathrm{~V}=23, \mathrm{Xaa}=36$

$3 \mathrm{~A}=9586, \mathrm{P}=2, \mathrm{~S}=1, \mathrm{Xaa}=20$

$\mathrm{R}=4, \mathrm{E}=1, \mathrm{G}=9585, \mathrm{xaa}=19$
$\mathrm{G}=2, \mathrm{G}=1, \mathrm{I}=36, \mathrm{M}=1, \mathrm{~V}=9550, \mathrm{xaa}=19$

$10 \mathrm{~A}=1, \mathrm{~N}=81, \mathrm{D}=9506, \mathrm{E}=1, \mathrm{~S}=1, \mathrm{Xaa}=19$

$4 \mathrm{R}=9568, \mathrm{~K}=21, \mathrm{~W}=1, \mathrm{Xaa}=19$

2 $\mathrm{F}=9590, \mathrm{~S}=1$, $\mathrm{Xaa}=18$

$2 \mathrm{~F}=1, \mathrm{Y}=9590, \mathrm{Xaa}=18$

政 $\mathrm{A}=7, \mathrm{~T}=9, \mathrm{~S}=2, \mathrm{~K}=4, \mathrm{M}=1, \mathrm{Xaa}=1$

$3 \mathrm{D}=1, \mathrm{C}=9587, \mathrm{G}=1, \mathrm{~S}=1, \mathrm{~W}=2, \mathrm{Xaa}=17$

$2 \mathrm{E}=1, \mathrm{~K}=9591, \mathrm{Xaa}=17$

$2 \mathrm{~L}=9589, \mathrm{M}=2, \mathrm{~V}=1, \mathrm{Xaa}=17$

$7 \mathrm{~A}=3, \mathrm{I}=34, \mathrm{~L}=8, \mathrm{~V}=9546, \mathrm{xaa}=18$

$2 \mathrm{G}=9592, \mathrm{xaa}=17$

$2 \mathrm{~N}=9588, \mathrm{D}=1, \mathrm{I}=2, \mathrm{Xaa}=17$

$\mathrm{L}=1, \mathrm{M}=9587, \mathrm{~T}=2, \mathrm{~V}=3, \mathrm{xa} \mathrm{xa}=16$

$3 \mathrm{R}=1, \mathrm{~N}=6, \mathrm{~S}=9583, \mathrm{~T}=3, \mathrm{xaa}=16$

$2 \mathrm{Q}=2, \mathrm{E}=1, \mathrm{~K}=9590, \mathrm{Xaa}=1$
$4 \mathrm{R}=21, \mathrm{~K}=9572, \mathrm{Xaa}=16$

$2 \mathrm{~N}=1, \mathrm{E}=1, \mathrm{~K}=9590, \mathrm{~T}=1, \mathrm{Xaa}=16$

$2 \mathrm{~N}=1, \mathrm{C}=1, \mathrm{~S}=1, \mathrm{Y}=9590, \mathrm{Xaa}=16$
$\mathrm{I}=9580, \mathrm{~L}=3, \mathrm{M}=8, \mathrm{~V}=1, \mathrm{Xaa}=17$

$2 \mathrm{~N}=9592, \mathrm{D}=1, \mathrm{Xaa}=16, \mathrm{X}$

R=9524, $\mathrm{Q}=1, \mathrm{G}=1, \mathrm{~L}=1, \mathrm{~K}=64, \mathrm{P}=1, \mathrm{Xaa}=17$

$2 \mathrm{~A}=2, \mathrm{R}=1, \mathrm{P}=1, \mathrm{I}=95$
$2 \mathrm{G}=9593, \mathrm{Xaa}=16$

$2 \mathrm{~T}=9593, \mathrm{Xaa}=16$

$3 \mathrm{C}=5, \mathrm{~L}=1, \mathrm{~F}=9586, \mathrm{Xaa}=17$

2 $\mathrm{D}=1, \mathrm{E}=9592, \mathrm{Xaa}=16$
$\mathrm{C}=2, \mathrm{~L}=4, \mathrm{~F}=9584, \mathrm{~S}=3, \mathrm{Xaa}=16$

$2 \mathrm{~T}=9593, \mathrm{Xaa}=16$

$2 \mathrm{~L}=1, \mathrm{~F}=9591, \mathrm{~S}=1, \mathrm{Xaa}=16$
$2 \mathrm{~L}=2, \mathrm{~F}=9590, \mathrm{Y}=1, \mathrm{Xaa}=16$

$\mathrm{N}=1, \mathrm{C}=3, \mathrm{H}=1, \mathrm{~F}=2, \mathrm{~S}=1, \mathrm{Y}=9585, \mathrm{Xaa}=16$

$2 \mathrm{R}=9588, \mathrm{C}=2, \mathrm{H}=2, \mathrm{P}=1, \mathrm{Xaa}=16$
$\mathrm{~N}=1, \mathrm{H}=4, \mathrm{~F}=1, \mathrm{Y}=9586, \mathrm{Xaa}=17$

$2 \mathrm{G}=9593, \mathrm{Xaa}=16$

$2 \mathrm{~F}=9593$, $\mathrm{Xaa}=16$

$3 \mathrm{I}=6, \mathrm{~V}=9587, \mathrm{Xaa}=16$
$3 \mathrm{~A}=9582, \mathrm{P}=2, \mathrm{~S}=9, \mathrm{Xaa}=16$

$2 \mathrm{~N}=9590, \mathrm{H}=1, \mathrm{P}=1, \mathrm{~S}=1, \mathrm{Xaa}=1$

$2 \mathrm{~S}=9592, \mathrm{~T}=1, \mathrm{Xaa}=16$

$2 \mathrm{I}=2, \mathrm{M}=9590, \mathrm{~T}=1, \mathrm{xaa}=16$

$2 \mathrm{D}=2, \mathrm{E}=9590, \mathrm{~K}=1, \mathrm{Xaa}=16$
$2 \mathrm{~L}=9592, \mathrm{~F}=1, \mathrm{Xaa}=16$

$2 \mathrm{~A}=1, \mathrm{P}=9592, \mathrm{Xaa}=16$

$3 \mathrm{~L}=9, \mathrm{~F}=9581, \mathrm{~S}=1, \mathrm{Y}=2, \mathrm{Xaa}=16$

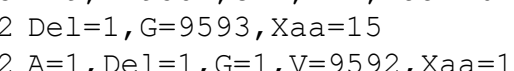

$2 \mathrm{~A}=1, \mathrm{Del}=1, \mathrm{G}=1, \mathrm{~V}=9592, \mathrm{Xaa}=14$

$8 \mathrm{~A}=26, \mathrm{Del}=1, \mathrm{P}=34, \mathrm{~S}=\mathrm{Xa} 33, \mathrm{Y}=$

$2 \mathrm{R}=2, \mathrm{De} l=1, \mathrm{G}=9593, \mathrm{Xaa}=13$
$16 \mathrm{~N}=1, \mathrm{I}=9407, \mathrm{~T}=15, \mathrm{~V}=173, \mathrm{Xaa}=13$
$1 \mathrm{~N}=9597, \mathrm{Y}=1, \mathrm{Xaa}=11$

2 $\mathrm{D}=1, \mathrm{E}=9596, \mathrm{G}=1, \mathrm{Xaa}=11$

$\mathrm{A}=9595, \mathrm{~S}=2, \mathrm{~T}=1, \mathrm{Xaa}=11$

$1 \mathrm{D}=9598, \mathrm{Xa}=11, \mathrm{X} a \mathrm{I}=13, \mathrm{X}=2, \mathrm{M}=9581, \mathrm{~T}=2, \mathrm{Xaa}=11$
$3 \mathrm{I}=13, \mathrm{~N}$

$6 \mathrm{I}=9549, \mathrm{M}=7, \mathrm{~T}=3, \mathrm{~V}=39, \mathrm{X} a \mathrm{a}=11$

$1 \mathrm{G}=9598$, Xaa $=1$

$18 \mathrm{~A}=9, \mathrm{I}=192, \mathrm{~L}=8, \mathrm{~S}=1, \mathrm{~V}=9388, \mathrm{Xaa}=11$

$4 \mathrm{~A}=23, \mathrm{I}=1, \mathrm{P}=1, \mathrm{~S}=1, \mathrm{~T}=9572, \mathrm{xa} a=1$

$3 \mathrm{I}=2, \mathrm{~L}=3, \mathrm{M}=5, \mathrm{~V}=9588, \mathrm{Xaa}=1$

$\mathrm{R}=33, \mathrm{E}=1, \mathrm{~K}=9565, \mathrm{Xaa}=10$

$\mathrm{N}=9595, \mathrm{D}=1, \mathrm{~S}=2$, 


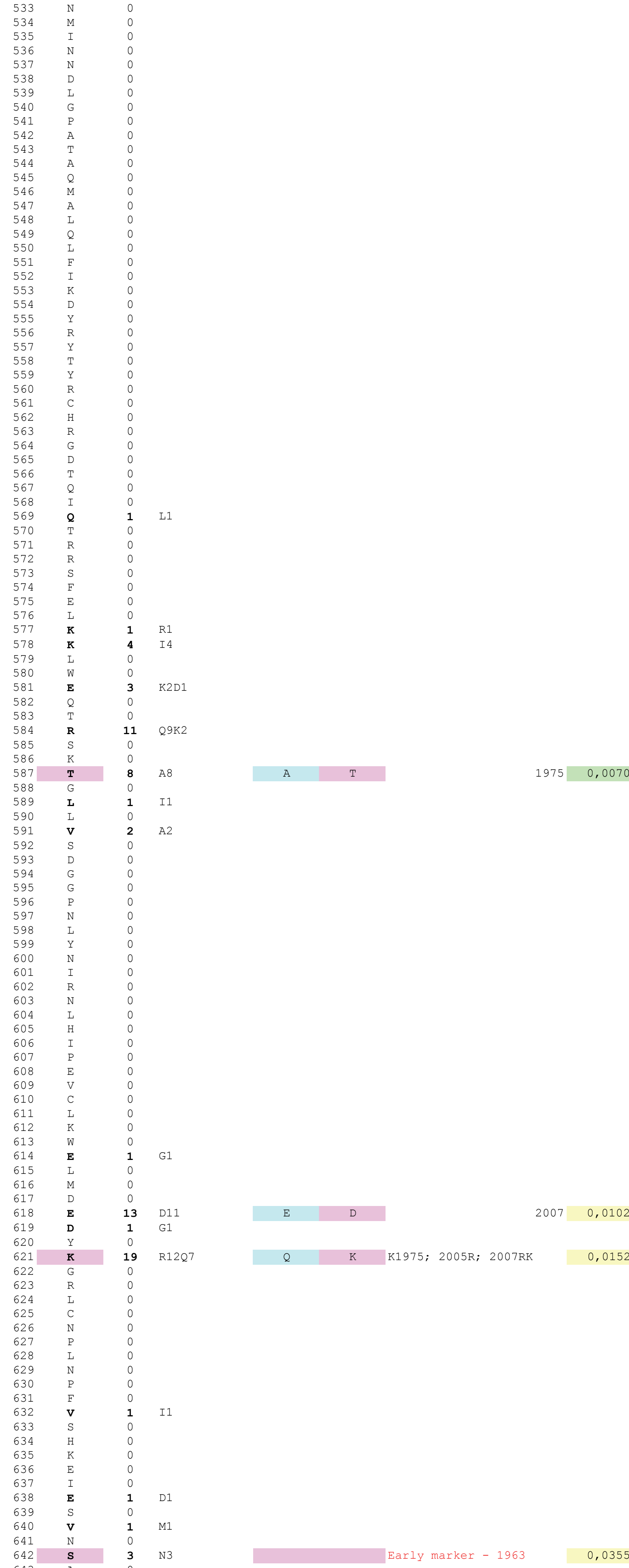


$1 \mathrm{~A}=1, \mathrm{E}=9604, \mathrm{~K}=2, \mathrm{~V}=1$

$1 \mathrm{~N}=1, \mathrm{D}=9602, \mathrm{E}=1, \mathrm{G}=1, \mathrm{Y}=2, \mathrm{Xaa}=1$

$1 \mathrm{~A}=1, \mathrm{D}=2, \mathrm{E}=9604, \mathrm{Xaa}=1$

$5 \mathrm{R}=19, \mathrm{Q}=9567, \mathrm{H}=7, \mathrm{~L}=3, \mathrm{~K}=12$

2 $\mathrm{I}=4, \mathrm{~L}=1, \mathrm{M}=9596, \mathrm{~V}=7$

$\mathrm{D}=1, \mathrm{C}=1, \mathrm{H}=1, \mathrm{Y}=9605$

$4 \mathrm{R}=32, \mathrm{~N}=2, \mathrm{E}=2, \mathrm{~K}=9569, \mathrm{Xaa}=3$

$\mathrm{O}=9608$

$0 \mathrm{C}=9605, \mathrm{Y}=3$

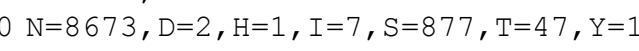

$2 \mathrm{Q}=1, \mathrm{H}=1, \mathrm{I}=11, \mathrm{~L}=9594, \mathrm{~V}=$

$1 \mathrm{I}=1, \mathrm{~L}=2, \mathrm{~F}=9604, \mathrm{~S}=1$

$2 \mathrm{R}=4, \mathrm{~N}=2, \mathrm{E}=3, \mathrm{~K}=9595, \mathrm{~T}=4$

$0 \mathrm{~L}=1, \mathrm{~F}=9606, \mathrm{Y}=1$

$\mathrm{L}=1, \mathrm{~F}=9606, \mathrm{~S}=$

P $\mathrm{P}=9608$

O $R=1, S=9607$

$1 \mathrm{C}=2, \mathrm{G}=4, \mathrm{~S}=9602$

$\mathrm{L}=1, \mathrm{P}=1, \mathrm{~S}=9605, \mathrm{~T}=1$

$\mathrm{C}=1, \mathrm{Y}=9607$

$1 \mathrm{R}=9600, \mathrm{G}=1, \mathrm{~K}=7$

$\mathrm{R}=96008$
$\mathrm{P}=9608$

$3 \mathrm{I}=32, \mathrm{~V}=9576$

$0 \mathrm{R}=1, \mathrm{G}=9607$

$1 \mathrm{I}=9604, \mathrm{~L}=1, \mathrm{~V}=3$

$\mathrm{C}=1, \mathrm{~S}=9605, \mathrm{Y}=2$

$1 \mathrm{R}=1, \mathrm{I}=1, \mathrm{~K}=1, \mathrm{M}=9604, \mathrm{~V}=1$

$10 \mathrm{~A}=6, \mathrm{G}=32, \mathrm{~L}=1, \mathrm{M}=60, \mathrm{~V}=9509$

$1 \mathrm{D}=5, \mathrm{Q}=1, \mathrm{E}=9600, \mathrm{G}=2$

$2 \mathrm{~A}=9588, \mathrm{~S}=20$

$5 \mathrm{~A}=5, \mathrm{I}=45, \mathrm{~L}=5, \mathrm{M}=120, \mathrm{~V}=9433$

$2 \mathrm{~F}=12, \mathrm{~S}=9594, \mathrm{Y}=1, \mathrm{Xaa}=1$

$1 \mathrm{R}=9604, \mathrm{~K}=2, \mathrm{~S}=1, \mathrm{~T}=1$

$2 \mathrm{R}=9595, \mathrm{Q}=10, \mathrm{~S}=1, \mathrm{P}=1, \mathrm{X}=1$

$\mathrm{I}=904, \mathrm{~T}=2, \mathrm{Xaa}, \mathrm{P}=1, \mathrm{Xaa}=1$

$\mathrm{N}=1, \mathrm{D}=9604, \mathrm{Y}=2$

$1 \mathrm{~A}=9600, \mathrm{P}=1, \mathrm{~S}=4, \mathrm{~T}=1, \mathrm{~V}=1$

$0 \mathrm{R}=9606, \mathrm{P}=1$

$8 \mathrm{I}=9531, \mathrm{~L}=2, \mathrm{~T}=38, \mathrm{~V}=36$

$1 \mathrm{~N}=1, \mathrm{D}=9601, \mathrm{E}=2, \mathrm{G}=1, \mathrm{~V}=2$

$1 \mathrm{~N}=1, \mathrm{D}=9601, \mathrm{E}=2, \mathrm{G}=1, \mathrm{~V}=\mathrm{L}$
$2 \mathrm{~L}=3, \mathrm{~F}=9595, \mathrm{~S}=7, \mathrm{~V}=2$

$1 \mathrm{D}=4, \mathrm{Q}=1, \mathrm{E}=9598, \mathrm{G}=1, \mathrm{H}=1, \mathrm{~K}=2$

$1 \mathrm{~A}=5, \mathrm{P}=1, \mathrm{~S}=9600, \mathrm{~T}=1$

$1 \mathrm{R}=4, \mathrm{E}=6, \mathrm{G}=9597$

$1 \mathrm{R}=9597, \mathrm{Q}=3, \mathrm{~K}=5, \mathrm{~T}=1, \mathrm{~W}=1$

$5 \mathrm{I}=9565, \mathrm{M}=7, \mathrm{~T}=6, \mathrm{~V}=29$

$8 \mathrm{R}=42, \mathrm{~N}=12, \mathrm{Del}=1, \mathrm{Q}=1, \mathrm{E}=12, \mathrm{~K}=9536, \mathrm{M}=1, \mathrm{~T}=1, \mathrm{Xaa}=1$

$6 \mathrm{R}=47, \mathrm{~N}=3, \mathrm{Q}=5, \mathrm{E}=4, \mathrm{~L}=1, \mathrm{~K}=9545, \mathrm{~T}=2$

$6 \mathrm{~A}=3, \mathrm{D}=16, \mathrm{Q}=8, \mathrm{E}=9552, \mathrm{G}=21, \mathrm{I}=1, \mathrm{~K}=5, \mathrm{~V}=1$

$8 \mathrm{D}=39, \mathrm{E}=9533, \mathrm{G}=7, \mathrm{~K}=26, \mathrm{~S}=1, \mathrm{~V}=1$

$3 \mathrm{C}=2, \mathrm{~L}=13, \mathrm{~F}=9579, \mathrm{~S}=1, \mathrm{Y}=11, \mathrm{~V}=1$

$15 \mathrm{~A}=9435, \mathrm{~N}=2, \mathrm{G}=2, \mathrm{~S}=33, \mathrm{~T}=123, \mathrm{~V}=12$

$2 \mathrm{D}=1, \mathrm{E}=9593, \mathrm{~K}=12, \mathrm{Xaa}=1$

$1 \mathrm{D}=9596, \mathrm{M}=1, \mathrm{~T}=3, \mathrm{~V}=6, \mathrm{Xaa}=1$

$32 \mathrm{~A}=4, \mathrm{I}=141, \mathrm{~L}=142, \mathrm{~K}=1, \mathrm{M}=9221, \mathrm{~T}=69, \mathrm{~V}=28, \mathrm{Xaa}=1$

$10 \mathrm{R}=43, \mathrm{Q}=1, \mathrm{E}=56, \mathrm{G}=1, \mathrm{~K}=9501, \mathrm{M}=1, \mathrm{~T}=3, \mathrm{Xaa}=1$

$11 \mathrm{I}=9465, \mathrm{~F}=1, \mathrm{~T}=140, \mathrm{~V}=1$

$\mathrm{O} R=2, \mathrm{C}=9604, \mathrm{~S}=1$

$6 \mathrm{~A}=1, \mathrm{C}=9, \mathrm{~F}=20, \mathrm{P}=2, \mathrm{~S}=9555, \mathrm{~T}=4, \mathrm{Y}=15, \mathrm{~V}=1$

$2 \mathrm{~A}=2, \mathrm{I}=1, \mathrm{P}=3, \mathrm{~S}=4, \mathrm{~T}=9596, \mathrm{Xaa}=1$

$1 \mathrm{I}=9602, \mathrm{~L}=1, \mathrm{M}=1, \mathrm{~T}=2, \mathrm{Xaa}=1$

$2 \mathrm{~A}=1, \mathrm{E}=9594, \mathrm{G}=3, \mathrm{~K}=9$

$5 \mathrm{~A}=3, \mathrm{D}=12, \mathrm{E}=9565, \mathrm{G}=13, \mathrm{~K}=13, \mathrm{~V}=1$

$6 \mathrm{H}=4, \mathrm{I}=14, \mathrm{~N}=9552, \mathrm{~F}=21, \mathrm{P}=4, \mathrm{~V}=12$

$10 \mathrm{R}=9505, \mathrm{Q}=1, \mathrm{E}=2, \mathrm{G}=65, \mathrm{~K}=34$

$1 \mathrm{R}=9600, \mathrm{Q}=4, \mathrm{P}=3$

$32 \mathrm{R}=48, \mathrm{Q}=9159, \mathrm{E}=1, \mathrm{H}=29, \mathrm{~L}=3, \mathrm{~K}=2, \mathrm{P}=361, \mathrm{~S}=2, \mathrm{Xaa}=1$

$28 \mathrm{R}=148, \mathrm{~N}=79, \mathrm{Q}=27, \mathrm{E}=6, \mathrm{G}=49, \mathrm{I}=2, \mathrm{~K}=9282, \mathrm{M}=1, \mathrm{~S}=1, \mathrm{~T}=8$

$83 \mathrm{R}=20, \mathrm{~N}=1, \mathrm{O}=489, \mathrm{~K}=45, \mathrm{~S}=2, \mathrm{~W}=12, \mathrm{Y}=1$ 
Pld 



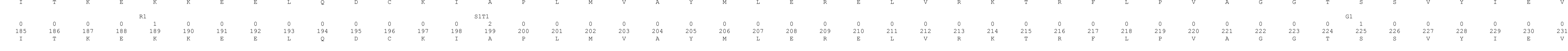





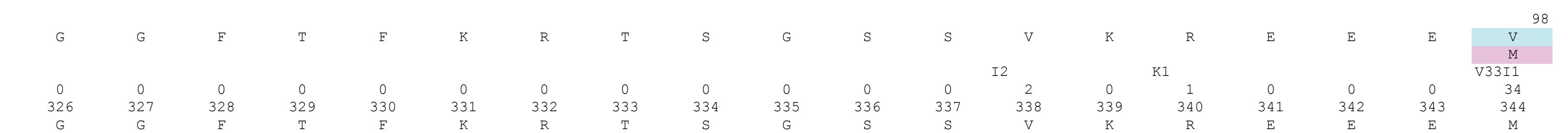




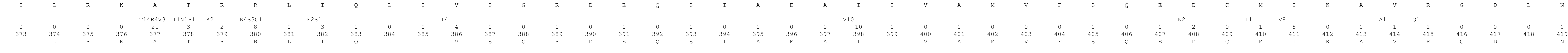




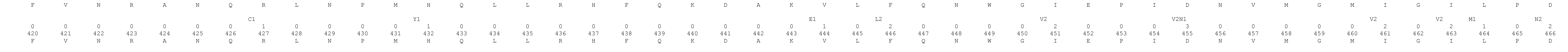


40

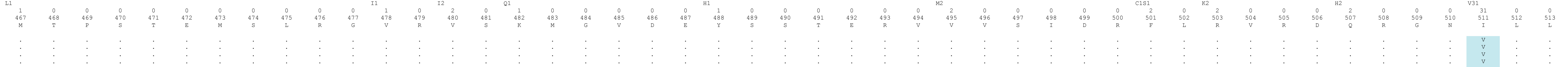




$$
11
$$




$$
\mid
$$


- 


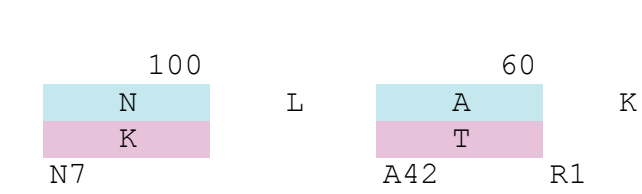

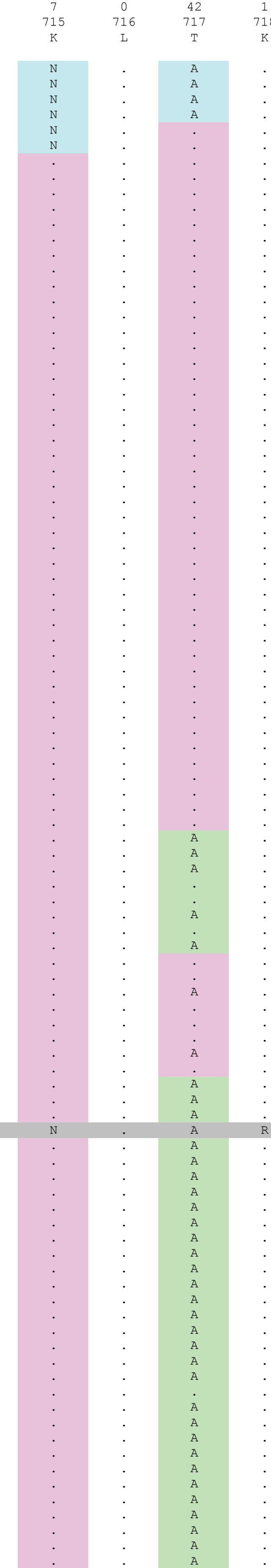




\begin{tabular}{|c|c|c|c|c|c|c|c|c|c|c|c|}
\hline $\mathrm{T}$ & A & $\mathrm{T}$ & K & $\mathrm{R}$ & I & $\mathrm{R}$ & M & A & I & $\mathrm{N}$ & \\
\hline & & & & & & & & \multicolumn{4}{|c|}{ S2 } \\
\hline 0 & 0 & 1 & 0 & 0 & 0 & 0 & 0 & 0 & 0 & 2 & 97 \\
\hline 749 & 750 & 751 & 752 & 753 & 754 & 755 & 756 & 757 & 758 & 759 & 760 \\
\hline $\mathrm{T}$ & A & $\mathrm{T}$ & K & $\mathrm{R}$ & I & $\mathrm{R}$ & M & A & I & $\mathrm{N}$ & $\mathrm{Y}$ \\
\hline
\end{tabular}




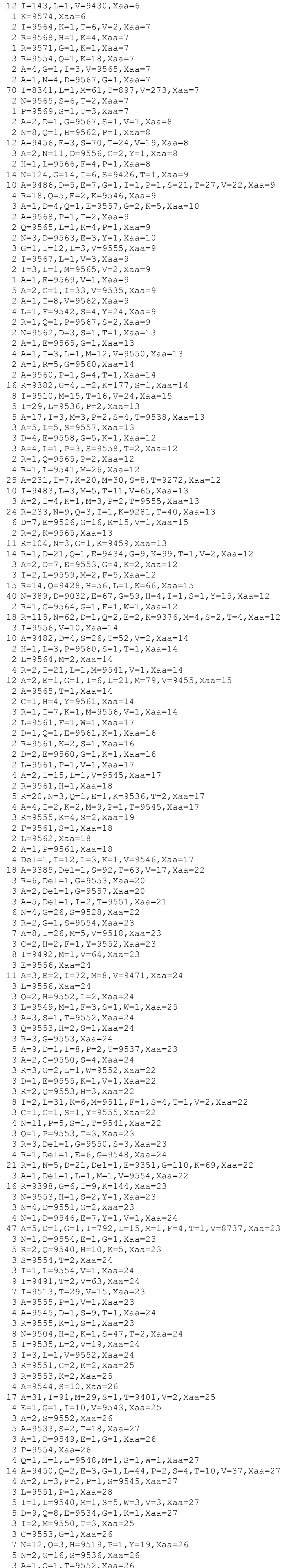


作

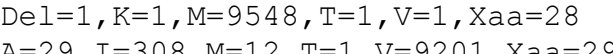

$\mathrm{A}=29, \mathrm{I}=308, \mathrm{M}=12, \mathrm{~T}=1, \mathrm{~V}=9201, \mathrm{xaa}=29$

$5 \mathrm{~A}=1, \mathrm{~N}=4, \mathrm{D}=9540, \mathrm{E}=4, \mathrm{G}=2, \mathrm{Xaa}=28$
$8 \mathrm{R}=1, \mathrm{I}=9496, \mathrm{~L}=2, \mathrm{~T}=2, \mathrm{~V}=52, \mathrm{Xaa}=27$

$\mathrm{I}=5, \mathrm{~L}=9535, \mathrm{~F}=9, \mathrm{Xaa}=31$

$19 \mathrm{R}=9348, \mathrm{Q}=4, \mathrm{~L}=4, \mathrm{~K}=183, \mathrm{M}=7, \mathrm{~T}=4, \mathrm{Xaa}=30$

$4 \mathrm{~N}=9546, \mathrm{H}=1, \mathrm{I}=2, \mathrm{~S}=3, \mathrm{Y}=1, \mathrm{Xa}=27$

$4 \mathrm{~L}=1, \mathrm{P}=9539, \mathrm{~S}=12, \mathrm{~T}=1, \mathrm{Xaa}=27$

$7 \mathrm{~A}=1, \mathrm{~N}=1, \mathrm{H}=1, \mathrm{I}=14, \mathrm{M}=1, \mathrm{P}=2, \mathrm{~S}=16, \mathrm{~T}=9516, \mathrm{Xaa}=28$

$3 \mathrm{E}=9551, \mathrm{~K}=1, \mathrm{~V}=1, \mathrm{Xaa}=27$

$3 \mathrm{R}=2, \mathrm{Q}=9550, \mathrm{P}=1, \mathrm{Xaa}=27$

$3 \mathrm{~A}=9553, \mathrm{Xaa}=27$

$\begin{array}{rl}4 & \mathrm{E}=1, \mathrm{G}=\mathrm{X}, \mathrm{I}=4, \mathrm{M}=1, \mathrm{~V}=9546, \mathrm{Xaa}=27 \\ 20 \mathrm{~A}=1, \mathrm{~N}=73, \mathrm{D}=9361, \mathrm{E}=67, \mathrm{G}=49, \mathrm{Y}=2, \mathrm{Xaa}=27\end{array}$

$3 \mathrm{I}=9549, \mathrm{M}=3, \mathrm{~F}=1, \mathrm{Xaa}=27$
$\mathrm{C}=9553, \mathrm{~F}=1, \mathrm{Xa}=26$

$8 \mathrm{R}=62, \mathrm{~K}=9491, \mathrm{M}=1, \mathrm{Xaa}=26$

$3 \mathrm{~A}=9549, \mathrm{G}=1, \mathrm{~S}=1, \mathrm{~T}=1, \mathrm{~V}=2, \mathrm{Xaa}=26$

$4 \mathrm{~A}=9539, \mathrm{~S}=14, \mathrm{Xaa}=27$

$24 \mathrm{I}=114, \mathrm{~L}=151, \mathrm{M}=9284, \mathrm{~V}=5, \mathrm{xaa}=26$

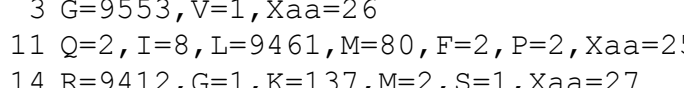

$4 \mathrm{I}=9541, \mathrm{~T}=2, \mathrm{~V}=12, \mathrm{Xaa}=25$

$4 \mathrm{R}=3, \mathrm{~N}=6, \mathrm{~F}=2, \mathrm{~S}=9543, \mathrm{~T}=1, \mathrm{Xaa}=25$

$3 \mathrm{~L}=2, \mathrm{P}=2, \mathrm{~S}=9551, \mathrm{Xaa}=25$
$3 \mathrm{~F}=2, \mathrm{~S}=9551, \mathrm{~T}=1, \mathrm{Xaa}=26$

N $=1, Q=1, \mathrm{C}=2, \mathrm{~S}=9548, \mathrm{~T}=2, \mathrm{Xaa}=2$

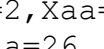

$3 \mathrm{R}=1, \mathrm{C}=1, \mathrm{G}=9552, \mathrm{Xaa}=26$

$6 \mathrm{R}=4, \mathrm{~N}=3, \mathrm{E}=4, \mathrm{G}=9524, \mathrm{~S}=19, \mathrm{Xaa}=26$

$3 \mathrm{~L}=3, \mathrm{~F}=9550, \mathrm{Y}=2, \mathrm{Xaa}=25$

xaa $=25$

R=1, $=1, \mathrm{~B}=1, \mathrm{~K}=9545, \mathrm{M}=7, \mathrm{X}$

$6 \mathrm{R}=9522, \mathrm{Q}=1, \mathrm{G}=2, \mathrm{I}=2, \mathrm{~K}=28, \mathrm{X}=2 \mathrm{a}=25$

$3 \mathrm{~A}=1, \mathrm{~T}=9553, \mathrm{Xaa}=26$

$11 \mathrm{R}=11, \mathrm{~N}=12, \mathrm{G}=2, \mathrm{~K}=57, \mathrm{~S}=9472, \mathrm{Xaa}=2$

$\mathrm{B} A=2, \mathrm{H}=1, \mathrm{~L}=7, \mathrm{C}$

$=9504, T=23, V=1, X a a=26$

$3 \mathrm{~A}=1, \mathrm{~F}=1, \mathrm{~S}=$

$=7, \mathrm{P}=15, \mathrm{P}=1, \mathrm{~S}=9504, \mathrm{I}=23, \mathrm{~V}=1, \mathrm{X} \mathrm{a}=26 \mathrm{~V}$

$5 \mathrm{~A}=5, \mathrm{R}=46, \mathrm{~N}=14, \mathrm{Q}=8, \mathrm{E}=2, \mathrm{G}=89, \mathrm{~K}=8637, \mathrm{M}=20, \mathrm{~T}=731, \mathrm{~V}=2$, $\mathrm{Xaa}=26$

$7 \mathrm{D}=7, \mathrm{Q}=1, \mathrm{E}=9512, \mathrm{G}=19, \mathrm{~K}=15, \mathrm{Xaa}=26$

$6 \mathrm{R}=3, \mathrm{D}=16, \mathrm{De}=1, \mathrm{E}=9528, \mathrm{G}=7, \mathrm{~V}=1, \mathrm{Xaa}=24$
$\mathrm{~A}=1, \mathrm{De}=1=1, \mathrm{E}=2, \mathrm{I}=3, \mathrm{~L}=17, \mathrm{M}=166, \mathrm{~V}=9365, \mathrm{Xaa}=25$

3 $\mathrm{Del}=1, \mathrm{I}=1, \mathrm{~L}=9549, \mathrm{~F}=3$, $\mathrm{Xaa}=26$

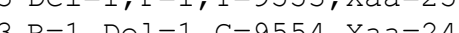

$3 \mathrm{~N}=9549, \mathrm{Q}=1, \mathrm{H}=1, \mathrm{~S}=4, \mathrm{Xaa}=25$

$3 \mathrm{~A}=1, \mathrm{~L}=9551, \mathrm{P}=4, \mathrm{Xaa}=24$

$3 \mathrm{Q}=9554, \mathrm{H}=2, \mathrm{~L}=1, \mathrm{Xaa}=23$

$6 \mathrm{~A}=29, \mathrm{R}=1, \mathrm{~K}=1, \mathrm{~T}=9526, \mathrm{xaa}=23$

$\begin{array}{rl}3 & \mathrm{~L}=9552, \mathrm{M}=1, \mathrm{~F}=3, \mathrm{~S}=1, \mathrm{Xaa}=23 \\ 14 \mathrm{R}=144, \mathrm{~N}=1, \mathrm{~F}=3, \mathrm{~K}=9409, \mathrm{X} a=23\end{array}$

$20 \mathrm{I}=9357, \mathrm{~L}=50, \mathrm{~K}=1, \mathrm{M}=61, \mathrm{~V}=88$, Xaa $=23$

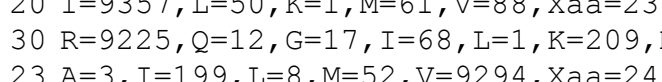

$23 \mathrm{~A}=3, \mathrm{I}=199, \mathrm{~L}=8, \mathrm{M}=52, \mathrm{~V}=9294, \mathrm{Xaa}=24$

$15 \mathrm{~A}=42, \mathrm{D}=4, \mathrm{Q}=4, \mathrm{E}=9442, \mathrm{G}=3, \mathrm{I}=2, \mathrm{~K}=9, \mathrm{X}=26, \mathrm{~V}=25, \mathrm{Xaa}=23$

$3 \mathrm{E}=1, \mathrm{G}=9555, \mathrm{Xaa}=24$

$6 \mathrm{C}=9, \mathrm{H}=18, \mathrm{~F}=2, \mathrm{~S}=1, \mathrm{Y}=9528, \mathrm{Xaa}=22$

$2 \mathrm{E}=9559, \mathrm{Xaa}=2, \mathrm{~s}$

$3 \mathrm{Q}=1, \mathrm{E}=9557, \mathrm{G}=1, \mathrm{Xaa}=2$

$\mathrm{A}$
$\mathrm{L}=20, \mathrm{~F}=9539, \mathrm{X} a \mathrm{a}=21$
$\mathrm{~A}=2, \mathrm{I}=3, \mathrm{P}=1, \mathrm{~T}=9554, \mathrm{xaa}=20$

$1 \mathrm{I}=79, \mathrm{~L}=11, \mathrm{M}=9461, \mathrm{~T}=1, \mathrm{~V}=8, \mathrm{Xaa}=20$

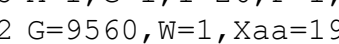

$49 \mathrm{R}=8778, \mathrm{Q}=639, \mathrm{G}=2, \mathrm{~L}=1, \mathrm{~K}=139, \mathrm{P}=1, \mathrm{Xaa}=20$

$15 \mathrm{R}=9422, \mathrm{G}=6, \mathrm{~K}=107, \mathrm{M}=4, \mathrm{~S}=20, \mathrm{~T}=1, \mathrm{~W}=2, \mathrm{Xa} a=18$

2 $\mathrm{A}=9559, \mathrm{G}=1, \mathrm{P}=1, \mathrm{~S}=1, \mathrm{x}$
$2 \mathrm{~A}=1, \mathrm{~T}=9561, \mathrm{Xaa}=18$

$\mathrm{A}=9561, \mathrm{~T}=1, \mathrm{Xaa}=18$

II $=57, \mathrm{~L}=9461, \mathrm{M}=20, \mathrm{~F}=15, \mathrm{~V}=8, \mathrm{Xaa}=19$
$\mathrm{R}=9551, \mathrm{E}=1, \mathrm{G}=1, \mathrm{~K}=4, \mathrm{M}=3, \mathrm{~W}=1$, $\mathrm{xaa}=19$

$3 \mathrm{R}=9551, \mathrm{E}=1, \mathrm{G}=1, \mathrm{~K}=4, \mathrm{M}=\mathrm{M}, \mathrm{M}$
$3 \mathrm{R}=2, \mathrm{E}=1, \mathrm{~K}=9558, \mathrm{Xaa}=19$

$6 \mathrm{~A}=9527, \mathrm{E}=1, \mathrm{G}=1, \mathrm{~S}=32, \mathrm{Xaa}=19$

$3 \mathrm{~A}=1, \mathrm{I}=2, \mathrm{P}=2, \mathrm{~S}=1, \mathrm{~T}=9555, \mathrm{Xaa}=19$

$\mathrm{R}=9541, \mathrm{~K}=1, \mathrm{~K}=17, \mathrm{M}=1, \mathrm{Xaa}=20$

$9 \mathrm{~L}=9482, \mathrm{M}=78, \mathrm{xaa}=20$

$I=9358, T=1, V=201, \mathrm{Xaa}=20$

$3 \mathrm{R}=1, \mathrm{Q}=9553, \mathrm{H}=3, \mathrm{~K}=1, \mathrm{P}=1$,
$3 \mathrm{I}=1, \mathrm{~L}=9553, \mathrm{M}=3, \mathrm{Xaa}=23$

$4 \mathrm{I}=9548, \mathrm{~L}=3, \mathrm{M}=1, \mathrm{~F}=1, \mathrm{~V}=6, \mathrm{Xaa}=21$

$4 \mathrm{~A}=5, \mathrm{G}=1, \mathrm{I}=6, \mathrm{I}=1, \mathrm{~V}=9545, \mathrm{Xa} a=22$

$7 \mathrm{~N}=2, \mathrm{C}=6, \mathrm{G}=31, \mathrm{~S}=\mathrm{y}$

$29 \mathrm{R}=9147, \mathrm{G}=7, \mathrm{~K}=401, \mathrm{~S}=2, \mathrm{Xaa}=23$

$29 \mathrm{R}=9147, \mathrm{G}=7, \mathrm{~K}=401, \mathrm{~S}=2, \mathrm{Xaa}=23$
$38 \mathrm{~A}=1, \mathrm{~N}=616, \mathrm{D}=8933, \mathrm{E}=4, \mathrm{G}=2, \mathrm{H}=1, \mathrm{I}=1, \mathrm{Xaa}=22$

7 $\mathrm{D}=19, \mathrm{Q}=10, \mathrm{E}=9522, \mathrm{G}=5, \mathrm{~K}=1, \mathrm{H}=1, \mathrm{Xaa}=22$

$3 \mathrm{Q}=9552, \mathrm{E}=2, \mathrm{H}=4, \mathrm{Xaa}=2$

$3 \mathrm{~A}=8, \mathrm{~L}=1, \mathrm{~S}=9549, \mathrm{Xaa}=22$

政

$=4, K=1, X a a=2$

$\mathrm{A}=9505, \mathrm{R}=1, \mathrm{G}=1, \mathrm{~S}=1, \mathrm{~T}=6, \mathrm{~V}=44, \mathrm{Xaa}=22$

$4 \mathrm{I}=9445, \mathrm{~L}=10, \mathrm{M}=22, \mathrm{~T}=47, \mathrm{~V}=3$
$\mathrm{I}=9554, \mathrm{M}=1, \mathrm{~T}=2, \mathrm{~V}=1, \mathrm{Xaa}=22$

, $533, \mathrm{xa}=22$

I

$\mathrm{I}=5, \mathrm{~L}=4, \mathrm{M}=9547, \mathrm{~V}=1, \mathrm{Xaa}=23$
$\mathrm{~A}=1, \mathrm{I}=3, \mathrm{~L}=1, \mathrm{~V}=9552, \mathrm{Xaa}=23$

$5 \mathrm{~L}=19, \mathrm{~F}=9536, \mathrm{~S}=1$, Xaa $=24$

$4 \mathrm{P}=8, \mathrm{~S}=9543, \mathrm{~T}=5, \mathrm{Xa} a=24$

$3 \mathrm{Q}=9553, \mathrm{~L}=1, \mathrm{P}=1, \mathrm{Xa \textrm {a } = 2 5}$

$3 \mathrm{D}=2, \mathrm{E}=9551, \mathrm{G}=2, \mathrm{Xaa}=25$
$9 \mathrm{~N}=5, \mathrm{D}=9485, \mathrm{E}=65, \mathrm{Xaa}=25$

$8 \mathrm{R}=31, \mathrm{C}=9503, \mathrm{G}=1, \mathrm{~S}=14, \mathrm{Y}=5$, Xaa $=26$

$\mathrm{I}=1, \mathrm{~L}=2, \mathrm{M}=9542, \mathrm{~T}=1, \mathrm{~V}=9, \mathrm{Xaa}=25$

$5 \mathrm{I}=9409, \mathrm{~L}=2, \mathrm{M}=26, \mathrm{~T}=1, \mathrm{~V}=117, \mathrm{Xaa}=25$

$\mathrm{R}=40, \mathrm{~K}=9511, \mathrm{~T}=3, \mathrm{Xad}=26$

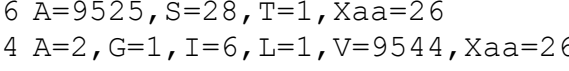

$3 \mathrm{R}=9553, \mathrm{Xaa}=27$

$4 \mathrm{D}=3, \mathrm{G}=9542, \mathrm{~S}=8, \mathrm{Xaa}=27$

$3 \mathrm{D}=9553, \mathrm{Xaa}=27$

$4 \mathrm{~N}=9548, \mathrm{E}=1, \mathrm{I}=2, \mathrm{~S}=1, \mathrm{~T}=1, \mathrm{Xaa}=2$

$\mathrm{L}=1, \mathrm{~F}=9546, \mathrm{~S}=1, \mathrm{Y}=1, \mathrm{~V}=3, \mathrm{Xaa}=28$
$\mathrm{~A}=1, \mathrm{I}=23, \mathrm{~L}=2, \mathrm{~F}=1, \mathrm{~V}=9526, \mathrm{Xaa}=27$

$3 \mathrm{~N}=9548, \mathrm{D}=2, \mathrm{I}=2, \mathrm{Xaa}=28$
$3 \mathrm{R}=9551, \mathrm{~K}=1, \mathrm{~S}=1, \mathrm{Xaa}=27$

$3 \mathrm{R}=9551, \mathrm{~K}=1, \mathrm{~S}=1, \mathrm{Xaa}=27$

$3 \mathrm{~N}=9550, \mathrm{H}=1, \mathrm{I}=1, \mathrm{~K}=1, \mathrm{~T}=1, \mathrm{Xaa}=26$

$4 \mathrm{R}=9548, \mathrm{I}=2, \mathrm{~L}=2, \mathrm{~K}=2, \mathrm{xaa}=2$

$3 \mathrm{I}=1, \mathrm{~L}=9551, \mathrm{~F}=1, \mathrm{~S}=1, \mathrm{Xaa}=2$

$3 \mathrm{~N}=9551, \mathrm{~F}=1, \mathrm{~S}=1, \mathrm{Xaa}=2$

$11 \mathrm{P}=9453, \mathrm{~T}=100, \mathrm{Xaa}=27$

$3 \mathrm{~L}=1, \mathrm{M}=9552, \mathrm{Xaa}=27$
$5 \mathrm{R}=1, \mathrm{H}=9539, \mathrm{P}=2, \mathrm{Y}=11$

Q $Q=9551, \mathrm{H}=2, \mathrm{x}=2, \mathrm{a}=27, \mathrm{xaa}=27$

$4 \mathrm{~L}=9543, \mathrm{~F}=7, \mathrm{P}=2, \mathrm{Xaa}=2$

$3 \mathrm{Q}=1, \mathrm{~L}=9549, \mathrm{M}=2, \mathrm{~V}=1, \mathrm{xaa}=27$

$\mathrm{N}=1, \mathrm{H}=9549, \mathrm{~L}=1, \mathrm{M}=1, \mathrm{Xaa} 28$

$3 \mathrm{C}=1, \mathrm{~L}=2, \mathrm{~F}=9548, \mathrm{~S}=1, \mathrm{Xaa}=28$ 


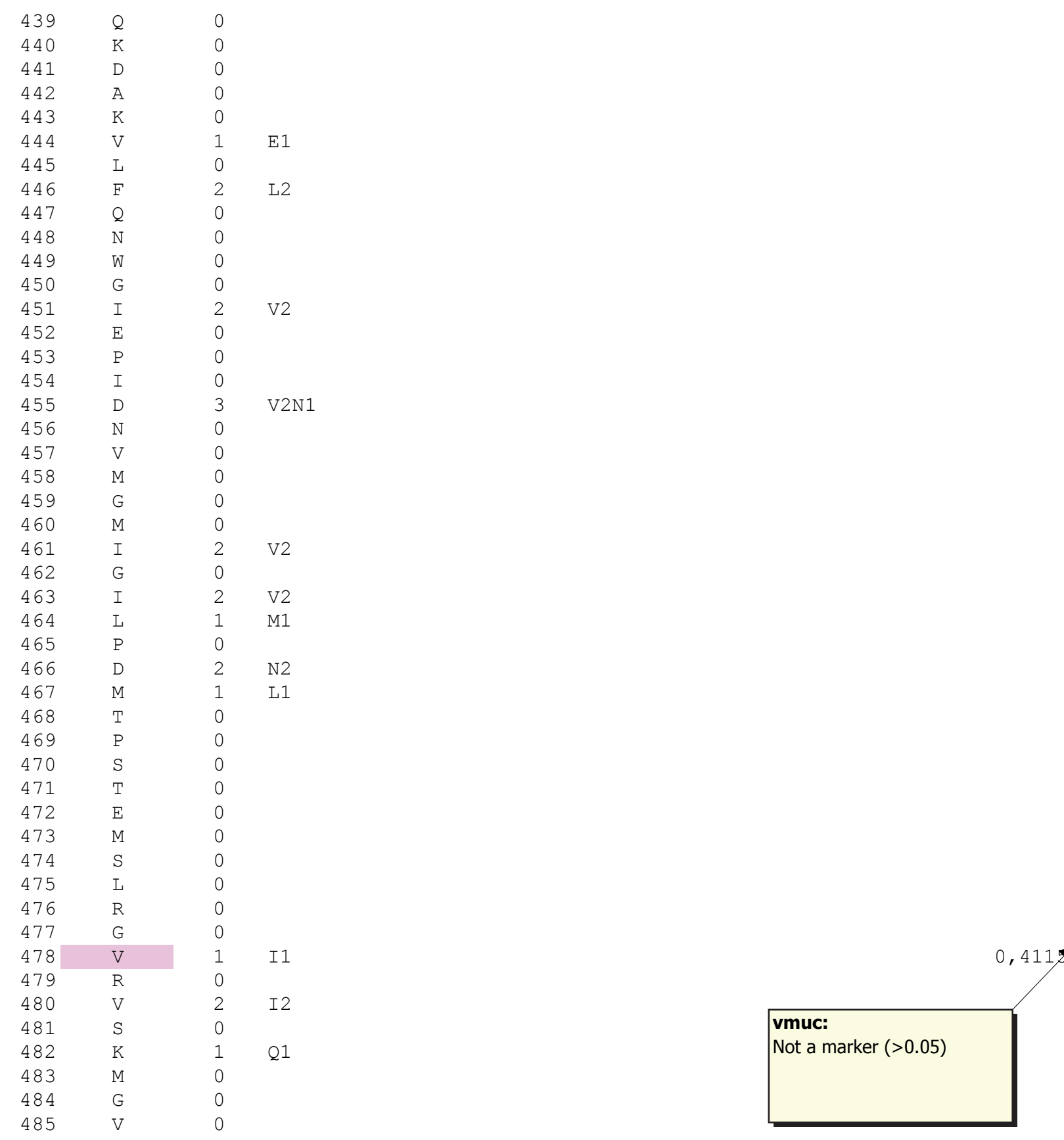

$4 \mathrm{R}=1, \mathrm{Q}=9543, \mathrm{H}=4, \mathrm{P}=2, \mathrm{Xaa}=30$

,

$5 \mathrm{~A}=9540, Q=1, \mathrm{~S}=9, \mathrm{~T}=1, \mathrm{~V}=1, \mathrm{Xaa}=28$

$12 \mathrm{~A}=2, \mathrm{R}=1, \mathrm{E}=2, \mathrm{I}=54, \mathrm{~L}=7, \mathrm{M}=16, \mathrm{~T}=4, \mathrm{~V}=9466, \mathrm{Xaa}=28$

$3 \mathrm{~L}=9553, \mathrm{P}=1, \mathrm{Xaa}=26$
$3 \mathrm{~L}=3, \mathrm{~F}=9549, \mathrm{~S}=2, \mathrm{Xa}=-2$

$8 \mathrm{Q}=9504, \mathrm{H}=44, \mathrm{~L}=2, \mathrm{~K}=3, \mathrm{Xaa}=27$

$19 \mathrm{~N}=9371, \mathrm{D}=12, \mathrm{E}=1, \mathrm{H}=8, \mathrm{~K}=12, \mathrm{~S}=137, \mathrm{~T}=10, \mathrm{Y}=1, \mathrm{Xaa}=28$

$3 \mathrm{E}=1, \mathrm{G}=9552, \mathrm{X} a \mathrm{a}=27$

$72 \mathrm{~N}=3, \mathrm{I}=8306, \mathrm{~L}=16, \mathrm{M}=2, \mathrm{~T}=429, \mathrm{~V}=798, \mathrm{Xaa}=26$

$3 \mathrm{E}=9552, \mathrm{G}=2, \mathrm{~K}=1, \mathrm{Xaa}=25$

$50 \mathrm{~A}=45, \mathrm{R}=1, \mathrm{Q}=10, \mathrm{H}=70, \mathrm{I}=1, \mathrm{~L}=27, \mathrm{P}=8925, \mathrm{~S}=348, \mathrm{~T}=125, \mathrm{Y}=3, \mathrm{Xaa}=25$

$4 \mathrm{I}=9544, \mathrm{~L}=1, \mathrm{M}=1, \mathrm{~F}=1, \mathrm{~S}=1, \mathrm{~T}=3, \mathrm{~V}=4, \mathrm{Xaa}=25$

,

$3 \mathrm{M}=9556, \mathrm{Xaa}=24$

$3 \mathrm{R}=1, \mathrm{G}=9555, \mathrm{Xaa}=24$

(2) $\mathrm{A}=3, \mathrm{I}=9288, \mathrm{~L}=3, \mathrm{xaa}=23$

$\mathrm{T}=2, \mathrm{~V}=258, \mathrm{Xaa}=25$

$15 \mathrm{I}=9417, \mathrm{~L}=21, \mathrm{M}=28, \mathrm{I}$

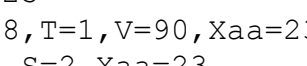

$\mathrm{A} \mathrm{A}=2, \mathrm{H}=9, \mathrm{P}=9500, \mathrm{~S}=45, \mathrm{~T}=1, \mathrm{Xaa}=23$

$4 \mathrm{~A}=1, \mathrm{~N}=5, \mathrm{D}=9546, \mathrm{E}=1, \mathrm{G}=4, \mathrm{Xaa}=23$

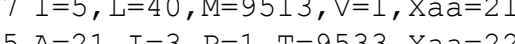

$3 \mathrm{R}=2, \mathrm{~L}=1, \mathrm{P}=9556, \mathrm{Xaa}=21$

$15 \mathrm{R}=2, \mathrm{~N}=154, \mathrm{C}=3, \mathrm{I}=2, \mathrm{~S}=9397, \mathrm{~T}=1, \mathrm{Xaa}=21$

$25 \mathrm{~A}=206, \mathrm{~N}=19, \mathrm{I}=51, \mathrm{M}=2, \mathrm{P}=3, \mathrm{~S}=1, \mathrm{~T}=9277, \mathrm{Xaa}=21$

$64 \mathrm{R}=1, \mathrm{D}=2, \mathrm{E}=4, \mathrm{G}=1, \mathrm{I}=152, \mathrm{I}=209, \mathrm{~K}=8, \mathrm{M}=8710, \mathrm{~T}=136, \mathrm{~V}=334, \mathrm{Xaa}=23$

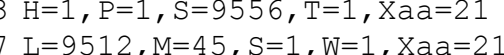

$4 \mathrm{~A}=1, \mathrm{R}=9548, \mathrm{~K}=6, \mathrm{~S}=3, \mathrm{Xaa}=22$

$5 \mathrm{R}=30, \mathrm{G}=9527, \mathrm{Xaa}=23$

$03 \mathrm{~A}=1, \mathrm{I}=5582, \mathrm{~L}=4, \mathrm{M}=30, \mathrm{~V}=3942, \mathrm{Xaa}=21$

$17 \mathrm{~A}=6, \mathrm{C}=1, \mathrm{I}=184, \mathrm{~V}=9371, \mathrm{X} a \mathrm{a}=18$

$2 \mathrm{C}=2, \mathrm{~S}=9560, \mathrm{Xa} a=18$

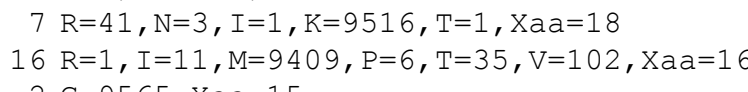

$2 \mathrm{G}=9565, \mathrm{Xaa}=15$
$3 \mathrm{E}=1, \mathrm{G}=1, \mathrm{I}=8, \mathrm{~L}=4, \mathrm{~V}=9551, \mathrm{Xaa}=15$

$2 \mathrm{H}=2, \mathrm{Y}=9561, \mathrm{Xaa}=17$

$6 \mathrm{~A}=9, \mathrm{~F}=2, \mathrm{P}=16, \mathrm{~S}=9532, \mathrm{~T}=2, \mathrm{Y}=3, \mathrm{Xaa}=16$

$10 \mathrm{~A}=67, \mathrm{I}=11, \mathrm{P}=6, \mathrm{~T}=9481, \mathrm{Xa}=15$

$10 \mathrm{~A}=67,1=1, \mathrm{C}=6,1=9481, \mathrm{Xaa}=15$

$5 \mathrm{R}=9528, \mathrm{~K}=37, \mathrm{xaa}=1$

$13 \mathrm{~A}=6, \mathrm{I}=127, \mathrm{~V}=9431, \mathrm{Xaa}=16$

$30 \mathrm{~A}=123, \mathrm{G}=1, \mathrm{I}=153, \mathrm{~L}=3, \mathrm{M}=8, \mathrm{~T}=53, \mathrm{~V}=9223$, Xaa $=16$

$3 \mathrm{~A}=3, \mathrm{E}=1, \mathrm{~L}=5, \mathrm{~F}=1, \mathrm{~V}=9555, \mathrm{Xaa}=15$
$17 \mathrm{R}=6, \mathrm{~N}=192, \mathrm{G}=1, \mathrm{~S}=9365, \mathrm{~T}=2, \mathrm{Xaa}=14$

$3 \mathrm{~N}=1, \mathrm{I}=9557, \mathrm{M}=1, \mathrm{~S}=1, \mathrm{~V}=6, \mathrm{Xaa}=14$

$2 \mathrm{~A}=2, \mathrm{~N}=2, \mathrm{D}=9562, \mathrm{Xa} a=14$

$3 \mathrm{R}=9555, \mathrm{C}=1, \mathrm{Q}=3, \mathrm{H}=5, \mathrm{P}=1, \mathrm{~S}=1, \mathrm{Xa} a=14$

$2 \mathrm{~L}=1, \mathrm{~F}=9564, \mathrm{~S}=1, \mathrm{Xaa}=14$

2 $\mathrm{L}=9561, \mathrm{M}=1, \mathrm{~F}=2, \mathrm{~S}=1, \mathrm{Xaa}=15$

$2 \mathrm{~A}=1, \mathrm{I}=4, \mathrm{~V}=9560, \mathrm{X} a \mathrm{a}=15$

$5 \mathrm{R}=9538, \mathrm{Q}=5, \mathrm{G}=1, \mathrm{H}=5, \mathrm{~K}=14, \mathrm{~W}=2, \mathrm{Xaa}=15$

$2 \mathrm{~N}=1, \mathrm{D}=9562, \mathrm{E}=1, \mathrm{Xaa}=16$

$5 \mathrm{R}=1, \mathrm{Q}=9535, \mathrm{H}=22, \mathrm{~L}=4, \mathrm{~K}=1, \mathrm{P}=1, \mathrm{Xaa}=16$
$51 \mathrm{R}=8803, \mathrm{Q}=594, \mathrm{G}=2, \mathrm{H}=1, \mathrm{~L}=51, \mathrm{~K}=55, \mathrm{M}=51, \mathrm{P}=2, \mathrm{~W}=3, \mathrm{Xaa}=18$

$3 \mathrm{R}=5, \mathrm{~B}=2, \mathrm{G}=9557, \mathrm{Xa} a=16$

$3 \mathrm{R}=5, \mathrm{E}=2, \mathrm{G}=9557, \mathrm{Xaa}=16$
$5 \mathrm{~N}=9530, \mathrm{D}=28, \mathrm{~S}=2, \mathrm{Y}=4, \mathrm{Xaa}=16$

$30 \mathrm{I}=475, \mathrm{~L}=1, \mathrm{M}=3, \mathrm{~V}=9089, \mathrm{Xaa}=12$

$2 \mathrm{I}=1, \mathrm{~L}=9567, \mathrm{~F}=1$, Xaa $=1$

$2 \mathrm{I}=1, \mathrm{~L}=9563, \mathrm{~F}=4, \mathrm{~V}=1, \mathrm{xaa}=11$
$2 \mathrm{~F}=1, \mathrm{P}=1, \mathrm{~S}=9564, \mathrm{~T}=3$, Xaa $=11$

$2 \mathrm{~F}=1, \mathrm{P}=1, \mathrm{~S}=9564, \mathrm{~T}=3, \mathrm{Xaa}=1$
$\mathrm{H}=1, \mathrm{~K}=1, \mathrm{P}=9567, \mathrm{~T}=1, \mathrm{Xaa}=10$

$1 \mathrm{Q}=1, \mathrm{E}=9569, \mathrm{Xa} a=10$

$2 \mathrm{D}=1, \mathrm{Q}=1, \mathrm{E}=9562, \mathrm{G}=2, \mathrm{~K}=2, \mathrm{Xaa}=12$

, $\mathrm{xa} a=11$

$2 \mathrm{R}=1, \mathrm{~N}=1, \mathrm{C}=1, \mathrm{~S}=9563, \mathrm{~T}=1, \mathrm{Xaa}=13$

9 $\mathrm{A}=51, \mathrm{~N}=16, \mathrm{~K}=7, \mathrm{~S}=1, \mathrm{~T}=9492, \mathrm{Xaa}=13$

$5 \mathrm{R}=2, \mathrm{Q}=9539, \mathrm{E}=1, \mathrm{H}=8, \mathrm{I}=18, \mathrm{Xaa}=12$

$2 \mathrm{R}=1, \mathrm{E}=2, \mathrm{G}=9565$, $\mathrm{Xaa}=12$

$24 \mathrm{~A}=30, \mathrm{I}=152, \mathrm{~L}=1, \mathrm{~K}=1, \mathrm{M}=78, \mathrm{~S}=1, \mathrm{~T}=9304, \mathrm{~V}=1$, Xaa $=12$

$2 \mathrm{~A}=1, \mathrm{D}=1, \mathrm{E}=9565, \mathrm{Xaa}=13$

$3 \mathrm{R}=7 \mathrm{~T}, \mathrm{~N}=5, \mathrm{~K}=9485, \mathrm{~T}=4, \mathrm{Xaa}=$

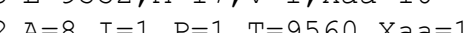

$2 \mathrm{I}=9563, \mathrm{M}=1, \mathrm{~V}=6, \mathrm{Xaa}=10$

$2 \mathrm{~A}=2, \mathrm{I}=5, \mathrm{~T}=9563, \mathrm{Xaa}=10$

$1 \mathrm{Y}=9570, \mathrm{Xaa}=10$
$3 \mathrm{~A}=3, \mathrm{~L}=6, \mathrm{P}=3, \mathrm{~S}=9556, \mathrm{~T}=1, \mathrm{Xaa}=11$

$13 \mathrm{~A}=1, \mathrm{~L}=1, \mathrm{~F}=5, \mathrm{P}=133, \mathrm{~S}=9427, \mathrm{~T}=2, \mathrm{Xaa}=11$

$4 \mathrm{I}=27, \mathrm{~K}=1, \mathrm{M}=9539, \mathrm{~V}=1, \mathrm{Xaa}=12$
$\mathrm{R}=3, \mathrm{I}=2, \mathrm{M}=9561, \mathrm{~V}=1, \mathrm{Xa}=13$

$2 \mathrm{R}=3, \mathrm{I}=2, \mathrm{M}=9561, \mathrm{~V}=1, \mathrm{Xaa}=13$
$2 \mathrm{R}=1, \mathrm{G}=1, \mathrm{~W}=9565, \mathrm{Xaa}=13$

$\mathrm{I}=9529, \mathrm{~L}=5, \mathrm{M}=4, \mathrm{~T}=7, \mathrm{~V}=22, \mathrm{Xaa}=13$

$\mathrm{R}=1, \mathrm{C}=9564, \mathrm{~V}=1, \mathrm{X}=1, \mathrm{Xa}=1$

$5 \mathrm{R}=1, \mathrm{H}=1, \mathrm{~L}=1, \mathrm{P}=9533, \mathrm{~S}=30, \mathrm{xaa}=14$

$9 \mathrm{D}=87, \mathrm{E}=9477, \mathrm{~K}=2, \mathrm{Xaa}=14$
$\mathrm{~L}=1, \mathrm{P}=1, \mathrm{~S}=9565, \mathrm{Xaa}=13$

$2 \mathrm{~L}=1, \mathrm{P}=1, \mathrm{~S}=9565, \mathrm{Xaa}=13$
$\mathrm{I}=18, \mathrm{I}=1, \mathrm{M}=6, \mathrm{~V}=9542, \mathrm{Xaa}=13$

$3 \mathrm{I}=10, \mathrm{~L}=9556, \mathrm{P}=1$, Xaa $=13$

$15 \mathrm{I}=184, \mathrm{~V}=9383$, $\mathrm{Xaa}=13$

$2 \mathrm{~N}=9566, \mathrm{Y}=1, \mathrm{Xaa}=13$
$2 \mathrm{~A}=3, \mathrm{P}=2, \mathrm{~T}=9562, \mathrm{Xaa}=13$

$2 \mathrm{~A}=3, \mathrm{P}=2, \mathrm{~T}=9562, \mathrm{Xaa}=13$
$2 \mathrm{~F}=5, \mathrm{Y}=9562, \mathrm{Xa}=13$

$2 \mathrm{~F}=5, \mathrm{Y}=9562, \mathrm{Xaa}=13$
$2 \mathrm{R}=3, \mathrm{Q}=9563, \mathrm{P}=1, \mathrm{Xaa}=13$

$\mathrm{R}=3, \mathrm{Q}=9563, \mathrm{P}=1, \mathrm{X}$
$1 \mathrm{~W}=9567, \mathrm{Xaa}=13$

$11 \mathrm{~N}=1, \mathrm{I}=9444, \mathrm{~V}=122, \mathrm{Xaa}=13$

$3 \mathrm{I}=9556, \mathrm{M}=1, \mathrm{~S}=3, \mathrm{~T}=2, \mathrm{~V}=4, \mathrm{xaa}=14$

$11 \mathrm{R}=9448, \mathrm{I}=1, \mathrm{~K}=118, \mathrm{Xaa}=13$

The $\mathrm{S}=70, \mathrm{~T}=2, \mathrm{Xa} a=11$

$2 \mathrm{G}=2$, W $=9566, \mathrm{Xaa}=$

$\mathrm{T}=8603, \mathrm{~V}=56, \mathrm{Xaz}=11$

$13 \mathrm{~A}=2, \mathrm{I}=110, \mathrm{~L}=16, \mathrm{M}=6, \mathrm{~T}=1, \mathrm{~V}=9433, \mathrm{Xaa}=12$

$1 \mathrm{~K}=9567, \mathrm{Xaa}=13$

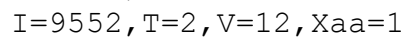

$3 \mathrm{R}=5, \mathrm{Q}=9555, \mathrm{H}=1, \mathrm{~L}=2, \mathrm{~K}=1, \mathrm{Xaa}=1$

$2 \mathrm{~L}=3, \mathrm{~S}=9561, \mathrm{Xaa}=16$

$5 \mathrm{R}=1, \mathrm{Q}=9541, \mathrm{H}=16, \mathrm{~K}=5, \mathrm{P}=1$, $\mathrm{Xaa}=1$

$\mathrm{A}=1, \mathrm{~N}=21, \mathrm{D}=9465, \mathrm{E}=65, \mathrm{G}=7, \mathrm{Y}=1, \mathrm{~V}=4, \mathrm{Xaa}=1$

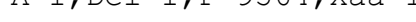

作

$2 \mathrm{Del}=1, \mathrm{I}=1, \mathrm{~L}=9562, \mathrm{P}=1, \mathrm{~S}=1, \mathrm{Xaa}=14$

$2 \mathrm{C}=2, \mathrm{H}=1, \mathrm{Y}=9561, \mathrm{Xaa}=16$

$2 \mathrm{~A}=1, \mathrm{~N}=9561, \mathrm{~K}=2, \mathrm{Xaa}=16$

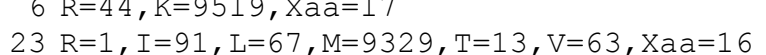

$23 \mathrm{R}=1, \mathrm{I}=91, \mathrm{~L}=\mathrm{X}, \mathrm{M}=93$
$2 \mathrm{E}=9561, \mathrm{~K}=2, \mathrm{Xaa}=17$

$2 \mathrm{~L}=5, \mathrm{~F}=9559, \mathrm{Xaa}=16$

$3 \mathrm{~A}=1, \mathrm{D}=4, \mathrm{E}=9555, \mathrm{G}=2, \mathrm{~K}=1, \mathrm{~S}=1, \mathrm{Xaa}=16$

$4 \mathrm{~A}=1, \mathrm{P}=9547, \mathrm{~S}=15, \mathrm{Xa} a=17$

$2 \mathrm{R}=1, \mathrm{Q}=9563, \mathrm{X} a \mathrm{a}=16$

$2 \mathrm{R}=1, \mathrm{Q}=9563, \mathrm{X} \mathrm{xa}=16$
$3 \mathrm{~A}=2, \mathrm{~F}=1, \mathrm{P}=2, \mathrm{~S}=9558, \mathrm{Y}=2, \mathrm{Xaa}=15$

$6 \mathrm{I}=9518, \mathrm{M}=43, \mathrm{~F}=1, \mathrm{P}=1, \mathrm{~S}=1, \mathrm{xaa}=16$

$14 \mathrm{~A}=1, \mathrm{I}=160, \mathrm{~F}=1, \mathrm{~V}=9402$, $\mathrm{xa} a=16$

$3 \mathrm{~L}=1, \mathrm{P}=9558, \mathrm{~S}=5, \mathrm{Xaa}=16$

$2 \mathrm{~A}=9561, \mathrm{P}=1, \mathrm{~T}=2, \mathrm{X} a \mathrm{a}=16$

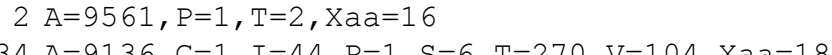




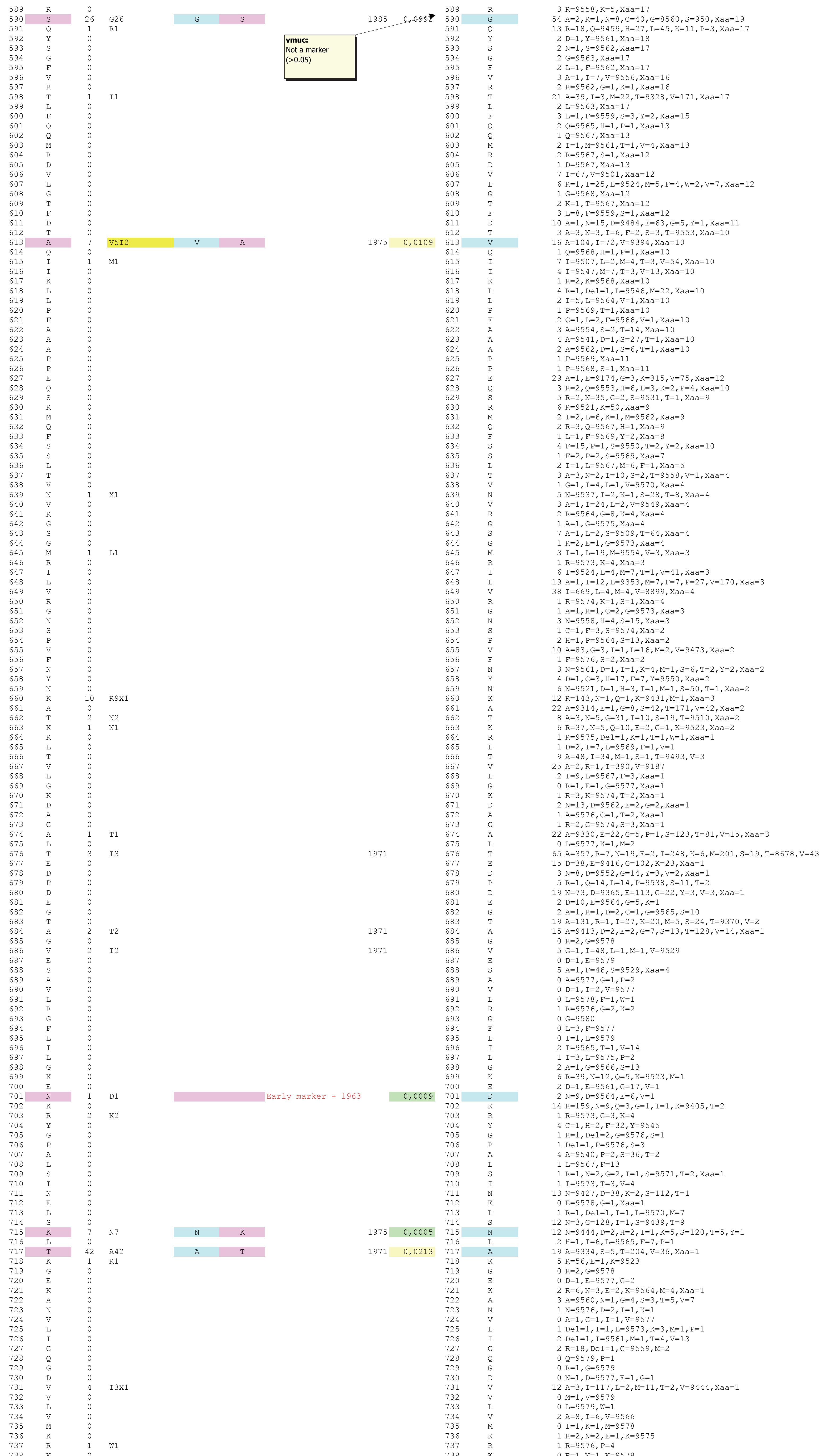


$3 \mathrm{~A}=2, \mathrm{~F}=21, \mathrm{~S}=9556, \mathrm{Xa}=1$

$0 \mathrm{G}=1, \mathrm{~S}=9577, \mathrm{~T}=2$

$1 \mathrm{I}=9, \mathrm{P}=1, \mathrm{~T}=9570$

$1 \mathrm{R}=1, \mathrm{~N}=1, \mathrm{C}=1, \mathrm{G}=1, \mathrm{I}=1, \mathrm{~S}=9575$

$1 \mathrm{R}=1, \mathrm{Q}=9576, \mathrm{H}=1, \mathrm{~L}=1, \mathrm{P}=1$

P $\mathrm{P}=1,1=9579$

$0 \mathrm{~A}=9577, \mathrm{G}=1, \mathrm{P}=2$

I $\mathrm{R}=3, \mathrm{Q}=1, \mathrm{I}=1, \mathrm{~K}=9574, \mathrm{~T}=1$

$1 \mathrm{R}=3, \mathrm{Q}=1, \mathrm{I}=1, \mathrm{~K}=9574$,

$2 \mathrm{H}=1, \mathrm{I}=9561, \mathrm{~L}=3, \mathrm{~F}=3, \mathrm{~T}=2, \mathrm{~V}=10$

$5 \mathrm{R}=4, \mathrm{D}=1, \mathrm{I}=4, \mathrm{~L}=7, \mathrm{M}=9537, \mathrm{~S}=1, \mathrm{~T}=6, \mathrm{~V}=20$

$5 \mathrm{~N}=2, \mathrm{I}=9528, \mathrm{M}=1, \mathrm{~F}=2, \mathrm{~T}=44, \mathrm{~V}=2, \mathrm{Xaa}=1$ 



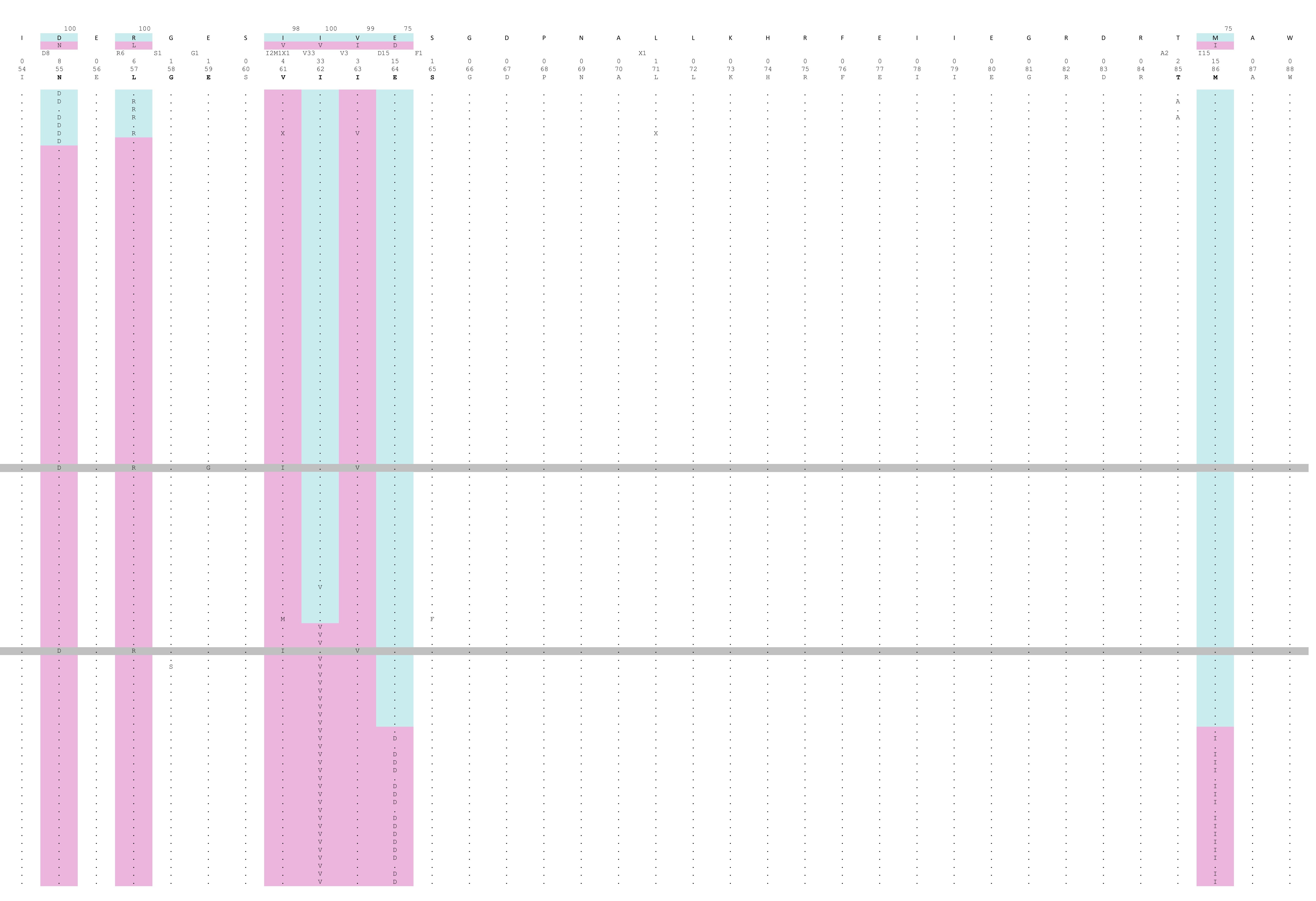


<smiles>CC1(C)C(C)(C)C(C)(C)C1(C)C</smiles> 



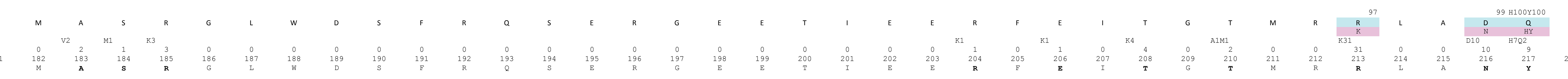
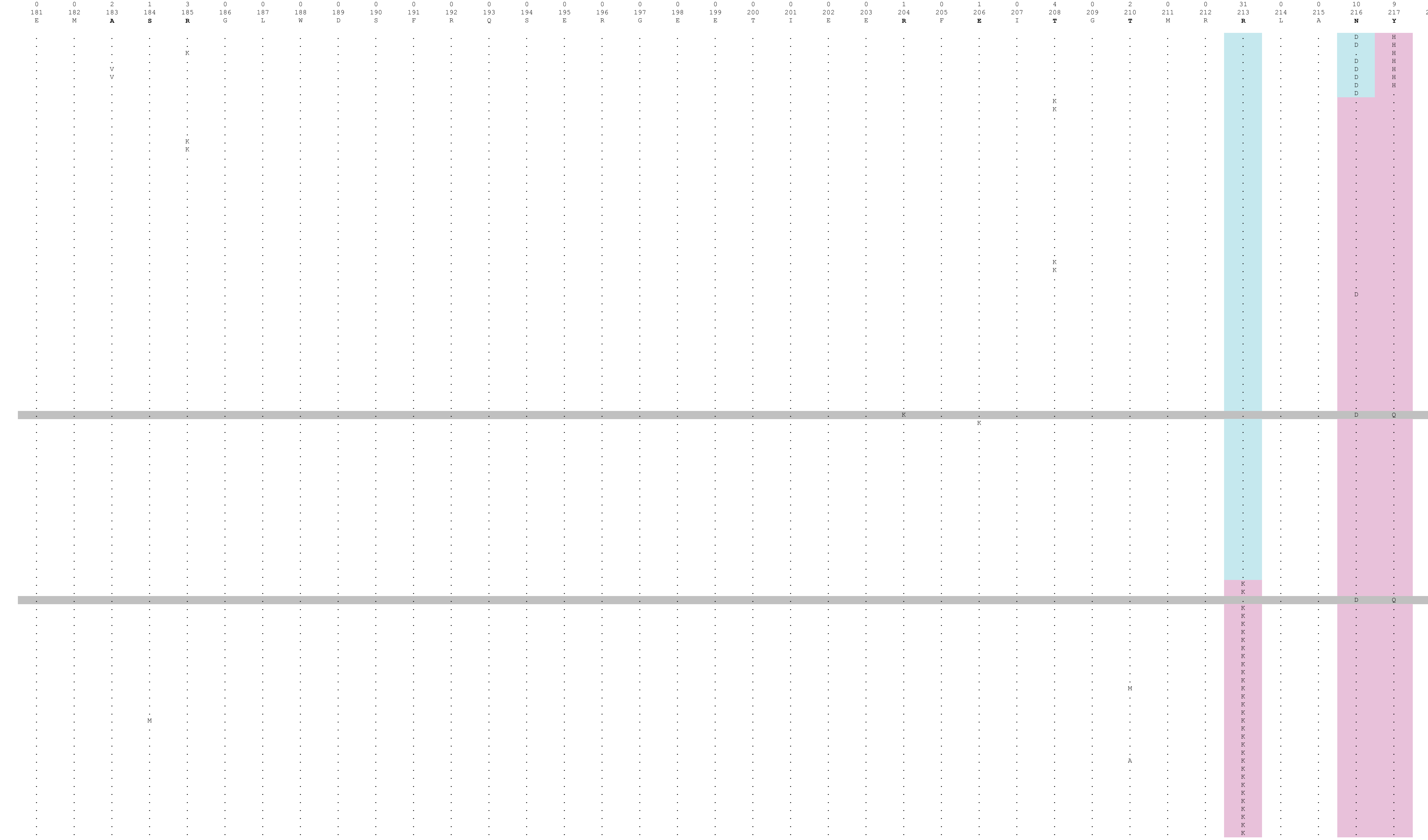


$$
\text { | }
$$




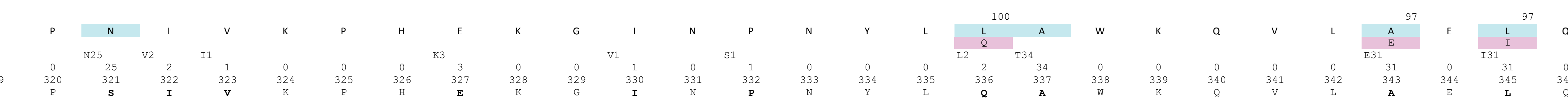










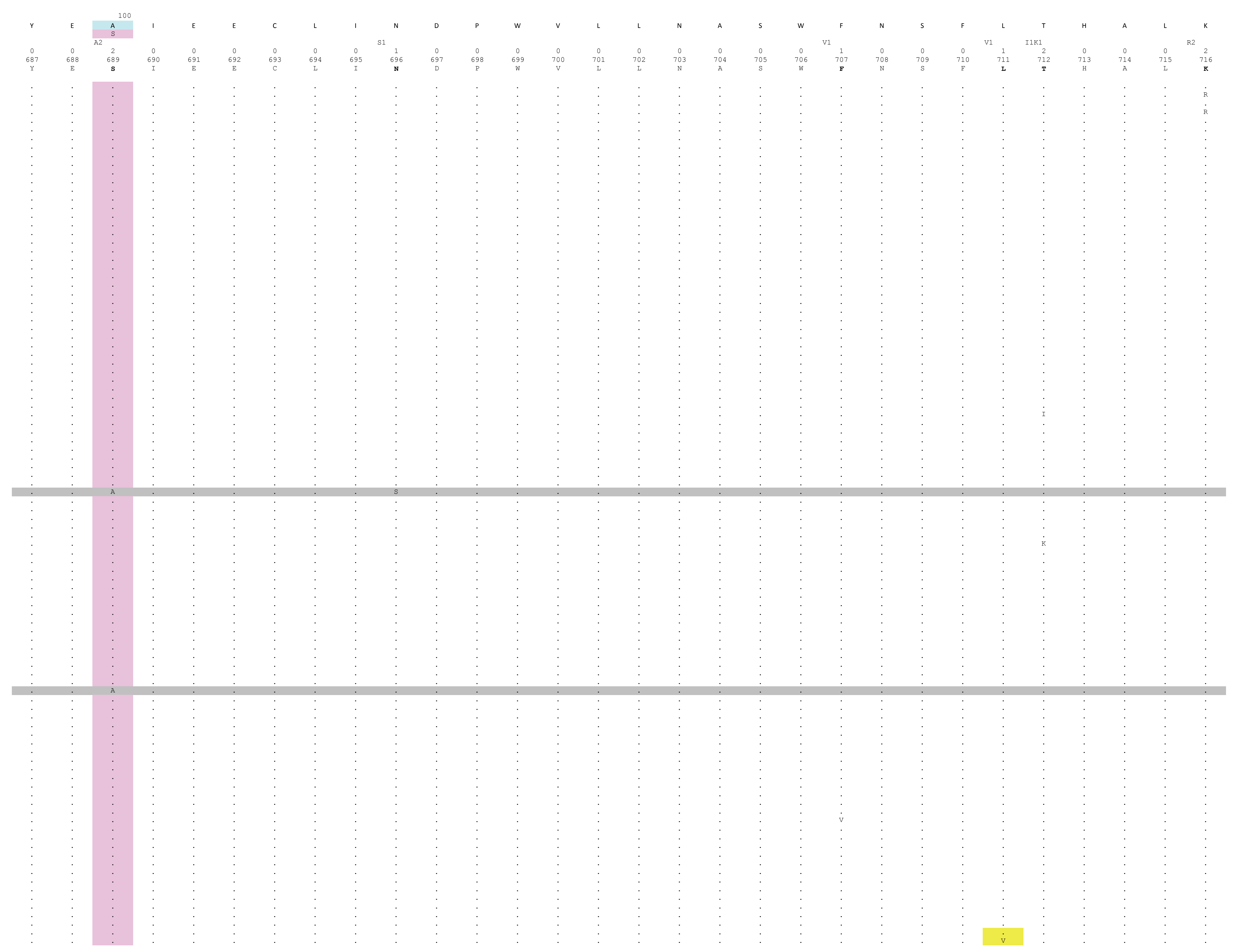




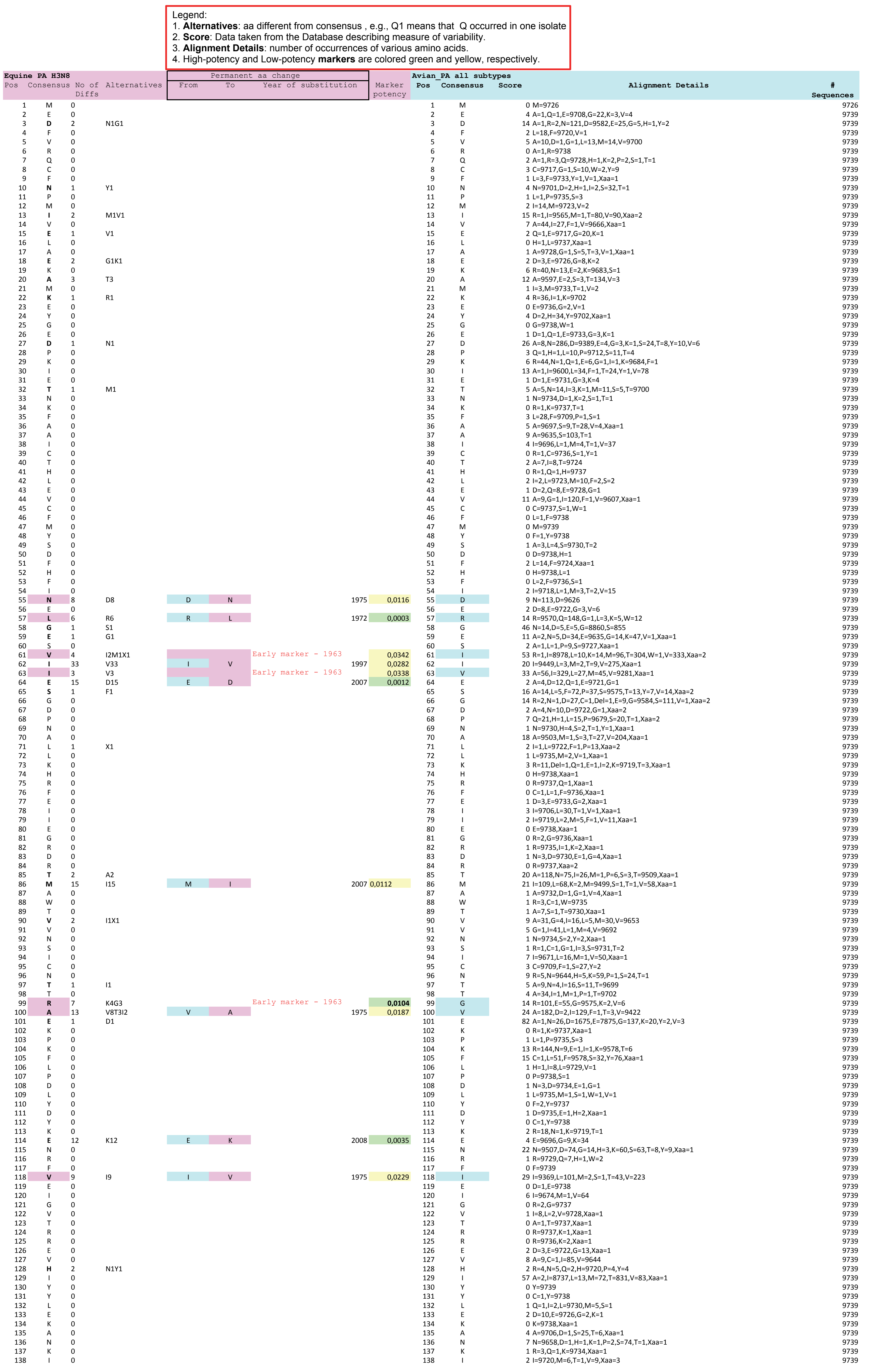




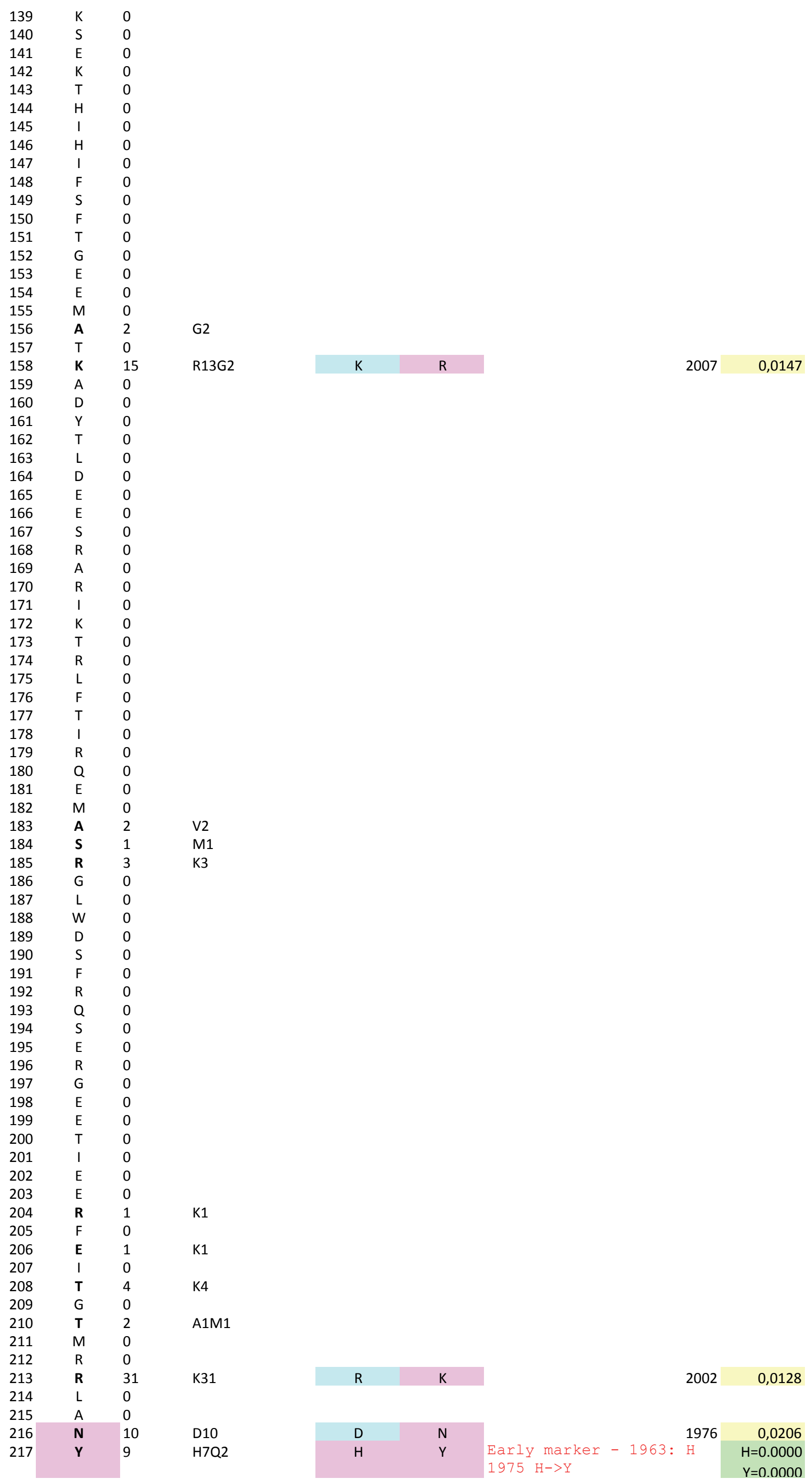

$1 \mathrm{R}=2, \mathrm{~N}=2, \mathrm{~K}=9734, \mathrm{Xaa}=1$

S $=9618, T=90, X a a=1$

$20 \mathrm{R}=168, \mathrm{~N}=54, \mathrm{D}=4, \mathrm{Q}=6 \mathrm{6}, \mathrm{E}=\mathrm{Xa} \mathrm{K}=9494, \mathrm{M}=4, \mathrm{~T}=1, \mathrm{Xaa}=2$

$1 A=3, M=1, P=1, T=9733$, xaa $=1$

$0 \mathrm{H}=9737, \mathrm{P}=1, \mathrm{Xaa}=1$

$1 \mathrm{l}=9730, \mathrm{M}=2, \mathrm{~F}=1, \mathrm{~V}=3, \mathrm{Xaa}=3$

$1 \mathrm{~L}=2, \mathrm{~F}=9733, \mathrm{Y}=2, \mathrm{Xaa}=2$

1 $A=9, N=70,1=3, S=3, T=9615, X a a=3$

$\mathrm{E}=1, \mathrm{G}=9736, \mathrm{Xaa}=2$

$1 \mathrm{~A}=1, \mathrm{Del}=1, \mathrm{E}=9729, \mathrm{G}=2, \mathrm{~K}=5, \mathrm{Xaa}=1$

2 Del=1, $=2,2, L=4, M=9727, \mathrm{~T}=1, \mathrm{~V}=1, \mathrm{Xaa}=3$

4 A A $=9698$, Del $=1, T=6, V=33$, Xaa 1

$5 \mathrm{R}=143, \mathrm{~N}=1, \mathrm{Q}=20, \mathrm{E}=2, \mathrm{G}=5, \mathrm{~K}=9563, \mathrm{M}=1, \mathrm{~T}=1, \mathrm{Xaa}=3$

$7 \mathrm{~A}=9676, \mathrm{C}=1, \mathrm{E}=19, \mathrm{~S}=24, \mathrm{~T}=14, \mathrm{~V}=3, \mathrm{Xaa}=2$

$10 \mathrm{~N}=34, \mathrm{D}=9639, \mathrm{E}=58, \mathrm{G}=5, \mathrm{~K}=1, \mathrm{Xaa}=2$

$5 \mathrm{~A}=4, \mathrm{~N}=2, \mathrm{l}=30, \mathrm{~S}=3, \mathrm{~T}=9697, \mathrm{Xaa}=3$

$1 \mathrm{~N}=1, \mathrm{D}=9730, \mathrm{G}=2, \mathrm{H}=1, \mathrm{~S}=1, \mathrm{~V}=1, \mathrm{Xaa}=3$
$3 \mathrm{~A}=1, \mathrm{D}=16, \mathrm{E}=9716, \mathrm{G}=1, \mathrm{~S}=1, \mathrm{Xaa}=4$

$1 \mathrm{E}=9731, \mathrm{~K}=5, \mathrm{Xaa}=3$

$1 \mathrm{~N}=1, \mathrm{l}=1, \mathrm{~S}=9734, \mathrm{Xaa}=3$

$1 \mathrm{~A}=9731, \mathrm{G}=1, \mathrm{P}=4, \mathrm{Xaa}=3$

$1 \mathrm{R}=9733, \mathrm{G}=3, \mathrm{Xaa}=3$

$5 \mathrm{l}=9698, \mathrm{~L}=1, \mathrm{~K}=1, \mathrm{M}=1, \mathrm{~F}=2, \mathrm{~T}=2, \mathrm{~V}=31$, Xaa=3

$1 \mathrm{~N}=3, \mathrm{~K}=9733, \mathrm{Xaa}=3, \mathrm{~S}=2, \mathrm{~T}$

$1 \mathrm{R}=1, \mathrm{Q}=1, \mathrm{~L}=9730, \mathrm{M}=3, \mathrm{P}=1, \mathrm{Xaa}=3$

$3 \mathrm{C}=1, \mathrm{~L}=1, \mathrm{~F}=9708 \mathrm{~S}, \mathrm{~S}=1, \mathrm{Y}=25, \mathrm{Xaa}=3$

$1 \mathrm{~A}=2, \mathrm{~N}=2, \mathrm{P}=2, \mathrm{~T}=9730, \mathrm{Xaa}=3$
$\mathrm{l}=9722, \mathrm{~K}=1 \mathrm{~T}=2, \mathrm{~V}=11, \mathrm{Xaa}=3$

$2 \mathrm{l}=9722, \mathrm{~K}=1, \mathrm{~T}=2, \mathrm{~V}=11, \mathrm{X}$
$1 \mathrm{R}=9731, \mathrm{~S}=5, \mathrm{X}$ aa $=3$

$2 \mathrm{R}=1, \mathrm{Q}=9727, \mathrm{E}=1, \mathrm{H}=4, \mathrm{~K}=1, \mathrm{P}=2 \mathrm{X}$ Xaa $=3$

$2 \mathrm{~A}=9727, \mathrm{D}=1, \mathrm{G}=1, \mathrm{P}=1, \mathrm{~S}=2, \mathrm{~T}=3 \mathrm{~V}, \mathrm{~V}=1, \mathrm{Xaa}=3$

$16 \mathrm{R}=2, \mathrm{~N}=90, \mathrm{G}, \mathrm{G}=34, \mathrm{l}, \mathrm{l}=3, \mathrm{~S}, \mathrm{~S}=95 \mathrm{~S}=6, \mathrm{~T}=9, \mathrm{X}, \mathrm{Xaa}=3$

$4 \mathrm{R}=9699, \mathrm{G}=2, \mathrm{~K}=31, \mathrm{M}=1, \mathrm{~S}=1, \mathrm{Xaa}=5$
$8 \mathrm{D}=1, \mathrm{G}=9649, \mathrm{~S}=85, \mathrm{X}=4$

$8 \mathrm{D}=1, \mathrm{G}=9649, \mathrm{~S}=85, \mathrm{Xaa}=4$
$6 \mathrm{I}=54, \mathrm{~L}=9673, \mathrm{M}=8, \mathrm{~F}=1, \mathrm{Xa}=3$

$0 \mathrm{~W}=9736, \mathrm{Xaa}=3$

$2 \mathrm{~N}=1, \mathrm{D}=9 \mathrm{9} 20 \mathrm{~V}=\mathrm{E}=8, \mathrm{G}=5, \mathrm{~V}=1, \mathrm{Xaa}=4$
$7 \mathrm{~F}=2, \mathrm{P}=65, \mathrm{~S}=9667, \mathrm{~T}=1, \mathrm{Y}=1, \mathrm{Xaa}=3$

$1 \mathrm{~L}=1, \mathrm{~F}=9735, \mathrm{Xaa}=3$

作 $\mathrm{R}=2, \mathrm{Q}=9726, \mathrm{H}=3, \mathrm{~L}=3, \mathrm{P}=1, \mathrm{~S}=1, \mathrm{X}$ Xaa $=3$

$1 \mathrm{~A}=1, \mathrm{~F}=2, \mathrm{~S}=9732, \mathrm{Y}=1, \mathrm{Xaa}=3$

$1 \mathrm{R}=1, \mathrm{Q}=1, \mathrm{E}=9734, \mathrm{Xaa}=3$

$2 \mathrm{R}=9727, \mathrm{G}=1, \mathrm{l}, \mathrm{I}, \mathrm{K}=3, \mathrm{~S}=3, \mathrm{Xaa}=3$

$2 \mathrm{D}=6, \mathrm{C}=1, \mathrm{G}=9727, \mathrm{~S}=2, \mathrm{X}$

$15 \mathrm{~A}=1, \mathrm{D}=172, \mathrm{E}=9545, \mathrm{G}=11, \mathrm{~K}=7, \mathrm{Xaa}=3$

$1 \mathrm{~A}=1, \mathrm{D}=1, \mathrm{l}=2, \mathrm{~T}=9732, \mathrm{Xaa}=3$
$20 \mathrm{~N}=2, \mathrm{l}=\mathrm{N} 486, \mathrm{~L}=2, \mathrm{M}=6, \mathrm{~S}=2, \mathrm{~T}=19, \mathrm{~V}=217, \mathrm{Xaa}=5$

$1 \mathrm{Q}=2, \mathrm{E}=9734, \mathrm{Xaa}=3$

$1 \mathrm{E}=97 \mathrm{R}=9, \mathrm{G}=1, \mathrm{Xaa}=3$
$43 \mathrm{R}=8897, \mathrm{G}=4, \mathrm{~K}=831, \mathrm{~S}=2, \mathrm{Xaa}=5$

$1 \mathrm{~L}=2, \mathrm{~F}=9734$, Xaa $=3$

$5 \mathrm{~A}=1, \mathrm{D}=23, \mathrm{E}=9698, \mathrm{G}=6, \mathrm{~K}=8, \mathrm{Xaa}=3$

$3 \mathrm{l}=9716, \mathrm{~L}=15, \mathrm{~V}=5, \mathrm{Xaa}=3$

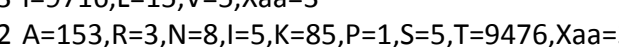

$7 \mathrm{~A}=53, \mathrm{~N}=4, \mathrm{D}=1, \mathrm{~L}=2, \mathrm{~S}=2 \mathrm{~S}=2 \mathrm{~T}=\mathrm{T}=1, \mathrm{X}, \mathrm{Xaa}=4$

$8 \mathrm{~B}=5, \mathrm{~L}=9, \mathrm{M}=96 \mathrm{G} 7 \mathrm{~T}=1 \mathrm{~V}=12 \mathrm{X}, \mathrm{X}=4$

$5 \mathrm{R}=9695, \mathrm{C}=11, \mathrm{H}=23, \mathrm{~L}=5, \mathrm{P}=1, \mathrm{Xaa}=4$

$12 \mathrm{R}=9597, \mathrm{G}=1, \mathrm{l}=1, \mathrm{~K}=125, \mathrm{M}=7, \mathrm{~S}=4, \mathrm{Xaa}=4$

3) $1=17, L=9710, F=5, P=1, S=1, X a a=5$

$1 \mathrm{~A}=9729, \mathrm{~S}=1, \mathrm{~T}=3, \mathrm{~V}=1, \mathrm{Xaa}=5$
$\mathrm{~A}=1, \mathrm{~N}=201, \mathrm{D}=9464, \mathrm{E}=5 \mathrm{~S}, \mathrm{G}=8, \mathrm{~S}=3, \mathrm{Y}=2, \mathrm{Xaa}=5$

$1 \mathrm{R}=2, \mathrm{Q}=9730, \mathrm{~K}=1, \mathrm{P}=1, \mathrm{Xaa}=5$

$1 \mathrm{R}=1, \mathrm{G}=1, \mathrm{l}=2, \mathrm{~S}=9729, \mathrm{Xaa}=6$

$2 \mathrm{G}=1, \mathrm{l}=10, \mathrm{~L}=9720, \mathrm{~F}=1, \mathrm{P}=1, \mathrm{Xaa}=6$

$1 \mathrm{P}=9728, \mathrm{~S}=2, \mathrm{~T}=1, \mathrm{X} \mathrm{Xa}=8$
$3 \mathrm{R}=2, \mathrm{Q}=2, \mathrm{~L}=5, \mathrm{P}=9712, \mathrm{~S}=7, \mathrm{~T}=2, \mathrm{Xaa}=9$

$3 \mathrm{R}=2, \mathrm{Q}=2, \mathrm{~L}=5, \mathrm{P}=9 \mathrm{~V}=2 \mathrm{~K}, \mathrm{~S}=\mathrm{S}=\mathrm{T}=2, \mathrm{Xad}=9$
$\mathrm{~N}=9702, \mathrm{D}=2, \mathrm{H}=2, \mathrm{~K}=2, \mathrm{~S}=20, \mathrm{~T}=2, \mathrm{Y}=1, \mathrm{Xaa}=8$

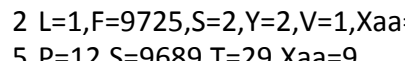

$5 \mathrm{P}=12, \mathrm{~S}=9689, \mathrm{~T}=29, \mathrm{Xaa}=9$

$5 \mathrm{R}=1, \mathrm{~N}=15, \mathrm{C}=13, \mathrm{G}=3, \mathrm{~S}=9699, \mathrm{Xaa}=8$

(8) $R=1, Q=1,1=11, L=95,00, F=217, P=1, x a a=8$

$4 \mathrm{~N}=9703, \mathrm{D}=10, \mathrm{H}=3, \mathrm{~K}=7, \mathrm{~S}=4, \mathrm{~T}=3, \mathrm{Xaa}=9$

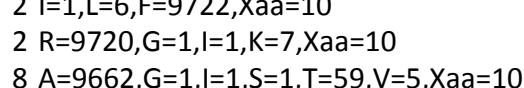

$8 \mathrm{~A}=9662, \mathrm{G}=1, \mathrm{l}=1, \mathrm{~S}=1, \mathrm{~T}=5 \mathrm{~T}, \mathrm{~V}$

$1 \mathrm{~N}=1, \mathrm{C}=1, \mathrm{Y}=9728, \mathrm{X}, \mathrm{W}$
$1 \mathrm{~V}=9729, \mathrm{Xaa}=10$

$1 \mathrm{~N}=1, \mathrm{D}=9727, \mathrm{E}=1, \mathrm{Xaa}=10$

2 R=4,G=9724,Xaa=11

$2 \mathrm{C}=1, \mathrm{~F}=9725, \mathrm{~S}=1, \mathrm{Y}=1, \mathrm{Xaa}=11$

19 $\mathrm{Q}=2, \mathrm{E}=9502, \mathrm{G}=12, \mathrm{~K}=201, \mathrm{~V}=9, \mathrm{Xaa}=13$

$2 \mathrm{~L}=3, \mathrm{P}=9722, \mathrm{~S}=1, \mathrm{~T}=1, \mathrm{X}$ aad $=12$
$3 \mathrm{~N}=9715 \mathrm{D}=1, \mathrm{X}$

$2 G=9721, S=6, X 20=12$,

$14 \mathrm{R}=2, \mathrm{C}=9579, \mathrm{~F}=11, \mathrm{~S}=18, \mathrm{~W}=1, \mathrm{Y}=115, \mathrm{Xaa}=13$

$2 \mathrm{l}=9722, \mathrm{~L}=3, \mathrm{~F}=1, \mathrm{Xaa}=13$

$2 \mathrm{D}=2, \mathrm{E}=9722, \mathrm{G}=1, \mathrm{~K}=1, \mathrm{Xaa}=13$

2 $\mathrm{G}=9723, \mathrm{~S}=2, \mathrm{~V}=1, \mathrm{Xaa}=13$

$2 \mathrm{~L}=9724, \mathrm{P}=1, \mathrm{Xaa}=14$

$5 \mathrm{~A}=18, \mathrm{~L}=1, \mathrm{~F}=3, \mathrm{P}=2, \mathrm{~S}=9697, \mathrm{Y}=1, \mathrm{Xaa}=17$

$2 \mathrm{Q}=9719, \mathrm{H}=1, \mathrm{~L}=1, \mathrm{~K}=1, \mathrm{P}=2, \mathrm{Xaa}=15$

$2 \mathrm{l}=1, \mathrm{M}=9721, \mathrm{~W}=1, \mathrm{~V}=1, \mathrm{xaa}=15$

$6 L=2, F=1, P=36, S=9684, T=1, \times a a=15$
$5 R=22, N=1, Q=1, E=1, K=9697, M=1, X=16$

$6 \mathrm{~A}=11, \mathrm{~N}=34, \mathrm{D}=12, \mathrm{E}=9572, \mathrm{G}=17, \mathrm{~K}=77, \mathrm{Xaa}=1$

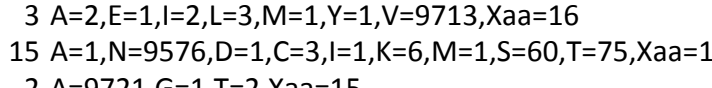

$2 \mathrm{~A}=9721, \mathrm{G}=1, \mathrm{~T}=2, \mathrm{Xaa}=15$

A $N=1,1=9701, V=22, X, K=63, T=2, W=1, X a a=16$

$4 \mathrm{D}=18, \mathrm{E}=9702, \mathrm{~K}=2, \mathrm{~V}=2, \mathrm{X} \mathrm{a} a=15$

2 $A=1, Q=86, L=1, P=9611, S=20, T=5, X a a=15$

$2 \mathrm{C}=1, \mathrm{~F}=9721, \mathrm{~S}=1, \mathrm{~V}=1, \mathrm{Xaa}=15$

$41 \mathrm{Q}=58, \mathrm{l}=1, \mathrm{~L}=9148, \mathrm{M}=443, \mathrm{~F}=14, \mathrm{~S}=13, \mathrm{~T}=7, \mathrm{~W}=1, \mathrm{~V}=38, \mathrm{Xaa}=1$

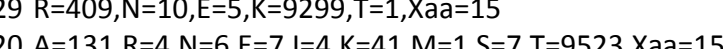

$2 \mathrm{G}=1, \mathrm{~K}=1, \mathrm{~T}=9721, \mathrm{Xaa}=16$

$9 \mathrm{R}=9654, \mathrm{C}=16, \mathrm{Q}=9, \mathrm{H}=32, \mathrm{~L}=2, \mathrm{P}=2, \mathrm{~S}=6, \mathrm{Xaa}=18$

$13 \mathrm{C}=1, \mathrm{H}=21, \mathrm{~L}=9, \mathrm{P}=9609, \mathrm{~S}=77, \mathrm{~T}=4, \mathrm{Xaa}=18$

$91 \mathrm{R}=6935, \mathrm{E}=2, \mathrm{G}=3, \mathrm{I}=14, \mathrm{~K}=27 \mathrm{~K}=278 \mathrm{P}, \mathrm{P}=1, \mathrm{~T}=1, \mathrm{Xaa}=25$

4 Del=1,l=7,L=9703,M=3,F=3,T=1,Xaa=21

$4 \mathrm{R}=1, \mathrm{~L}=1, \mathrm{~F}=1, \mathrm{P}=9706, \mathrm{~S}=8, \mathrm{~T}=1, \mathrm{Xaa}=21$

政 $2041, \mathrm{G}=160, \mathrm{~K}=15, \mathrm{~F}=1, \mathrm{~S}=25, \mathrm{Y}=1, \mathrm{~V}=11, \mathrm{Xaa}=22$ $3 \mathrm{E}=1, \mathrm{G}=9715, \mathrm{~F}=1, \mathrm{~W}=1, \mathrm{Xaa}=21$

$1 \mathrm{~A}=8, \mathrm{R}=2, \mathrm{H}=7, \mathrm{~L}=44, \mathrm{P}=9629, \mathrm{~S}=25, \mathrm{~T}=1, \mathrm{Xaa}=23$

$3 \mathrm{C}=9715, \mathrm{Q}=1, \mathrm{Xaa}=23$

$21 \mathrm{~A}=16, \mathrm{C}, \mathrm{C}=5, \mathrm{H}=6, \mathrm{~L}=1, \mathrm{~K}=1, \mathrm{~F}=120, \mathrm{P}=13, \mathrm{~S}=9514, \mathrm{~T}=8, \mathrm{Y}=29, \mathrm{Xaa}=26$

$3 \mathrm{Q}=9712, \mathrm{H}=1, \mathrm{~L}=1, \mathrm{~K}=1, \mathrm{Xaa}=24$

3 $\mathrm{A}=\mathrm{g}, \mathrm{B}, \mathrm{Q}=3, \mathrm{G}=1, \mathrm{H}=1, \mathrm{~K}=1, \mathrm{P}=1, \mathrm{Xaa}=2$

$3 \mathrm{G}=1, \mathrm{~K}=9714, \mathrm{Xaa}=24$

$3 \mathrm{l}=1, \mathrm{~L}=1, \mathrm{~F}=9712, \mathrm{~S}=1, \mathrm{Xaa}=2$

$3 \mathrm{~L}=9711, \mathrm{M}=2, \mathrm{~F}=1, \mathrm{~W}=1, \mathrm{Xaa}=24$

$5 \mathrm{R}=1, \mathrm{~L}=9698, \mathrm{M}=14, \mathrm{~F}=1, \mathrm{P}=1, \mathrm{Xaa}=24$

$3 \mathrm{~N}=1, \mathrm{D}=9713, \mathrm{~K}=1, \mathrm{Xaa}=24$

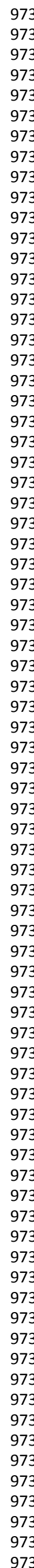

9739

9759

9739

9739

979

9739
9739
9739

9739

9739

9739

9739
9739
9739

979

9779
9739
9799

9739
9739
9

9739
9739
9739

9739

9739
9739

9739
9739
9739

9739
9739
9739

9739
9739
9739

9739

9739

9739
9739

9739
9739
97339

9739

9739
9739
9739

9739

9739

9739
9739
9739 


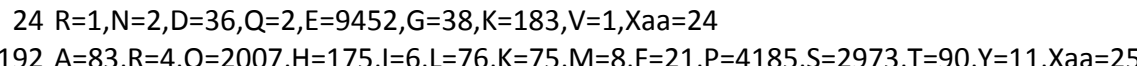

$15 \mathrm{R}=9550, \mathrm{~N}=1, \mathrm{G}=2, \mathrm{l}=1, \mathrm{~K}=159, \mathrm{~S}=3, \mathrm{X}, \mathrm{K}=23$

$3 \mathrm{~A}=6, \mathrm{~S}=9709, \mathrm{~T}=2, \mathrm{Xaa}=22$

$7 \mathrm{l}=36, \mathrm{~L}=9668, \mathrm{~F}=1, \mathrm{P}=10, \mathrm{~S}=1, \mathrm{~V}=1, \mathrm{Xaa}=22$

$44 \mathrm{~A}=8992, \mathrm{E}=1, \mathrm{G}=1, \mathrm{P}=4, \mathrm{~S}=669, \mathrm{~T}=41, \mathrm{~V}=8, \mathrm{Xaa}=23$
$22 \mathrm{~A}=52, \mathrm{~N}=7, \mathrm{C}=137, \mathrm{G}=7, \mathrm{I}=9, \mathrm{~S}=9489, \mathrm{~T}=16, \mathrm{Xaa}=22$

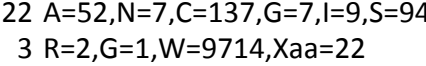

$\begin{array}{rl}3 & \mathrm{R}=2, \mathrm{G}=1, \mathrm{~W}=9714, \mathrm{Xaa}=22 \\ 24 \mathrm{~A}=3, \mathrm{l}=9417, \mathrm{~T}=24, \mathrm{~V}=273, \mathrm{Xa}=22\end{array}$

$2 \mathrm{Q}=9716, \mathrm{P}=1, \mathrm{Xaa}=22$

$43 \mathrm{R}=2, \mathrm{~N}=723, \mathrm{G}=16, \mathrm{~K}=1, \mathrm{~S}=8974, \mathrm{Xaa}=23$

$3 \mathrm{E}=9714, \mathrm{G}=1, \mathrm{~V}=2, \mathrm{Xaa}=22$
$3 \mathrm{~F}=9716, \mathrm{Y}=3, \mathrm{Xaa}=20$

$3 \mathrm{~F}=9716, \mathrm{Y}=3, \mathrm{Xaa}=20$

$2 \mathrm{~N}=9719, \mathrm{Xaa}=20$
$3 \mathrm{R}=8, \mathrm{~K}=9710, \mathrm{Xaa}=21$

$3 \mathrm{~A}=9715, \mathrm{G}, \mathrm{X}, \mathrm{T}=2, \mathrm{Xaa}=21$

$2 \mathrm{C}=9717, \mathrm{~W}=1, \mathrm{X}$ aa $=21$

$3 \mathrm{D}=1, \mathrm{E}=9716, \mathrm{~K}=1, \mathrm{Xaa}=21$
$3 \mathrm{l}=1, \mathrm{~L}=9715, \mathrm{~V}=2, \mathrm{Xaa}=21$

$3 \mathrm{l}=1, \mathrm{~L}=9715, \mathrm{~V}=2, \mathrm{Xaa}=21$
$3 \mathrm{~A}=6, \mathrm{~T}=9712, \mathrm{Xaa}=21$

$12 \mathrm{~N}=39, \mathrm{D}=9614, \mathrm{E}=17, \mathrm{G}=47, \mathrm{~V}=1$

$3 \mathrm{~A}=1, \mathrm{P}=1, \mathrm{~S}=9714, \mathrm{~T}=2, \mathrm{Xaa}=21, \mathrm{Xaa}=2$

$9 \mathrm{~N}=12, \mathrm{G}=15, \mathrm{l}=34, \mathrm{~S}=9655, \mathrm{~T}=1, \mathrm{Xaa}=22$

$2 \mathrm{~W}=9717, \mathrm{Xaa}=22$

$29 \mathrm{~A}=1, \mathrm{l}=9378, \mathrm{~L}=21, \mathrm{M}=187, \mathrm{~T}=4, \mathrm{~V}=126, \mathrm{Xaa}=22$

$4 \mathrm{~L} D=9, \mathrm{E}=9707, \mathrm{~V}=1, \mathrm{X}, \mathrm{Xaa}=22$
$5 \mathrm{~L}=9693, \mathrm{M}=1, \mathrm{~F}=23, \mathrm{Xaa}=22$

$5 \mathrm{~L}=9693, \mathrm{M}=1, \mathrm{~F}=23, \mathrm{Xaa}=22$
$3 \mathrm{D}=9707, \mathrm{E}=10, \mathrm{Xaa}=22$

$3 \mathrm{D}=1, \mathrm{E}=9714, \mathrm{G}=2, \mathrm{Xaa}=22$
$4 \mathrm{I}=9706, \mathrm{~L}=6, \mathrm{M}=2, \mathrm{~V}=2, \mathrm{Xaa}=23$

$4 \mathrm{l}=9706, \mathrm{~L}=6, \mathrm{M}=2, \mathrm{~V}=2, \mathrm{Xaa}=23$
$3 \mathrm{R}=3, \mathrm{E}=1, \mathrm{G}=9713, \mathrm{Xaa}=22$

$11 \mathrm{~A}=8, \mathrm{D}=37, \mathrm{E}=9638, \mathrm{X}, \mathrm{G}=8, \mathrm{~K}=1, \mathrm{~V}=24, \mathrm{Xaa}=23$

$60 A=3, l=1254, L=17, F=1, V=8443, X a a=2$

$2 \mathrm{~A}=9717, \mathrm{~S}=1, \mathrm{Xaa}=21$

$3 \mathrm{Q}=1, \mathrm{P}=9716, \mathrm{~S}=1, \mathrm{Xaa}=21$

9739 

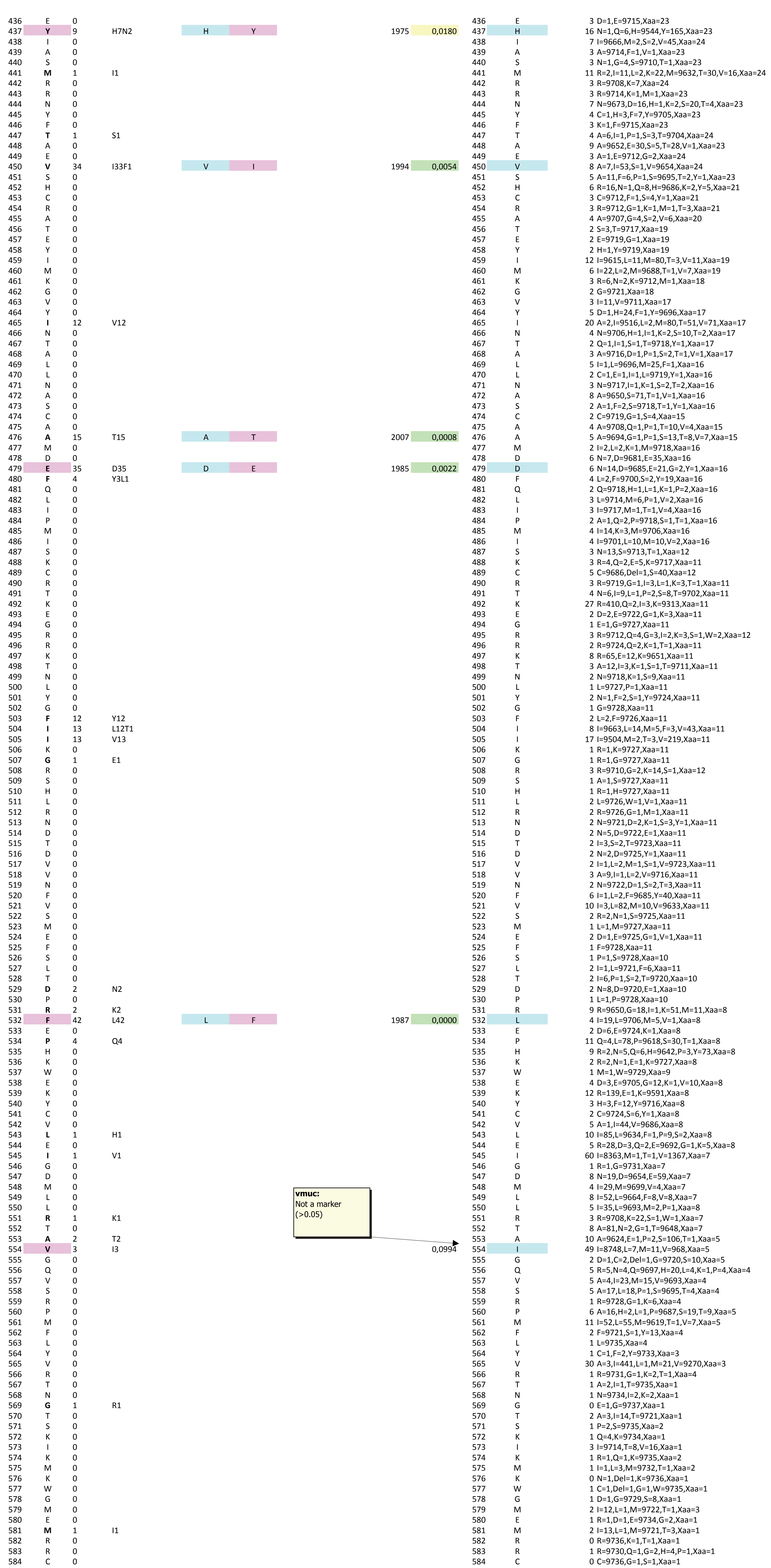

9739

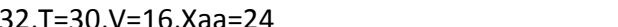

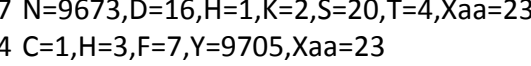

, $T=2, Y=1, X a a=23$

B $R=9712, G=1, K=1, M=1, T=3, \quad 2 a=21$

作 $=80, T=3, V=11, \mathrm{Xaa}=19$

作

作

作

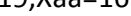

Q

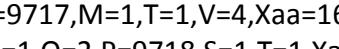

, S=1, $W=2, X a a=12$

作 $=9724, \mathrm{Xaa}=1$

$\mathrm{R}=9726, \mathrm{G}=1, \mathrm{M}=1, \mathrm{Xaa}=11$

$\mathrm{N}=9721, \mathrm{D}=2, \mathrm{~K}=1, \mathrm{~S}=3, \mathrm{Y}=1, \mathrm{Xaa}=11$

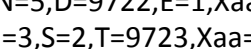

$2 \mathrm{l}=1, \mathrm{~L}=2, \mathrm{M}=1, \mathrm{~S}=1, \mathrm{~V}=9723, \mathrm{Xaa}=11$

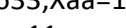

=1, V=1, Xaa $=1$

$\mathrm{P}=1, \mathrm{~S}=9728 \mathrm{Xa \textrm {a } = 1 0}$

$2 \mathrm{N=8,D=9720,E=1, \text {Xaa } = 1 0}$

$\mathrm{R}=9650, \mathrm{G}=18, \mathrm{I}=1, \mathrm{~K}=51, \mathrm{M}=11, \mathrm{Xaa}=8$

R=2, N=5 $Q=6, H=9632, P=3, Y=73, X a a$

$2 \mathrm{R}=2, \mathrm{~N}=1, \mathrm{E}=1, \mathrm{~K}=9727$, Xaa-

$\mathrm{R}=139, \mathrm{E}=1, \mathrm{~K}=9591, \mathrm{Xaa}=8$
$\mathrm{H}=3, \mathrm{~F}=12, \mathrm{Y}=9716, \mathrm{Xaa}=8$

$\mathrm{C}=9724, \mathrm{~S}=6, \mathrm{Y}=1, \mathrm{Xaa}=8$

政 $=85, \mathrm{~L}=9634, \mathrm{~F}=1, \mathrm{P}=9, \mathrm{~S}=2, \mathrm{Xaa}=8$

$60 \mathrm{l}=8363, \mathrm{M}=1, \mathrm{~T}=1, \mathrm{~V}=1367, \mathrm{Xaa}=7$

作

A A $16, \mathrm{H}=2, \mathrm{~L}=1, \mathrm{P}=9687, \mathrm{~S}=19, \mathrm{~T}=9, \mathrm{Xaa}=5$

$A=3, l=441, L=1, M=21, V=9270, \mathrm{Xaa}=3$

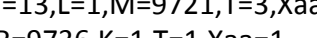

$1 \mathrm{R}=9730 \mathrm{Q}=1, \mathrm{G}=2, \mathrm{H}=4, \mathrm{P}=1, \mathrm{Xaa}=1$

C $C=9736, G=1, S=1, X a a=1$
$1\llcorner=9731, F=2, P=5, X a a=1$ 
$0 \mathrm{Q}=9736, \mathrm{P}=2, \mathrm{Xaa}=1$

$3 \mathrm{l}=9715, \mathrm{M}=2, \mathrm{~V}=21, \mathrm{Xaa}=1$

$0 \mathrm{E}=9737, \mathrm{G}=1, \mathrm{Xaa}=1$

3 $R=2, N=10, G=5, S=9719, T=1, X a a=2$

CI $=48, \mathrm{~L}=7, \mathrm{M}=9678, \mathrm{~T}=2, \mathrm{~V}=2, \mathrm{Xaa}=2$

5 Del $=1, \mathrm{l}=9691, \mathrm{~T}=1, \mathrm{~V}=45, \mathrm{Xaa}=1$

$1 \mathrm{D}=2, \mathrm{Del}=2, \mathrm{E}=9732, \mathrm{G}=1, \mathrm{~K}=1, \mathrm{X}$
$2 \mathrm{~A}=9727 \mathrm{~S}=2, \mathrm{~T}=7, \mathrm{~V}=1, \mathrm{Xaa}=2$

$2 \mathrm{D}=2, \mathrm{Q}=2, \mathrm{E}=9728, \mathrm{G}=3, \mathrm{~K}=2, \mathrm{~V}=1, \mathrm{Xaa}=1$

$1 \mathrm{~F}=5, \mathrm{P}=2, \mathrm{~S}=9730$, Xaa $=2$

$\begin{aligned} & F=2, S=9736, X a a=1 \\ & 14 A=4, I=166, F=1, T=2, V=9565, X a a=1\end{aligned}$

$14 \mathrm{~A}=4, \mathrm{l}=166, \mathrm{~F}=1, \mathrm{~T}=2, \mathrm{~V}=9565, \mathrm{Xaa}=1$
$9 \mathrm{R}=102, \mathrm{Q}=1, \mathrm{E}=1, \mathrm{~K}=9632, \mathrm{~T}=2, \mathrm{Xaa}=1$

$\mathrm{E}=9737, \mathrm{G}=1, \mathrm{Xaa}=1$

$\mathrm{R}=9, \mathrm{Q}=1, \mathrm{~K}=9728, \mathrm{Xaa}=1$

$13 \mathrm{l}=5, \mathrm{~L}=133, \mathrm{M}=9579, \mathrm{~V}=21, \mathrm{Xaa}=1$

$0 \mathrm{~S}=2, \mathrm{~T}=9736, \mathrm{Xaa}=1$

$19 \mathrm{R}=285, \mathrm{~K}=9453, \mathrm{Xaa}=1$

$7 \mathrm{~A}=2, \mathrm{D}=62, \mathrm{E}=9666, \mathrm{G}=5, \mathrm{~K}=2, \mathrm{Xaa}=2$

$1 \mathrm{~L}=4, \mathrm{~F}=9732, \mathrm{~S}=1, \mathrm{~V}=1, \mathrm{Xaa}=1$

$0 \mathrm{~F}=9737, \mathrm{~S}=1, \mathrm{Xaa}=1$

作=7,L=6,K=5,V=21, Xaa=2

13 $\mathrm{R}=4, \mathrm{~N}=98, \mathrm{~N}=2, \mathrm{~K}=9568 \mathrm{C}, \mathrm{Xaa}=1$

$4 \mathrm{~A}=6, \mathrm{~L}=10, \mathrm{P}=5, \mathrm{~S}=9702, \mathrm{~T}=15, \mathrm{Xaa}=1$

$2 \mathrm{~A}=1, \mathrm{Q}=1, \mathrm{E}=9720, \mathrm{G}=4, \mathrm{~K}=3, \mathrm{~V}=2, \mathrm{Xaa}=8$

$35 \mathrm{~A}=62, \mathrm{R}=3, \mathrm{I}=1, \mathrm{~K}=265, \mathrm{M}=129, \mathrm{~S}=2, \mathrm{~T}=9275, \mathrm{~V}=1, \mathrm{Xaa}=1$

$1 \mathrm{R}=3, \mathrm{C}=1, \mathrm{~W}=9734, \mathrm{Xaa}=1$

$\begin{array}{rl} & \\ 10 & \mathrm{I}=9621, \mathrm{M}=1, \mathrm{~T}=2, \mathrm{~V}=114, \mathrm{Xaa}=1\end{array}$

O $A=1, G=9737, X a a=1$

$1 \mathrm{D}=2, \mathrm{E}=9730, \mathrm{G}=6, \mathrm{Xaa}=1$

$\mathrm{S}=9738, \mathrm{Xaa}=1$

$\mathrm{l}=3, \mathrm{~K}=7772, \mathrm{~T}=2, \mathrm{Xaa}=1$

$A=1, R=1, E=9733, G=3, X=15, T$

$11 \mathrm{~A}=1, \mathrm{D}=80, \mathrm{E}=9625, \mathrm{G}=8, \mathrm{~K}=7, \mathrm{~V}=17$, Xaa $=1$

$24 \mathrm{R}=2, \mathrm{~N}=1, \mathrm{C}=2, \mathrm{G}=9368, \mathrm{~S}=361, \mathrm{~V}=3, \mathrm{Xaa}=2$

$2 \mathrm{~A}=1, \mathrm{~F}=4, \mathrm{P}=7, \mathrm{~S}=9726, \mathrm{Xaa}=1$

$2 \mathrm{I}=9723, \mathrm{~L}=2, \mathrm{~S}=1, \mathrm{~V}=12, \mathrm{Xaa}=1$

$0 \mathrm{R}=1, \mathrm{E}=1, \mathrm{G}=9736, \mathrm{X}$
$0 \mathrm{~K}=9738, \mathrm{Xaa}=1$

$0 \mathrm{~K}=9738, \mathrm{Xaa}=1$
$3 \mathrm{~A}=1, \mathrm{G}=2, \mathrm{l}=3, \mathrm{~L}=20, \mathrm{~V}=9712, \mathrm{Xaa}=1$

3 $\mathrm{A}=1, \mathrm{G}=2, \mathrm{l}=3, \mathrm{~L}=20, \mathrm{~V}=971$
$\mathrm{C}=9735, \mathrm{G}=2, \mathrm{Y}=1, \mathrm{Xaa}=1$

$1 \mathrm{C}=9735, \mathrm{G}=2, \mathrm{Y}=1, \mathrm{Xa}=1$
$0 \mathrm{R}=9736, \mathrm{~K}=2, \mathrm{Xaa}=1$

$3 \mathrm{~A}=30, \mathrm{~T}=9708, \mathrm{Xaa}=1$

$1 \mathrm{~L}=9734, \mathrm{M}=3, \mathrm{~K}=1, \mathrm{~W}=1$

$0 . \mathrm{l}=1, \mathrm{~L}=9736, \mathrm{M}=2$

$1 \mathrm{~A}=9728, \mathrm{G}=1, \mathrm{~S}=7, \mathrm{~T}=3$

$2 \mathrm{R}=21, \mathrm{~K}=9718$

$0 \mathrm{~F}=1, \mathrm{P}=1, \mathrm{~S}=9737$

$1 \mathrm{~A}=3, \mathrm{l}=1, \mathrm{~L}=7, \mathrm{~V}=972$

$1 \mathrm{~L}=2, \mathrm{~F}=9735, \mathrm{~S}=2$

$\mathrm{N}=9732, \mathrm{D}=2, \mathrm{~S}=2, \mathrm{~T}=3$
$\mathrm{~A}=1, \mathrm{R}=1, \mathrm{~N}=48, \mathrm{C}=8, \mathrm{G}=7, \mathrm{~S}=9674$

$6 \mathrm{I}=59, \mathrm{~L}=9676, \mathrm{M}=3, \mathrm{~S}=1$

$1 \mathrm{H}=1, \mathrm{~F}=4, \mathrm{Y}=9734$

$4 \mathrm{~A}=9697, \mathrm{~S}=39, \mathrm{~T}=3$

O $F=1, S=9738$
$30 \mathrm{~A}=21, \mathrm{P}=9261, \mathrm{~S}=452, \mathrm{~T}=5$

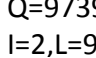

$1 \mathrm{l}=2, \mathrm{~L}=9733, \mathrm{~F}=4$

$0 \mathrm{E}=9738, X$
$0 \mathrm{G}=9739$

$L=1, F=9737, X a a=1$
$Q=1, L=1, P=1, S=973$

$1 \mathrm{~A}=9730, \mathrm{G}=1, \mathrm{~L}=1, \mathrm{P}=1, \mathrm{~S}=6$

0 Del $=1, \mathrm{E}=9738$

$2 A=15, L=2, S=9722$

$0 \mathrm{R}=9737, \mathrm{Q}=1, \mathrm{~K}=1$

$3 \mathrm{R}=28, \mathrm{E}=1, \mathrm{~K}=9709, \mathrm{Xaa}=1$
$\mathrm{~L}=19, \mathrm{~L}=9638, \mathrm{M}=77, \mathrm{~F}=5$

$9 \mathrm{l}=19, \mathrm{~L}=9638, \mathrm{M}=77, \mathrm{~F}=5$

$1 \mathrm{l}=2, \mathrm{~L}=9729, \mathrm{~F}=7, \mathrm{~T}=1$

$1 \mathrm{~L}=9734, \mathrm{~F}=3, \mathrm{~S}=1, \mathrm{Xaa}=1$

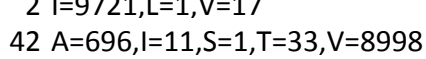

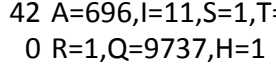

$0 R=1, Q=9737, H$
$0 A=9739$

93 Del=3097, $\mathrm{l}=3, \mathrm{~L}=6616, \mathrm{~F}=21, \mathrm{~V}=1, \mathrm{Xaa}=1$

$4 \mathrm{R}=9704, \mathrm{G}=9, \mathrm{~K}=17, \mathrm{M}=9$

1 A $=1, D=9735, G=3$

$13 \mathrm{~N}=9600, \mathrm{D}=1, \mathrm{G}=1, \mathrm{H}=7, \mathrm{~K}=25, \mathrm{~S}=94, \mathrm{~T}=2, \mathrm{Y}=8, \mathrm{X}$ aa $=1$

$0 \mathrm{~L}=9738, \mathrm{P}=1$

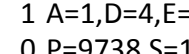

O $P=9738, S=1$
$0 R=1, G=9737, W=1$

$1 \mathrm{~A}=1, \mathrm{~S}=4, \mathrm{~T}=9733$, Xaa $=$

$0 \mathrm{~L}=1, \mathrm{~F}=9738$

$3 \mathrm{~N}=24, \mathrm{D}=9712, \mathrm{G}=1, \mathrm{H}=1, \mathrm{Y}=1$

$10 \mathrm{I}=120, \mathrm{~L}=9615, \mathrm{~F}=4$
$18 \mathrm{~A}=6, \mathrm{R}=14, \mathrm{E}=210, \mathrm{G}=9507, \mathrm{~W}=2$

$0 \mathrm{G}=9739$

$1 \mathrm{Q}=1, \mathrm{~L}=9730, \mathrm{~V}=8$

$9 \mathrm{D}=8, \mathrm{E}=9645, \mathrm{G}=82, \mathrm{~K}=2, \mathrm{~V}=2$

$9 \mathrm{D}=8, \mathrm{E}=9645, \mathrm{G}=82, \mathrm{~K}=2, \mathrm{C}$
$1 \mathrm{~A}=9728, \mathrm{P}=1, \mathrm{~S}=10$

$1 \mathrm{~A}=9728, \mathrm{P}=1, \mathrm{~S}=10$
$4 \mathrm{I}=9704, \mathrm{M}=2, \mathrm{~T}=4, \mathrm{~V}=29$

$0 \mathrm{E}=9738, \mathrm{G}=1$

$1 \mathrm{E}=9735, \mathrm{G}=3, \mathrm{~K}=1$

$1 \mathrm{R}=1, \mathrm{C}=9735, \mathrm{G}=2, \mathrm{Y}=1$

$1 \mathrm{~L}=9728, \mathrm{M}=5, \mathrm{P}=1, \mathrm{~V}=5$

$3 \mathrm{I}=9716, \mathrm{~L}=1, \mathrm{~T}=1, \mathrm{~V}=21$
$2 \mathrm{~N}=9718 \mathrm{~K}=1, \mathrm{~S}=\mathrm{V}, \mathrm{Xaa}=1$

$\mathrm{N}=21, \mathrm{D}=9714, \mathrm{E}=1, \mathrm{G}=1, \mathrm{Xaa}=2$

$1 \mathrm{~L}=1, \mathrm{P}=9735, \mathrm{~S}=3$

$\mathrm{R}=1, \mathrm{~L}=1, \mathrm{~W}=9736$, Xaa $=1$

$1 \mathrm{~A}=2, \mathrm{G}=2, \mathrm{l}=1, \mathrm{~L}=1, \mathrm{~V}=9733$

$0 \mathrm{C}=1, \mathrm{~L}=9737, \mathrm{~F}=1$

$1 \mathrm{l}=2, \mathrm{~L}=9735, \mathrm{P}=1, \mathrm{~V}=1$
$1 \mathrm{~N}=9732, \mathrm{D}=1, \mathrm{l}=1, \mathrm{~K}=1, \mathrm{~S}=4$

1 $A=9738, V=1$
$F=3, P=3, S=9729, Y=4$

$1 \mathrm{C}=1, \mathrm{G}=3, \mathrm{~L}=1, \mathrm{~W}=9731, \mathrm{Xaa}=3$

$4 \mathrm{~L}=30, \mathrm{~F}=9705, \mathrm{~S}=3, \mathrm{~V}=1$

$0 \mathrm{~N}=9736, \mathrm{l}=1, \mathrm{~S}=1, \mathrm{X}=\mathrm{Xa}=1$

$1 \mathrm{~F}=5, \mathrm{P}=1, \mathrm{~S}=9733$

$1 \mathrm{~L}=4, \mathrm{~F}=9733, \mathrm{~S}=2$

$3 \mathrm{Q}=1, \mathrm{l}=6, \mathrm{~L}=9714, \mathrm{~F}=17, \mathrm{P}=1$

$60 \mathrm{~A}=764, \mathrm{R}=1, \mathrm{l}=234, \mathrm{~K}=3, \mathrm{M}=20, \mathrm{P}=4, \mathrm{~T}=8705, \mathrm{~V}=6, \mathrm{Xaa}=2$

$1 \mathrm{R}=2, \mathrm{~N}=1, \mathrm{Q}=1, \mathrm{H}=9734, \mathrm{Y}=1$

$4 \mathrm{~A}=9699, \mathrm{~S}=1, \mathrm{~T}=1, \mathrm{~V}=38$

$1 \mathrm{R}=2, \mathrm{E}=1, \mathrm{G}=1, \mathrm{~L}=9734, \mathrm{~V}=1$ 


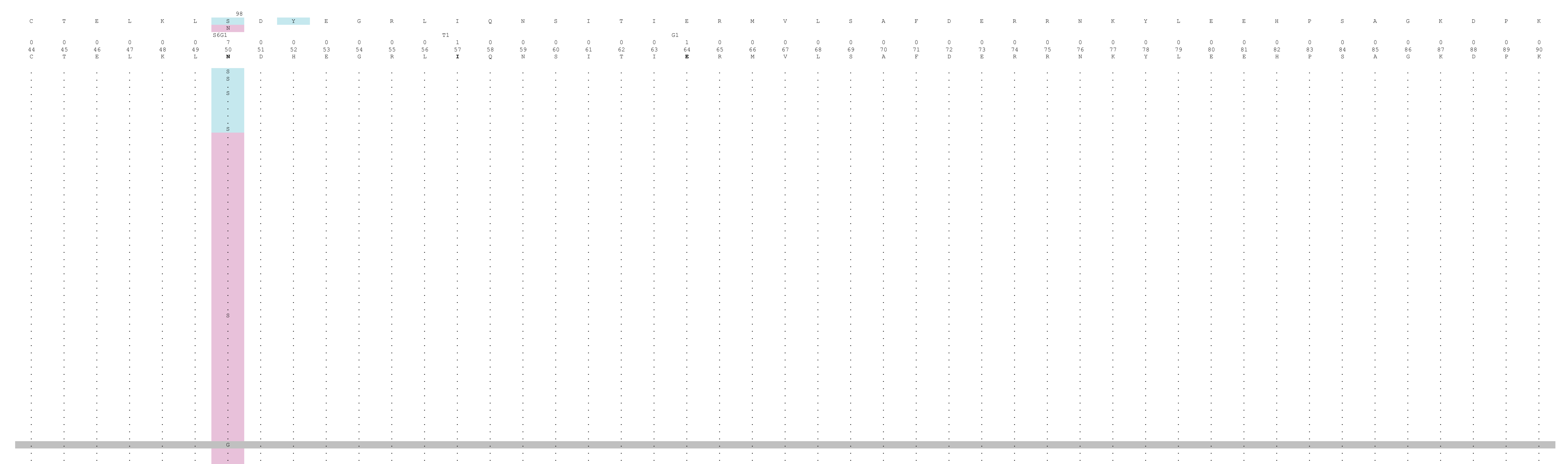



<smiles>CC(C)(C)C</smiles> 




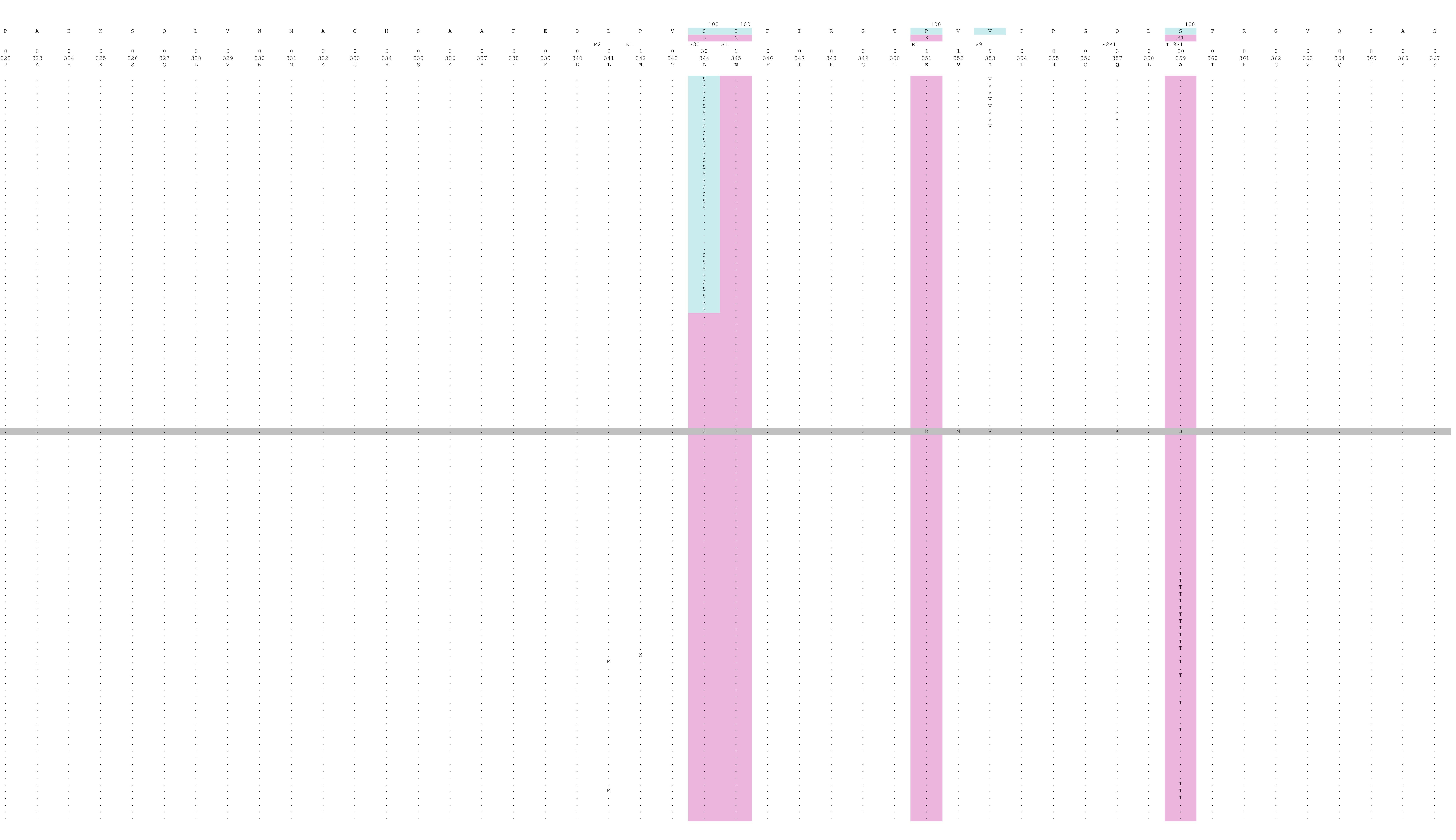





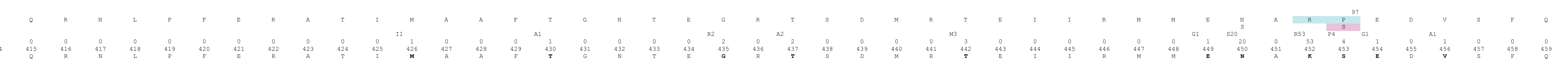

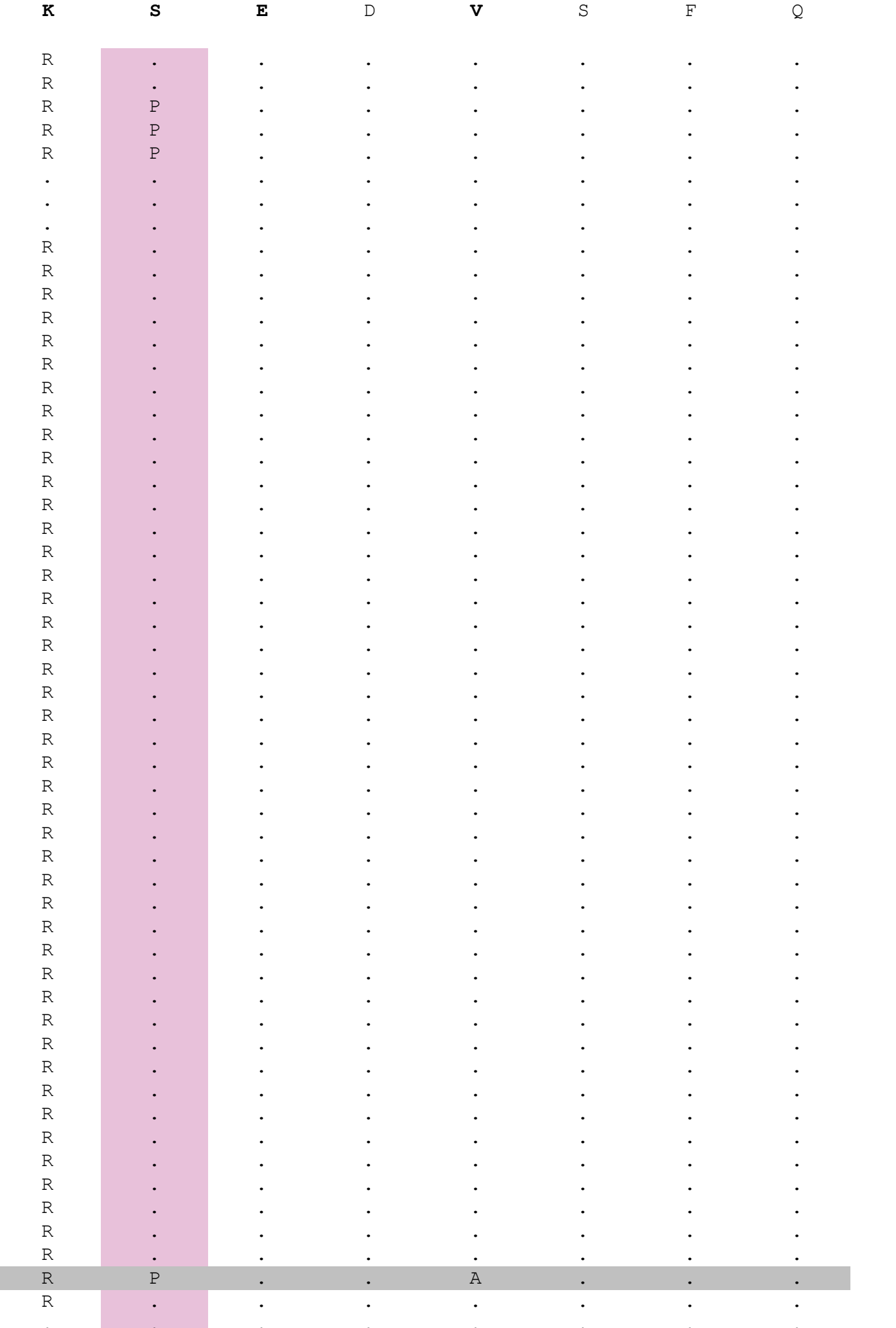




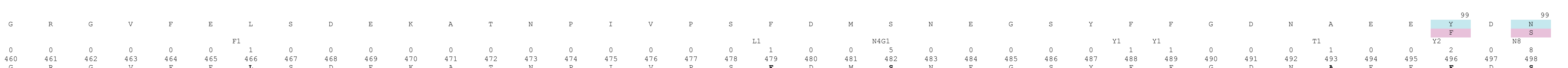


$0 \mathrm{~A}=1, \mathrm{I}=2, \mathrm{~T}=9236$

1
1 $\mathrm{Q}=9233, \mathrm{H}=5, \mathrm{Xaa}=1$
$\mathrm{R}=9237, \mathrm{~S}=1, \mathrm{~T}=1$

O $P=1, T=9237$, $\mathrm{Xaa}=$

$\mathrm{R}=9236, \mathrm{G}=3$
$\mathrm{O} \mathrm{A}=9237, \mathrm{G}=1, \mathrm{P}=1$
$1 \mathrm{H}=2, \mathrm{I}=2, \mathrm{~L}=9233, \mathrm{~F}=1, \mathrm{~V}=1$

$0 \mathrm{~A}=1, I=1, \mathrm{~V}=$
$0 \mathrm{R}=9239$

$5 \mathrm{~A}=16, \mathrm{P}=1, \mathrm{~S}=173, \mathrm{~T}=9049$

O $\mathrm{G}=9238, \mathrm{Xaa}=1$

$0 \mathrm{~L}=1, \mathrm{M}=9237, \mathrm{~V}=1$
$0 \mathrm{D}=9237, \mathrm{G}=2$

$0 \mathrm{D}=9237, \mathrm{G}=2$
$\mathrm{O} \mathrm{A}=1, \mathrm{P}=9237, \mathrm{I}=1$

$1 \mathrm{R}=9233, \mathrm{I}=1, \mathrm{~K}=2, \mathrm{M}=1, \mathrm{~T}=1, \mathrm{Xaa}=1$

I $\mathrm{M}=9231, \mathrm{~T}=6, \mathrm{Xaa}=2$

$1 \mathrm{R}=1, \mathrm{C}=9235, \mathrm{G}=1, \mathrm{Xaa}=2$
$1 \mathrm{C}=1, \mathrm{~F}=1, \mathrm{P}=1, \mathrm{~S}=9234, \mathrm{Xaa}=2$

$1 \mathrm{I}=1, \mathrm{~L}=9235, \mathrm{M}=1, \mathrm{Xaa}=2$

$\mathrm{Q}=9233, \mathrm{H}=1, \mathrm{~L}=1, \mathrm{~K}=1, \mathrm{P}=1, \mathrm{Xaa}=2$

$1 \mathrm{~A}=1, \mathrm{R}=1, \mathrm{G}=9235, \mathrm{Xaa}=2$

$\mathrm{A}=1, \mathrm{~N}=1, \mathrm{~S}=1, \mathrm{~T}=9234, \mathrm{Xaa}=2$

$\mathrm{P}=9236, \mathrm{Xaa}=3$

$\mathrm{R}=9234, \mathrm{~K}=1, \mathrm{~T}=1, \mathrm{Xaa}=3$

$\mathrm{R}=9236, \mathrm{~K}=1, \mathrm{Xaa}=2$
$\mathrm{P}=1, \mathrm{~S}=9235, \mathrm{Xaa}=3$

$\mathrm{R}=1, \mathrm{G}=9236, \mathrm{Xaa}=2$

$\mathrm{A}=9233, \mathrm{D}=1, \mathrm{~F}=1, \mathrm{~S}=1, \mathrm{~V}=1, \mathrm{Xaa}=2$

1, Хaa $=2$

A $=1, \mathrm{I}=218, \mathrm{~L}=1, \mathrm{M}=42, \mathrm{~V}=8974, \mathrm{Xaa}=3$

$\mathrm{R}=1, \mathrm{~K}=9234, \mathrm{Xaa}=4$

$24 \mathrm{I}=361, \mathrm{~V}=8875, \mathrm{Xaa}=3$

$1 \mathrm{R}=3, \mathrm{G}=9233$, $\mathrm{Xaa}=3$

$1 \mathrm{~A}=1, \mathrm{Q}=1, \mathrm{P}=1, \mathrm{~T}=9233, \mathrm{Xaa}=3$

$\mathrm{A}=68, \mathrm{G}=2, \mathrm{I}=5, \mathrm{~T}=2, \mathrm{~V}=9159, \mathrm{Xaa}=3$

$\mathrm{A} \mathrm{A}=5, \mathrm{M}=9229, \mathrm{~V}=2, \mathrm{xaa}=3$
$\mathrm{I}=5, \mathrm{~s}$

$=9235, \mathrm{Xaa}=4$

$2 \mathrm{I}=14, \mathrm{~L}=9220, \mathrm{M}=1, \mathrm{~V}=1, \mathrm{Xaa}=3$

$20 \mathrm{~N}=1, \mathrm{I}=8975, \mathrm{~F}=1, \mathrm{~T}=7, \mathrm{~V}=252, \mathrm{Xaa}=3$

$\mathrm{I}=9178, \mathrm{~T}=3, \mathrm{~V}=55, \mathrm{X}=3 \mathrm{a}$

$\mathrm{R}=12, \mathrm{~N}=2, \mathrm{~K}=9219, \mathrm{~S}=1, \mathrm{~T}=2, \mathrm{Xa} a=3$

$\mathrm{R}=9233, \mathrm{Q}=2, \mathrm{G}=1, \mathrm{Xaa}=3$

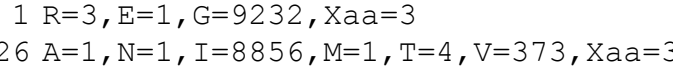

$1 \mathrm{~N}=9231, \mathrm{D}=1, \mathrm{H}=1, \mathrm{~S}=2, \mathrm{~T}=1, \mathrm{Xaa}=3$

$\mathrm{R}=9231, \mathrm{Q}=1, \mathrm{P}=3, \mathrm{~W}=1, \mathrm{Xaa}=3$

$1 \mathrm{~N}=9234, \mathrm{~K}=1, \mathrm{~S}=1, \mathrm{Xaa}=3$

$1 \mathrm{~F}=9233, \mathrm{Xaa}=6$

$1 \mathrm{~W}=9234, \mathrm{Xaa}=5$

$1 \mathrm{R}=9233, \mathrm{G}=1$, Xaa -5

$1 \mathrm{G}=9234, \mathrm{Xaa}=5$

$4 \mathrm{~N}=9208, \mathrm{D}=2, \mathrm{~S}=14, \mathrm{P}=1, \mathrm{Xaa}=5$

$1 \mathrm{E}=2, \mathrm{G}=9232, \mathrm{Xaa}=5$

$1 \mathrm{R}=9233, \mathrm{P}=1, \mathrm{xaa}=5$

$\mathrm{R}=9148, \mathrm{~L}=1, \mathrm{~K}=85, \mathrm{Xaa}=5$

$3 \mathrm{R}=9214, \mathrm{G}=1, \mathrm{~K}=16, \mathrm{M}=2, \mathrm{~W}=1, \mathrm{X} a \mathrm{a}=5$

$27 \mathrm{~A}=2, \mathrm{~N}=3, \mathrm{I}=8909, \mathrm{~L}=1, \mathrm{M}=5, \mathrm{~F}=1, \mathrm{~T}=222, \mathrm{~V}=91, \mathrm{Xaa}=5$

$1 \mathrm{~A}=9230, \mathrm{G}=1, \mathrm{P}=1, \mathrm{~T}=2, \mathrm{Xaa}=5$

$1 \mathrm{C}=1, \mathrm{Y}=9233, \mathrm{Xaa}=5$

$\mathrm{D}=8, \mathrm{E}=9224, \mathrm{~K}=1, \mathrm{Xaa}=$

$\mathrm{R}=9233, \mathrm{G}=1, \mathrm{Xaa}=5$
$\mathrm{I}=2, \mathrm{M}=9232, \mathrm{Xaa}=5$

$1 \mathrm{C}=9232, \mathrm{~S}=1, \mathrm{Y}=1, \mathrm{Xaa}=5$

$\mathrm{N}=9232, \mathrm{~K}=1, \mathrm{~T}=1, \mathrm{Xaa}=5$

$\mathrm{I}=9229, \mathrm{~V}=5, \mathrm{Xaa}=5$
$\mathrm{I}=9233, \mathrm{P}=1, \mathrm{Xaa}=5$

$1 \mathrm{~L}=9233, \mathrm{P}=1, \mathrm{Xaa}=5$
$\mathrm{~K}=9234, \mathrm{Xaa}=5$

$\mathrm{K}=2234, \mathrm{Xa}=5$
$\mathrm{R}=2, \mathrm{G}=9232, \mathrm{Xaa}=5$

$1 \mathrm{~K}=9232, \mathrm{~T}=1, \mathrm{Xaa}=6$

$\mathrm{I}=2, \mathrm{~L}=14, \mathrm{~F}=9216, \mathrm{~S}=2, \mathrm{Xaa}=5$

$\mathrm{R}=2, \mathrm{Q}=9229, \mathrm{P}=3, \mathrm{Xaa}=5$

$3 \mathrm{~A}=2, \mathrm{I}=12, \mathrm{~T}=9219, \mathrm{Xa} a=$

1 A $=9234, x$ aa $=5$

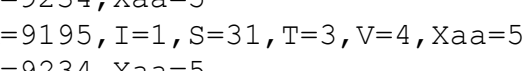

$\mathrm{A}=9208, \mathrm{G}=15, \mathrm{~S}=1, \mathrm{~T}=7, \mathrm{~V}=3, \mathrm{Xaa}=5$

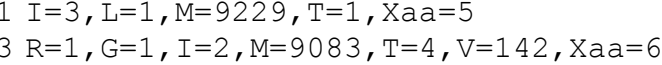

1. $\mathrm{R}=1, \mathrm{~A}=1, \mathrm{I}=2, \mathrm{M}=9083, \mathrm{~T}=4, \mathrm{~V}=142 \mathrm{~N}=1, \mathrm{D}=9231, \mathrm{I}=1, \mathrm{Xa}=5$

$\mathrm{Q}=9232, \mathrm{H}=1, \mathrm{~K}=1, \mathrm{Xaa}=5$

$\mathrm{V}=9229, \mathrm{xaa}=5$

$\mathrm{R}=9231, \mathrm{Q}=2, \mathrm{~K}=1, \mathrm{Xaa}=5$

$\mathrm{E}=9233, \mathrm{~K}=1, \mathrm{Xaa}=5$

$\mathrm{N}=1, \mathrm{G}=19, \mathrm{~S}=9214, \mathrm{Xaa}=5$

$9 \mathrm{~N}=9149, \mathrm{D}=1, \mathrm{~K}=1, \mathrm{H}=1, \mathrm{X}=79, \mathrm{~T}=3, \mathrm{Xa}=5$

$1 \mathrm{P}=9233, \mathrm{~T}=1, \mathrm{Xaa}=5$
$1 \mathrm{R}=2, \mathrm{E}=2, \mathrm{G}=9229, \mathrm{~V}=1, \mathrm{Xaa}=5$

$3 \mathrm{~A}=9208, \mathrm{~V}=26, \mathrm{Xaa}=5$

$\mathrm{E}=9234, \mathrm{Xaa}=5$

$\mathrm{N}=1, \mathrm{D}=9229, \mathrm{E}=1, \mathrm{G}=2, \mathrm{H}=1, \mathrm{Xaa}=5$

$1 \mathrm{I}=2, \mathrm{~L}=9222, \mathrm{I}=1, \mathrm{~V}=2, \mathrm{Xa}=5$
$\mathrm{~A}=1 \mathrm{I}=9151, \mathrm{~L}=3, \mathrm{~S}=1, \mathrm{~T}=75, \mathrm{~V}=3, \mathrm{Xaa}=5$

$\mathrm{R}=2, \mathrm{Q}=1, \mathrm{I}=2, \mathrm{I}=9224, \mathrm{P}=1, \mathrm{~S}=4, \mathrm{Xaa}=5$

$\mathrm{A}=9226, \mathrm{~T}=7, \mathrm{Xaa}=6$

$\mathrm{R}=9234, \mathrm{Xaa}=5$

$\mathrm{C}=1, \mathrm{~S}=9232, \mathrm{Xaa}=6$
$\mathrm{~A}=9232, \mathrm{P}=1, \mathrm{Xaa}=6$

$1 \mathrm{~A}=9232, \mathrm{P}=1, \mathrm{Xa}=6$
$\mathrm{I}=1, \mathrm{~L}=9231, \mathrm{P}=2, \mathrm{Xaa}=5$

I $=9216, \mathrm{~L}=1, \mathrm{~V}=15, \mathrm{Xa}=5$

$\mathrm{Q}=1, \mathrm{~L}=9227, \mathrm{M}=1, \mathrm{P}=2, \mathrm{~V}=2, \mathrm{xaa}=6$

$1 \mathrm{R}=9232, \mathrm{~K}=1, \mathrm{Xaa}=6$

$1 \mathrm{R}=2, \mathrm{E}=2, \mathrm{G}=9229$, $\mathrm{Xaa}=6$

$10 \mathrm{~A}=98, \mathrm{P}=2, \mathrm{~S}=9131, \mathrm{~T}=2, \mathrm{Xaa}=6 \mathrm{x}$

$\mathrm{A}=2, \mathrm{I}=78, \mathrm{~L}=2, \mathrm{M}=21, \mathrm{~V}=9130$,
$\mathrm{A}=9229, \mathrm{G}=1, \mathrm{P}=2, \mathrm{~S}=1, \mathrm{Xaa}=6$

$\mathrm{H}=9232, \mathrm{~L}=1, \mathrm{Xaa}=6$

$\mathrm{K}=9233, \mathrm{Xaa}=6$

1 $S=9233$, xaa $=6$

$2 \mathrm{C}=9225, \mathrm{~S}=5, \mathrm{~W}=1, \mathrm{Y}=1, \mathrm{Xaa}=7$

$\mathrm{L}=9226, \mathrm{M}=4, \mathrm{P}=1, \mathrm{~W}=1, \mathrm{Xaa}=7$
$\mathrm{~A}=1, \mathrm{H}=2, \mathrm{P}=9228, \mathrm{~S}=1, \mathrm{Xaa}=7$

$\mathrm{A}=9226, \mathrm{D}=1, \mathrm{~S}=2, \mathrm{~T}=2, \mathrm{~V}=1, \mathrm{Xaa}=7$

$1 \mathrm{R}=1, \mathrm{C}=9228, \mathrm{G}=2, \mathrm{Xaa}=8$
$\mathrm{~A}=3, \mathrm{G}=1, \mathrm{I}=31, \mathrm{M}=5, \mathrm{~V}=9191, \mathrm{Xaa}=8$

$\mathrm{N}=1, \mathrm{H}=1, \mathrm{~F}=1, \mathrm{~S}=1, \mathrm{Y}=9227, \mathrm{Xaa}=8$

$3 \mathrm{I}=15, \mathrm{I}=9213, \mathrm{P}=4, \mathrm{Xa}=7$

$\mathrm{A}=9226, \mathrm{~T}=4, \mathrm{~V}=2, \mathrm{Xaa}=7$

$2 \mathrm{~A}=1, \mathrm{I}=6, \mathrm{~L}=1, \mathrm{~V}=9224, \mathrm{Xaa}=7$ 


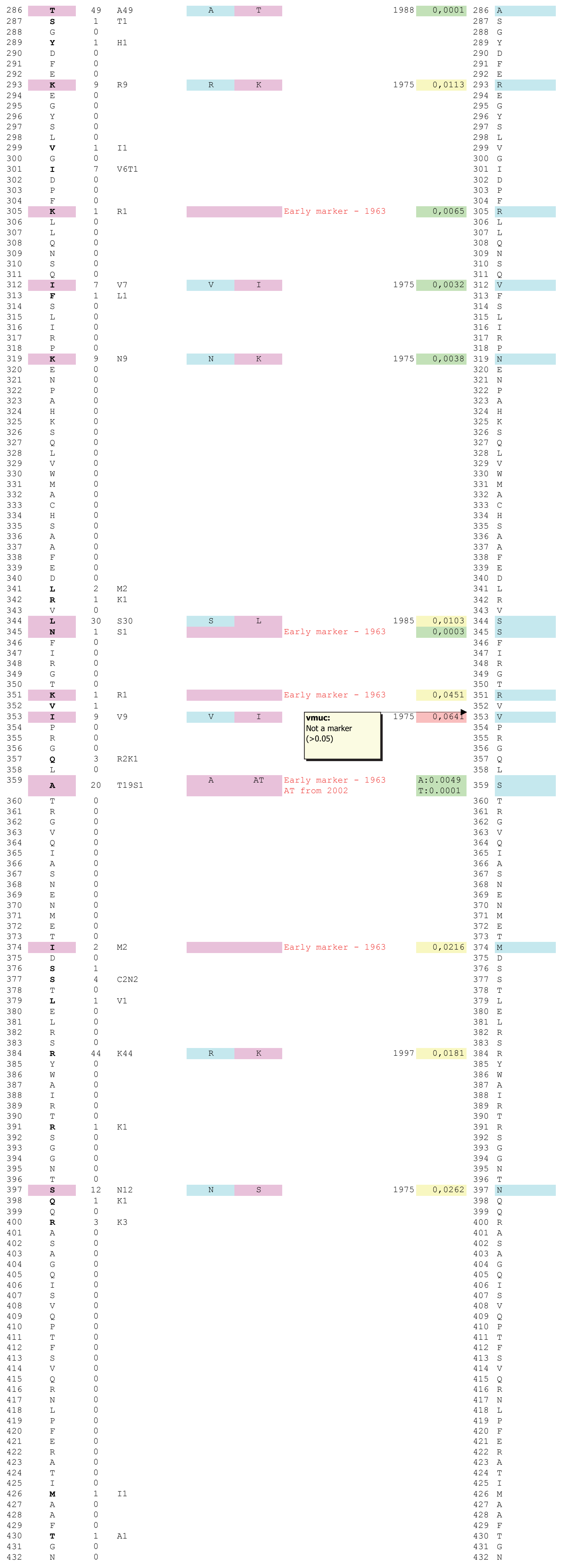

$6 \mathrm{~A}=9181, \mathrm{~L}=2, \mathrm{P}=3, \mathrm{~S}=1, \mathrm{~T}=1, \mathrm{~V}=44, \mathrm{Xaa}=7$
$11 \mathrm{~N}=16, \mathrm{G}=96, \mathrm{I}=1, \mathrm{~S}=9118, \mathrm{~T}=1, \mathrm{Xa}=7$

$1 \mathrm{~A}=2, \mathrm{R}=1, \mathrm{G}=9230, \mathrm{Xaa}=6$
$6 \mathrm{H}=38, \mathrm{~F}=11, \mathrm{Y}=9184, \mathrm{Xa}$

$10 \mathrm{~A}=1, \mathrm{~N}=67, \mathrm{D}=9142, \mathrm{E}=14, \mathrm{G}=1, \mathrm{H}=1, \mathrm{~S}=3, \mathrm{~V}=1, \mathrm{Xaa}=8$

政,

2 $\mathrm{A}=2, \mathrm{D}=3, \mathrm{G}=9225, \mathrm{~K}=1, \mathrm{~K}, \mathrm{Kaa}=6 \mathrm{x}, \mathrm{Xaa}=6$

$1 \mathrm{R}=4, \mathrm{G}=9229$, $\mathrm{Xaa}=6$

$\mathrm{L}=9231, \mathrm{P}=1, \mathrm{~S}=1, \mathrm{~V}=1, \mathrm{Xaa}=5$

$2, \mathrm{~V}=9232, \mathrm{Xaa}=5$

$5 \mathrm{R}=6, \mathrm{~N}=1, \mathrm{I}=9079, \mathrm{~L}=18, \mathrm{~K}=1, \mathrm{M}=12, \mathrm{~F}=4, \mathrm{~V}=113, \mathrm{Xaa}=5$

2 $\mathrm{A}=2, \mathrm{~N}=1, \mathrm{D}=9223, \mathrm{E}=2, \mathrm{G}=3, \mathrm{Xaa}=8$

$2 \mathrm{~L}=1, \mathrm{P}=9226, \mathrm{~S}=7, \mathrm{Xaa}=5$
$2 \mathrm{~L}=1, \mathrm{~F}=9227, \mathrm{~S}=3, \mathrm{Y}=3, \mathrm{Xaa}=5$

$8 \mathrm{R}=9165, \mathrm{H}=7, \mathrm{~K}=60, \mathrm{~S}=2, \mathrm{Xa} a=5$

$2 \mathrm{~L}=9226, \mathrm{M}=5, \mathrm{P}=1, \mathrm{~V}=2, \mathrm{Xa} a=5$

$1 \mathrm{~L}=9233, \mathrm{~F}=1, \mathrm{Xaa}=5$
$4 \mathrm{Q}=9207, \mathrm{~K}=27, \mathrm{Xaa}=5$

$4 \mathrm{Q}=9207, \mathrm{~K}=27, \mathrm{Xaa}=5$
$2 \mathrm{~A}=1, \mathrm{~N}=9224, \mathrm{D}=1, \mathrm{~K}=4, \mathrm{~S}=2, \mathrm{~T}=2, \mathrm{Xaa}=5$

$2 \mathrm{~A}=1, \mathrm{~N}=9224, \mathrm{D}=1, \mathrm{~K}=4, \mathrm{~S}=2, \mathrm{~T}=2, \mathrm{Xa}$
$4 \mathrm{~N}=1, \mathrm{G}=2, \mathrm{~S}=9205, \mathrm{~T}=23, \mathrm{~V}=3, \mathrm{xa}=5$

$4 \mathrm{~N} R=1, \mathrm{G}=2, \mathrm{~S}=92 \mathrm{Xa}, \mathrm{T}=23, \mathrm{~V}$
$1 \mathrm{R}=1, \mathrm{Q}=9233, \mathrm{Xaa}=5$

$4 \mathrm{~A}=1, \mathrm{I}=30, \mathrm{~V}=9203, \mathrm{Xaa}=5$

$9 \mathrm{I}=8, \mathrm{~L}=53, \mathrm{~F}=9151, \mathrm{~S}=14, \mathrm{Y}=2, \mathrm{~V}=6, \mathrm{Xa} a=5$

$1 \mathrm{C}=2, \mathrm{~S}=9232, \mathrm{Xaa}=5$

$4 \mathrm{I}=31, \mathrm{~L}=9202, \mathrm{P}=1, \mathrm{Xaa}=5$
$5 \mathrm{I}=9194, \mathrm{~L}=1, \mathrm{M}=7, \mathrm{~T}=1, \mathrm{~V}=31, \mathrm{Xaa}=5$

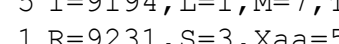

$18 \mathrm{Q}=1, \mathrm{~L}=4, \mathrm{~K}=1, \mathrm{P}=9017, \mathrm{~S}=207, \mathrm{~T}=4, \mathrm{xaa}=5$

$6 \mathrm{~N}=9182, \mathrm{D}=1, \mathrm{I}=1, \mathrm{~K}=35, \mathrm{~S}=12, \mathrm{~T}=3, \mathrm{Xa} a=5$

$1 \mathrm{D}=1, \mathrm{E}=9231, \mathrm{G}=1, \mathrm{~K}=1, \mathrm{Xaa}=5$

3 $\mathrm{N}=9212, \mathrm{I}=1, \mathrm{~K}=4, \mathrm{~S}=15, \mathrm{~T}=1, \mathrm{Y}=1, \mathrm{Xaa}=5$

3) $\mathrm{A}=2, \mathrm{Q}=1, \mathrm{P}, \mathrm{K}=4, \mathrm{~S}, \mathrm{~S}=15, \mathrm{I}=1, \mathrm{Xa}=5$

$1 \mathrm{Q}=1, \mathrm{H}=9232, \mathrm{Xaa}=6$

$1 \mathrm{R}=1, \mathrm{Q}=1, \mathrm{~K}=9231, \mathrm{Xaa}=6$
$2 \mathrm{G}=9, \mathrm{I}=1, \mathrm{~S}=9224, \mathrm{Xaa}=5$

$1 \mathrm{R}=1, \mathrm{Q}=9232, \mathrm{P}=1$, $\mathrm{Xaa}=5$

$11 \mathrm{~A}=1, \mathrm{E}=1, \mathrm{I}=110, \mathrm{M}=2, \mathrm{~V}=9120, \mathrm{Xa} a=5$

$1 \mathrm{G}=1, \mathrm{~W}=9233, \mathrm{Xaa}=5$
$5 \mathrm{I}=21, \mathrm{~L}=7, \mathrm{M}=9200, \mathrm{~T}=1, \mathrm{~V}=5, \mathrm{Xaa}=5$

$1 \mathrm{~A}=9232, \mathrm{~T}=2, \mathrm{Xaa}=5$
$1 \mathrm{C}=9233, \mathrm{~S}=1, \mathrm{Xaa}=5$

$1 \mathrm{C} C=9233, \mathrm{~S}=1, \mathrm{Xaa}=5$
$1 \mathrm{R}=1, \mathrm{~N}=2, \mathrm{H}=9229, \mathrm{P}=1, \mathrm{Y}=1, \mathrm{Xaa}=5$

$2 \mathrm{~A}=9224, \mathrm{P}=2, \mathrm{~S}=3, \mathrm{~T}=3, \mathrm{~V}=2, \mathrm{Xaa}=8$

$2 \mathrm{~A}=9226, \mathrm{~S}=1, \mathrm{~T}=3, \mathrm{~V}=5, \mathrm{Xaa}=4$

$1 \mathrm{~F}=9231, \mathrm{~S}=1, \mathrm{Y}=2, \mathrm{Xaa}=5$

$1 \mathrm{~N}=2, \mathrm{D}=9231, \mathrm{G}=1, \mathrm{H}=1, \mathrm{Y}=1, \mathrm{Xaa}=3$

$1 \mathrm{R}=923, \mathrm{G}=2, \mathrm{~K}=1, \mathrm{~S}=1$

$3 \mathrm{~A}=1, \mathrm{I}=15, \mathrm{~L}=1, \mathrm{M}=6, \mathrm{~V}=921$

$9 \mathrm{~A}=1, \mathrm{~L}=95, \mathrm{~F}=1, \mathrm{~S}=9136, \mathrm{~T}=3, \mathrm{Xaa}=3$

$2 \mathrm{~N}=3, \mathrm{G}=8, \mathrm{~S}=9224, \mathrm{~T}=1, \mathrm{Xaa}=3$

2 $\mathrm{L}=13, \mathrm{~F}=9221, \mathrm{Xaa}=5$
$3 \mathrm{I}=9214, \mathrm{~L}=2, \mathrm{~V}=20, \mathrm{xaa}=3$

$1 \mathrm{R}=9233, \mathrm{G}=1, \mathrm{~K}=2, \mathrm{Xaa}=3$

$1 \mathrm{R}=1, \mathrm{G}=9235$, Xaa $=3$

$37 \mathrm{~A}=492, \mathrm{I}=23, \mathrm{~K}=30, \mathrm{M}=2, \mathrm{P}=1, \mathrm{~S}=7, \mathrm{~T}=8681$, $\mathrm{Xaa}=3$

$27 \mathrm{R}=8816, \mathrm{G}=1, \mathrm{I}=2, \mathrm{~K}=417$, $\mathrm{Xaa}=3$

$27 \mathrm{~A}=7, \mathrm{I}=93, \mathrm{~L}=39, \mathrm{M}=175, \mathrm{~V}=8921, \mathrm{Xaa}=4$

$51 \mathrm{~A}=52, \mathrm{I}=592, \mathrm{~L}=124, \mathrm{~F}=6, \mathrm{~S}=1, \mathrm{~V}=8461$, $\mathrm{Xaa}=3$

$1 \mathrm{P}=9235, \mathrm{~S}=1, \mathrm{Xaa}=3$
$\mathrm{O} \mathrm{R}=9236, \mathrm{Xaa}=3$

$1 \mathrm{R}=3, \mathrm{E}=1, \mathrm{G}=9232, \mathrm{Xaa}=3$

$6 \mathrm{R}=4, \mathrm{Q}=9178, \mathrm{~K}=53, \mathrm{~T}=1, \mathrm{Xaa}=3$
$2 \mathrm{Q}=1, \mathrm{~L}=9223, \mathrm{M}=10, \mathrm{~T}=1, \mathrm{Y}=1, \mathrm{Xaa}=3$

$6 \mathrm{~A}=45, \mathrm{C}=1, \mathrm{P}=1, \mathrm{~S}=9186, \mathrm{~T}=1, \mathrm{Y}=1, \mathrm{~V}=1, \mathrm{Xaa}=3$

$1 \mathrm{~A}=1, \mathrm{P}=2, \mathrm{~T}=9232, \mathrm{Xaa}=4$

1 $\mathrm{R}=9232, \mathrm{G}=1, \mathrm{I}=1, \mathrm{~K}=2, \mathrm{Xaa}=3$

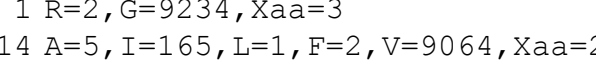

$1 \mathrm{Q}=9233, \mathrm{E}=1, \mathrm{~L}=2, \mathrm{~K}=1, \mathrm{Xaa}=2$

$1 \mathrm{~A}=9232, \mathrm{~S}=6, \mathrm{Xaa}=1$

1 $\mathrm{L}=1, \mathrm{~F}=1, \mathrm{P}=1, \mathrm{~S}=9234, \mathrm{~W}=1, \mathrm{Xaa}=$

$3 \mathrm{~N}=9210, \mathrm{~K}=3, \mathrm{~F}=1, \mathrm{~S}=24, \mathrm{X} a \mathrm{a}=1$
$1 \mathrm{E}=9235, \mathrm{G}=1, \mathrm{~K}=2, \mathrm{Xaa}=1$

$1 \mathrm{E}=9235, \mathrm{G}=1, \mathrm{~K}=2, \mathrm{Xaa}=1$
$3 \mathrm{~N}=9212, \mathrm{H}=2, \mathrm{~S}=24, \mathrm{Xaa}=1$

$40 \mathrm{~A}=1, \mathrm{I}=91, \mathrm{~L}=5, \mathrm{M}=8720, \mathrm{~T}=140, \mathrm{~V}=281, \mathrm{Xaa}=1$

$20 \mathrm{D}=277, \mathrm{De} \mathrm{l}=1, \mathrm{E}=8957, \mathrm{G}=4$

$74 \mathrm{~A}=1444$, Del=1, I=44, $\mathrm{L}=3, \mathrm{M}=29, \mathrm{P}=3, \mathrm{~S}=11, \mathrm{~T}=7691, \mathrm{~V}=13$

$18 \mathrm{Del}=1, \mathrm{I}=200, \mathrm{~L}=3, \mathrm{M}=9009, \mathrm{~V}=26$

$24 \mathrm{~N}=80, \mathrm{D}=8985, \mathrm{De}=1, \mathrm{Q}=1, \mathrm{E}=99, \mathrm{G}=23, \mathrm{~S}=42, \mathrm{~V}=7, \mathrm{Xaa}=1$

, $\mathrm{C}=4, \mathrm{H}=1, \mathrm{I}=3, \mathrm{M}=1, \mathrm{P}=1, \mathrm{~S}=7725, \mathrm{~T}=12$

$1 \mathrm{D}=1, \mathrm{De} 1=1, \mathrm{E}=9235, \mathrm{G}=1, \mathrm{~K}=1$

$1 \mathrm{Del}=1, \mathrm{~L}=9232, \mathrm{M}=5, \mathrm{P}=1$

$\mathrm{R}=9232, \mathrm{Del}=1, \mathrm{G}=1, \mathrm{~L}=1, \mathrm{~K}=4$

$14 \mathrm{R}=9065, \mathrm{Q}=4, \mathrm{I}=1, \mathrm{~K}=167, \mathrm{Xa} a=2$

$14 \mathrm{R}=9065, \mathrm{Q}=4, \mathrm{I}=1, \mathrm{~K}=167, \mathrm{Xaa}=2$
$1 \mathrm{H}=4, \mathrm{~F}=1, \mathrm{Y}=9232, \mathrm{Xaa}=2$

$1 \mathrm{R}=1, \mathrm{C}=1, \mathrm{G}=1, \mathrm{~W}=9235, \mathrm{Xaa}=1$

$\mathrm{O} A=9237, \mathrm{~S}=1, \mathrm{~V}=$

O $\mathrm{I}=9238, \mathrm{~K}=1$

6. $\mathrm{R}=9173, \mathrm{~K}=65, \mathrm{~W}=1$
$1 \mathrm{~A}=1, \mathrm{~N}=1, \mathrm{I}=1, \mathrm{M}=1, \mathrm{P}=3, \mathrm{~S}=1, \mathrm{~T}=923$

$1 \mathrm{~A}=1, \mathrm{~N}=1, \mathrm{I}=1, \mathrm{M}=1, \mathrm{P}=3, \mathrm{~S}=1, \mathrm{~T}=923$
$3 \mathrm{R}=9212, \mathrm{Q}=1, \mathrm{G}=1, \mathrm{I}=2, \mathrm{~K}=22, \mathrm{P}=1$

O $R=1, G=1, S=9237$

$0 \mathrm{G}=9238$, Xaa $=1$

$0 \mathrm{R}=1, \mathrm{G}=9237, \mathrm{~K}=1$

N $\mathrm{N}=9142, \mathrm{D}=3$, Del=1, $\mathrm{H}=1, \mathrm{~K}=1, \mathrm{~S}=16, \mathrm{~T}=75$

$6 \mathrm{~A}=10, \mathrm{~N}=40, \mathrm{Del}=1, \mathrm{P}=1, \mathrm{~S}=1, \mathrm{~T}=9186$

R $=9, \mathrm{~N}=1, \mathrm{Q}=9193, \mathrm{H}=25, \mathrm{~L}=1, \mathrm{~K}=8, \mathrm{Xaa}=2$

$8 \mathrm{R}=1, \mathrm{~N}=10, \mathrm{Q}=9161, \mathrm{H}=67$

I7 $\mathrm{R}=9019, \mathrm{I}=1, \mathrm{~K}=216, \mathrm{~S}=1, \mathrm{~W}=1, \mathrm{X} a \mathrm{a}=1$

$2 \mathrm{~A}=9221, \mathrm{E}=1, \mathrm{G}=3, \mathrm{~S}=6, \mathrm{~T}=5, \mathrm{~V}=3$
$\mathrm{~B} \mathrm{~A}=1, \mathrm{~F}=6, \mathrm{P}=1, \mathrm{~S}=9220, \mathrm{~T}=2, \mathrm{Y}=3, \mathrm{Xaa}=6$

O $\mathrm{A}=9236, \mathrm{~T}=3$

$0 \mathrm{Q}=9238, \mathrm{H}=1$
$14 \mathrm{I}=9059, \mathrm{~L}=1, \mathrm{~T}=3, \mathrm{~V}=176$

O $\mathrm{R}=1, \mathrm{~S}=9237, \mathrm{Xaa}=1$

$=8753, \mathrm{Xaa}=1$

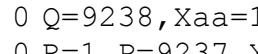

$2 \mathrm{~A}=8, \mathrm{P}=1, \mathrm{~S}=2, \mathrm{~T}=9227, \mathrm{xa} a=1$

$0 \mathrm{~L}=1, \mathrm{~F}=9237$, $\mathrm{Xaa}=1$

O $\mathrm{P}=1, \mathrm{~S}=9236, \mathrm{~T}=1, \mathrm{Xaa}=1$

$2 \mathrm{I}=13, \mathrm{~V}=9225, \mathrm{Xaa}=1$

1) $\mathrm{R}=9233, \mathrm{H}=1, \mathrm{Xaa}=1$

$22 \mathrm{~N}=8937, \mathrm{D}=8, \mathrm{G}=1, \mathrm{~K}=1, \mathrm{~S}=284, \mathrm{~T}=7, \mathrm{Xaa}=1$

1 I $=2, \mathrm{~L}=9232, \mathrm{~F}=2, \mathrm{P}=1, \mathrm{Xaa}=2$

$0 \mathrm{P}=9237, \mathrm{~S}=1, \mathrm{Xaa}=1$

$1 \mathrm{~L}=2, \mathrm{~F}=9235, \mathrm{~S}=1, \mathrm{Xaa}=1$

1) $\mathrm{R}=9232, \mathrm{C}=2, \mathrm{~K}=4, \mathrm{Xaa}=2$

$11 \mathrm{~A}=9127, \mathrm{~S}=63, \mathrm{~T}=35, \mathrm{~V}=13, \mathrm{Xaa}=1$

$2 \mathrm{~A}=4, \mathrm{I}=2, \mathrm{~S}=1, \mathrm{~T}=9228, \mathrm{Xaa}=4$

$8 \mathrm{I}=9151, \mathrm{M}=2, \mathrm{~V}=85, \mathrm{Xaa}=1$
$1 \mathrm{I}=2, \mathrm{~L}=3, \mathrm{~K}=1, \mathrm{M}=9231, \mathrm{~T}=1, \mathrm{xaa}=1$

$1 \mathrm{I}=2, \mathrm{~L}=3, \mathrm{~K}=1, \mathrm{M}=9231, \mathrm{~T}=1, \mathrm{Xa}=1$
$1 \mathrm{~A}=9235, \mathrm{~S}=1, \mathrm{~T}=1, \mathrm{~V}=1, \mathrm{xaa}=1$

$1 \mathrm{~A}=9235, \mathrm{~S}=1, \mathrm{~T}=1, \mathrm{~V}=1, \mathrm{xaa}=1$
$2 \mathrm{~A}=9221, \mathrm{~S}=9, \mathrm{~T}=3, \mathrm{~V}=5, \mathrm{xaa}=1$

$1 \mathrm{~L}=5, \mathrm{~F}=9232, \mathrm{Y}=1, \mathrm{Xaa}=1$

$24 \mathrm{~A}=115, \mathrm{~N}=4, \mathrm{I}=5, \mathrm{~K}=115, \mathrm{~S}=32, \mathrm{~T}=8966, \mathrm{~V}=1$, $\mathrm{Xaa}=1$ 


\section{vmuc:}

Not a mark

$443 \quad \mathrm{E}$

444 I

445 I

446 R

$448 \mathrm{M}$

$(>0.05)$

449

\begin{tabular}{ll|l}
\hline & N
\end{tabular}

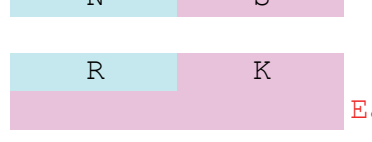

1990

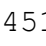

$\begin{array}{ll}451 & \text { A } \\ 452 & R\end{array}$

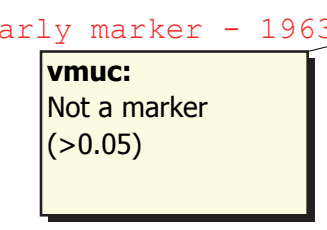

0,0041

454 E

455 D

$456 \mathrm{~V}$

457 S

$458 \quad \mathrm{~F}$

459 Q

460

$461 \mathrm{R}$

$462 \mathrm{G}$

$463 \mathrm{~V}$

$464 \mathrm{~F}$

$465 \mathrm{E}$

466 I

467 S

468 D

$469 \mathrm{E}$

$470 \mathrm{~K}$

471 A

472 T

$473 \mathrm{~N}$

$474 \quad \mathrm{P}$

475 I

$476 \mathrm{~V}$

$477 \quad \mathrm{P}$

478 S

479 F

480 D

$481 \mathrm{M}$

$482 \mathrm{~S}$

$483 \mathrm{~N}$

$484 \quad \mathrm{E}$

$485 \quad G$

$486 \mathrm{~S}$

$487 \mathrm{Y}$

488 F

$489 \mathrm{~F}$

$490 \quad G$

491 D

$492 \mathrm{~N}$

493 A

$495 \mathrm{E}$
$31 \mathrm{~A}=186, \mathrm{~N}=112, \mathrm{I}=11, \mathrm{M}=5, \mathrm{P}=30, \mathrm{~S}=23, \mathrm{~T}=8871, \mathrm{Xaa}=1$

$\mathrm{D}=6, \mathrm{E}=9232, \mathrm{Xaa}=1$

$\mathrm{A}=1, \mathrm{C}=1, \mathrm{G}=9234, \mathrm{~S}=2, \mathrm{Xaa}=1$

$0 \mathrm{R}=9237, \mathrm{D}=1, \mathrm{Xaa}=1$

$2 \mathrm{~A}=1, \mathrm{I}=13, \mathrm{~F}=1, \mathrm{~T}=9223$, Xaa $=1$

$1 \mathrm{R}=1, \mathrm{~F}=5, \mathrm{~S}=9229, \mathrm{~T}=1, \mathrm{Y}=1, \mathrm{Xaa}=2$

$1 \mathrm{D}=9235, \mathrm{E}=1, \mathrm{H}=1, \mathrm{Xaa}=2$

$1 \mathrm{E}=1, \mathrm{~L}=6, \mathrm{M}=9230, \mathrm{~V}=1, \mathrm{Xaa}=1$

1. $\mathrm{R}=9234, \mathrm{G}=2, \mathrm{~K}=1, \mathrm{~T}=1, \mathrm{Xaa}=1$

$5 \mathrm{~A}=9, \mathrm{E}=1, \mathrm{~S}=30, \mathrm{~T}=9198, \mathrm{Xaa}=1$

$1 \mathrm{E}=9235, \mathrm{G}=1, \mathrm{I}=1, \mathrm{~V}=1, \mathrm{Xaa}=1$

$5 \mathrm{I}=9189, \mathrm{~F}=1, \mathrm{~T}=3, \mathrm{~V}=45, \mathrm{Xaa}=1$

1 $\mathrm{I}=9230, \mathrm{~V}=8, \mathrm{xaa}=1$

$28 \mathrm{R}=8811, \mathrm{G}=3, \mathrm{I}=1, \mathrm{~K}=419, \mathrm{M}=2, \mathrm{~S}=1, \mathrm{Xaa}=2$

$7 \mathrm{I}=9, \mathrm{~L}=5, \mathrm{M}=9179, \mathrm{~T}=41, \mathrm{~V}=4, \mathrm{Xaa}=1$

$1 \mathrm{R}=1, \mathrm{I}=1, \mathrm{~L}=1, \mathrm{M}=9235, \mathrm{Xaa}=1$

$0 \mathrm{D}=1, \mathrm{E}=9238$

$01 \mathrm{R}=1, \mathrm{~N}=5571, \mathrm{D}=3, \mathrm{G}=13, \mathrm{H}=1, \mathrm{I}=14, \mathrm{~K}=1, \mathrm{~S}=3633, \mathrm{~T}=2$

$8 \mathrm{~A}=9164, \mathrm{C}=1, \mathrm{~S}=40, \mathrm{~T}=22, \mathrm{~V}=12$

$42 \mathrm{R}=8468, \mathrm{Q}=3, \mathrm{G}=4, \mathrm{~K}=761, \mathrm{~S}=2, \mathrm{~T}=1$

$7 \mathrm{~A}=4, \mathrm{Q}=1, \mathrm{~L}=6, \mathrm{P}=9179, \mathrm{~S}=38, \mathrm{~T}=9, \mathrm{Xaa}=2$

$8 \mathrm{D}=81, \mathrm{E}=9150, \mathrm{G}=7, \mathrm{~K}=1$

$3 \mathrm{~N}=7, \mathrm{D}=9215, \mathrm{E}=15, \mathrm{G}=1, \mathrm{~V}=1$

$9 \mathrm{~A}=3, \mathrm{~L}=57, \mathrm{M}=26, \mathrm{~V}=9153$

$0 \mathrm{~F}=1, \mathrm{~S}=9238$

$\mathrm{F}=9236, \mathrm{~S}=2, \mathrm{Xaa}=1$

$2 \mathrm{R}=3, \mathrm{Q}=9226, \mathrm{H}=8, \mathrm{~K}=1, \mathrm{Xaa}=1$

$1 \mathrm{R}=5, \mathrm{E}=1, \mathrm{G}=9233$

$\mathrm{R}=9237, \mathrm{P}=1, \mathrm{~W}=1$

$0 \mathrm{~A}=1, \mathrm{G}=9238$

$0 \mathrm{~A}=3, \mathrm{~V}=9236$

$\mathrm{R}=1, \mathrm{~F}=9237, \mathrm{~S}=1$

$1 \mathrm{~A}=1, \mathrm{E}=9235, \mathrm{G}=2, \mathrm{~K}=1$

$5 \mathrm{I}=27, \mathrm{~L}=9191, \mathrm{~F}=20, \mathrm{~V}=1$

$1 \mathrm{~A}=2, \mathrm{P}=1, \mathrm{~S}=9231, \mathrm{~T}=3, \mathrm{~W}=1, \mathrm{Y}=1$

$1 \mathrm{~A}=1, \mathrm{~N}=1, \mathrm{D}=9234, \mathrm{E}=2$

$1 \mathrm{D}=2, \mathrm{E}=9234, \mathrm{~K}=1, \mathrm{~S}=1, \mathrm{Xaa}=1$

$1 \mathrm{R}=2, \mathrm{~N}=1, \mathrm{E}=1, \mathrm{~K}=9233, \mathrm{~T}=2$

$\mathrm{A}=9237, \mathrm{R}=1, \mathrm{I}=1$

$4 \mathrm{~A}=33, \mathrm{R}=1, \mathrm{~K}=1, \mathrm{M}=1, \mathrm{~S}=1, \mathrm{~T}=9202$

$4 \mathrm{~N}=9202, \mathrm{H}=2, \mathrm{~K}=1, \mathrm{~S}=33, \mathrm{~T}=1$

$1 \mathrm{P}=9229, \mathrm{~S}=9, \mathrm{~T}=1$

$2 \mathrm{I}=9223, \mathrm{~T}=2, \mathrm{~V}=1$

$\mathrm{A}=3, \mathrm{~L}=1, \mathrm{~V}=9235$

$\mathrm{O} \mathrm{P}=9239$

$2 \mathrm{~F}=9, \mathrm{P}=3, \mathrm{~S}=9227$

$2 \mathrm{~L}=14, \mathrm{~F}=9224, \mathrm{~V}=1$

$3 \mathrm{~N}=10, \mathrm{D}=9214, \mathrm{E}=14, \mathrm{G}=1$

$\mathrm{H}=1, \mathrm{I}=15, \mathrm{~L}=3, \mathrm{~K}=2, \mathrm{M}=9182, \mathrm{~T}=1, \mathrm{~V}=35$

$71 \mathrm{~N}=1713, \mathrm{D}=1, \mathrm{C}=1, \mathrm{Del}=2, \mathrm{E}=1, \mathrm{G}=2, \mathrm{I}=1, \mathrm{~S}=7511, \mathrm{~T}=7$

$27 \mathrm{~N}=8829, \mathrm{D}=4, \mathrm{~K}=404, \mathrm{~S}=1, \mathrm{Y}=1$

$3 \mathrm{D}=27, \mathrm{E}=9209, \mathrm{G}=2, \mathrm{~V}=1$

$1 \mathrm{R}=4, \mathrm{E}=1, \mathrm{G}=9233, \mathrm{~S}=1$

$1 \mathrm{I}=1, \mathrm{~F}=1, \mathrm{P}=1, \mathrm{~S}=9232, \mathrm{~T}=2, \mathrm{Y}=1, \mathrm{Xaa}=1$

$\mathrm{N}=1, \mathrm{H}=2, \mathrm{Y}=9236$

$1 \mathrm{~F}=9232, \mathrm{~S}=7$

$1 \mathrm{~L}=1, \mathrm{~F}=9234, \mathrm{~S}=4$

$\mathrm{O} \mathrm{E}=2, \mathrm{G}=9236, \mathrm{~V}=1$

$1 \mathrm{~A}=3, \mathrm{~N}=2, \mathrm{D}=9230, \mathrm{E}=3, \mathrm{G}=1$

$5 \mathrm{~N}=9203, \mathrm{D}=4, \mathrm{H}=7, \mathrm{I}=2, \mathrm{~K}=3, \mathrm{~S}=16, \mathrm{~T}=3, \mathrm{Y}=1$

$\mathrm{A} A=9239$

$4 \mathrm{D}=21, \mathrm{E}=9201, \mathrm{G}=2, \mathrm{~K}=14, \mathrm{Xaa}=1$

$3 \mathrm{D}=20, \mathrm{E}=9213, \mathrm{G}=1, \mathrm{~K}=5$

$12 \mathrm{H}=1, \mathrm{I}=3, \mathrm{~F}=135, \mathrm{~S}=1, \mathrm{Y}=9098$

$17 \mathrm{~A}=1, \mathrm{~N}=1, \mathrm{D}=9026, \mathrm{E}=202, \mathrm{G}=2, \mathrm{H}=1, \mathrm{~T}=2, \mathrm{Y}=3$

$14 \mathrm{~N}=9073, \mathrm{D}=1, \mathrm{H}=5, \mathrm{I}=5, \mathrm{~K}=5, \mathrm{~S}=145, \mathrm{Y}=1, \mathrm{Xaa}=3$ 


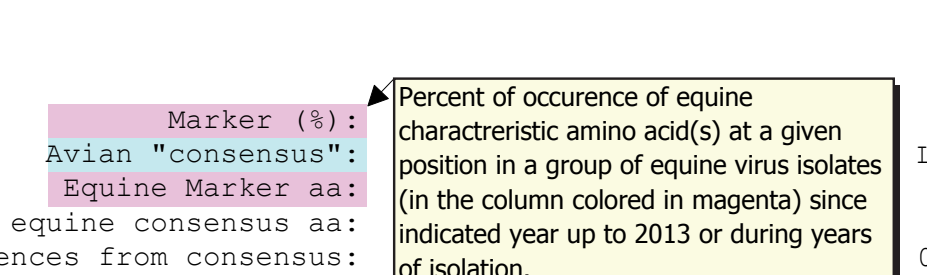

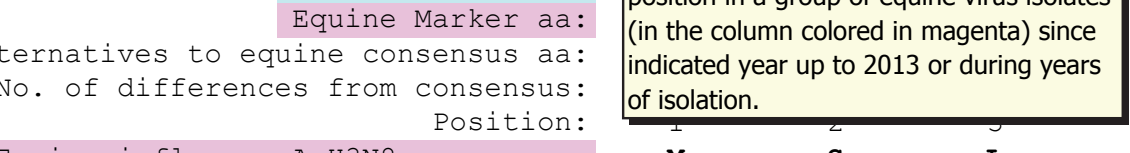

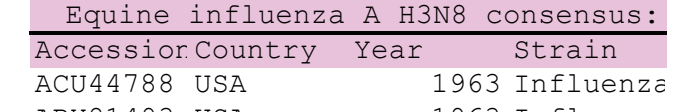

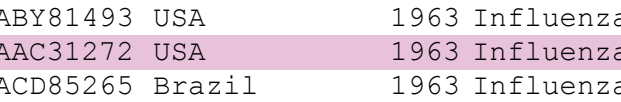

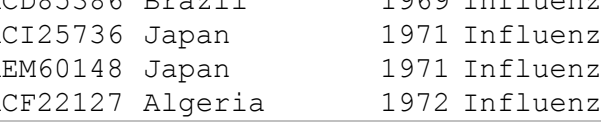

\begin{tabular}{|c|c|c|}
\hline 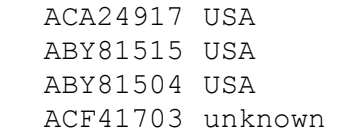 & 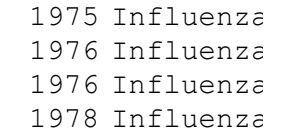 & \\
\hline & 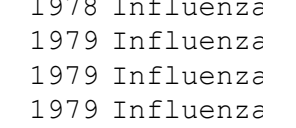 & Legend: \\
\hline & 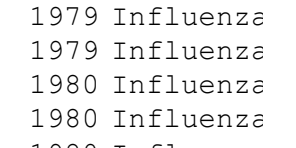 & 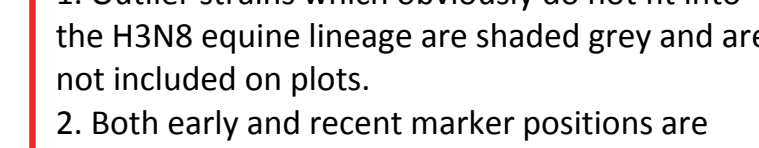 \\
\hline & 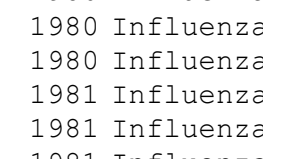 & 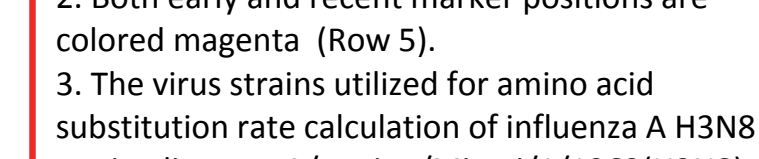 \\
\hline 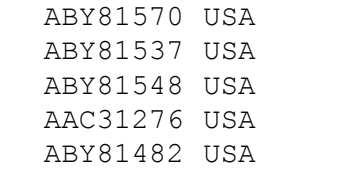 & 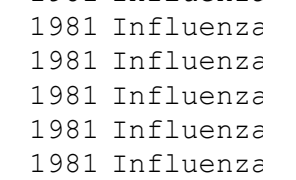 & 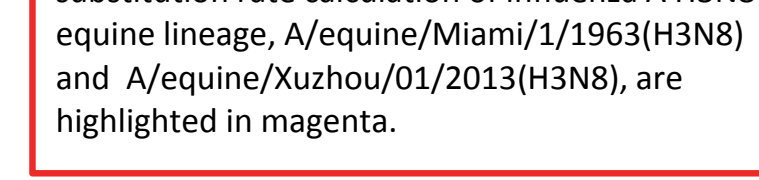 \\
\hline
\end{tabular}

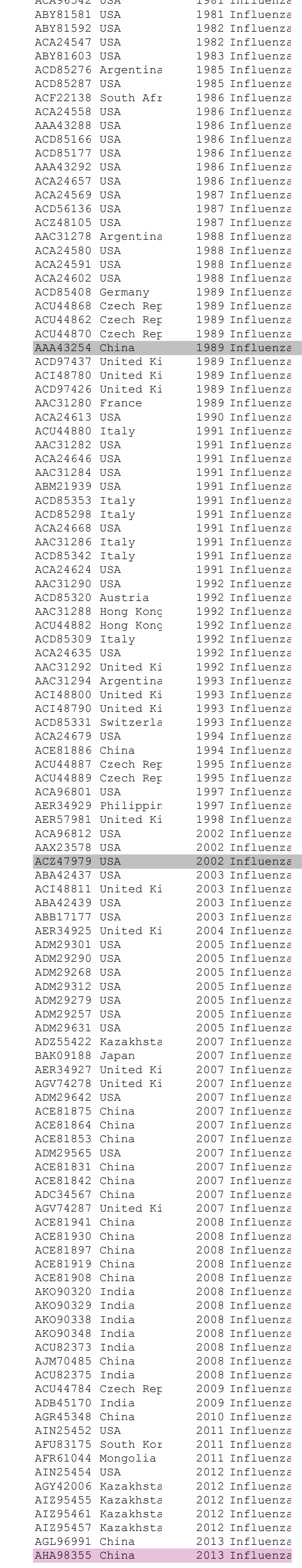

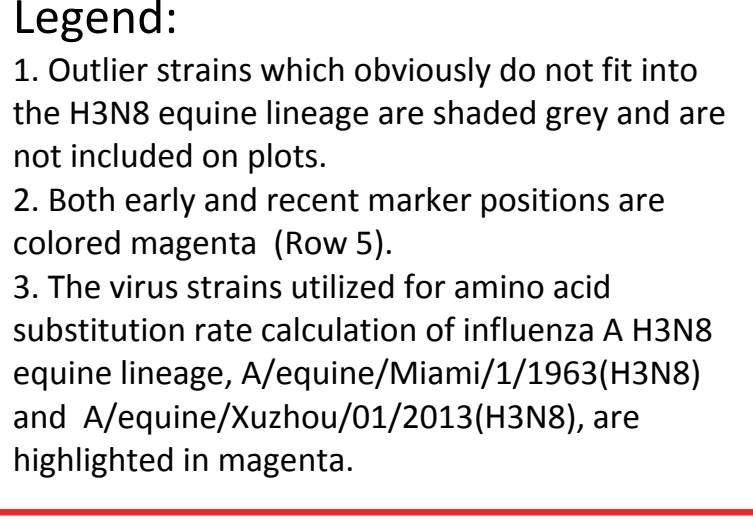


(5)

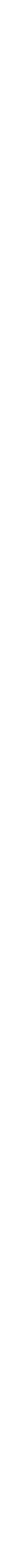




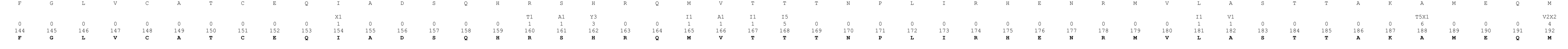


<smiles>CC(C)(C)C</smiles> 


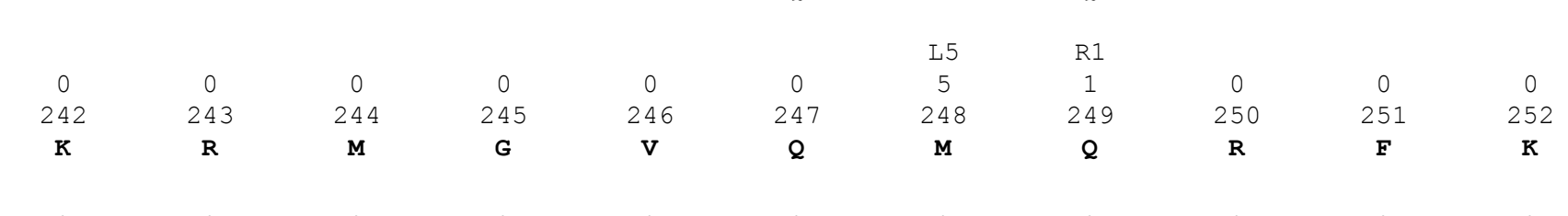

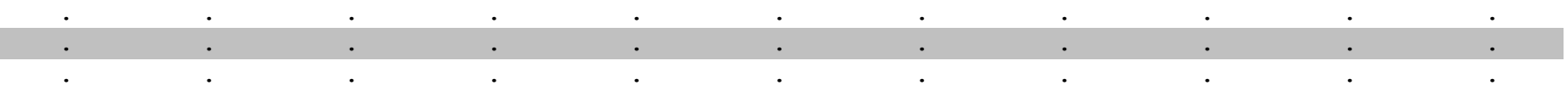




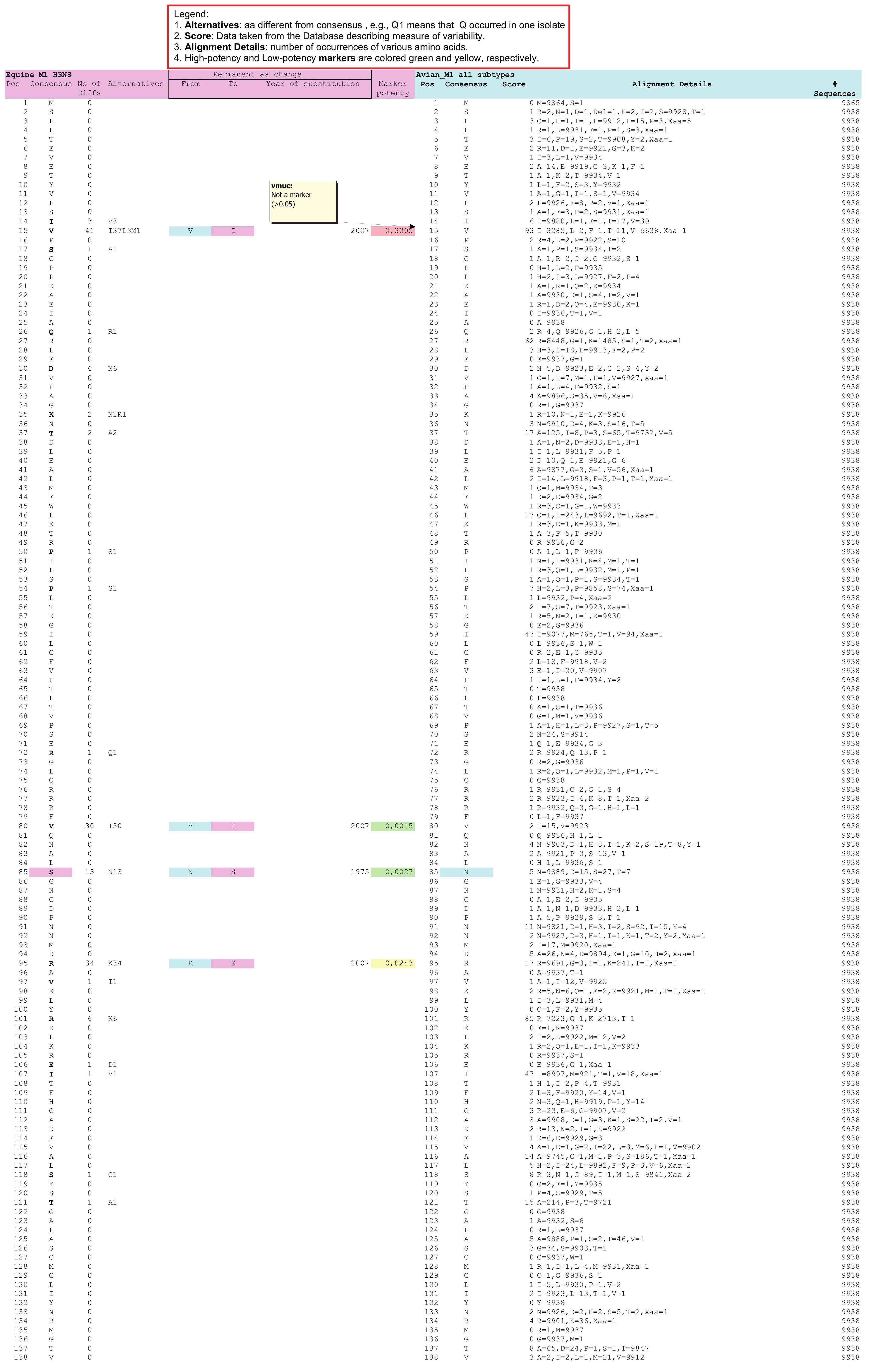


$49 \mathrm{~L}=1054, \mathrm{~F}=8883$, Xaa $=1$

O $\mathrm{D}=1, \mathrm{G}=9936, \mathrm{Xaa}=$

$2 \mathrm{~A}=1, \mathrm{I}=2, \mathrm{~L}=8, \mathrm{M}=2, \mathrm{~V}=9924, \mathrm{Xaa}=1$

$\mathrm{O} C=9937, \mathrm{Xaa}=$

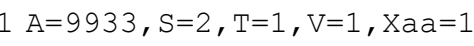

$2 \mathrm{~A}=2, \mathrm{I}=8, \mathrm{M}=2, \mathrm{~S}=1, \mathrm{~T}=9924$, $\mathrm{Xaa}=1$

$0 \mathrm{C}=9935, \mathrm{~S}=1, \mathrm{Xaa}=2$

$1 \mathrm{R}=1, \mathrm{Q}=9933, \mathrm{H}=2, \mathrm{P}=1, \mathrm{Xaa}=1$

$1 \mathrm{R}=1, \mathrm{Q}=9933, \mathrm{H}=2, \mathrm{P}=1, \mathrm{X} a$
$\mathrm{O}=1, \mathrm{I}=9935, \mathrm{~T}=1, \mathrm{Xaa}=1$

$1 \mathrm{~A}=9933, \mathrm{G}=1, \mathrm{~S}=1, \mathrm{~V}=2, \mathrm{Xaa}=1$

$1 \mathrm{~N}=1, \mathrm{D}=9931, \mathrm{E}=4, \mathrm{H}=1, \mathrm{Xaa}=1$

$31 \mathrm{~A}=547, \mathrm{G}=2, \mathrm{~L}=1, \mathrm{~S}=9386, \mathrm{~T}=1, \mathrm{Xaa}=1$

$23 \mathrm{R}=1, \mathrm{Q}=9581, \mathrm{H}=354, \mathrm{Xaa}=2$

$\mathrm{O} \mathrm{Q}=1, \mathrm{H}=9936, \mathrm{Xaa}=1$

$1 \mathrm{R}=9934, \mathrm{Q}=2, \mathrm{~K}=1, \mathrm{Xaa}=1$

$4 \mathrm{~A}=31, \mathrm{~F}=2, \mathrm{P}=3, \mathrm{~S}=9901, \mathrm{Xaa}=1$

$1 \mathrm{Q}=5, \mathrm{H}=9932, \mathrm{Xaa}=1$

$1 \mathrm{R}=9929, \mathrm{G}=8, \mathrm{Xaa}=1$

$1 \mathrm{R}=1, \mathrm{Del}=1, \mathrm{Q}=9930, \mathrm{~L}=3, \mathrm{~K}=2, \mathrm{Xa} a=1$

$2 \mathrm{I}=8, \mathrm{I}=2, \mathrm{M}=9926, \mathrm{~V}=1, \mathrm{Xa} a=1$

$88 \mathrm{~A}=2590, \mathrm{I}=37, \mathrm{M}=8, \mathrm{~T}=10, \mathrm{~V}=7292, \mathrm{Xaa}=1$

$32 \mathrm{~A}=493, \mathrm{~N}=5, \mathrm{I}=12, \mathrm{~S}=8, \mathrm{~T}=9417, \mathrm{~V}=1, \mathrm{Xaa}=2$

$65 \mathrm{~A}=18, \mathrm{I}=1568, \mathrm{~K}=1, \mathrm{P}=1, \mathrm{~T}=8349, \mathrm{Xaa}=1$

$2 \mathrm{~N}=1, \mathrm{I}=13, \mathrm{~S}=2, \mathrm{~T}=9921, \mathrm{Xaa}=1$

$1 \mathrm{~N}=9929, \mathrm{D}=1, \mathrm{~S}=5, \mathrm{~T}=2$,

O $\mathrm{P}=9936, \mathrm{~T}=1, \mathrm{Xaa}=1$

$1 \mathrm{I}=2, \mathrm{~L}=9932, \mathrm{M}=3, \mathrm{Xaa}=$

$5 \mathrm{R}=9888, \mathrm{G}=1, \mathrm{~K}=47, \mathrm{~S}=1, \mathrm{Xaa}=1$

$0 \mathrm{R}=1, \mathrm{H}=99936, \mathrm{Xa} a=1$

$\mathrm{R}=1, \mathrm{E}=9935, \mathrm{G}=1, \mathrm{Xa} a=$

$1 \mathrm{~N}=9929, \mathrm{D}=2, \mathrm{~K}=1, \mathrm{~S}=4, \mathrm{~T}=1, \mathrm{Xaa}=1$

O $\mathrm{R}=9936, \mathrm{~K}=1, \mathrm{Xaa}=1$

O $I=1, M=9935, V=1, X a a=1$

$0 \mathrm{G}=1, \mathrm{~V}=9936, \mathrm{Xaa}=1$

$6 \mathrm{I}=20, \mathrm{~L}=9886, \mathrm{M}=26$

$1 \mathrm{~A}=9933, \mathrm{~T}=4, \mathrm{Xaa}=1$

$1 \mathrm{C}=1, \mathrm{G}=1, \mathrm{I}=1, \mathrm{~S}=9934$, Xaa $=1$

$1 \mathrm{~A}=1, \mathrm{H}=1, \mathrm{I}=4, \mathrm{P}=2, \mathrm{~T}=9929, \mathrm{x}$

$1 \mathrm{~A}=1, \mathrm{P}=2, \mathrm{~T}=9934, \mathrm{Xaa}=1 \mathrm{X}, \mathrm{Xaa}=1$

$1 \mathrm{R}=4, \mathrm{~K}=9933, \mathrm{Xaa}=1$

$0 \mathrm{~A}=9937, \mathrm{Xaa}=1$

$1 \mathrm{I}=1, \mathrm{M}=9933, \mathrm{~V}=3, \mathrm{Xaa}=1$

$0 \mathrm{E}=9936, \mathrm{G}=1, \mathrm{Xaa}=1$

$2 \mathrm{R}=3, \mathrm{Q}=9923, \mathrm{H}=4, \mathrm{~L}=2, \mathrm{~K}=1, \mathrm{Xaa}=5$

$21 \mathrm{I}=45, \mathrm{M}=9641, \mathrm{~V}=250, \mathrm{Xaa}=2$

$1 \mathrm{~A}=9933, \mathrm{D}=1, \mathrm{E}=1, \mathrm{P}=1, \mathrm{~V}=1, \mathrm{Xaa}=1$

$2 \mathrm{~A}=10, \mathrm{~L}=1, \mathrm{P}=1, \mathrm{~S}=9920, \mathrm{~T}=4, \mathrm{~W}=1, \mathrm{Xaa}=1$

$2 \mathrm{~N}=18, \mathrm{I}=2, \mathrm{~K}=1, \mathrm{~S}=9916, \mathrm{Xaa}=1$

$1 \mathrm{D}=1, \mathrm{Q}=2, \mathrm{E}=9932, \mathrm{G}=1, \mathrm{~K}=1, \mathrm{Xaa}=1$

$2 \mathrm{R}=2, \mathrm{Q}=9919, \mathrm{H}=15, \mathrm{P}=1, \mathrm{Xaa}=1$

$5 \mathrm{~A}=9890, \mathrm{E}=1, \mathrm{M}=13, \mathrm{P}=1, \mathrm{~S}=12, \mathrm{~T}=3, \mathrm{~V}=17, \mathrm{Xaa}=1$

$2 \mathrm{D}=6, \mathrm{E}=9925, \mathrm{~K}=6, \mathrm{Xaa}=$

$1 \mathrm{~A}=9931, \mathrm{R}=3, \mathrm{P}=1, \mathrm{~T}=1, \mathrm{~V}=1, \mathrm{Xaa}=1$

2 $\mathrm{I}=8, \mathrm{M}=9924, \mathrm{~V}=5, \mathrm{Xaa}=1$

$3 \mathrm{D}=22, \mathrm{~B}=9913, \mathrm{~K}=2, \mathrm{Xaa}=1$

$29 \mathrm{~A}=6, \mathrm{I}=452, \mathrm{~T}=7, \mathrm{~V}=9471, \mathrm{Xaa}=$

$1 \mathrm{~A}=9932, \mathrm{G}=1, \mathrm{P}=1, \mathrm{~V}=3, \mathrm{Xa} a=1$

$77 \mathrm{~N}=1698, \mathrm{C}=7, \mathrm{G}=127, \mathrm{I}=1, \mathrm{~K}=1, \mathrm{~S}=8102, \mathrm{~T}=1, \mathrm{Xaa}=1$

$1 \mathrm{R}=3, \mathrm{Q}=9931, \mathrm{H}=1, \mathrm{P}=1, \mathrm{~S}=1, \mathrm{Xaa}=1$

$6 \mathrm{~A}=9875, \mathrm{R}=1, \mathrm{G}=2, \mathrm{~T}=60$

$1 \mathrm{R}=9931, \mathrm{~K}=7$

$4 \mathrm{R}=3, \mathrm{Q}=9903, \mathrm{E}=1, \mathrm{H}=19, \mathrm{~L}=1, \mathrm{~K}=10, \mathrm{Xaa}=1$

O $\mathrm{I}=2, \mathrm{M}=9935, \mathrm{~V}=1$

$1 \mathrm{~A}=1, \mathrm{E}=2, \mathrm{G}=5, \mathrm{I}=2, \mathrm{~V}=9928$

$4 \mathrm{R}=2, \mathrm{Q}=9893, \mathrm{H}=42, \mathrm{Xaa}=1$

$1 \mathrm{~A}=9933, \mathrm{~S}=1, \mathrm{~T}=4$

$0 \mathrm{M}=9937, \mathrm{~V}=1$

$\mathrm{R}=9936, \mathrm{I}=1, \mathrm{~K}=1$

$20 \mathrm{~A}=289, \mathrm{~N}=1, \mathrm{I}=1, \mathrm{P}=1, \mathrm{~S}=4, \mathrm{~T}=9642$

$25 \mathrm{I}=9540, \mathrm{M}=2, \mathrm{~T}=1, \mathrm{~V}=395$

$0 \mathrm{R}=1, \mathrm{G}=9936, \mathrm{~V}=1$

$26 \mathrm{R}=4, \mathrm{~N}=17, \mathrm{Q}=373, \mathrm{H}=9540, \mathrm{~L}=2, \mathrm{~S}=1, \mathrm{Xaa}=1$

$2 \mathrm{H}=22, \mathrm{P}=9916$

$70 \mathrm{R}=6, \mathrm{~N}=1806, \mathrm{G}=2, \mathrm{~K}=3, \mathrm{~S}=8118, \mathrm{~T}=1, \mathrm{Y}=1, \mathrm{Xaa}=1$

$25 \mathrm{C}=2, \mathrm{I}=1, \mathrm{~F}=1, \mathrm{~S}=9529, \mathrm{~T}=405$

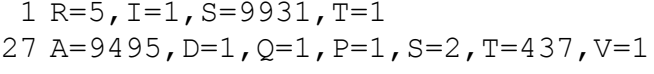

$2 R=15, D=1, G=9919, I=1, S=2$

$2 \mathrm{Q}=1, \mathrm{~L}=9923, \mathrm{M}=8, \mathrm{~V}=2, \mathrm{Xaa}=4$

$68 \mathrm{R}=1811, \mathrm{~K}=8127$

$6 \mathrm{~N}=28, \mathrm{D}=9877, \mathrm{E}=31, \mathrm{H}=1, \mathrm{~V}=1$

$67 \mathrm{~N}=1742, \mathrm{D}=8196$

$1 \mathrm{I}=1, \mathrm{~L}=9933, \mathrm{~F}=3, \mathrm{~V}=1$

$44 \mathrm{I}=901, \mathrm{~L}=903$

$3 \mathrm{D}=7, \mathrm{E}=9911, \mathrm{G}=12, \mathrm{~K}=8$

$2 \mathrm{~N}=9924, \mathrm{D}=1, \mathrm{H}=1, \mathrm{I}=1, \mathrm{~K}=4, \mathrm{~S}=1, \mathrm{~T}=5, \mathrm{Y}=1$

$1 \mathrm{~L}=9933, \mathrm{~S}=2, \mathrm{~W}=2, \mathrm{~V}=1$

$\mathrm{O} \mathrm{Q}=9936, \mathrm{~L}=1, \mathrm{Xaa}=1$

$4 \mathrm{~A}=9905, \mathrm{G}=4, \mathrm{P}=1, \mathrm{~T}=24, \mathrm{~V}=4$

$2 \mathrm{H}=2, \mathrm{~F}=11, \mathrm{Y}=9925$

$1 \mathrm{Q}=9930, \mathrm{H}=5, \mathrm{P}=1, \mathrm{Xaa}=2$

$7 \mathrm{R}=13, \mathrm{~N}=45, \mathrm{~K}=9869, \mathrm{~S}=1, \mathrm{~T}=10$

$0 \mathrm{R}=9935, \mathrm{P}=2$, $\mathrm{W}=1$

$1 \mathrm{R}=1, \mathrm{~L}=1, \mathrm{~K}=2, \mathrm{M}=9934$

$0 \mathrm{E}=1, \mathrm{G}=9937$

$12 \mathrm{~A}=1, \mathrm{G}=6, \mathrm{~L}=124, \mathrm{M}=16, \mathrm{~V}=9791$

$\mathrm{O} \mathrm{Q}=9937, \mathrm{H}=$

$29 \mathrm{I}=53, \mathrm{~L}=362, \mathrm{M}=9510, \mathrm{~V}=12$, $\mathrm{Xaa}=1$

$4 \mathrm{R}=5, \mathrm{Q}=9904, \mathrm{H}=28, \mathrm{~L}=1$

$3 \mathrm{~L}=25, \mathrm{~F}=9913$ 


Alternatives: aa different from consensus, e.g., Q1 means that $Q$ occurred in one isolate

2. Score: Data taken from the Database describing measure of variability

3. Alignment Details: number of occurrences of various amino acids.

4. High-potency and Low-potency markers are colored green and yellow, respectively.

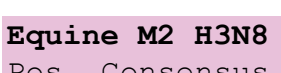

Equine M2 H3N8
Pos Consensus No of Alternatives

\begin{tabular}{ccc}
\hline \multicolumn{3}{c|}{ Permanent aa change } \\
\hline From & To Year of substitution
\end{tabular}

Marker
potency

Avian_M2 all subtypes
Pos Consensus Score

S 1

K1

E4

Y1
N5R1

N2D1

D20V1

L1

I1

A2

V5T1

F1

S1
S26

C13

A1

H2

H1L

R1

M14

Q1 $26 \mathrm{~K} 1$

G1

V1

D1

F1

S 9

T11D1

S29N1

E2

S 11

Y1

G2V1
$2007 \quad 0,0017$

$1975 \quad 0,0057$

vmuc:

not a marker $\begin{array}{llll}1 & M & 0 & M=9555 \\ 2 & \mathrm{~S} & 0 & \mathrm{I}=1, \mathrm{~S}=9569, \mathrm{~T}=1\end{array}$

Alignment Details

$\mathrm{C}=1, \mathrm{H}=1, \mathrm{~L}=9552, \mathrm{~F}=12, \mathrm{P}=1, \mathrm{~V}=1, \mathrm{Xaa}=3$

$\mathrm{R}=1, \mathrm{~L}=9566, \mathrm{P}=1, \mathrm{~S}=2, \mathrm{Xaa}=1$

$\mathrm{N}=1, \mathrm{P}=7, \mathrm{~S}=2, \mathrm{~T}=9559, \mathrm{Y}=2$

$\mathrm{De}=1, \mathrm{E}=9567, \mathrm{G}=2, \mathrm{~K}=1$

$\mathrm{A}=19, \mathrm{Del}=1, \mathrm{E}=9547, \mathrm{G}=3, \mathrm{~K}=1$

$\mathrm{A}=1, \mathrm{R}=1, \mathrm{Del}=1, \mathrm{P}=1, \mathrm{~T}=9567$

$\mathrm{Del}=38, \mathrm{H}=319, \mathrm{~L}=477, \mathrm{P}=8737, \mathrm{~S}=$

$\mathrm{Del}=38, \mathrm{I}=587, \mathrm{~S}=11, \mathrm{~T}=8935$, Xaa $=1$

$\mathrm{R}=9314, \mathrm{De} 1=38, \mathrm{~K}=217, \mathrm{P}=1, \mathrm{~T}=1, \mathrm{Xaa}=1$

$A=2, N=8770, D=8, D e 1=37, H=6, I=1, K=136, S=526, T=85, X a a=1$

$\mathrm{A}=6, \mathrm{R}=1, \mathrm{D}=1, \mathrm{Del}=37, \mathrm{E}=1536, \mathrm{G}=7989, \mathrm{~V}=2$

$\mathrm{R}=2, \mathrm{G}=1, \mathrm{~W}=9569$

$\mathrm{D}=8, \mathrm{E}=9206, \mathrm{G}=337, \mathrm{~K}=1, \mathrm{~V}=2$

$\mathrm{R}=1, \mathrm{C}=9532, \mathrm{G}=1, \mathrm{~F}=1, \mathrm{Y}=36, \mathrm{X} \mathrm{a}=1$

$\mathrm{R}=3732, \mathrm{~N}=333, \mathrm{I}=1, \mathrm{~K}=5496, \mathrm{~S}=9$, $\mathrm{Xaa}=1$

$R=17, N=224, C=1, G=3, I=27, K=17, S=9282, X a a=1$

$\mathrm{D}=9330, \mathrm{G}=231, \mathrm{~V}=11$

$\mathrm{L}=36, \mathrm{~S}=9536$

$\mathrm{N}=50, \mathrm{C}=2, \mathrm{G}=8, \mathrm{~S}=9510, \mathrm{Xaa}=1$

$\mathrm{N}=47, \mathrm{D}=9516, \mathrm{E}=8$

$\mathrm{H}=2, \mathrm{~L}=10, \mathrm{P}=9536, \mathrm{~S}=12, \mathrm{~T}=9$, $\mathrm{Xaa}=2$

$\mathrm{R}=1, \mathrm{I}=278, \mathrm{~L}=9270, \mathrm{~F}=20, \mathrm{~V}=2$

$\mathrm{A}=82, \mathrm{E}=1, \mathrm{G}=6, \mathrm{I}=773, \mathrm{~L}=4, \mathrm{M}=4, \mathrm{~F}=3, \mathrm{~T}=10, \mathrm{~V}=8686, \mathrm{Xaa}=2$

$\mathrm{A}=16, \mathrm{D}=9, \mathrm{I}=7378, \mathrm{~L}=34, \mathrm{M}=1, \mathrm{~F}=181, \mathrm{~S}=3, \mathrm{~T}=17, \mathrm{Y}=2, \mathrm{~V}=1930$

$\mathrm{A}=9464, \mathrm{D}=1, \mathrm{G}=1, \mathrm{~S}=16, \mathrm{~T}=48, \mathrm{~V}=41$

$\mathrm{A}=9548, \mathrm{E}=1, \mathrm{~S}=8, \mathrm{I}=12, \mathrm{xaa}=2$

$\mathrm{R}=2, \mathrm{~N}=646, \mathrm{D}=2, \mathrm{G}=5, \mathrm{I}=1, \mathrm{~K}=1, \mathrm{~S}=8913, \mathrm{Xaa}=1$

$\mathrm{I}=9283, \mathrm{~F}=4, \mathrm{~T}=6, \mathrm{~V}=277, \mathrm{Xaa}=1$

$I=9518,1$
$G=9571$

$I=9523, M=1, S=6, T=1, V=38, X a a=2$

$\mathrm{L}=9566, \mathrm{M}=1, \mathrm{~W}=1, \mathrm{~V}=3$

$\mathrm{D}=1, \mathrm{Q}=1, \mathrm{H}=9568, \mathrm{P}$

$\mathrm{R}=1, \mathrm{I}=9488, \mathrm{~K}=2, \mathrm{M}=11, \mathrm{~T}=28, \mathrm{~V}=41$

$\mathrm{L}=9564, \mathrm{M}=2, \mathrm{~F}=3, \mathrm{~S}=1, \mathrm{~W}=1$

$\mathrm{R}=1, \mathrm{C}=2, \mathrm{G}=1, \mathrm{~L}=1, \mathrm{~W}=9566$

$\mathrm{I}=9526, \mathrm{M}=9, \mathrm{~S}=1, \mathrm{~N}=24, \mathrm{~V}=10, \mathrm{Xaa}=1$

$\mathrm{A}=1, \mathrm{I}=15, \mathrm{~L}=9381, \mathrm{~F}=164, \mathrm{P}=1, \mathrm{~S}=3, \mathrm{~T}=6$

$\mathrm{N}=35, \mathrm{D}=9531, \mathrm{E}=1, \mathrm{Y}=3, \mathrm{~V}=1$

$\mathrm{R}=9541, \mathrm{C}=3, \mathrm{H}=25, \mathrm{~L}=1, \mathrm{~S}=$

$\mathrm{I}=8, \mathrm{~L}=9558, \mathrm{~F}=4, \mathrm{P}=1$

$\mathrm{L}=32, \mathrm{~F}=9538, \mathrm{Y}=1$

$\mathrm{L}=8, \mathrm{P}=9545, \mathrm{~S}=16, \mathrm{Y}=1, \mathrm{~V}=$

$\mathrm{R}=4, \mathrm{Q}=2, \mathrm{~K}=9565$

$\mathrm{R}=1, \mathrm{C}=9343, \mathrm{~F}=55, \mathrm{~S}=1, \mathrm{~W}=3, \mathrm{Y}=168$

$A=1, I=9348, I=4, I=11, V=207$

$\mathrm{N}=1, \mathrm{C}=86, \mathrm{H}=52, \mathrm{I}=10, \mathrm{~S}=4, \mathrm{Y}=9418$

$\mathrm{R}=9531, \mathrm{C}=17, \mathrm{H}=13, \mathrm{I}=1, \mathrm{~L}=3, \mathrm{~S}=5, \mathrm{X} a \mathrm{a}=1$

$\mathrm{I}=116, \mathrm{~L}=8186, \mathrm{~F}=1265, \mathrm{Xa} a=4$

$\mathrm{Xaa}=$

$\mathrm{A}=6, \mathrm{R}=4, \mathrm{D}=32, \mathrm{E}=100, \mathrm{G}=9416, \mathrm{~S}=7, \mathrm{~V}=$

$\mathrm{I}=9566, \mathrm{D}=2, \mathrm{P}=2, \mathrm{~V}=1$

$\mathrm{R}=3, \mathrm{Q}=11, \mathrm{E}=1, \mathrm{~K}=9556$

$\mathrm{R}=9214, \mathrm{~N}=1, \mathrm{E}=1, \mathrm{G}=116, \mathrm{I}=18, \mathrm{~K}=187, \mathrm{~S}=34$

$R=7, E=1, G=9559, S=2, W=1, V=1$

$\mathrm{I}=1, \mathrm{P}=9567, \mathrm{~S}=1, \mathrm{~T}=1, \mathrm{Xa}=1$

$\mathrm{A}=286, \mathrm{~F}=32, \mathrm{P}=4, \mathrm{~S}=9234, \mathrm{~T}=5, \mathrm{Y}=10$

$\mathrm{A}=10, \mathrm{R}=7, \mathrm{~K}=28, \mathrm{M}=33, \mathrm{~S}=1, \mathrm{~T}=9491, \mathrm{~V}=1$

$\mathrm{A}=486, \mathrm{E}=9003, \mathrm{G}=24, \mathrm{~K}=32, \mathrm{~T}=24, \mathrm{~V}=1, \mathrm{Xaa}=1$

$\mathrm{R}=8, \mathrm{G}=9563$

$\mathrm{A}=22, \mathrm{I}=65, \mathrm{~L}=28, \mathrm{M}=99, \mathrm{~T}=2, \mathrm{~V}=9355$

$\mathrm{L}=4, \mathrm{P}=9567$

$\mathrm{R}=1, \mathrm{D}=1, \mathrm{Q}=1, \mathrm{E}=9374, \mathrm{G}=3, \mathrm{~K}=182, \mathrm{~T}=8, \mathrm{~V}=1$

$A=1, C=1, F=3, S=9564, Y=2$

$\mathrm{I}=2, \mathrm{M}=9568, \mathrm{~T}=1$

$\mathrm{R}=9561, \mathrm{~K}=6, \mathrm{M}=2, \mathrm{~T}=2$

$\mathrm{A}=6, \mathrm{D}=3, \mathrm{E}=9555, \mathrm{G}=2, \mathrm{~K}=4, \mathrm{Xa} a=1$

$\mathrm{D}=2, \mathrm{Q}=1, \mathrm{E}=9559, \mathrm{G}=2, \mathrm{~K}=7$

$\mathrm{N}=1, \mathrm{C}=2, \mathrm{~F}=3, \mathrm{~S}=1, \mathrm{Y}=9564$

$R=9433, Q=132, L=1, P=2, W=3$

$R=1, Q=9537, E=2, H=23, L=4, K=1, F=1, P=2$

$\mathrm{E}=9544, \mathrm{~K}=26, \mathrm{~S}=1$

$\mathrm{R}=22, \mathrm{Q}=9503, \mathrm{H}=1, \mathrm{~L}=2, \mathrm{~K}=42, \mathrm{P}=1$

$\mathrm{R}=10, \mathrm{Q}=9545, \mathrm{H}=8, \mathrm{~L}=4, \mathrm{~K}=2, \mathrm{P}=1, \mathrm{Xa} a=1$

$\mathrm{N}=1166, \mathrm{D}=5, \mathrm{G}=12, \mathrm{I}=1, \mathrm{~K}=1, \mathrm{~S}=8381, \mathrm{~T}=4, \mathrm{Xaa}=1$

$\mathrm{A}=9536, \mathrm{G}=1, \mathrm{~T}=29, \mathrm{~V}=5$

$\mathrm{A}=9, \mathrm{G}=14, \mathrm{~L}=1, \mathrm{M}=4, \mathrm{~W}=1, \mathrm{~V}=9542$

$\mathrm{N}=18, \mathrm{D}=9515, \mathrm{E}=2, \mathrm{G}=36$

$\mathrm{N}=2, \mathrm{D}=9558, \mathrm{E}=4, \mathrm{H}=1, \mathrm{~T}=2, \mathrm{Y}=2, \mathrm{~V}=1, \mathrm{X} a \mathrm{a}=1$

$A=6, R=1, N=196, D=9261, E=4, G=53, H=6, I=1, M=1, T=1, Y=15, V=25, X a$

$\mathrm{R}=1, \mathrm{D}=28, \mathrm{C}=18, \mathrm{G}=9288, \mathrm{~S}=208, \mathrm{~V}=26, \mathrm{X} a \mathrm{a}=2$

$\mathrm{R}=3, \mathrm{~N}=1, \mathrm{Q}=7, \mathrm{H}=9555, \mathrm{P}=3, \mathrm{~T}=1, \mathrm{Y}=1$

$\mathrm{A}=1, \mathrm{~L}=14, \mathrm{~F}=9556$

$\mathrm{A}=60, \mathrm{C}=5, \mathrm{~L}=1, \mathrm{~F}=1, \mathrm{~V}=9504$

$\mathrm{N}=9519, \mathrm{D}=35, \mathrm{H}=8, \mathrm{~S}=6, \mathrm{Y}=3$

$\mathrm{R}=2, \mathrm{G}=1, \mathrm{I}=9549, \mathrm{~L}=1, \mathrm{~K}=1, \mathrm{M}=4, \mathrm{~T}=3, \mathrm{~V}=10$

$\mathrm{A}=5, \mathrm{D}=1, \mathrm{Q}=11, \mathrm{E}=9498, \mathrm{G}=5, \mathrm{~K}=21, \mathrm{~S}=1, \mathrm{~V}=26, \mathrm{Xaa}=3$

$\mathrm{Q}=1, \mathrm{~L}=9515, \mathrm{~K}=3, \mathrm{M}=32, \mathrm{~F}=2, \mathrm{P}=17, \mathrm{Xaa}=1$

$\mathrm{R}=1, \mathrm{D}=6, \mathrm{E}=9358, \mathrm{G}=38, \mathrm{~K}=167, \mathrm{~V}=1$ 


\begin{tabular}{|c|c|}
\hline $\begin{array}{l}= \\
= \\
y\end{array}$ & $=$ \\
\hline 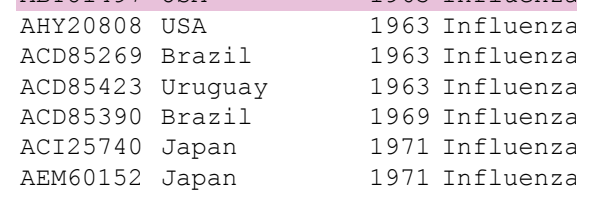 & 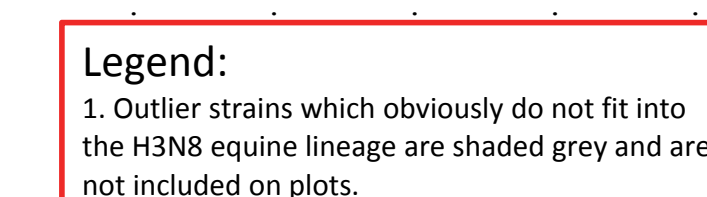 \\
\hline 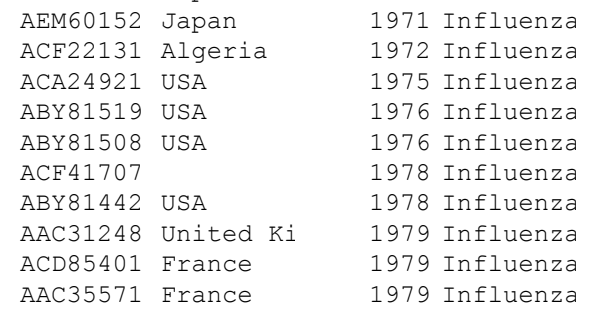 & 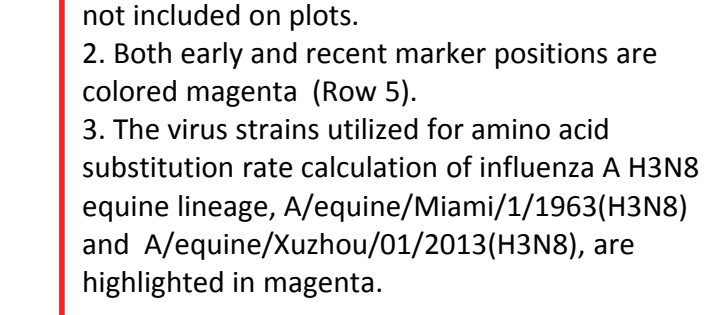 \\
\hline
\end{tabular}





$$
11
$$





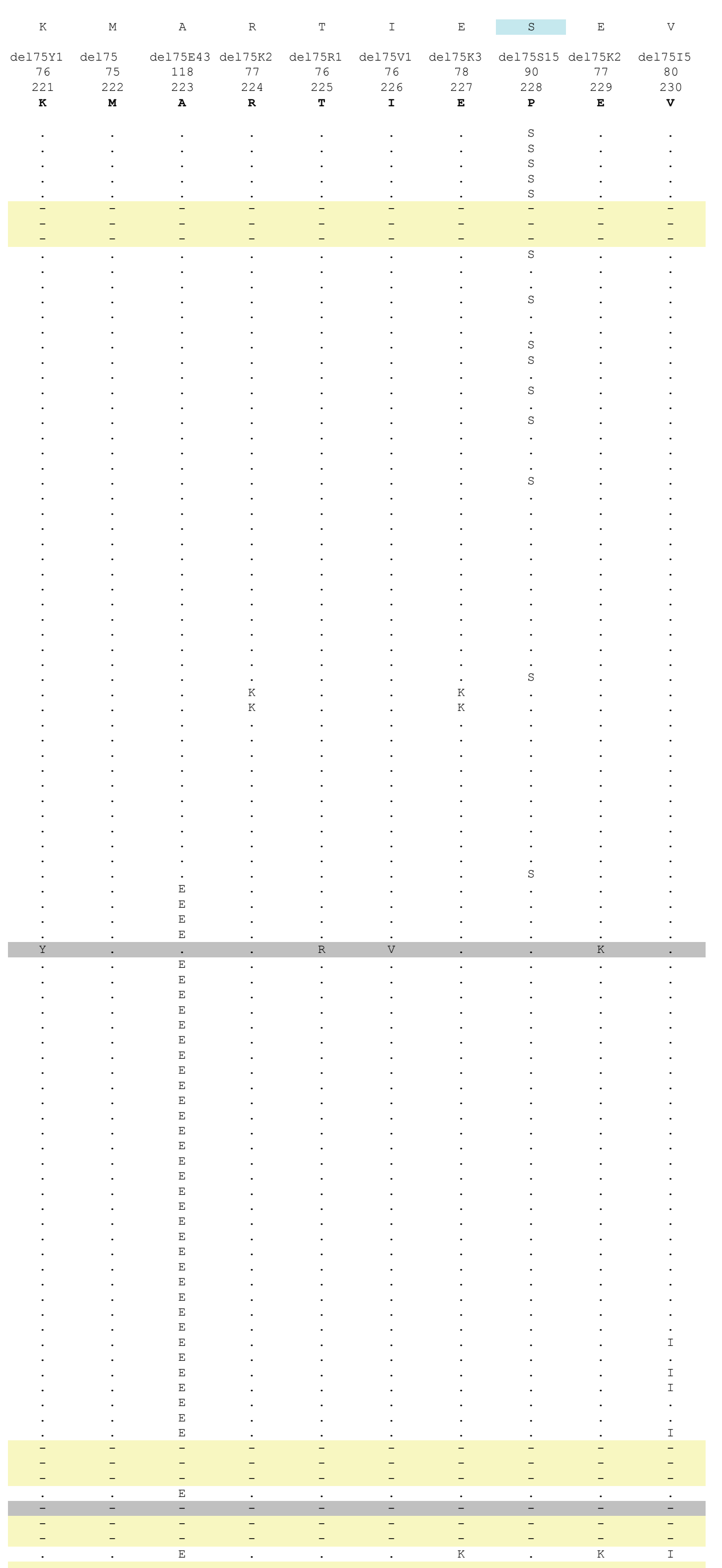




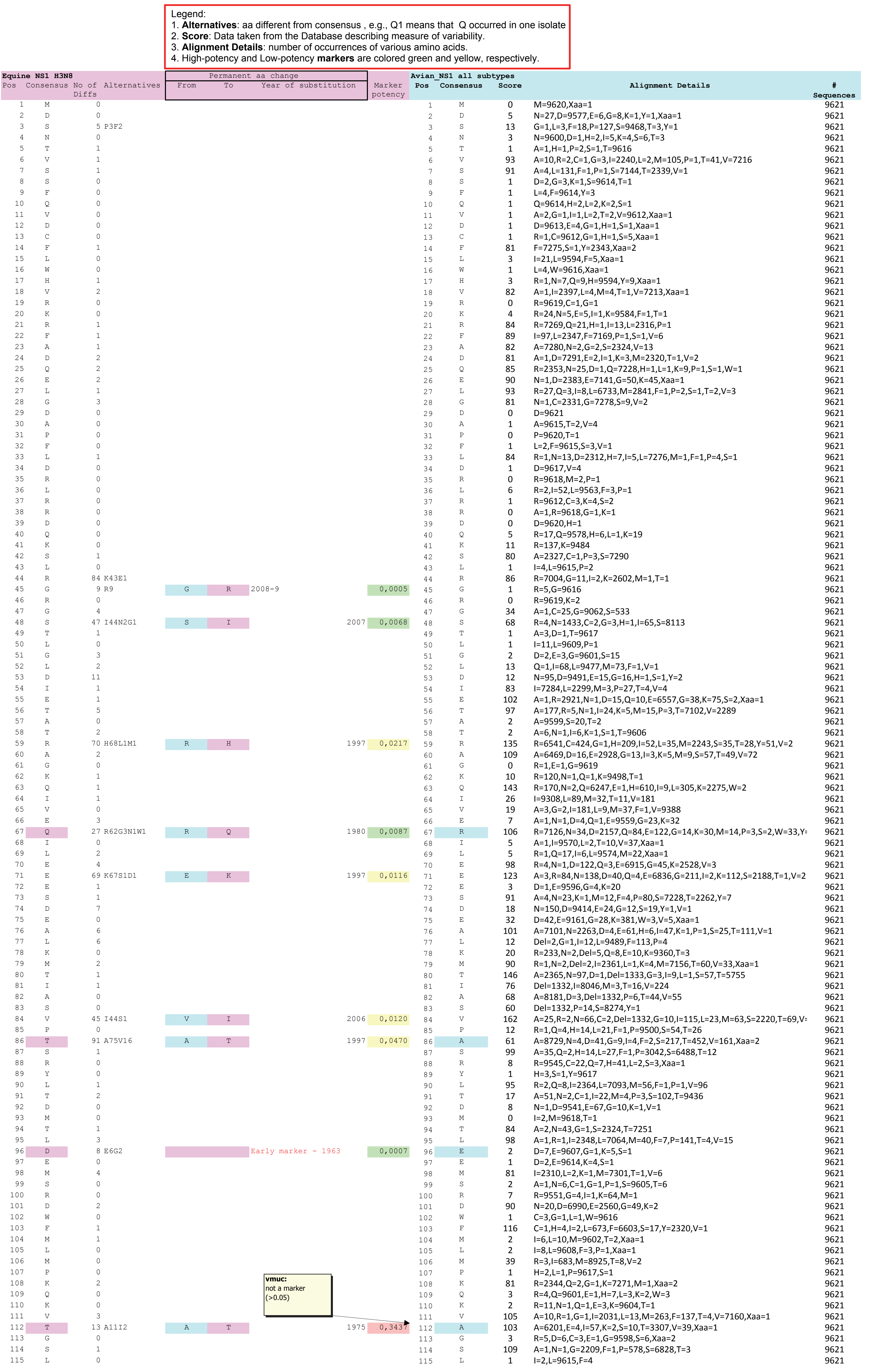




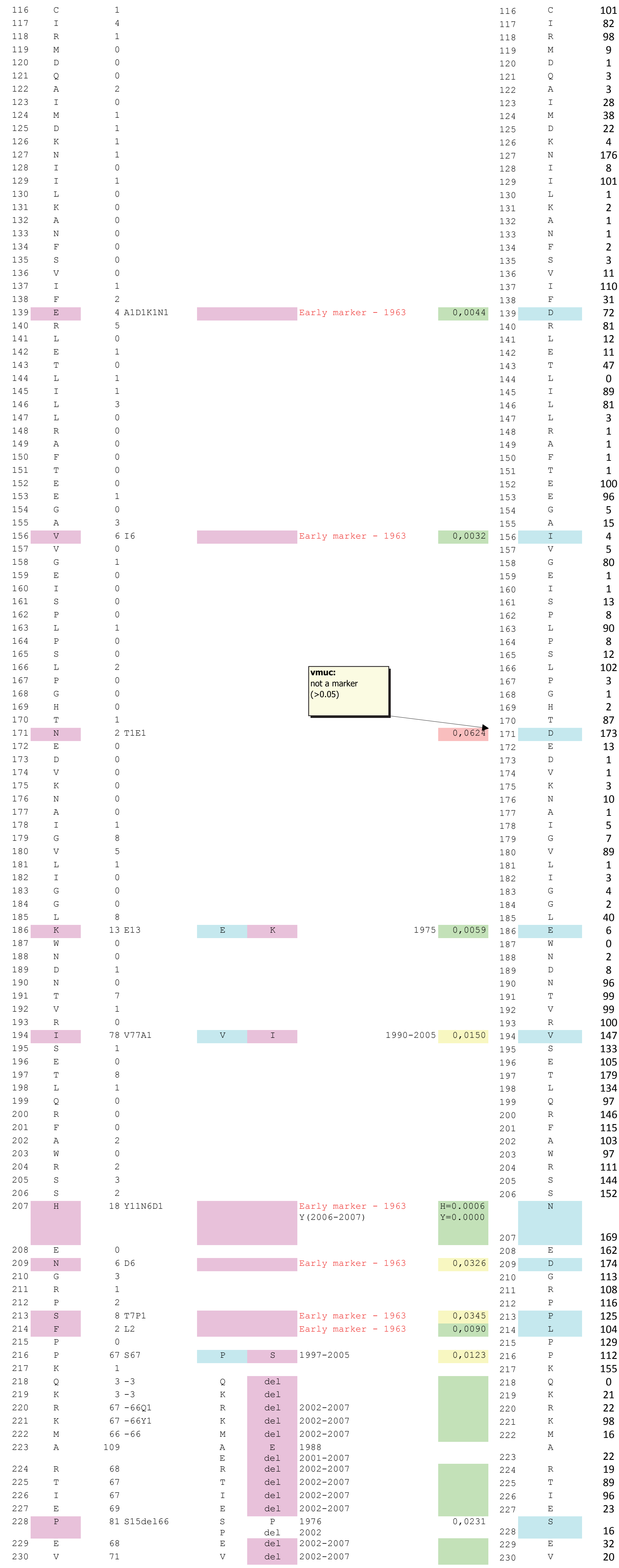

$\mathrm{R}=66, \mathrm{C}=7153, \mathrm{G}=1, \mathrm{l}=99, \mathrm{~L}=6, \mathrm{M}=2211, \mathrm{~F}=6, \mathrm{~S}=47, \mathrm{~W}=2, \mathrm{Y}=16, \mathrm{~V}=14$

$A=1$, Del $=1, I=7250, M=2, F=2, T=4, V=2361$

$\mathrm{R}=5589$, Del $=1, \mathrm{~K}=4030, \mathrm{Xaa}=1$

Del $=3, \mathrm{l}=23, \mathrm{~L}=47, \mathrm{M}=9536, \mathrm{~T}=1, \mathrm{~V}=11$

$\mathrm{D}=9617, \mathrm{Del}=3, \mathrm{~V}=1$

$\mathrm{R}=2, \mathrm{~N}=1, \mathrm{Del}=4, \mathrm{Q}=9594, \mathrm{H}=17, \mathrm{~K}=2, \mathrm{P}=1$

A $=9600, \mathrm{Del}=4, \mathrm{E}=1, \mathrm{~S}=13, \mathrm{~T}=3$

26, T=293, V=54

$A=15, R=2$, Del=4, $=119, L=46, \mathrm{M}=9127, \mathrm{~S}=1, \mathrm{~T}=13, \mathrm{~V}=294$

$\mathrm{N}=221, \mathrm{D}=9343, \mathrm{Del}=4, \mathrm{E}=26, \mathrm{G}=26, \mathrm{~V}=1$

$\mathrm{R}=16, \mathrm{~N}=12, \mathrm{Del}=4, \mathrm{Q}=1, \mathrm{E}=2, \mathrm{~K}=9586$

$\mathrm{A}=12, \mathrm{R}=2261, \mathrm{~N}=5336, \mathrm{D}=90, \mathrm{Del}=4, \mathrm{E}=66, \mathrm{l}=48, \mathrm{~K}=124, \mathrm{M}=1, \mathrm{P}=4, \mathrm{~S}=18, \mathrm{~T}=1$ Del $=4, I=9537, L=3, T=1, V=76$

$\mathrm{R}=1, \mathrm{Del}=4, \mathrm{l}=7255, \mathrm{~L}=24, \mathrm{~K}=32, \mathrm{M}=167, \mathrm{~S}=3, \mathrm{~T}=2046, \mathrm{~V}=88$, $\mathrm{Xaa}=1$

Del=4, I=2,L=9612, $\mathrm{F}=2, \mathrm{P}=1$

$\mathrm{R}=9, \mathrm{~N}=1, \mathrm{Del}=4, \mathrm{E}=1, \mathrm{~K}=9606$

$\mathrm{A}=9614, \mathrm{Del}=4, \mathrm{G}=2, \mathrm{Xaa}=1$

$\mathrm{N}=9612, \mathrm{D}=1$, Del $=4, \mathrm{~K}=1, \mathrm{~S}=1, \mathrm{~T}=1, \mathrm{Xaa}=1$

Del=4,L=11,F=9605, V=1

$\mathrm{R}=2, \mathrm{~N}=12, \mathrm{C}=1, \mathrm{Del}=4, \mathrm{G}=2, \mathrm{~S}=9597, \mathrm{~T}=2$, $\mathrm{Xaa}=1$

$A=13$, Del $=4, l=80, L=7, M=7, V=9509, X a a=1$

$A=34, \mathrm{~N}=4, \mathrm{Del}=4, \mathrm{G}=1, \mathrm{l}=6932, \mathrm{~L}=2314, \mathrm{M}=43, \mathrm{~F}=10, \mathrm{~S}=6, \mathrm{~T}=182, \mathrm{~V}=90, \mathrm{Xaa}=$

$\mathrm{C}=7, \mathrm{Del}=4, \mathrm{~L}=27, \mathrm{~F}=9178, \mathrm{~S}=34, \mathrm{Y}=371$

$\mathrm{N}=1277, \mathrm{D}=8158, \mathrm{Del}=4, \mathrm{E}=42, \mathrm{G}=138, \mathrm{~S}=1, \mathrm{Xaa}=1$

$R=7273$, Del $=3, Q=2339, H=1, K=3, P=1, W=1$

Del=3,l=5,L=9481, M=130, $F=1, S=1$

$\mathrm{D}=99, \mathrm{Del}=3, \mathrm{E}=9504, \mathrm{G}=8, \mathrm{~K}=7$

$A=625, N=124$, Del $=3, l=6, S=11, T=8852$

Del $=3, L=9618$

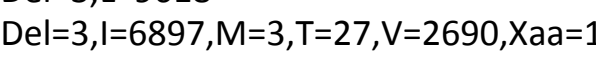

$\mathrm{C}=1, \mathrm{Del}=3, \mathrm{~L}=7292, \mathrm{M}=1, \mathrm{~F}=1, \mathrm{P}=1, \mathrm{~S}=2320, \mathrm{Xaa}=2$

Del=3,H=1,l=19,L=9595, $\mathrm{V}=3$

$\mathrm{R}=9613, \mathrm{Del}=3, \mathrm{G}=2, \mathrm{~K}=3$

$A=9614, D=1$, Del $=3, P=1, T=1$, Xaa $=1$

Del $=3, L=7, F=9611$

Del $=3, S=8, T=9610$

$\mathrm{R}=4, \mathrm{~N}=94, \mathrm{D}=2538$, Del=3, $\mathrm{E}=6897, \mathrm{G}=16, \mathrm{~K}=22, \mathrm{~S}=47$

$\mathrm{N}=26, \mathrm{D}=2373, \mathrm{Del}=3, \mathrm{E}=7063, \mathrm{G}=125, \mathrm{H}=1, \mathrm{~K}=8, \mathrm{~S}=20, \mathrm{Y}=1, \mathrm{Xaa}=1$

$\mathrm{R}=4, \mathrm{~N}=1, \mathrm{D}=11, \mathrm{Del}=3, \mathrm{E}=4, \mathrm{G}=9580, \mathrm{~S}=18$

$\mathrm{A}=9458, \mathrm{D}=1, \mathrm{Del}=3, \mathrm{E}=3, \mathrm{G}=1, \mathrm{~S}=30, \mathrm{~T}=82, \mathrm{~V}=42, \mathrm{Xaa}=1$

Del=3,l=9583,M=1,F=1,S=1,T=1,V=31

$A=10$, Del $=3, l=31, V=9577$

$A=2328$, Del $=3, G=7290$

Del=3,E=9611, G=1, K=2, V=4

Del $=3, I=9611, V=7$

Del $=3, L=5, F=2, P=1, S=9469, T=5, Y=136$

$\mathrm{Q}=76, \mathrm{~L}=2, \mathrm{P}=9538, \mathrm{~S}=4, \mathrm{~T}=1$

$\mathrm{I}=2232, L=7257, \mathrm{M}=5, \mathrm{~F}=94, \mathrm{~S}=3, \mathrm{~V}=30$

$A=1, H=15, L=13, P=9552, S=26, T=3, W=2, Y=8$

$A=1, H=15, L=13, P=9552, S=26$
$F=30, P=1, S=9488, T=2, Y=99$

$R=2, I=24, L=6980, M=2295, F=312, P=2, T=1, V=3, X a a=1$

$A=1, G=2, L=4, P=9599, S=14$

$\mathrm{E}=4, \mathrm{G}=9614$

$\mathrm{R}=2, \mathrm{H}=9605, \mathrm{~L}=2, \mathrm{P}=1, \mathrm{~S}=1, \mathrm{Y}=7$

$A=4, N=49, I=17, F=1, S=2328, T=7219$

$=1, \mathrm{~A}=99, \mathrm{R}=6, \mathrm{~N}=600, \mathrm{D}=5686, \mathrm{E}=113, \mathrm{G}=862, \mathrm{~K}=6, \mathrm{~S}=78, \mathrm{~T}=2146, \mathrm{Y}=19, \mathrm{~V}=2$

$\mathrm{Q}=1, \mathrm{E}=9455, \mathrm{G}=1, \mathrm{~K}=161$

$\mathrm{N}=2, \mathrm{D}=9614, \mathrm{E}=1, \mathrm{G}=1$

$\mathrm{R}=1, \mathrm{~N}=1, \mathrm{Q}=20, \mathrm{E}=2, \mathrm{~K}=959$

$\mathrm{N}=9520, \mathrm{D}=50, \mathrm{G}=1, \mathrm{H}=3, \mathrm{l}=25, \mathrm{~K}=1, \mathrm{M}=1, \mathrm{~S}=15, \mathrm{~T}=2$

$A=9611, C=1, G=1, S=3, T=2$

$\mathrm{N}=2, \mathrm{I}=9573, \mathrm{~L}=1, \mathrm{M}=3, \mathrm{~F}=1, \mathrm{~W}=1, \mathrm{~V}=37$

$\mathrm{R}=5, \mathrm{D}=11, \mathrm{E}=55, \mathrm{G}=9545, \mathrm{P}=1, \mathrm{~S}=1$

$A=23, D=6, G=3, l=2637, M=1, S=1, T=7, V=6940$

$\mathrm{R}=1, \mathrm{I}=2, \mathrm{~L}=9610, \mathrm{~F}=3, \mathrm{P}=1, \mathrm{~S}=1$

$\mathrm{I}=9589, \mathrm{~L}=2, \mathrm{M}=1, \mathrm{~S}=1, \mathrm{~T}=9, \mathrm{~V}=16$

$\mathrm{R}=21, \mathrm{D}=6, \mathrm{G}=9586, \mathrm{~K}=1, \mathrm{~S}=3, \mathrm{Xaa}=1$

$\mathrm{R}=2, \mathrm{D}=1, \mathrm{E}=8, \mathrm{G}=9606, \mathrm{~S}=1$

$L=8872, F=744, P=1, T=1$

$\mathrm{D}=1, \mathrm{E}=9557, \mathrm{G}=2, \mathrm{~K}=57, \mathrm{~V}=1$

$\mathrm{R}=1, \mathrm{C}=1, \mathrm{~W}=9615, \mathrm{Xaa}=1$

$\mathrm{R}=1, \mathrm{~N}=9604, \mathrm{~K}=1, \mathrm{~S}=12$

$\mathrm{N}=34, \mathrm{D}=9536, \mathrm{E}=1, \mathrm{G}=42, \mathrm{Y}=5$

$N=5983, D=1, \mathrm{Del}=3633, Y=1$

$\mathrm{A}=4, \mathrm{Del}=3633, \mathrm{P}=1, \mathrm{~S}=26$
$\mathrm{Del}=3633, \mathrm{l}=40, \mathrm{~V}=5945$

Del $=3633, I=40, V=5945$

$\mathrm{R}=5939, \mathrm{~N}=3628, \mathrm{Q}=47, \mathrm{H}=1, \mathrm{Y}=3$

$A=31, G=12, I=144, L=5, K=1, F=1, P=4, S=2279, T=1343, V=5798$

$\mathrm{I}=2299, \mathrm{~L}=2, \mathrm{~F}=1, \mathrm{P}=1, \mathrm{~S}=5977, \mathrm{~T}=5, \mathrm{~V}=1331, \mathrm{Xaa}=2$

$\mathrm{R}=3516, \mathrm{D}=1, \mathrm{Q}=118, \mathrm{E}=5974, \mathrm{~K}=7, \mathrm{P}=1, \mathrm{Xaa}=1$

$A=2429, N=485, D=3, I=435, K=1, S=4, T=5091, V=1170$

$\mathrm{I}=347, \mathrm{~L}=5629, \mathrm{~F}=2, \mathrm{~S}=3282, \mathrm{~T}=348, \mathrm{~V}=8, \mathrm{Xaa}=2$

$\mathrm{Q}=5984, \mathrm{E}=3623, \mathrm{G}=2, \mathrm{H}=1, \mathrm{~K}=8$

$A=103, R=5974, N=2358, D=1, E=1, I=161, K=9, S=10, T=1001$

$\mathrm{I}=3248, \mathrm{~L}=384, \mathrm{~F}=5978, \mathrm{Y}=6, \mathrm{~V}=2$

$A=5901, R=1, D=2, Q=3630, H=2, I=1, P=1, S=8, T=72$

$\mathrm{R}=3629, \mathrm{~K}=7, \mathrm{~S}=1, \mathrm{~W}=5977, \mathrm{Xaa}=1$

$\mathrm{R}=5893, \mathrm{C}=1, \mathrm{Del}=2, \mathrm{G}=43, \mathrm{~L}=4, \mathrm{~K}=44, \mathrm{~F}=3527, \mathrm{Y}=99, \mathrm{~V}=2$

$A=3512, R=8, N=294, C=1, G=275, l=41, K=4, F=1, S=5353, T=108, V=14, X a a=$

$A=3512, R=8, N=294, C=1, G=275, I=41, K=4, F=1, S=5353, T=108, V=14, X a a=$
$R=63, N=30, C=613, E=1, G=24, H=1, I=201, L=1, F=2, S=5020, W=3629, V=29$

$R=1109, N=5049, D=849, E=3, G=2586, H=6, K=9, S=3, W=1$

9615

$A=4, N=14, E=5975, G=92, I=813, L=3, K=6, S=1231, T=2, V=1474, X a a=1 \quad 9615$ $=1, \mathrm{R}=2214, \mathrm{~N}=313, \mathrm{D}=5557, \mathrm{C}=52, \mathrm{G}=126, \mathrm{H}=26, \mathrm{I}=137, \mathrm{~L}=1, \mathrm{~S}=1156, \mathrm{~T}=1, \mathrm{~V}=9615$ $\mathrm{R}=26, \mathrm{~N}=229, \mathrm{D}=3387, \mathrm{E}=2, \mathrm{G}=5956, \mathrm{H}=4, \mathrm{~W}=8, \mathrm{Y}=3$

$\mathrm{R}=5807, \mathrm{Del}=3632, \mathrm{G}=176$

$\mathrm{A}=1, \mathrm{Del}=3632, \mathrm{H}=2, \mathrm{~L}=293, \mathrm{P}=5659, \mathrm{~S}=26, \mathrm{~T}=2$

Del $=3632, \mathrm{~L}=108, \mathrm{~K}=1, \mathrm{P}=5521, \mathrm{~S}=332, \mathrm{~T}=21$

$\mathrm{R}=1, \mathrm{Del}=3632, \mathrm{H}=6, \mathrm{l}=1, \mathrm{~L}=5887, \mathrm{~F}=87, \mathrm{P}=1$

$\mathrm{Del}=3632, L=51, P=5343, S=584, T=2, Y=3$

$A=21, E=3603, K=29, P=5813, S=118, T=30, V=1$

$\mathrm{R}=5, \mathrm{~N}=2397, \mathrm{D}=1377, \mathrm{E}=39, \mathrm{G}=97, \mathrm{~K}=5598, \mathrm{~S}=54, \mathrm{~T}=14, \mathrm{~V}=31, \mathrm{Xaa}=3$

$\mathrm{Q}=8694, \mathrm{~S}=1, \mathrm{Xaa}=1$

$\mathrm{A}=1, \mathrm{~N}=97, \mathrm{E}=138, \mathrm{~K}=8460$

$\mathrm{R}=8437, \mathrm{C}=57, \mathrm{Q}=107, \mathrm{~L}=2, \mathrm{P}=4, \mathrm{~W}=56$

$\mathrm{R}=5, \mathrm{~N}=5, \mathrm{D}=1, \mathrm{C}=33, \mathrm{Q}=1, \mathrm{E}=57, \mathrm{H}=45, \mathrm{~K}=6269, \mathrm{~F}=3, \mathrm{~T}=3, \mathrm{Y}=2211$

$\mathrm{A}=8338, \mathrm{E}=293, \mathrm{~L}=1, \mathrm{~S}=1$

$\mathrm{R}=8421, \mathrm{E}=1, \mathrm{G}=178, \mathrm{l}=3, \mathrm{~L}=1, \mathrm{~K}=26, \mathrm{~W}=3$

$A=33, R=2223, E=4, G=15, K=2, P=1, S=2, T=6299$

$A=59, I=6255, S=1, T=94, V=2170$

$R=1, E=8314, G=164, K=100$

$Q=1, P=198, S=8379, T=1$

$\mathrm{A}=1, \mathrm{E}=8042, \mathrm{~K}=487$

$A=1, I=256, F=6, V=8267$ 

Legend:

Alternatives: aa different from consensus, e.g., Q1 means that $Q$ occurred in one isolate . Score: Data taken from the Database describing measure of variability.

3. Alignment Details: number of occurrences of various amino acids.

4. High-potency and Low-potency markers are colored green and yellow, respectively.
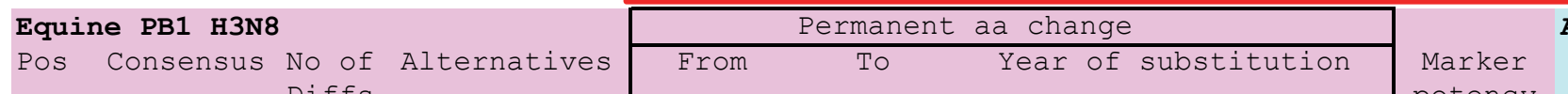

P3F2

$\mathrm{T} 1$

K101

K2R2E1

G3V

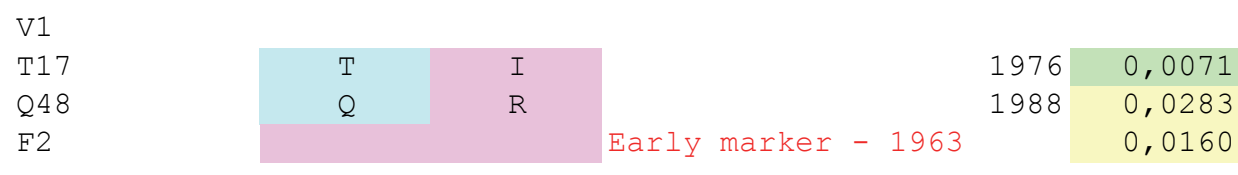

S1T1

I3

Y1

N2

Pos Consensus Scor

$5 \mathrm{~N}=24, \mathrm{D}=8965, \mathrm{E}=6, \mathrm{G}=8, \mathrm{~K}=1, \mathrm{Y}=2, \mathrm{Xaa}=1$

$13 \mathrm{G}=1, \mathrm{~L}=3, \mathrm{~F}=16, \operatorname{PrO}=123, \mathrm{~S}=8860, \mathrm{~T}=3, \mathrm{Y}=1$
$3 \mathrm{~N}=8985, \mathrm{D}=1, \mathrm{H}=2, \mathrm{I}=5, \mathrm{~K}=4, \mathrm{~F}=1, \mathrm{~S}=6, \mathrm{~T}=3$

$0 \mathrm{H}=1, \operatorname{Pro}=1, \mathrm{~S}=1, \mathrm{~T}=9004$

$93 \mathrm{~A}=8, \mathrm{R}=2, \mathrm{Cys}=1, \mathrm{G}=3, \mathrm{I}=2087, \mathrm{~L}=2, \mathrm{M}=106, \mathrm{~T}=38, \mathrm{~V}=6760$

$91 \mathrm{~A}=4, \mathrm{~L}=127, \mathrm{~F}=1, \operatorname{Pro}=1, \mathrm{~S}=6689, \mathrm{~T}=2184, \mathrm{~V}=1$

$1 \mathrm{D}=2, \mathrm{G}=3, \mathrm{~K}=1, \mathrm{~S}=8999, \mathrm{~T}=2$

$1 \mathrm{Del}=1, \mathrm{~L}=4, \mathrm{~F}=8999, \mathrm{Y}=3$

$1 \mathrm{Del}=4, \mathrm{Q}=8997, \mathrm{H}=2, \mathrm{~L}=2, \mathrm{~K}=1, \mathrm{~S}=1$

$8 \mathrm{~A}=4, \mathrm{~N}=4, \mathrm{D}=8929, \mathrm{De}=1, \mathrm{G}=70$

$3 \mathrm{I}=8988, \mathrm{M}=4, \mathrm{~F}=1, \mathrm{D}$
$5 \mathrm{~L}=8957, \mathrm{M}=46, \mathrm{~V}=5$

$163 \mathrm{~A}=13, \mathrm{R}=14, \mathrm{Q}=2073, \mathrm{E}=91, \mathrm{G}=1, \mathrm{I}=77, \mathrm{~L}=21, \mathrm{~K}=91, \mathrm{M}=5541, \mathrm{~T}=272, \mathrm{~V}=81$

$\begin{array}{ll}1 & I=2, M=900, \\ 0 & S=9008\end{array}$

$2 \mathrm{R}=7, \mathrm{~N}=3, \mathrm{~K}=8997, \mathrm{~T}=1$

$5 \mathrm{~N}=1, \mathrm{I}=4, \mathrm{~L}=25, \mathrm{M}=8963, \mathrm{~V}=15$

$19 \mathrm{~A}=1, \mathrm{R}=246, \mathrm{Q}=8758, \mathrm{E}=1, \mathrm{H}=1, \mathrm{PrO}=1$

$1 \mathrm{I}=1, \mathrm{~L}=9000, \mathrm{M}=2, \mathrm{~S}=2, \mathrm{~W}=3$

$138 \mathrm{~A}=1137, \mathrm{R}=49, \mathrm{E}=2345, \mathrm{G}=5459, \mathrm{~K}=2, \mathrm{~T}=11, \mathrm{~V}=5$

$14 \mathrm{~A}=3, \mathrm{~F}=11, \operatorname{Pro}=28, \mathrm{~S}=8873, \mathrm{~T}=6, \mathrm{Y}=86, \mathrm{~V}=1$

$2 \mathrm{H}=1, \mathrm{~L}=14, \mathrm{~F}=1, \operatorname{PrO}=1, \mathrm{~S}=8991$

$97 \mathrm{~A}=24, \mathrm{E}=6639, \mathrm{G}=190, \mathrm{~K}=1, \mathrm{M}=4, \mathrm{~V}=2149, \mathrm{Xaa}=$

$9 \mathrm{~N}=8, \mathrm{D}=8912, \mathrm{G}=87, \mathrm{~V}=1$

$1 \mathrm{~L}=9002, \mathrm{~S}=2, \mathrm{~W}=4$

$12 \mathrm{~N}=8871, \mathrm{D}=2, \mathrm{~K}=1, \mathrm{~S}=131, \mathrm{~T}=1, \mathrm{Y}=1, \mathrm{Xaa}=1$

O $\mathrm{G}=9007, \mathrm{~V}=1$

$5 \mathrm{I}=36, \mathrm{~K}=1, \mathrm{M}=8961, \mathrm{~V}=10$

$4 \mathrm{I}=8967, \mathrm{M}=1, \mathrm{~T}=1, \mathrm{~V}=39$

$6 \mathrm{I}=64, \mathrm{~T}=8944$

$19 \mathrm{R}=255, \mathrm{Q}=8747, \mathrm{H}=2, \mathrm{~L}=3, \mathrm{PrO}=1$

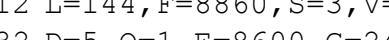

, K=152, Xaa=

$3 \mathrm{~A}=11, \mathrm{R}=2159, \mathrm{Cys}=8, \mathrm{G}=1, \mathrm{H}=4, \mathrm{~F}=3, \mathrm{~S}=6820, \mathrm{~T}=2$

$\mathrm{E}=2, \mathrm{I}=1, \mathrm{~K}=8775, \mathrm{Xaa}=1$

$94 \mathrm{R}=1, \mathrm{I}=2632, \mathrm{~L}=6311, \mathrm{~K}=1, \mathrm{M}=2, \mathrm{~F}=34, \mathrm{~T}=18, \mathrm{~V}=9$

$0 \mathrm{~L}=1, \mathrm{~F}=1, \mathrm{Y}=9005, \mathrm{Xa} a=1$

$3=1, A=1, N=14, D=8984, E=2, G=3, V=3$

$23 \mathrm{~A}=1, \mathrm{~L}=179, \mathrm{M}=1, \mathrm{~S}=8726, \mathrm{~T}=101$

$2 \mathrm{I}=3, \mathrm{~L}=8994, \mathrm{~F}=11$

$1 \mathrm{E}=5, \mathrm{G}=9003$

政

108 $\mathrm{A}=6453, \mathrm{R}=1, \mathrm{~N}=1, \mathrm{D}=7, \mathrm{E}=1, \mathrm{I}=5, \mathrm{~K}=3, \mathrm{~F}=2, \mathrm{PrO}=5, \mathrm{~S}=2182, \mathrm{~T}=285, \mathrm{~V}=59$ $62 \mathrm{~A}=502, \mathrm{I}=31, \mathrm{~L}=353, \mathrm{M}=29, \mathrm{~S}$

$10 \mathrm{R}=1, \mathrm{I}=33, \mathrm{M}=8902, \mathrm{~V}=72$

$45 \mathrm{I}=276, \mathrm{~L}=54, \mathrm{M}=8415, \mathrm{~T}=17, \mathrm{~V}=244, \mathrm{Xaa}=2$

$2 \mathrm{R}=19, \mathrm{G}=8989$

$1 \mathrm{~N}=1, \mathrm{D}=8998, \mathrm{E}=6, \mathrm{G}=2, \mathrm{~V}=1$

$58 \mathrm{I}=6, \mathrm{~L}=7787, \mathrm{~F}=1212$, $\operatorname{Pr} \mathrm{O}=1$

$1 \mathrm{Q}=1, \mathrm{H}=8881, \mathrm{~L}=4, \mathrm{Y}=122$

$17 \mathrm{~A}=1, \mathrm{~L}=4, \mathrm{H}=1, \mathrm{~L}=31, \mathrm{P}=99, \mathrm{PrO}=2, \mathrm{~S}=8838, \mathrm{~T}=6, \mathrm{Y}=26$

1. $1=3, L=8845, \mathrm{P}=157, \mathrm{Pro}=1, \mathrm{~S}=1, \mathrm{~V}=1$

$132 \mathrm{~A}=3, \mathrm{R}=11, \mathrm{~N}=1444, \mathrm{D}=2, \mathrm{G}=3, \mathrm{H}=1, \mathrm{I}=1109, \mathrm{~S}=6231, \mathrm{~T}=198, \mathrm{~V}=5$, $\mathrm{Xaa}=1$ $8 \mathrm{R}=8918, \mathrm{~K}=90$

$100 \mathrm{~A}=2170, \mathrm{R}=30, \mathrm{E}=92, \mathrm{G}=6623, \mathrm{~K}=50, \mathrm{~S}=1, \mathrm{~W}=23, \mathrm{~V}=19$

$97 \mathrm{~A}=34, \mathrm{R}=14, \mathrm{~N}=73, \mathrm{D}=10, \mathrm{Q}=7, \mathrm{E}=10, \mathrm{I}=9, \mathrm{~K}=6724, \mathrm{~S}=22, \mathrm{~T}=2104, \mathrm{Xaa}=1$

0 CYs $=3, \mathrm{~W}=9005$

$0 \mathrm{R}=9007, \mathrm{~K}=1$

$39 \mathrm{R}=23, \mathrm{~N}=16, \mathrm{D}=496, \mathrm{Q}=1, \mathrm{E}=8440, \mathrm{G}=14, \mathrm{~K}=15, \mathrm{Y}=3$

$83 \mathrm{R}=5, \mathrm{Q}=6824, \mathrm{E}=2151, \mathrm{G}=16, \mathrm{H}=1, \mathrm{~L}=1, \mathrm{~K}=10$

$0 \mathrm{~L}=9006, \mathrm{M}=1, \mathrm{~V}=1$

$29 \mathrm{R}=1, \mathrm{~N}=1, \mathrm{D}=1, \mathrm{G}=440, \mathrm{~S}=8563$, $\mathrm{Xaa}=1$

$8 \mathrm{R}=24, \mathrm{Q}=8937, \mathrm{H}=42, \mathrm{~L}=3, \mathrm{~K}=1$

2 $\mathrm{R}=8, \mathrm{~N}=5, \mathrm{Q}=1, \mathrm{~K}=8993$

$0 \mathrm{~L}=1, \mathrm{~F}=9005, \mathrm{~S}=1$

$2 \mathrm{D}=7, \mathrm{E}=8989, \mathrm{G}=10, \mathrm{~V}=$

O $\mathrm{E}=9004, \mathrm{~K}=3$

$8 \mathrm{I}=8938, \mathrm{~L}=4, \mathrm{~K}=1, \mathrm{M}=41, \mathrm{~T}=2, \mathrm{~V}=21$

$21 \mathrm{R}=8712, \mathrm{~K}=294, \mathrm{~T}=1$

$1 \mathrm{G}=2, \mathrm{~L}=3, \mathrm{~W}=9002$
$5 \mathrm{~L}=8956, \mathrm{M}=48, \mathrm{~S}=1, \mathrm{~V}=2$

$5 \mathrm{~L}=8956, \mathrm{M}=48, \mathrm{~S}=1$,
$4 \mathrm{I}=8967, \mathrm{M}=1, \mathrm{~V}=39$

$4 \mathrm{I}=8967, \mathrm{M}=1, \mathrm{~V}=39$

87 $\mathrm{A}=2163, \mathrm{D}=4, \mathrm{E}=6768, \mathrm{G}=66, \mathrm{~S}=$

(1) $A=2, R=2, C=8993,68, I=371, L$

$0 \mathrm{R}=9006, \mathrm{Q}=1$

作

$96 \mathrm{R}=6671, \mathrm{G}=2, \mathrm{I}=2136, \mathrm{~K}=157, \mathrm{M}=7, \mathrm{~S}=1, \mathrm{~T}=3, \mathrm{~V}=30$

$1 \mathrm{~L}=9003, \mathrm{~F}=1, \mathrm{~W}=3$

$98 \mathrm{~A}=36, \mathrm{R}=173, \mathrm{~N}=13, \mathrm{Q}=1, \mathrm{I}=6, \mathrm{~K}=6659, \mathrm{M}=3, \mathrm{~S}=1, \mathrm{~T}=2115$

$49 \mathrm{~A}=16, \mathrm{R}=37, \mathrm{~N}=75, \mathrm{Q}=14, \mathrm{I}=5715, \mathrm{~L}=1, \mathrm{~K}=2115, \mathrm{M}=15, \mathrm{~F}=17, \mathrm{~S}=22, \mathrm{~T}=123$

, $\operatorname{PrO}=2, \mathrm{~S}=2, \mathrm{~T}=8997$

$\mathrm{D}=1, \mathrm{Q}=2, \mathrm{E}=8999, \mathrm{G}=5$

$9 \mathrm{~N}=8915, \mathrm{D}=5, \mathrm{~K}=1, \mathrm{~S}=85, \mathrm{~T}=1$

$2 \mathrm{R}=3, \mathrm{G}=15, \mathrm{~S}=8989$

$4 \mathrm{~L}=1, \mathrm{~F}=8973, \mathrm{~S}=1, \mathrm{Y}=32$

$1 \mathrm{D}=4, \mathrm{Q}=3, \mathrm{E}=8997, \mathrm{G}=2, \mathrm{~K}=1$

$1 \mathrm{R}=3, \mathrm{Q}=9001, \mathrm{H}=3$

$\mathrm{O} N=2, \mathrm{I}=9004, \mathrm{~T}=1$

$0 \mathrm{R}=1, \operatorname{PrO}=2, \mathrm{~T}=9004$

B8 $\mathrm{I}=45, \mathrm{~L}=2202, \mathrm{~K}=1, \mathrm{M}=6733, \mathrm{~T}=1, \mathrm{~V}=24, \mathrm{Xaa}=1$

$2 \mathrm{R}=2, \mathrm{Q}=8996, \mathrm{~L}=4, \mathrm{Pro}=5$

$1 \mathrm{~A}=9001, \mathrm{~S}=1, \mathrm{~T}=5$

$2 \mathrm{Q}=8992, \mathrm{H}=10, \mathrm{~K}=4, \mathrm{P}$

$2 \mathrm{Q}=8992, \mathrm{H}=10, \mathrm{~K}=4, \mathrm{P}$
$2 \mathrm{I}=13, \mathrm{~L}=8993, \mathrm{~V}=1$

$1 \mathrm{~L}=9001, \mathrm{~F}=1, \operatorname{Pro}=1, \mathrm{~W}=1, \mathrm{Y}=1, \mathrm{~V}=1, \mathrm{Xaa}=1$

$1 \mathrm{Q}=1, \mathrm{~L}=9003, \mathrm{~F}=1, \mathrm{PrO}=2$

$2 \mathrm{~A}=5, \mathrm{E}=2, \mathrm{G}=2, \mathrm{I}=2, \mathrm{~K}=1, \mathrm{~L}$

$1 \mathrm{D}=1, \mathrm{E}=9000, \mathrm{G}=4, \mathrm{~K}=2$

$86 \mathrm{R}=1, \mathrm{~N}=59, \mathrm{Q}=6823, \mathrm{G}=4, \mathrm{H}=1, \mathrm{~L}=5, \mathrm{~K}=3, \mathrm{Pro}=3, \mathrm{~S}=2107, \mathrm{Xaa}=1$

$2 \mathrm{~N}=1, \mathrm{D}=12, \mathrm{E}=8993, \mathrm{G}=1$

$7 \mathrm{I}=8934, \mathrm{M}=72, \mathrm{Xaa}=1$

2 $\mathrm{R}=8992, \mathrm{E}=1, \mathrm{G}=1, \mathrm{~K}=7, \mathrm{~S}=2, \mathrm{~T}=1, \mathrm{~W}=1, \mathrm{Xaa}=2$

$41 \mathrm{~A}=700, \mathrm{~N}=1, \mathrm{D}=2, \mathrm{~L}=4, \operatorname{Pro}=2, \mathrm{~S}=1, \mathrm{~T}=8297$

$1 \mathrm{~N}=1, \mathrm{I}=1, \mathrm{~L}=2, \mathrm{~F}=8999, \mathrm{~S}=2, \mathrm{Y}=1, \mathrm{~V}=1$

$1 \mathrm{~A}=1, \mathrm{~S}=9003, \mathrm{~T}=3$

$1 \mathrm{~L}=4, \mathrm{~F}=8998, \mathrm{Y}=2, \mathrm{~V}=3$

$2 \mathrm{R}=3, \mathrm{Q}=8995, \mathrm{H}=4, \mathrm{~L}=1, \mathrm{Pro}=1, \mathrm{~S}=2, \mathrm{Xaa}=1$

$2 \mathrm{R}=2, \mathrm{H}=2, \mathrm{I}=1, \mathrm{~L}=8990, \mathrm{~F}=7$, $\mathrm{PrO}=1, \mathrm{~S}=1, \mathrm{Xaa}=$

$1 \mathrm{I}=8998, \mathrm{~L}=2, \mathrm{M}=2, \mathrm{~F}=2, \mathrm{~S}=1, \mathrm{~T}=1, \mathrm{Y}=1$ 